\title{
PREPARATION AND CHARACTERIZATION OF ELECTROSPUN POLY(D,L-LACTIDE-CO-GLYCOLIDE) SCAFFOLDS FOR VASCULAR TISSUE ENGINEERING AND THE ADVANCEMENT OF AN IN VITRO BLOOD VESSEL MIMIC
}

\author{
A Thesis Presented to the Faculty of \\ California Polytechnic State University, San Luis Obispo
}

In Partial Fulfillment of the Requirements for the Degree

Master of Science in General Engineering with Specialization in Biomedical Engineering

By

Tiffany Richelle Peña

June 2009 
(C) 2009

Tiffany R. Peña

ALL RIGHTS RESERVED 
TITLE:

AUTHOR:

DATE SUBMITTED:

COMMITTEE CHAIR:

COMMITTEE MEMBER:

COMMITTEE MEMBER:
Preparation and Characterization of Electrospun Poly(D,L-lactide-co-glycolide) Scaffolds for Vascular Tissue Engineering and the Advancement of an In Vitro Blood Vessel Mimic

Tiffany Richelle Peña

June 2009

Kristen O'Halloran Cardinal, $\mathrm{PhD}$

Richard Savage, $\mathrm{PhD}$

Robert Crockett, $\mathrm{PhD}$ 


\section{Abstract}

\section{PREPARATION AND CHARACTERIZATION OF ELECTROSPUN POLY(D,L-LACTIDE- CO-GLYCOLIDE) SCAFFFOLDS FOR VASCULAR TISSUE ENGINEERING AND THE ADVANCEMENT OF AN IN VITRO BLOOD VESSEL MIMIC}

Tiffany Richelle Peña

Currently, an estimated 1 in every 3 adult Americans are affected by one or more cardiovascular complications. The most common complication is coronary artery disease, specifically atherosclerosis. Outcomes of balloon angioplasty treatments have been significantly improved with the addition of drug eluting stents to the process. Although both bare metal and drug eluting stents have greatly increased the effectiveness of angioplasty and decreased the occurrence of restenosis, several complications still exist. For this reason, the stent industry is continually advancing toward better stent and drug-eluting designs, deployment methods, and adjuvant drug therapies, necessitating fast, reliable pre-clinical test methods. Recently, advancements in tissue engineering have led to the development of an in vitro blood vessel mimic (BVM) and the feasibility of evaluating cellular response to intravascular device implantation has been demonstrated. There are several physiological and scalability limitations of the current BVM model that must be addressed before effective use of the model can be initiated. The limiting aspect addressed in this thesis is the use of expanded poly(tetrafluorethylene) [ePTFE] scaffolding for the development of the BVM. There are several disadvantages and limitations to ePTFE including high cost and non-native mechanical properties. The ability to produce and tailor scaffolds in-house would greatly impact the scalability, cost effectiveness, and control over scaffold properties for BVM optimization. Also, 
in-house fabrication will open up further avenues of research into optimum scaffold design for better cellular responses when cultured in vitro.

Electrospinning is a relatively simple and economical method of creating tissue engineering constructs with micro-architecture similar to the native extracellular matrix. Based on the clinical problem and the potential for the BVM, the aim of this thesis is to employ electrospinning for the development of poly(D,L-lactide-co-glycolide) [PLGA] vascular scaffolds as a replacement to ePTFE for the BVM. After primary literature review, PLGA was determined an advantageous polymer for tissue engineering vascular scaffolds and electrospinning based on evidence of adequate endothelial cell attachment, mechanical properties similar to the native vessels, controlled degradation, and good biocompatibility. The first phase of this thesis was to develop an acceptable protocol for the fabrication of electrospun PLGA scaffolds by varying solution concentration, flow rate and applied voltage. Electrospun solutions of $15 \mathrm{wt} \%$ PLGA in $\mathrm{CHCl}_{3}$ resulted in continuous un-beaded fibers of 5-6 microns and tensile properties (3-5 MPa) similar to the native vessel. The optimum protocol for electrospinning 15 wt\% PLGA incorporated a flow rate of $5.5 \mathrm{ml} / \mathrm{hr}$ and an applied voltage of $12,000 \mathrm{~V}$. In the second phase of this thesis, final protocol PLGA scaffolds were cultured in vitro with human umbilical vein endothelial cells (HUVECs) up to 6 days. Fluorescent microscopy and SEM analysis suggest the porous nature of the scaffolds was conducive to sub-luminal cellular penetration. Although results were not optimal for developing an endothelium for the ideal BVM design, the potential of using electrospinning for in-house production of scaffolds for tissue engineering was established. Further optimization of the electrospinning protocol to develop nano-sized structural features could enhance the ability to form an intimal lining of endothelial cells for the next generation BVM design. 


\section{Acknowledgements}

I would first like to thank Dr. Kristen O’Halloran Cardinal for her continuous support and encouragement; without her leadership and dedication to the field of tissue engineering, this thesis would not be possible. It has been a privilege working with you and getting to know you.

Additionally, I would like to thank the members of my thesis committee, Dr. Richard Savage and Dr. Robert Crockett. Thank you for your support and cooperation through this process.

I would like to thank my fellow lab members in the Tissue Engineering Lab, Colby James, Marc Dawson, Aubrey Smith and the rest of the gang; it was a pleasure working with all of you. I would especially like to thank Colby for the hours of conversations on electrospinning and for guiding me through the beginning stages of my thesis. Thank you Aubrey, for introducing me to all the joys of histology. I also want to give a big thank you to Tim Melin, for all the training in the machine shop; it would not have been possible without you.

My biggest thanks and love go out to my parents, Edwin and Karen, whose love and direction have always been there for me. I am so blessed to have parents like you. To my sister Natalie, you are my best friend and the one I look up to. To my husband Zach, thank you for being my biggest fan, for supporting and loving me through this long and trying process. 


\section{Table of Contents}

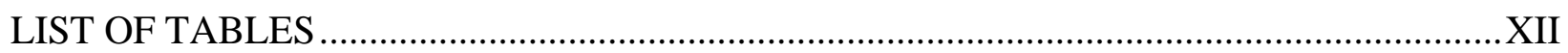

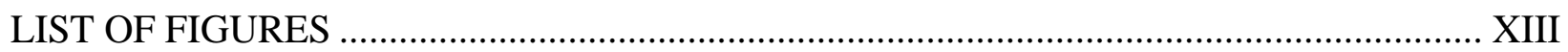

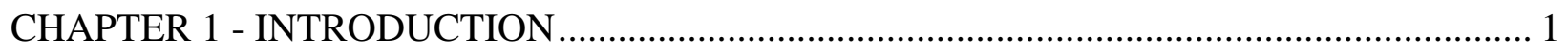

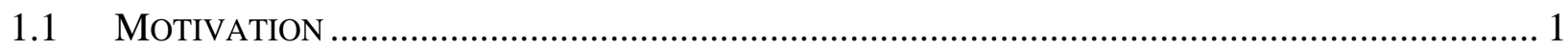

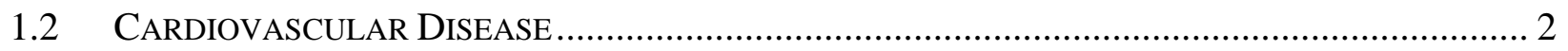

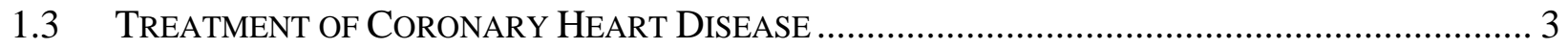

1.3.1 Coronary Artery Bypass Grafting ........................................................................ 3

1.3.2 Angioplasty and Stenting ............................................................................ 7

1.4 Tissue EngineERED VASCULAR GRAFTS (TEVGS)....................................................... 10

1.4.1 History of Tissue Engineering ………………………….................................... 11

1.4.2 Mimicking the Human Blood Vessel.................................................................. 12

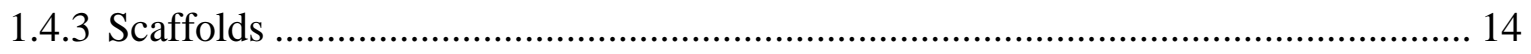

1.4.4 Progress in TEVG Development …………………………............................. 16

1.4.5 Blood Vessel Mimic as an In Vitro Vessel Model................................................. 17

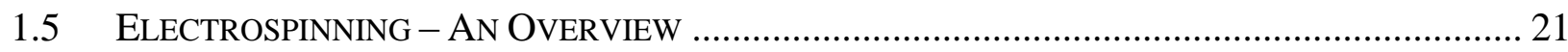

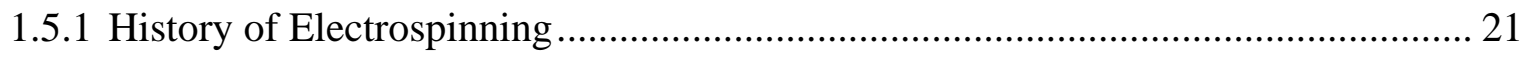

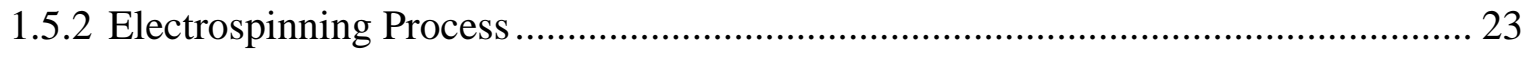

1.5.3 Optimization of Processing Parameters ……………………................................. 25

1.5.3.1 Applied Voltage ……………………………................................. 25

1.5.3.2 Solution Viscosity …………………………..................................... 26

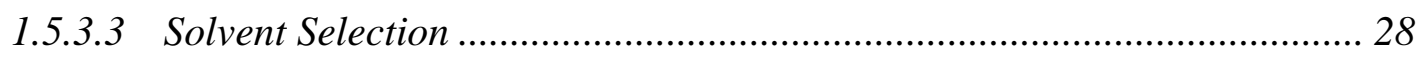

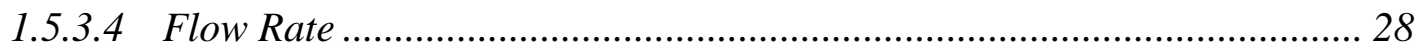




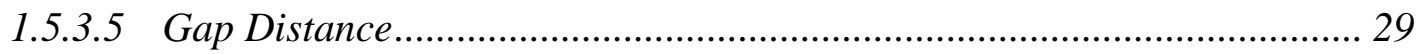

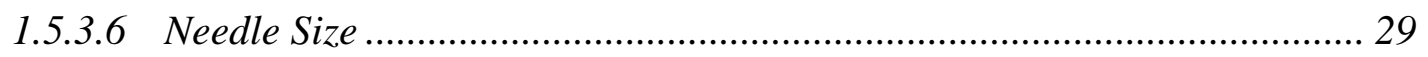

1.5.3.7 Fiber Diameters and Porosity ............................................................... 31

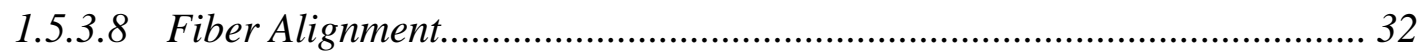

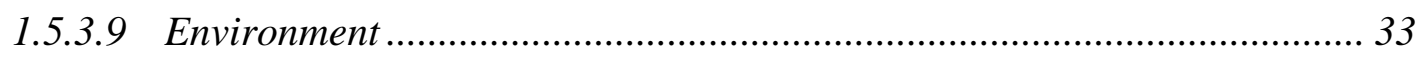

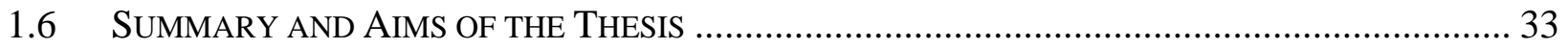

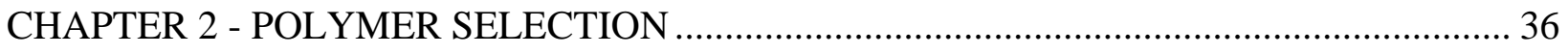

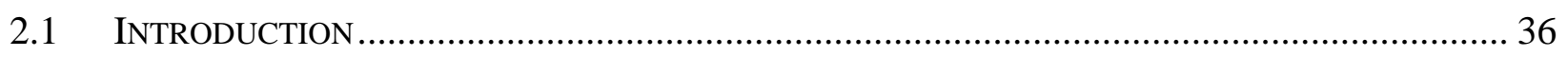

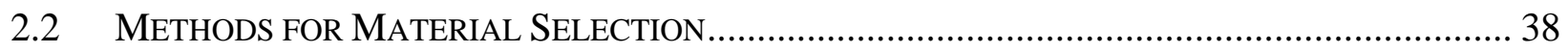

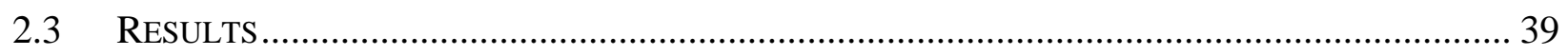

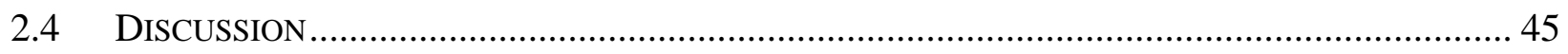

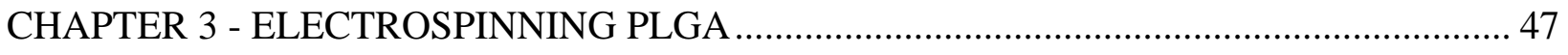

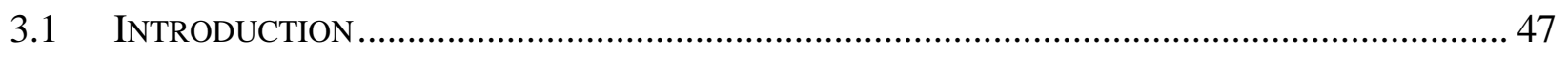

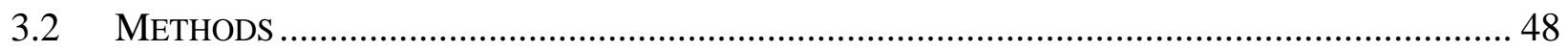

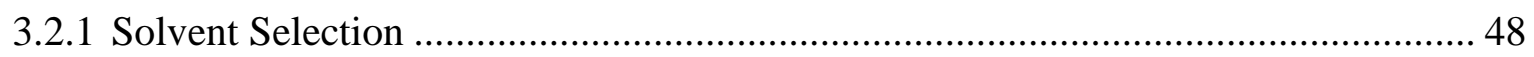

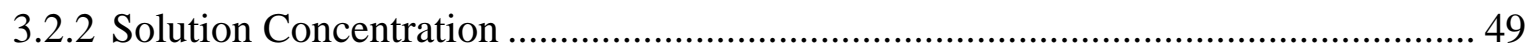

3.2.2.1 Morphological Analysis ................................................................... 50

3.2.3 Processing Parameter Optimization ........................................................................ 51

3.2.3.1 Fiber Diameter Measurements............................................................. 52

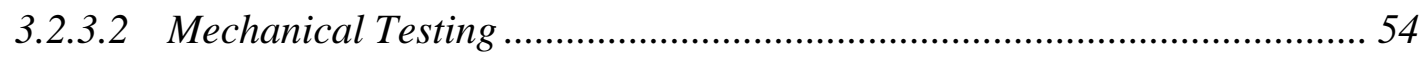

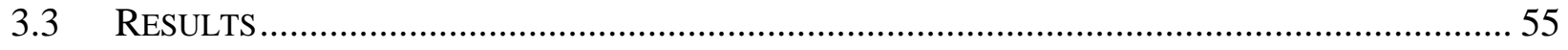

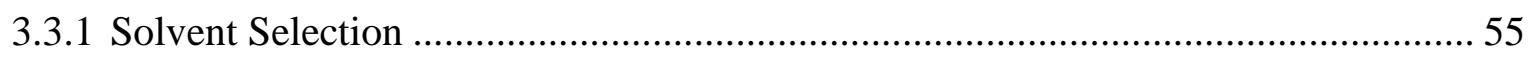

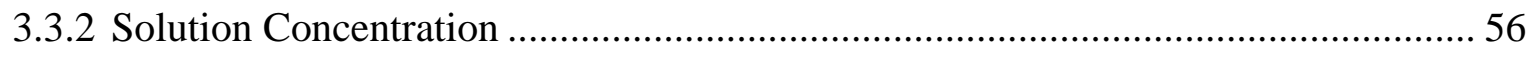


3.3.2.1 Morphological Analysis .................................................................... 58

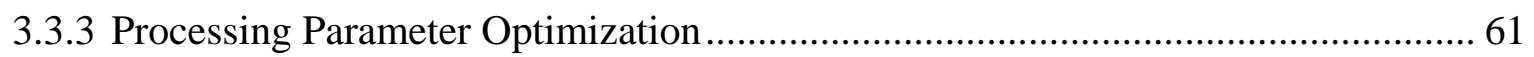

3.3.3.1 Fiber Diameter Measurements............................................................ 62

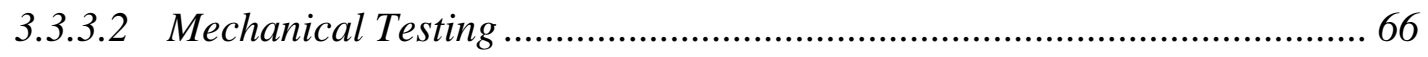

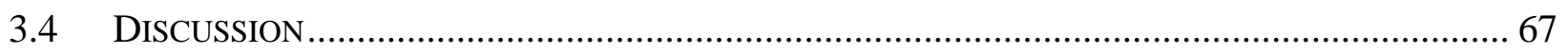

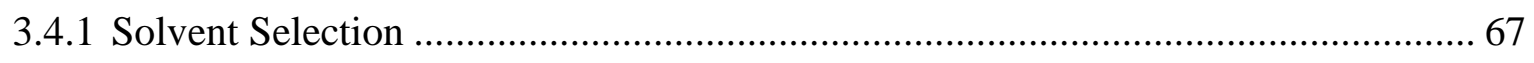

3.4.2 Solution Concentration ……………................................................................. 68

CHAPTER 4 - EVALUATION OF ELECTROSPUN PLGA FOR THE BVM.......................... 73

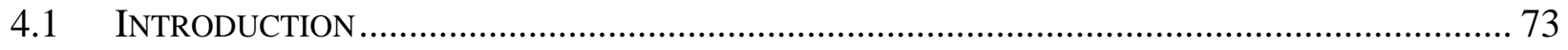

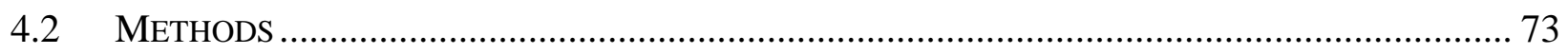

4.2.1 PLGA Response to Media.................................................................................. 73

4.2.2 Morphological Analysis of Samples from Flow Experiments................................. 75

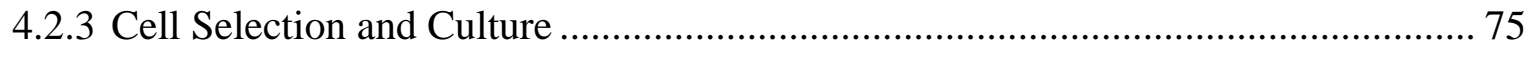

4.2.4 Preparation of Bioreactors and PLGA Scaffolds ..................................................... 76

4.2.5 BVM Bioreactor Set-up with HUVECs.................................................................. 77

4.2.6 Harvesting of PLGA Scaffolds with HUVECs........................................................ 79

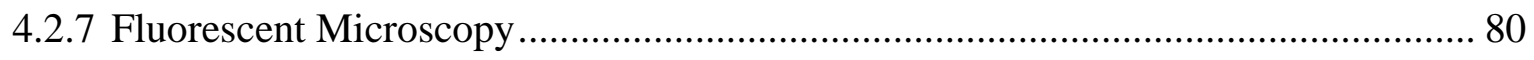

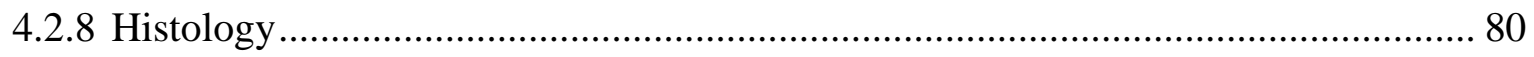

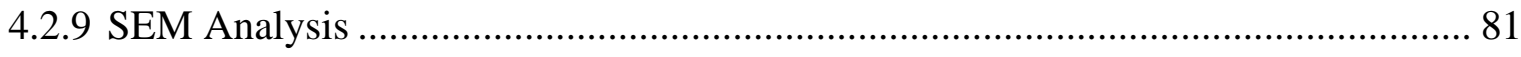

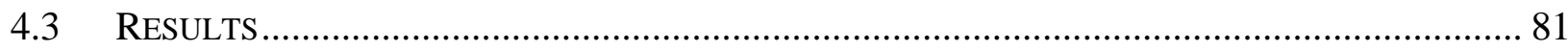

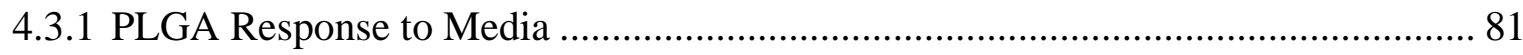

4.3.2 Morphological SEM Analysis of PLGA under Flow …………............................... 82

4.3.3 Preparation of Bioreactors and PLGA Scaffolds .................................................... 83 


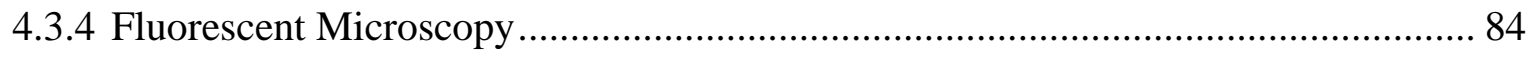

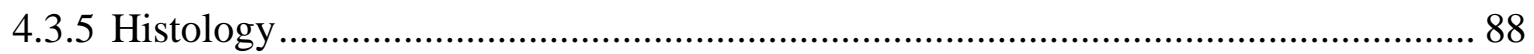

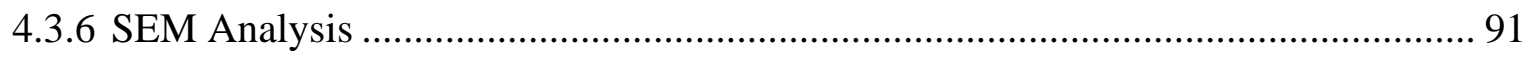

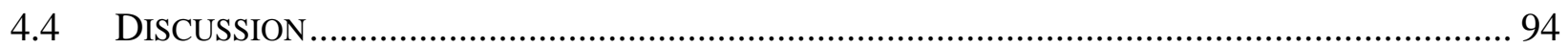

4.4.1 PLGA Response to Media ................................................................................. 94

4.4.2 Results of Electrospun PLGA Cultured with HUVECs .......................................... 96

CHAPTER 5 - DISCUSSION AND CONCLUSIONS ............................................................ 101

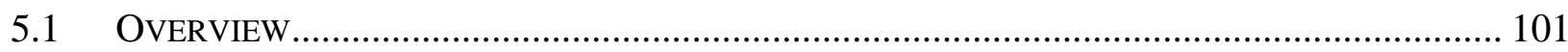

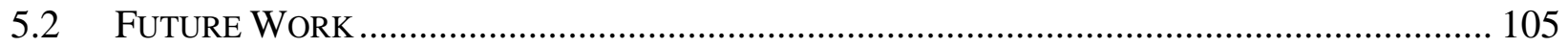

5.2.1 Refinement of Electrospinning PLGA Protocol ……………………………….... 105

5.2.2 Improving the Electrospinning Apparatus ............................................................ 106

5.2.3 Summary of Continuing Investigations .............................................................. 107

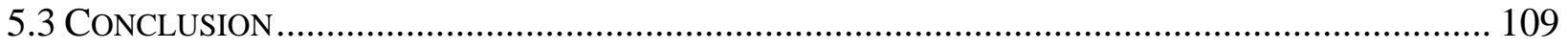

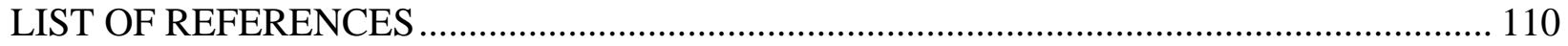

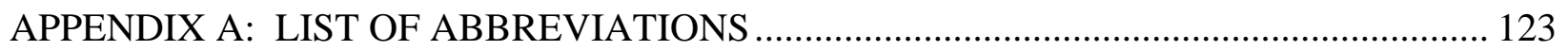

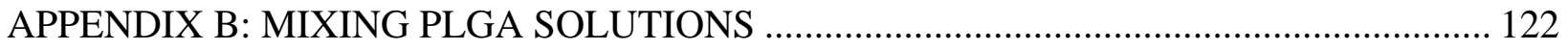

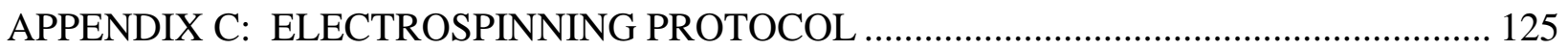

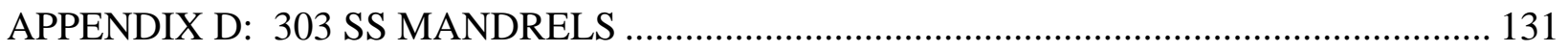

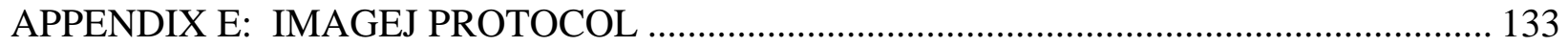

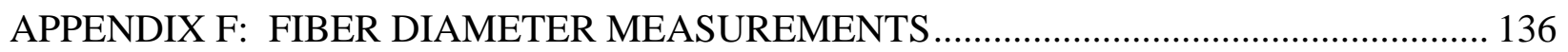

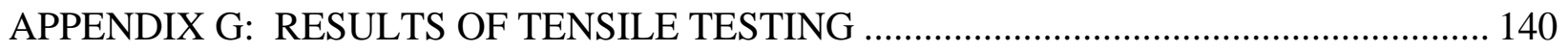




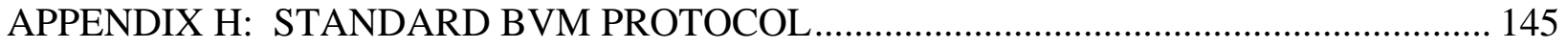

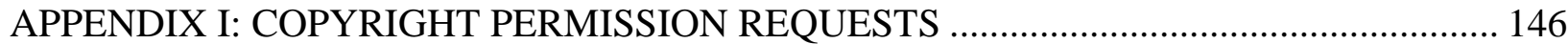




\section{List of Tables}

Table I - Summary Table of Literature Review on Electrospinning Polymer Constructs ........... 41

Table II -Summary Table of Literature Review on Electrospinning Polymer Constructs.......... 43

Table III - Solution and Electrospinning Parameters for PLGA in Chloroform ........................ 50

Table IV - Electrospinning Process Parameters for 15 wt\% PLGA in $\mathrm{CHCl}_{3} \ldots \ldots \ldots \ldots \ldots \ldots \ldots . . . . . . . . . . .22$

Table V - Price Comparisons for Different Solvents...................................................... 55

Table VI - Fiber Diameter Results for 15 wt\% PLGA Electrospun Scaffolds .......................... 63

Table VII - Mechanical Properties of 15 wt\% PLGA......................................................... 66

Table VIII - Summary Table of HUVEC Sodded PLGA Scaffolds ...................................... 85 


\section{List of Figures}

Figure 1 - Atherosclerosis is the buildup of plaque along the inner arterial wall....................... 3

Figure 2 - The above illustration shows a bypass graft rerouting blood flow around ................... 4

Figure 3 - The figure shows a cross-section of the coronary artery with plaque buildup............. 8

Figure 4 - The figure shows a cross-section of the coronary artery with plaque buildupstent is

mounted onto a balloon and inserted into the diseased region of the artery .............................. 9

Figure 5 - The Blood vessel mimic (BVM) system above is made of a polymer...................... 18

Figure 6 - The leak proof polypropylene container allows more room for scaffold insertion...... 20

Figure 7 - A schematic representation of a typical electrospinning setup.............................. 23

Figure 8 - The scanning electron microscope image of ePTFE. .............................................. 37

Figure 9 - The above images are example images and do not display SPIN $1-5 \ldots \ldots \ldots \ldots \ldots \ldots . . . .51$

Figure 10 - ImageJ can be used to convert pixels into a length scale..................................... 53

Figure 11 - Illustration of section A-B from each SPIN prepared for tensile testing .................. 54

Figure 12 - SEM image of SPIN 1, $10 \mathrm{wt} \%$ PLGA. ................................................................. 59

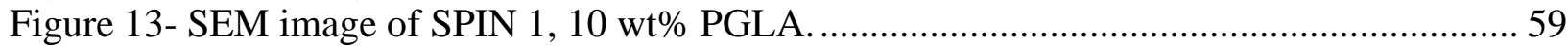

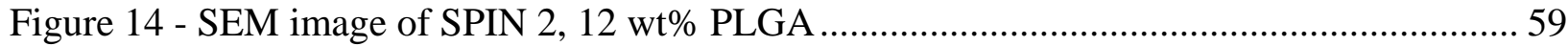

Figure 15 -SEM image of SPIN 3, 13 wt\% PLGA ............................................................. 59

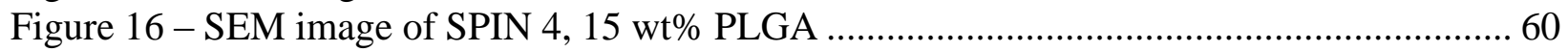

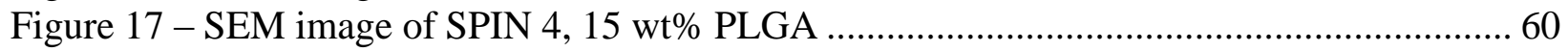

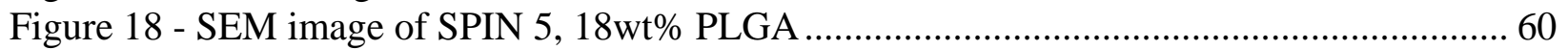

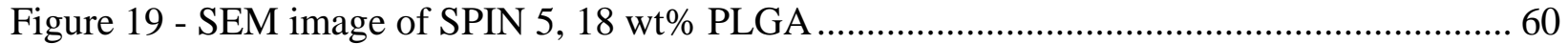

Figure 20 - SEM image of SPIN 6, lumen of PLGA electrospun construct (2000X)................ 64

Figure 21 - SEM image of SPIN 6, albumen of PLGA electrospun construct (610X)............... 64

Figure 22 - SEM image of SPIN 7, lumen of PLGA electrospun construct (2000X)............... 64

Figure 23 - SEM image of SPIN 7, ablumen of PLGA electrospun construct (800X)............... 64

Figure 24 - SEM image of SPIN 8, lumen of PLGA electrospun construct .............................. 65

Figure 25 - SEM image of SPIN 8, ablumen of PLGA electrospun construct (800X)............... 65

Figure 26 - SEM image of SPIN 9, lumen of PLGA electrospun construct .............................. 65

Figure 27 - SEM image of SPIN 9, ablumen of PLGA electrospun construct (800X)............... 65

Figure 28 - The above picture shows both BVM chambers containing PLGA ....................... 75

Figure 29 - Preparation of BVM chambers for cell sodding in a sterile environment................. 77

Figure 30 - Final setup of BVMs in incubator and hooked up to peristaltic pump. ................... 78

Figure 31 - Image of media color changes due to acidic by-products of PLGA in static media. . 82

Figure 32 - The above SEM image was taken of the lumen of a PLGA …............................ 83

Figure 33 - Original electrospun scaffold is shown on the left............................................ 84

Figure 34 - Images of BVM01 cultured with HUVECS for 24 hours under flow..................... 85

Figure 35 - Images from BVM02 cultured with HUVECs for 3 days ...................................... 86

Figure 36 - Images of BVM03 show cells present for both distal (left) ................................ 87

Figure 37 - Images of BVM04, both proximal (left) and distal (right)................................. 87

Figure 38 - The above image from BVM03 shows the fibrous nature of the PLGA ................ 88

Figure 39 - Histology images of BVM03 shows cluster of cells and a damaged sample............ 89

Figure 40 - Histology shows the lumen of BVM04 was closed during processing..................... 90

Figure 41 - Histology images of BVM01 show a cellular lining on the lumen of the vessel..... 91

Figure 42 - HUVEC sodded PLGA scaffold after 24 hrs continuous flow culture at 15 RPM... 92

Figure 43 - HUVEC spreading shown on the lumen of the electrospun PLGA ....................... 93 
Figure 44 - Cross sectional image of BVM02 showing the fibrous nature .............................. 94

Figure 45 - A 5g bottle of PLGA from Sigma................................................................ 123

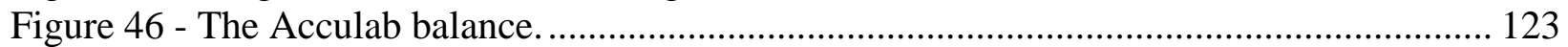

Figure 47 - A 1 liter bottle of chloroform...................................................................... 123

Figure 48 - Pipette aid for obtaining solvent. .............................................................. 123

Figure $49-20 \mathrm{ml}$ vial with solution and label. ........................................................... 123

Figure 50 - Orbital shake table for mixing solutions. ..................................................... 124

Figure 51 - Green ground wire on back of collector......................................................... 125

Figure 52 - Yellow power wire connects DC control box.................................................. 125

Figure 53 - Entire collector...................................................................................... 125

Figure 54 - Polymer residue on wires...................................................................... 126

Figure 55 - Checking resistance between mandrel and ground wire.................................. 127

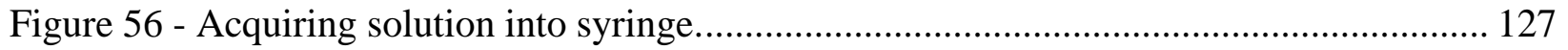

Figure 57 -Positioned syringe in the pump. ................................................................. 127

Figure 58 - Proper positioning of the collector............................................................. 128

Figure 59 - Red wire hung on needle....................................................................... 128

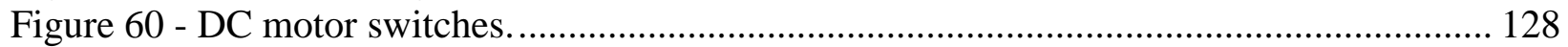

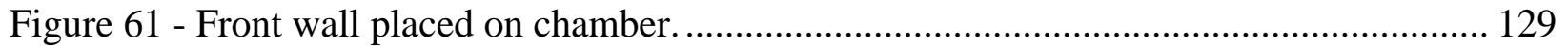

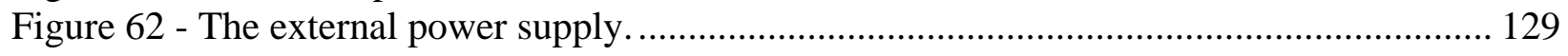

Figure 63 - The Main Power switch. ........................................................................... 129

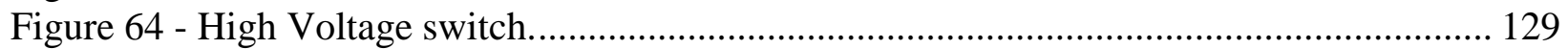

Figure 65 - Tensile strength for SPIN 4, 15 wt\% PLGA in $\mathrm{CHCl}_{3}(5.5 \mathrm{ml} / \mathrm{hr}$; unknown)........ 140

Figure 66 - Longitudinal Young's modulus for SPIN 4, 15 wt\% PLGA in $\mathrm{CHCl}_{3} \ldots \ldots \ldots \ldots \ldots . . . . . .140$

Figure 67 - Circumferential Young's modulus for SPIN 4, 15 wt\% PLGA in $\mathrm{CHCl}_{3} \ldots \ldots \ldots \ldots . . . . .140$

Figure 68 - Tensile strength for SPIN 6, 15 wt\% PLGA in $\mathrm{CHCl}_{3}(5.5 \mathrm{ml} / \mathrm{hr} ; 12,000 \mathrm{~V}) \ldots \ldots \ldots 141$

Figure 69 - Longitudinal Young's modulus, SPIN 6, 15 wt\% PLGA in $\mathrm{CHCl}_{3} \ldots \ldots \ldots \ldots \ldots \ldots . . . . . . . .141$

Figure 70 - Circumferential Young's modulus, SPIN 6, 15 wt\% PLGA in $\mathrm{CHCl}_{3} \ldots \ldots \ldots \ldots \ldots . . . . .141$

Figure 71 - Tensile strength for SPIN 7, 15 wt\% PLGA in $\mathrm{CHCl}_{3}(3.0 \mathrm{ml} / \mathrm{hr} ; 12,000 \mathrm{~V}) \ldots \ldots . .142$

Figure 72 - Circumferential Young's modulus, SPIN 7, 15 wt\% PLGA in $\mathrm{CHCl}_{3} . \ldots \ldots \ldots \ldots \ldots . . . . . .142$

Figure 73 - Tensile strength for SPIN 8, 15 wt\% PLGA in $\mathrm{CHCl}_{3}(3.0 \mathrm{ml} / \mathrm{hr} ; 15,600 \mathrm{~V}) \ldots \ldots . .143$

Figure 74 - Longitudinal Young's modulus, SPIN 8, 15 wt\% PLGA in $\mathrm{CHCl}_{3} \ldots \ldots \ldots \ldots \ldots \ldots . . . . . . .143$

Figure 75 - Circumferential Young's modulus, SPIN 8, 15 wt\% PLGA in $\mathrm{CHCl}_{3} \ldots \ldots \ldots \ldots \ldots . . . . .143$

Figure 76 - Tensile strength for SPIN 9, 15 wt\% PLGA in $\mathrm{CHCl}_{3}(5.5 \mathrm{ml} / \mathrm{hr} ; 15,600 \mathrm{~V}) \ldots \ldots . .144$

Figure 77 - Longitudinal Young's modulus for SPIN 9, 15 wt\% PLGA in $\mathrm{CHCl}_{3} \ldots \ldots \ldots \ldots \ldots . . . . . .144$

Figure 78 - Circumferential Young's modulus for SPIN 9, 15 wt\% PLGA in $\mathrm{CHCl}_{3} \ldots \ldots \ldots \ldots . . . . .144$ 


\section{Chapter 1 - Introduction}

\subsection{Motivation}

The introduction of stents in 1987 for the treatment of atherosclerosis has drastically increased the effectiveness of traditional balloon angioplasty [1]. Since that time, stents have and are continually being developed and improved with advancements in areas of architecture, drug-eluting designs, deployment methods, and adjuvant drug therapies [2]. With each new design, the need for fast, reliable data is imperative to move these devices to the market. Although in vivo animal models for stenting and angioplasty have been developed and extensively utilized, time, money and animal numbers could be reduced by implementing a test model that simulates the human physiological condition in vitro.

An in vitro "blood vessel mimic" (BVM) that utilizes tissue engineering techniques has been demonstrated as a method for evaluating endothelialization of bare metal stents [3]. Although the primary focus of tissue engineered vascular grafts (TEVGs) has been replacement and bypass therapy for damaged or blocked blood vessels, tissue engineering blood vessels for the BVM is a promising alternative application that could have substantial impact on early intravascular device evaluation and development prior to animal and clinical trials.

The current BVM model has several physiological and mechanical limitations as well as scale up limitations. Presently, the BVM is made of a biocompatible, non-biodegradable expanded poly(tetrafluoroethylene) [ePTFE] scaffold. The scaffold is critical to proper tissue development and simulation of the native vessel, but there are several disadvantages associated with ePTFE. Disadvantages include high cost, poor mechanical compliance and inability to tailor morphological and mechanical properties specific for the BVM application. Consequently, 
the aim of this thesis is to determine an alternative scaffolding material that could improve the BVM model and eliminate some of the current scaffolding limitations.

The following sections of this Introduction will provide the background and foundation on which this thesis is based. A review of coronary artery disease and treatments, including bypass grafting, angioplasty, and stenting will be presented. A summary of TEVGs and the importance of scaffolding for successful construct development, as well as the application of tissue engineered blood vessels for in vitro device evaluation, will be provided. The Introduction will include a review of electrospinning, the polymer scaffold fabrication technique utilized in this thesis, including history, theories, and process parameter optimization specifically for TEVG development. The Introduction will conclude with a summary of the overall goals and objectives for this thesis.

\subsection{Cardiovascular Disease}

According to the 2009 American Heart Association's (AHA's) update on Heart Disease and Stroke Statistics, 1 in 3 adult Americans (approximately 80 million people) are currently affected by 1 or more types of cardiovascular diseases (CVDs). Leading as the number one cause of death in America, CVD is responsible for 2,400 fatalities per day which corresponds to 1 death every 37 seconds in the United States [4].

Coronary artery disease (CAD), also known as coronary heart disease (CHD), caused 1 in 5 deaths in the United States in 2005 and is the leading CVD killer for all American men and women. In 2008 the cost for treating CHD was estimated at $\$ 156.4$ billion [4]. CHD is strongly equated with atherosclerosis (Figure 1), which occurs when coronary arteries are blocked by plaque, thus restricting necessary blood flow and leading to angina, shortness of breath, heart 
attack, stroke and often death [5]. Device based treatments for CHD include bypass grafting, angioplasty and stenting.

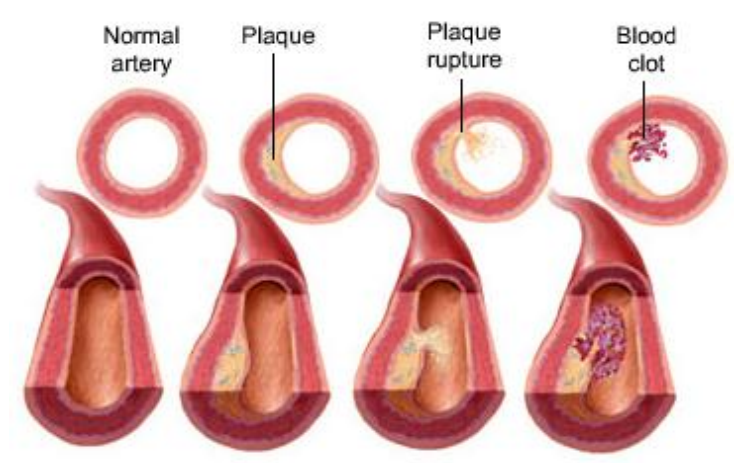

Figure 1 - Atherosclerosis is the buildup of plaque along the inner arterial wall. Unstable plaque can rupture causing blood clots leading to heart attack or death.

(Permission granted to reprint image from medmovie.com a division of Gersony Medical, Inc; See Appendix I)

\subsection{Treatment of Coronary Heart Disease}

Coronary heart disease can be treated by lifestyle modifications, medications and medical procedures. The goal of each aforementioned prescribed treatment is to alleviate symptoms, slow the progression of disease, and repair or replace diseased tissue, respectively. A brief introduction to coronary artery bypass procedures, angioplasty, and stenting will be provided within this section. The purpose of this section is to introduce the reader to CHD treatments as a necessary foundation for intravascular device testing and tissue engineering.

\subsubsection{Coronary Artery Bypass Grafting}

Nearly 448,000 coronary artery bypass grafts (CABG) surgeries are conducted each year [4]. Coronary bypass surgery treats atherosclerosis by bypassing the diseased area using a natural or synthetic graft to reroute blood flow (Figure 2). The procedure is highly invasive, 
expensive and is associated with a long recovery time; however, CABG is highly successful long term, with lower mortality rates, less angina, and fewer revascularization procedures compared to alternative methods [6].

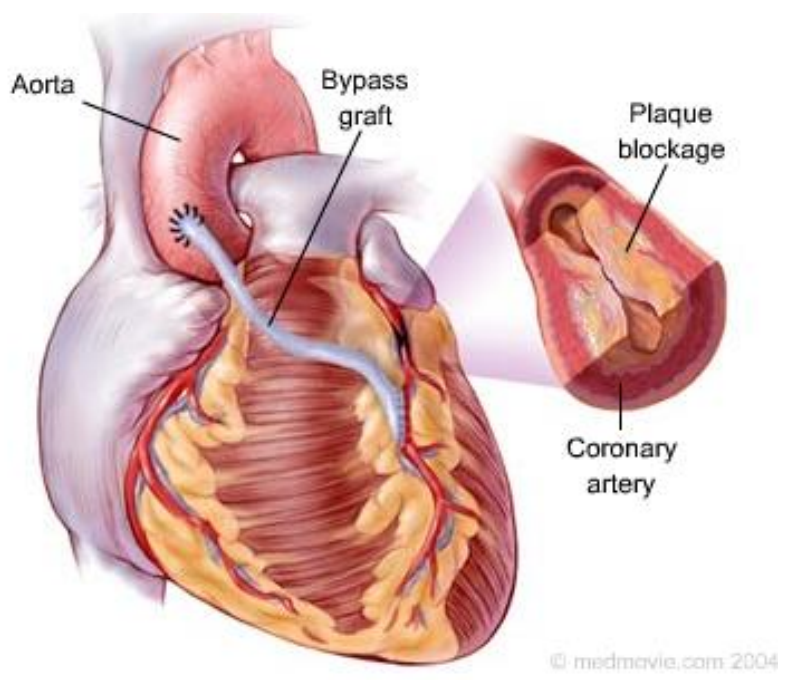

Figure 2 - The above illustration shows a bypass graft rerouting blood flow around the diseased region of the coronary artery. The graft is connected from the aorta to the distal part of the epicardial coronary arteries.

\section{(Permission granted to re-print image from medmovie.com a division of Gersony Medical Media, Inc.; See Appendix I)}

Biological vascular grafts can be classified as autografts, allografts or xenografts. Autografts are transplanted grafts from one part of the body to another within the same individual. The standard choice of autologous grafts for CABG surgery is either the internal mammary artery (IMA) or the greater saphenous vein [7], however the radial artery and lesser saphenous vein and others have been used. IMA grafts are superior to saphenous vein grafts (SVG) with twice the patency for a 10 year period $[7,8]$. During coronary bypass surgery, the replacement vessel is cut out of the chest, arm, or leg and is connected from the side of the coronary artery to the aorta, as shown above in Figure 2. Autografts will not likely elicit an 
immune response and therefore, are the optimum choice for bypass surgery. However, patients needing replacement therapy often lack the necessary healthy autologous vessel. An estimated $60 \%$ of all patients in need of bypass surgery do not have a vessel suitable for grafting, mainly due to pre-existing diseases and conditions, such as, intrinsic venous disease, varicose vein ligation, prior myocardial revascularization and previous peripheral arterial reconstruction [9]. Consequently, if no suitable autologous conduit exists, alternative non-autologous conduits can be used.

Allografts are donated vessels from one individual to another of the same species. Allografts present high risks associated with immune rejection, but a close genetic match can increase patency. Patients who receive donor constructs will remain on immunosuppressive drugs to prevent the chance of transplant rejection. Unfortunately, the number of graft donors does not meet the high demand for replacement conduits, and often those on the wait list die before an acceptable donor has been matched [10]. This severe shortage in donor tissue has lead to the exploration of xenografts.

Xenografts are donated vessels from a different species. The genetic disparity between human host and xenograft is maximized and such grafts will elicit rejection immediately. Small progress has been made with porcine vessels, which are most similar to the human vessel in physiology and size [11]. Still, with the implementation of xenografts the need for immunosuppressive drugs, in order to stop immediate rejection by the host, is a costly and crucial necessity. With the many limitations associated with biological grafts for coronary artery bypass surgery, synthetic grafts have been developed as a possible alternative to alleviate symptoms and to treat CAD. 
Advantages of synthetic grafts over biologic grafts as replacement conduits include: cost, availability, and the potential to tailor physical properties to match patient conditions (e.g. size and shape). The most widely used synthetic biomaterials for peripheral bypass surgery are poly(ethylene terephthalate) [PET or Dacron] and expanded poly(tetrafluoroethylene) [ePTFE or GORE-TEX®], both of which have been clinically tested with successful outcomes for large diameter (> $6 \mathrm{~mm})$ vascular grafts. However, these synthetic replacements in small diameter sites, with lower blood flow velocities, have resulted in thrombosis rates of nearly $40 \%$ in just a few months [10-13]. The primary disadvantages of synthetic grafts leading to graft failure are the lack of an appropriate endothelium on the lumen of the graft, and compliance mismatch [12]. Often, compliance mismatch in synthetic vascular grafts is associated with the onset of intimal hyperplasia and aneurysm development, resulting in graft failure [13-16]. Consequently, in vitro surface endothelialization of Dacron and ePTFE has been shown to significantly increase the anti-thrombogenicity of grafts in vivo $[17,18]$. Other experimental methods of increasing synthetic vascular graft patency include heparinization, chemical modifications, and various protein coatings [19-21]. Although there have been significant improvements in these grafts, surface modifications in each of the aforementioned studies typically lead to clotting, intimal hyperplasia, and ultimately, bypass graft failure $[17,19,20]$. Still, compliance mismatch with ePTFE and PET has not been resolved. Other major complications sometimes associated with synthetic grafts include infection, periprosthetic fluid collection, and structural degeneration [22]. Finally, both natural and synthetic grafts often require revision surgery [23]. Limitations in natural and synthetic grafts and the invasiveness of CABG surgery make angioplasty a highly desirable alternative treatment to CHD. 


\subsubsection{Angioplasty and Stenting}

Percutaneous coronary intervention (PCI), or angioplasty, treats atherosclerosis by inflating a balloon inside the blocked region of the artery to push or break the plaque against the arterial wall, reopening the lumen for blood to flow (Figure 3). The balloon is inserted through the use of a catheter usually by way of the femoral artery at the groin. Balloon angioplasty is a minimally invasive procedure compared to bypass surgery; however, angioplasty is highly subject to re-narrowing of the arterial wall, most commonly referred to as restenosis. According to the National Institute of Health, 4 out of 10 patients develop restenosis after angioplasty [24]. Restenosis is believed to be the result of elastic recoil of the arterial wall and the formation of scar tissue due to vessel injury from balloon inflation [25]. 


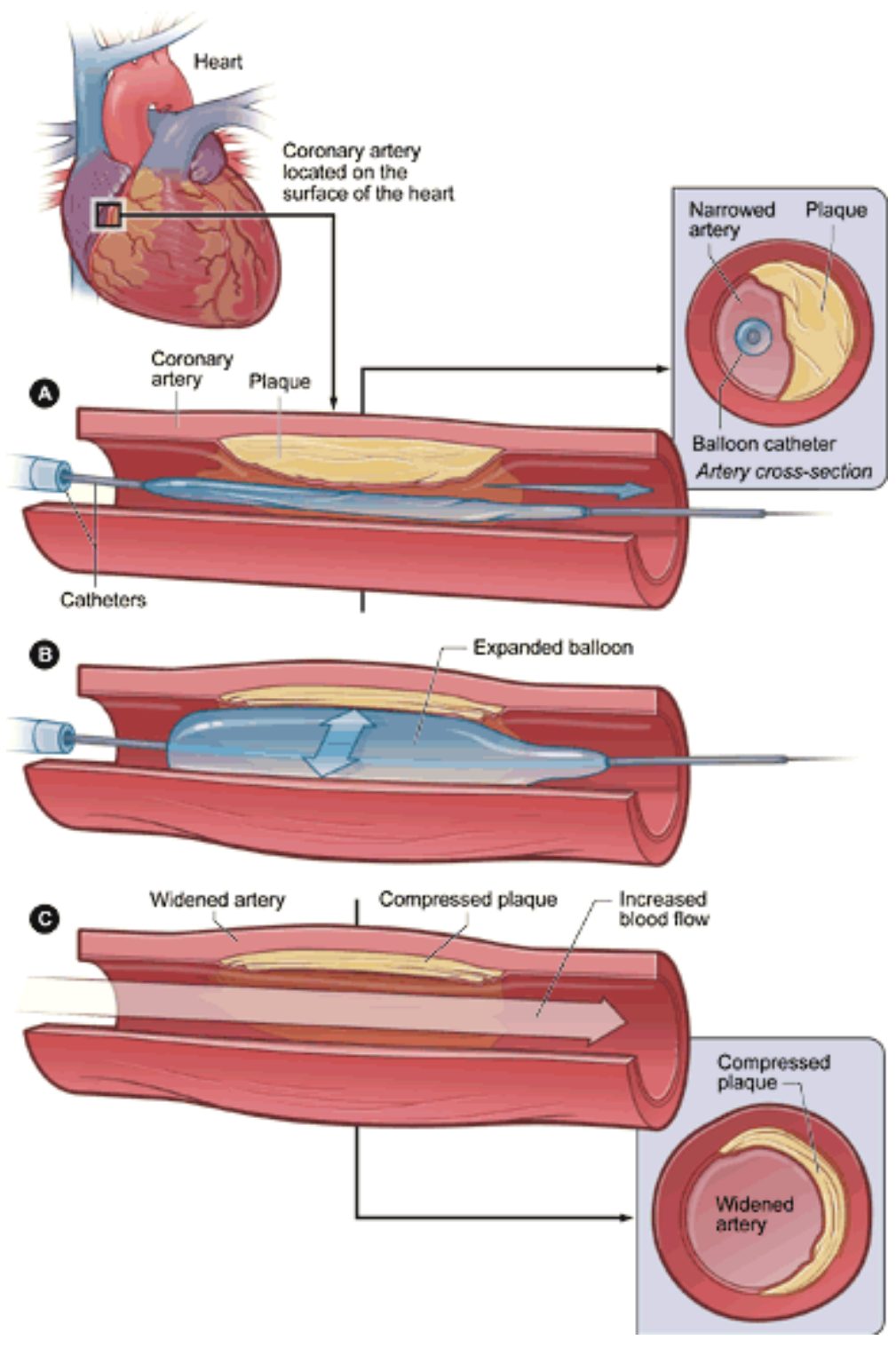

Figure 3 - The figure shows a cross-section of the coronary artery with plaque buildup. A deflated balloon is first inserted by way of a catheter into the diseased region (A). The balloon is then inflated, pressing plaque against the arterial wall $(\mathrm{B})$. The balloon is removed and the widened artery restores blood flow $(\mathrm{C})$.

(Permission granted to re-print image from the National Heart, Lung, and Blood Institute as a part of the National Institute of Health and the U.S. Department of Health and Human Services; See Appendix I)

Stenting is the process of deploying a wire mesh tube, or stent, to open an artery during balloon angioplasty (Figure 4). The stent typically remains in the artery permanently to support blood flow and acts as a scaffold for endothelialization. Stenting was first incorporated into PCI 
procedures in 1987 and since then approximately $80 \%$ of all angioplasties are combined with stenting due to an outstanding reduction in restenosis rates from $30-40 \%$ to $15-20 \%$ [1].

Specifically, stenting reduces restenosis by minimizing elastic recoil of the vessel, in addition to providing an anti-thrombogenic surface for blood to flow $[1,26]$

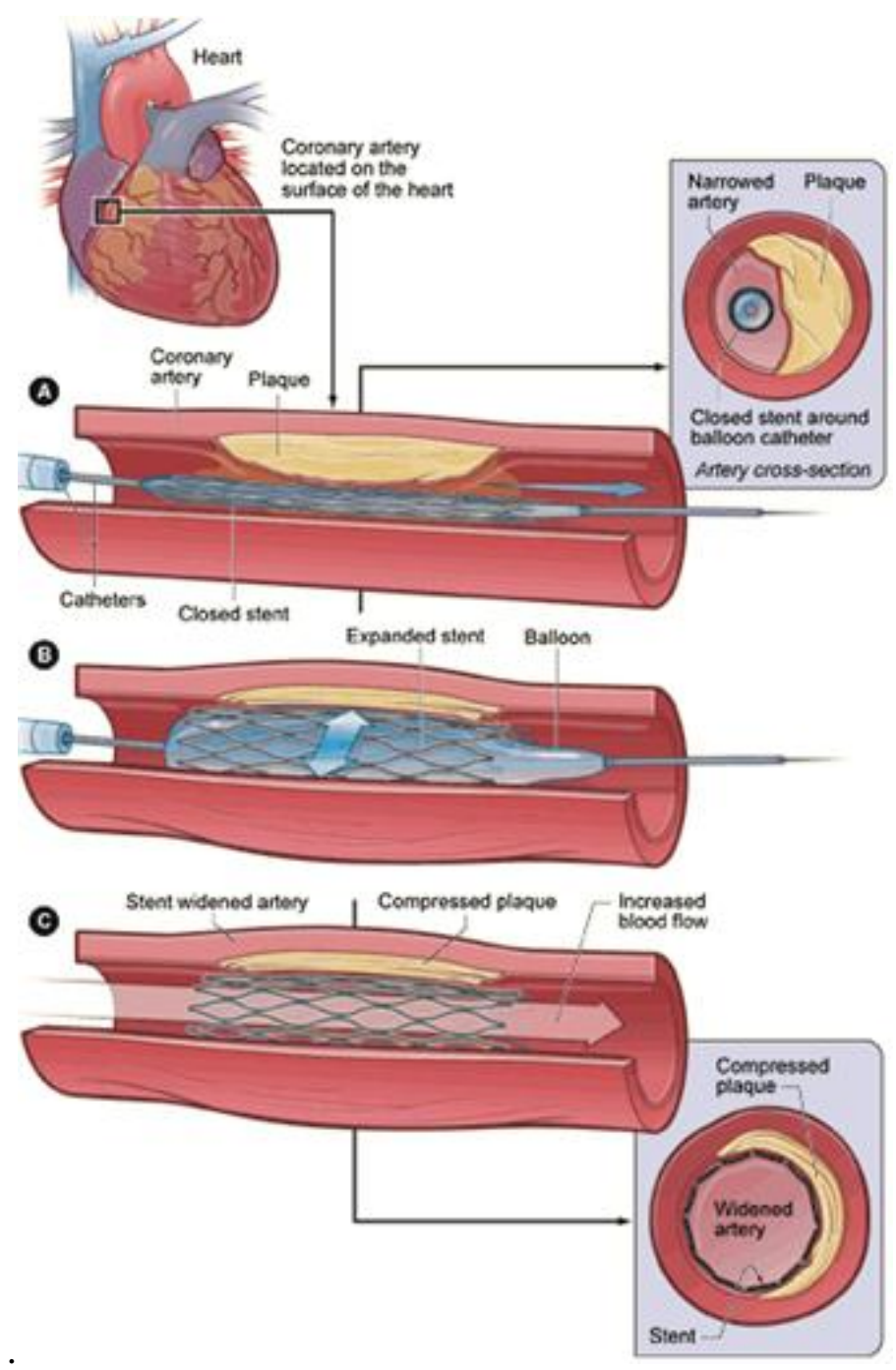

Figure 4 - The figure shows a cross-section of the coronary artery with plaque buildup. The stent is mounted onto a balloon and inserted into the diseased region of the artery by way of a catheter (A). The balloon and stent together are expanded, pushing the plaque against the arterial wall $(B)$. The stent remains as a permanent support in the vessel and the balloon is removed. The widened artery restores blood flow (C).

(Permission granted to re-print image from the National Heart, Lung, and Blood Institute as a part of the National Institute of Health and the U.S. Department of Health and Human Services; See Appendix I) 
Serious complications remain associated with stenting, such as increased thrombosis and in-stent restenosis [25]. Increased thrombosis has been largely controlled through the use of anti-platelet therapy regimes with such drugs as aspirin, warafin, and heparin [27]. In-stent restenosis is explained as the arterial healing response due to balloon and stent expansion during angioplasty with a stent, in which case the blood vessel is damaged and consequently initiates cellular proliferation and intimal hyperplasia [1]. Given that the stent is typically permanent, introduction of the stent into the body leads to localized inflammatory responses and foreign body reactions that contribute to restenosis as well [28]. Many efforts have been made to reduce in-stent restenosis, with most recent efforts focusing upon drug-eluting and bioabsorbable stents.

The goal of drug-eluting stents is to prevent narrowing of the arterial wall through the continuous release of medications or bioactive agents bound in the stent coating or matrix after implantation [29]. Bioactive agents are released into the blood stream and locally in tissue surrounding the stent [29]. Several advancements have been made and are continually being pursued and studied, including stent design, biodegradable polymers, coatings, medications, drug release rates and homogeneity, and dosages $[1,30]$. With each new development, the need for intravascular device testing increases. Given the high costs and controversy over validity of preclinical animal testing, a more physiological in vitro testing system could provide faster and more reliable results prior to in vivo analysis. Such a system will be described later in the Introduction of this thesis.

\subsection{Tissue Engineered Vascular Grafts (TEVGs)}

With the optimization of small diameter synthetic grafts far from ideal and the limited availability of autologous and allogenic vascular grafts, tissue engineering has emerged as a 
means of potentially creating completely autologous tissue engineered blood vessels for small caliber vascular grafts. This section of the Introduction will provide a basic overview of tissue engineering, specifically TEVGs as an alternative to standard CABGs. A more detailed review of the importance of scaffolding during the development of TEVGs will be provided. The end of this section will introduce the reader to previous work on a TEVG-based in vitro testing system for intravascular devices. The system was developed through the concept of tissue engineering blood vessels and is the primary application for the work of this thesis.

\subsubsection{History of Tissue Engineering}

The concept of tissue engineering was first applied in the 1980's and since then the number of tissue engineered applications and investigations has increased with enthusiasm among both engineers and scientists. Many tissue engineered constructs have undergone clinical trials and some are already on the market. Areas of tissue engineering exploration involve fabricated skin [31], liver [32], pancreas [33], intestines [34], esophagus [35, 36], nerves [37], cartilage [38-40], bones [40, 41], ligaments [42, 43] and tendons [43, 44]. Significant contributions have also been made in the cardiovascular area, specifically valve leaflets [45, 46] and blood vessels [47-50].

Tissue engineering can be defined as the combination of engineering and biologic principles to generate or repair tissue or organs through the cultivation of cells on a scaffold in vitro, for transplantation into the appropriate anatomical region of a diseased patient [22]. The goal of tissue engineering is to recreate or mimic human tissue, either in whole or in part, in vitro, using autologous cells. Ideally, using the patient's own cells will allow the construct to function appropriately without rejection. In theory, cells are isolated from the patient and 
expanded in cell culture to be seeded onto a scaffold or artificial extracellular matrix (ECM). Scaffold and cells together are then typically matured in a bioreactor, which is a device used to transport nutrients and oxygen, maintain concentration levels, impart mechanical and electrical stimulus to imitate the body, control environmental conditions (e.g. $\mathrm{pH}$, temperature, $\mathrm{CO}_{2}$ levels, growth factors, gradients etc.) and induce homogenous cell distribution. Barron et al.

generalized the bioreactor as a simulated biological environment where "in vivo biomechanical and biochemical conditions are created in vitro for functional tissue development" [23]. Once the tissue engineered construct has been developed the construct is implanted into the patient where remodeling results in the construct becoming fully functional within the body and surrounding tissues [22].

\subsubsection{Mimicking the Human Blood Vessel}

The need for small-diameter vascular bypass conduits has driven the development of tissue engineered vascular grafts (TEVGs). In order to produce a TEVG, the anatomy, function and properties of the human vessel must be considered. This section will outline the basic anatomy and function of the blood vessel and how each component is simulated or created by general tissue engineering techniques.

There are three distinct layers of arteries; the tunica intima, tunica media, and tunica adventitia, which together surround the vessel lumen, or the space containing blood. The intima, closest to the lumen, is made of a continuous layer of endothelial cells (ECs) responsible for creating a frictionless surface for unhindered blood flow and prohibiting platelet activation. The internal elastic lamina separates the intima from the media. In TEVGs, ECs are harvested from 
vessels or obtained as derivatives of endothelial progenitor cell lines [51] and are used to engineer the luminal layer of cells on a scaffold in vitro.

The media, middle layer, is composed of a dense population of smooth muscle cells (SMCs) within a surrounding ECM. The ECM is composed of collagen types I and III and elastin fibers, and is responsible for vasoconstriction or vasodilatation in order to maintain necessary blood pressure and blood flow. The external elastic lamina separates the media from the adventitia. For a TEVG, the properties of the media may be provided by the scaffold material. However, SMCs can be used for developing the media of a TEVG. L'Heureux et al. cultured sheets of vascular SMCs and placed these sheets around a tubular support device. After maturation the support was removed and endothelial cells were seeded on the lumen, thus producing a scaffold-free functional vessel [52].

The adventitia is largely composed of fibroblasts and an ECM of loosely woven collagen and elastin [53]. Collagen, specifically, provides the stiffness and high tensile strength necessary for vessel protection and structural integrity, whereas elastin, in both the media and adventitia, contributes to the compliance of the vessel in response to arterial pulsation. In general, the scaffold used to tissue engineer blood vessels acts as both the media and adventitia. In some instances, sheets of fibroblasts wrapped around the scaffold have been used to simulate the adventitia [54]. However, in most cases, the scaffold is the major structural component that provides the mechanical properties of both the media and adventitia of the native vessel.

Requirements of TEVGs are not limited to simply mimicking the native blood vessel anatomy. In order to truly mimic the native blood vessel, TEVGs must contain a confluent and adherent layer of ECs and must have the mechanical properties necessary to maintain 
compliance under pulsatile flow and structural integrity under increased pressures, properties provided by elastin and collagen in the ECM of the native vessel [55]. Additional requirements of an ideal TEVG include suture retention, kink resistance, manufacturability, cost-effectiveness, reproducibility in a variety of lengths and diameters, easy storage, and the ability to be produced in a reasonable amount of time [56].

\subsubsection{Scaffolds}

The interplay of cells and scaffolds is a critical component of tissue engineering. The cellular component is responsible for the construct performing the same function as that of the tissue being replaced. Cells are encouraged to develop into the proper phenotype and orientation largely by the scaffold they interact with and the environment in which they are maintained. This section is aimed at providing an overview of scaffold design for tissue engineered vascular constructs. Details on scaffold morphology and mechanical behavior and the corresponding effects on cell interactions and final construct endpoints will be provided.

In general, tissue engineering utilizes a synthetic or natural, biodegradable three dimensional (3D) microporous scaffold, which is then cultivated with cells in a bioreactor in until a tissue construct is formed. The most basic function of the scaffold is to provide mechanical stability and the overall shape, size and volume of the tissue construct being developed [57]. Scaffold morphologies are largely responsible for encouraging and promoting cellular adhesion and correct phenotype, thus guiding tissue development into the appropriate construct. Generally, a biodegradable scaffold is desired such that all implanted materials will eventually be fully incorporated into the body and no foreign material will remain. In such cases, resorption and degradation kinetics must be in balance with development and remodeling 
capabilities of the cells in vivo or in vitro. However, non biodegradable scaffolds are also desirable in cases where structural integrity must be maintained or when the host's body is unable to form the necessary replacement tissue. Degradation bi-products also play a role in the success and biocompatibility of a scaffold. For a scaffold to be biocompatible, it must not elicit or encourage an undesirable tissue response or interrupt any physiological process in the host, while maintaining functionality, be it degradation or long term structural integrity [58]. Therefore, during cell culture in vitro or implantation in vivo, scaffolds must either degrade, releasing non-toxic bi-products, or become incorporated into the tissue construct by mechanisms of remodeling [58]. Although some basic criteria have been outlined for scaffold-based tissue engineering, the requirements for scaffold design are largely tissue or organ and cell specific.

Scaffolds for tissue engineering small caliber vascular grafts should provide both mechanical and functional support similar to the ECM until the cells are stabilized by the production of their own ECM. The scaffold shape must be cylindrical, hollow and of a reasonable diameter $(<6 \mathrm{~mm})$. The scaffold micro-architecture must also mimic the specific tissue for which the final construct is intended to replace. In native vessels, cells interact within a three-dimensional, fibrous ECM through which matrix morphology and mechanical behavior promotes cellular development [59]. Therefore, further requirements for a successful TEVG are found in the ECM mimicking micro-structural properties of the scaffold including porosity, pore size, and general architecture (fibrous, spherical voids etc.) [60]. Bio-mimicking the 3D ECM by fabricating fibrous scaffolds with nanometer fiber diameters enhances vascular endothelial and smooth muscle cell adhesion and proliferation in scaffolds, with fiber diameters in the range of $50-500 \mathrm{~nm}[61,62]$. ECM protein fibers are 1 to 2 orders of magnitude smaller than the cells 
they interact with, allowing cells to interact with several fibers at one time [58]. The scaffold should thus maximize available surface area to create a 3D network for cell interactions.

Porosity, the percentage of pore space in a solid, is necessary for nutrient and waste exchange and cell signaling [63]. Pore size is also critical for the migration and ingrowth of cells and the transport of proteins $[63,64]$. There is however a delicate balance. If the pore sizes are too large $(>10 \mu \mathrm{m})$, cells will be unable to find enough surface area for successful attachment and if the pores are too small $(<10 \mu \mathrm{m})$, cells will be hindered from necessary migration into the scaffold. Furthermore, high porosity (> 90\%) will promote increased cell infiltration. However, there is a corresponding decrease in mechanical strength by a power-law relationship [65]. Pore interconnectivity is also important as it allows for cell migration and ECM infiltration. Generally, without pore interconnectivity, a porous scaffold would serve no purpose. An ideal scaffold would have $100 \%$ pore interconnectivity to maximize the transport of nutrients throughout the construct [59].

In summary, ideal scaffolds for TEVGs will be physiological representatives of the native blood vessel in strength and architecture, with a high surface to volume ratio to facilitate cell attachment, differentiation, communication and circulation of all necessary nutrients, waste and growth factors.

\subsubsection{Progress in TEVG Development}

TEVGs have been developed using a variety of materials, cells, and bioreactor designs. The first engineered vascular construct was developed by Weinberg and Bell who developed a tubular vessel out of bovine ECs, SMCs and fibroblasts surrounded in a Dacron sleeve for added strength [66]. Since then, both natural and synthetic materials have been used for 
TEVGs. Niklason et al. developed TEVGs using polyglycolic acid (PGA) scaffolds seeded with vascular SMCs and ECs, cultured in a pulsatile perfusion bioreactor. These tissue engineered constructs were implanted in swine with patency up to 24 days [67]. L'Heureux et al. showed the feasibility of generating a completely biological TEVG from human cells and was able to create all three layers of the blood vessel; intima, media and adventitia. Analysis of these TEVG's showed the graft had appropriate mechanical strength and a confluent endothelium was achieved, however burst pressure was much higher than the native artery [52, 54].

A variety of bioreactors have been used to stimulate culture environments such as pulsatile perfusion bioreactors, rotating vessel chambers, and continuous flow systems. Each type of bioreactor is used to stimulate a certain cellular function. To date, there is no scaffold/cell/bioreactor/stimulation system that has overcome the challenges of creating small diameter TEVGs to meet all the requirements for a successful vessel mimicking construct for implantation. Although a successful TEVG for replacement or bypass therapy has not been developed, the progress that has been made has opened up many doors to other scientific investigations [54]. One such avenue of TEVG application is to provide an in vitro model for device testing.

\subsubsection{Blood Vessel Mimic as an In Vitro Vessel Model}

In 2006, Cardinal et al. published work on the ability to use TEVGs in vitro to test the response of an intimal lining of endothelial cells to stent implantation [3]. The study suggests assessment of new stent configurations and designs in an in vitro tissue model would provide more physiological, cost effective, high throughput and convenient results of cellular-device 
interactions prior to pre-clinical animal testing. The study used human endothelial cells from adipose tissue pressure sodded on the lumen of an ePTFE scaffold. Cells and scaffold are matured in a flow bioreactor until a confluent cell lining has been developed on the inside of the ePTFE. The model consists of a rectangular vessel chamber (Figure 5), a media reservoir and a peristaltic pump. Stents were deployed into tissue lined scaffolds within the bioreactor and endothelialization was observed. Such endothelialization was consistent with what results obtained for animal studies of bare metal stent designs $[3,68]$. The study shows the feasibility of using a blood vessel mimic (BVM) with a human cell lining to quantitatively evaluate the tissue response to stents in vitro.

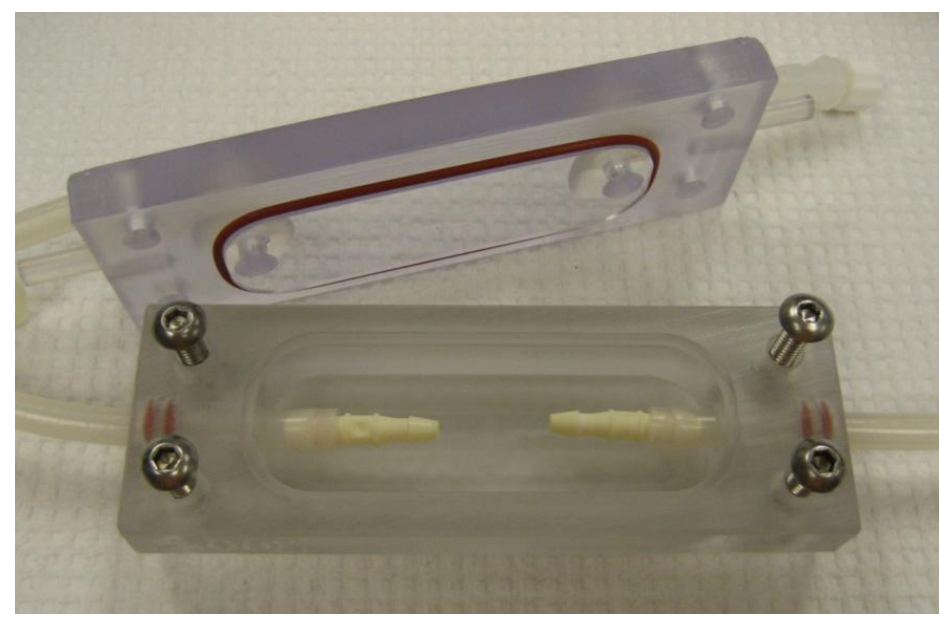

Figure 5 - The Blood vessel mimic (BVM) system above is made of a polymer casing with a lid that attaches with screws. The scaffold is pushed onto the two fittings inside the chamber and sutured in place.

One of the major advantages to the BVM design is the ability to produce a consistent, controlled model for directly comparing and evaluating several stent designs. There are however, several physiological and mechanical limitations to the BVM system at this time. The system utilizes a peristaltic pump which provides media flow but does not account for the pressures, pulsatility, and fluid shear stress experienced by the native vessel. Also, the BVM 
does not contain a media or adventitia layer and therefore smooth muscle cell and contractility responses of the vessel due to stent implantation, specifically drug eluting stents, could not be evaluated [3]. Furthermore, while the BVM system uses nutrient rich media, the use of blood could possibly give insight into the relation between blood flow and development of restenosis [3]. There are other limitations and disadvantages to be addressed including scalability and user friendliness. One major limiting factor of the BVM is the use of ePTFE. Primary disadvantages to using ePTFE are the high cost, the dependency on ePTFE suppliers, and non-native mechanical properties, as discussed previously in the Introduction of this thesis.

Currently, under the guidance of Dr. Kristen Cardinal of California Polytechnic State University, several lab researchers are working toward making a more physiological, user friendly, high-throughput and cost effective BVM system. The latest version of the BVM bioreactor is shown in Figure 6. The modified system allows for more simplicity, ease of assembly, and accommodation of varying sized scaffolds and vessel morphologies. The new BVM system utilizes a leak-proof snap and seal container for the vessel chamber. This update allows for easy access to and insertion of the scaffold in the bioreactor. New adjustable length fittings to accommodate varying length scaffolds and stents were added as well. With the new system design, introduction of pulsatility and wall shear stress to better simulate in vivo conditions of the native blood vessel are being investigated. Incorporation of SMCs, in addition to ECs, to create the media of the blood vessel is also being investigated. 


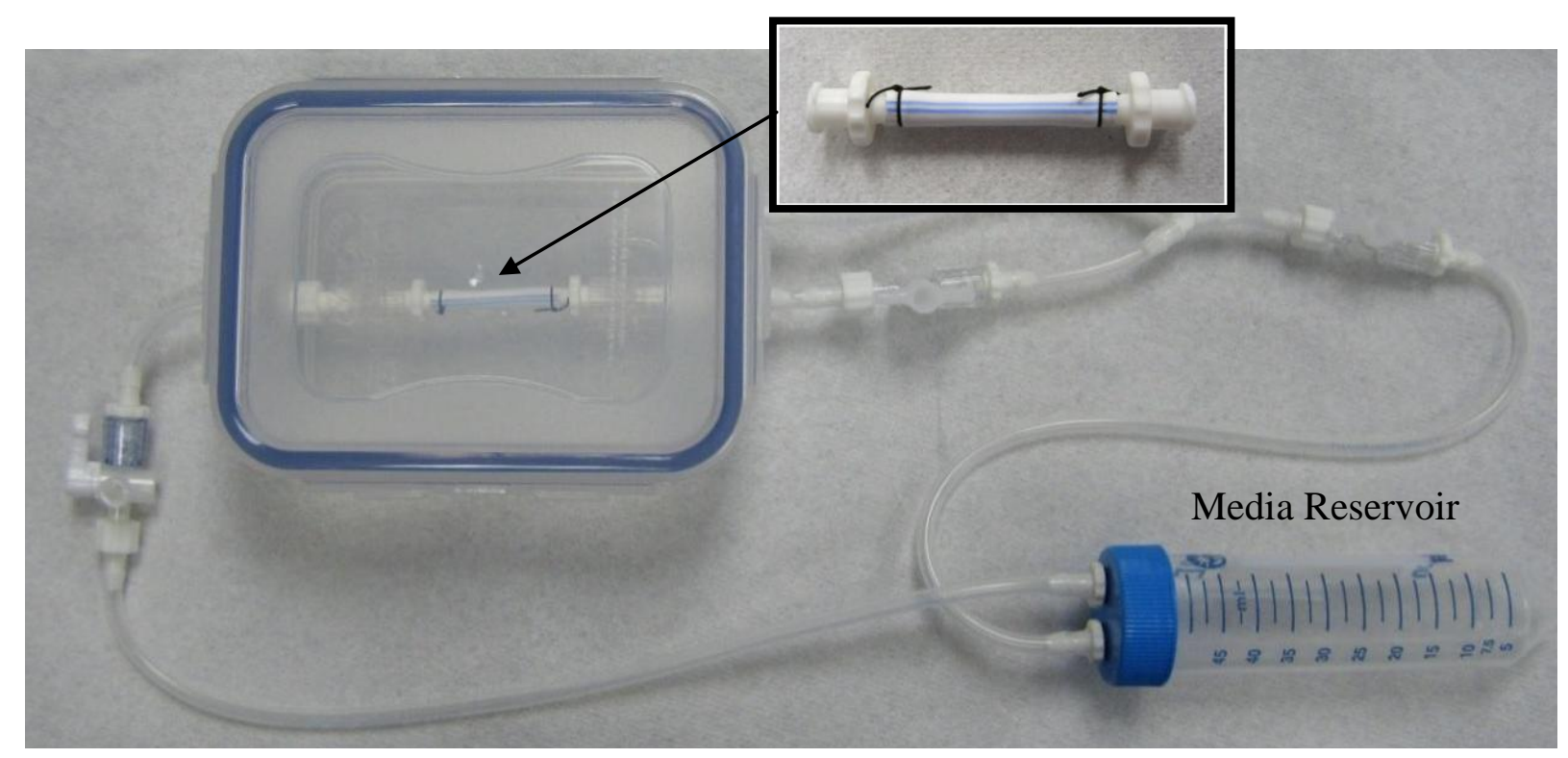

Figure 6 - The leak proof polypropylene container allows more room for scaffold insertion. The container uses adjustable fittings for different length scaffolds. The above image shows ePTFE sutured onto the fittings inside the new bioreactor chamber. The system contains a media reservoir and is can be hooked up to a pulsatile perfusion pump.

To address the limitations of ePTFE, a need exists for a scaffold that better represents the native vessel in mechanical behavior (i.e. tensile strength, elastic modulus) and architecture, which can be developed in-house, and is less expensive. The desire to mimic the native blood vessel is imperative to proper TEVG development and such mimicking behavior can be achieved through material selection and processing. Different biomaterials can be formed into different 3D morphologies by various processing techniques and can achieve a range of material properties. Some fabrication techniques used to develop porous tissue engineering scaffolds include, gas foaming [69, 70], solvent casting and particulate leaching [71, 72], fiber bonding [73], extrusion [74], solvent induced phase separations [75, 76], rapid prototyping [44] and freeze drying [77]. One such fabrication method known as electrospinning, although still in developmental infancy, is a relatively simple, cost effective process of routinely creating 
continuous micron to nano-sized fibers in a highly porous network similar to the ECM for tissue engineering applications $[58,78]$. Recently, electrospinning was introduced into the lab and research was conducted on the feasibility of in-house, consistent fabrication of electrospun scaffolds and results were promising. This thesis aims to expand on those results to implement electrospun scaffolds into the BVM model. For this reason, electrospinning will be reviewed and discussed in the following section.

\subsection{Electrospinning - An Overview}

Electrospinning is a fabrication technique investigated for the in-house production of polymer scaffolds as a replacement to ePTFE for the in vitro BVM system. The following sections will discuss the history and theories behind electrospinning and how the system has been utilized to develop scaffolds for TEVGs. Processing parameters of the system in relation to construct architecture and optimization of cell interaction will be discussed in detail.

\subsubsection{History of Electrospinning}

Electrospinning is the process of extruding a fine fiber from a charged polymer solution toward a grounded collector. The fiber is continuously stretched by electric static forces and the evaporation of solvent while traveling through air prior to deposition [79]. The science and understanding behind electrospinning began with electrospraying over 100 years ago, in which polymer droplets, rather than fibers, were formed by electrostatic forces. In 1934, Formhals patented the first device that successfully electrospun cellulose acetate into aligned small diameter fibers using an acetone solution [80]. Formhals continued to improve upon his work with additional patents over the next few years with minor, but effective changes, to the

electrospinning setup [81, 82]. In 1969, Taylor developed a mathematical model to describe the 
shape of a fluid droplet under an applied electric field. The characteristic shape is called the Taylor cone. Taylor determined the balance between surface tension and electrostatic forces, necessary for a successful jet formation, was achieved when the droplet cone angle was 98.6 degrees [83]. In 1971, Baumgarten investigated solution processing parameters and the corresponding effects on fiber diameter [84]. Baumgarten determined increased solution viscosity lead to increased fiber diameters and as a result was able to create electrospun fibers from 500-1,100 nm. Since 2000, the number of publications on electrospinning has increased exponentially [79]. Not until the most recent work and accomplishments has the full potential of electrospinning been realized. The ability to fabricate fibers with nano-sized diameters has become a promising reality for areas of filtration, membranes, wound dressings, drug delivery devices and tissue engineering scaffolds $[78,85]$.

Fiber diameters of electrospun constructs have been achieved between several microns to just a few nanometers [86]. The high surface area per unit volume ratio achieved in electrospun nano-fibers can allow for enhanced cell proliferation, adhesion and differentiation in culture. Promising applications of electrospinning have been seen for several engineered tissues including vasculature $[47,48,56,63,87]$, bone [41] and neural tissue [88]. The range of polymers that can be electrospun is only limited by the ability of the polymer to be brought into solution or melted. Synthetic as well as natural polymers, such as collagen [89], elastin [56] and fibrinogen [90], have been successfully electrospun. Drugs, antibodies, enzymes and natural/synthetic material complexes have also been incorporated into the electrospun constructs by including the substances in the polymer solution or by co-spinning [78, 91, 92]. Additionally, electrospun nano-fibers can be organized into a variety of architectures by manipulating 
alignment and stacking of the fibers through various electrospinning protocols and setup modifications [93].

\subsubsection{Electrospinning Process}

Although simple to employ, the electrospinning process is a fairly complex system with multiple parameters that can be varied for construct and fiber optimization for a given application. The general electrospinning setup consists of a charged polymer solution or melt, a capillary though which the polymer is forced, a pump, a grounded collector or target, and a high voltage supply (Figure 7).

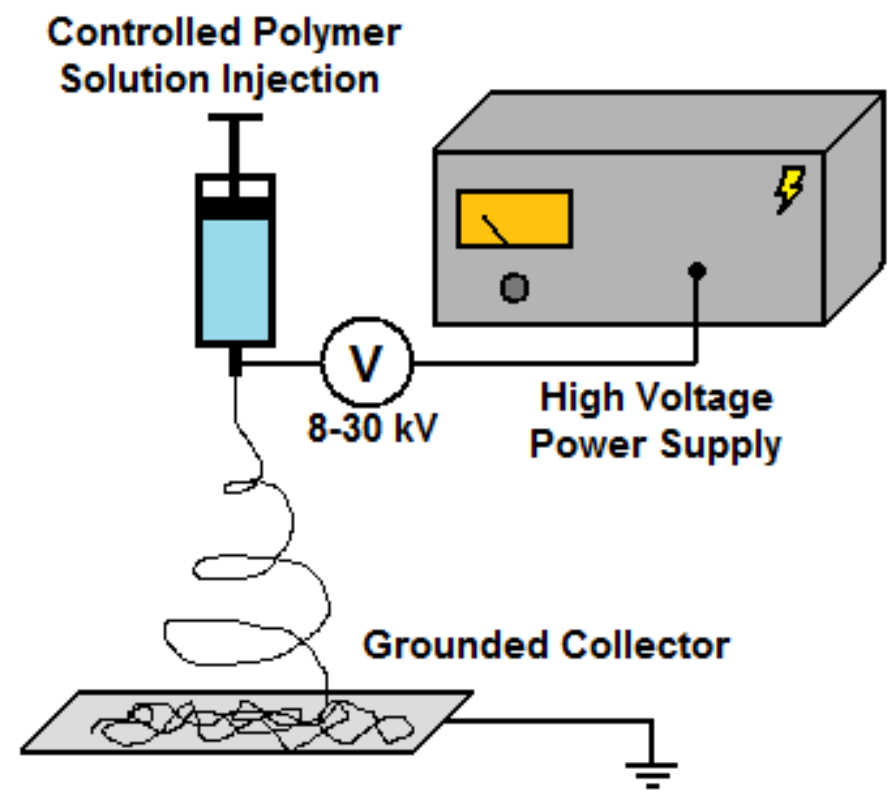

Figure 7 - A schematic representation of a typical electrospinning setup. The setup consists of a high-voltage power supply, a polymer solution and a grounded collector.

The polymer solution is composed of a dissolved polymer in a volatile solvent. The role of the solvent is to adequately bring the polymer resin into solution and evaporate quickly when 
exposed to air. Evaporation of the solvent is necessary for the production of a mostly solid fiber upon collection. The pump holds a reservoir, generally a syringe, containing the polymer solution and is responsible for regulating the flow rate of the solution. The high voltage source generally outputs voltage from $8-30 \mathrm{kV}$. An electrode from a high voltage source is applied to the solution usually by placing the electrode directly on the metal capillary or syringe needle. Depending on the application, various collectors can be implemented to acquire different shaped scaffolds such as a wire mesh, a flat plate, a fluid bath or a rotating plate around which the polymer wraps itself [56]. Stationary collectors are used to achieve randomly oriented fiber meshes where as rotating collectors can achieve varying degrees of fiber alignment depending on the speed at which the collector rotates. For the purpose of tissue engineering blood vessels, a mandrel or rod which both rotates and translates back and forth can be used to fabricate tubular scaffolds of a specified length, uniform thickness, as well as diameter. The distance, perpendicular to the collector, between the needle tip and the ground, is called the gap distance. The separation between the charged polymer solution and the grounded collector creates a static electric field.

When a high voltage is applied to the metal capillary, the fluid droplet at the tip experiences both electrostatic repulsion between surface charges and Columbic forces exerted by the electric field [79]. As the charge increases, the droplet changes from a spherical shape to the Taylor cone. As the charge separation between the solution and the collector overcomes the surface tension of the droplet, a thin polymer jet accelerates from the Taylor cone towards the collector. A whipping instability transpires as the jet travels through the air, causing the jet to progress in a zigzag or conical pattern, which further thins the fiber diameter as the solvent evaporates before final deposition on the collector. An extensive mathematical model of this 
dynamic jet instability has explained the whipping instability to be a result of repulsive interactions between the jet and the external electric field [94].

\subsubsection{Optimization of Processing Parameters}

Several electrospinning processing parameters can be adjusted and possibly optimized in order to fabricate ECM mimicking scaffolds for TEVG development. Parameters include solution parameters (e.g. viscosity, conductivity, solvent volatility etc.), operational parameters

(e.g. gap distance, flow rate, electrical field strength) and environment (e.g. temperature, humidity etc.) $[91,95]$. However, success is not limited to the alteration of a single parameter, but often a combination of several dependently operating parameters will be necessary to achieve continuous, small diameter fiber morphologies. Several studies have been conducted on the interconnectivity of processing parameters and construct morphology. The following subsections will outline the major known contributors to electrospinning construct endpoints and variations of morphological characteristics, including the effect those construct outcomes have on cellular function for tissue engineering vascular grafts.

\subsubsection{Applied Voltage}

Small changes in the applied voltage can lead to large changes in fiber morphology. Applied voltage will initiate as well as destroy polymer jet formations. Deitzel et al. studied the effects of voltage on fiber morphology and bead defects in electrospun polyethylene oxide (PEO)/water solutions (bead defects are essentially globs of polymer that form along the length of the fibers). The study determined that increasing voltage changed the shape of the Taylor cone at the tip of the capillary, which in turn increased jet instability and the formation of bead defects. Increasing the voltage even further led to the disappearance of the droplet at the 
capillary tip and the jet would then be pulled from within the capillary [85]. In other words, the electric field strength is removing the polymer from the reservoir faster than the pump is feeding polymer into the capillary, thus eliminating the droplet and Taylor cone formation necessary for an electrospun construct. This was confirmed by Zong et al. [96]. Another study on the effects of applied voltage on droplet and jet formation confirmed jet stability occurs when the average flow rate to the tip of the needle was matched by an equivalent flow rate at which the fluid was carried away by the jet [91]. The same study showed that when the voltage was too low, the droplet size increased and the gravitational forces overcame the field and pulled the droplet to the floor [91]. Buchko et al. showed an increase in applied voltage not only increases the field strength, creating smaller fiber diameters due to greater electrostatic stresses, but also draws more polymer solution from the syringe tip or capillary [97]. Consistent with Buchko, another group also noticed a decrease in fiber diameter as a result of increased applied voltage for a 5 wt\% solution of poly(L-lactide -co-e-caprolactone) $[\mathrm{P}(\mathrm{LLA}-\mathrm{CL})]$ with all other variables constant [98]. In summary, a high voltage supply $(1-30 \mathrm{kV})$ is necessary to overcome the surface tension of the polymer solution, thus initiating the formation of a jet into a single continuous fiber. Moreover, electric field strength contributes to the final fiber diameter of an electrospun construct, but must nevertheless be in balance with the feed rate of the solution. However, studies have shown that applied voltage has a less significant effect on fiber diameter than solution viscosity $[91,98]$.

\subsubsection{Solution Viscosity}

There is a significant correlation between solution viscosity and fiber diameter; fiber diameter increases with increased concentration of polymer solution. Adjusting solution viscosity is likely the most effective method of altering fiber diameter during electrospinning and 
several studies have shown a linear relation between the two [85, 98-101]. Solution concentration inversely alters the surface tension of the liquid. Decreasing surface tension reduces the number of bead defects present along fibers. When increasing solution viscosity, beads become larger and more elongated while the average distance between the beads becomes greater until bead-less fibers are formed [102]. This removal of beads is subsequently accommodated by an increase in fiber diameter.

When the solution concentration and viscosity is lowered to unstable levels, the electrospinning process transitions from a fiber forming jet to electrospraying, where capillary instability is driven by a high surface tension [102]. Demir et al., determined lower and upper concentration boundaries at $3.8 \mathrm{wt} \%$ and $12.8 \mathrm{wt} \%$, respectively, for solutions of polyurethanurea in dimethylformamide (DMF). At $3.8 \mathrm{wt} \%$ solution concentration, surface tension dominated the electric field strength and electrospraying was observed. Above $12.8 \mathrm{wt} \%$ solution no jet was formed. Further analysis of solution concentrations between $3.8 \mathrm{wt} \%$ and $12.8 \mathrm{wt} \%$ showed bead defects occurring at the lower concentrations and curled fibers at higher concentrations, both of which decrease the much desired surface area to volume ratio for TEVG scaffold morphology [101]. The combination of high electric field strength with low solution concentrations can lead to multiple jet formations from the droplet at the tip of the capillary. Demir et al. observed this behavior, as a result, greater distribution of fibers formed on and around the collector [101]. Deitzel et al. studied the effects of solution concentration on fiber morphology and determined that low concentrations of solution had large variations in fiber diameters and the presence of fiber bundles, most likely a result of un-evaporated solvent upon collection. At higher concentrations, fibers showed consistent uniformity in diameter with few fiber bundles [85]. 
In summary a critical range for polymer concentration exists for the electrospinning setup to function appropriately and to achieve a desired fiber diameter. It is important to note that every polymer and solvent solution will have a different critical range due to differences in solvent and polymer characteristic properties, including; density, volatility (boiling point), viscosity, surface tension, and conductivity.

\subsubsection{Solvent Selection}

The main criteria for selecting solvents are to ensure the solvent is capable of bringing the working polymer into solution and to be volatile enough to dissolve rapidly when exposed to conventional atmospheric environments. Other roles of the solvent include controlling surface tension, electrical conductivity, and viscosity of the solution [93]. If the polymer solvent solution cannot adequately carry an electrical charge, ionic surfactants can be incorporated into the solution. The use of cationic surfactants, such as tetrabutylammonium (TBAC) or dodecyltrimethylammonium bromide (DTAB), has been shown to successfully increase the conductivity of the solution leading to the creation of continuous small fiber diameters [93, 103]. Also, one study has demonstrated increased volatility leads to increased porosity and surface area in electrospun constructs compared to lesser volatile solvents [104].

\subsubsection{Flow Rate}

Fiber diameter increases with increasing flow rate and bead formations occur when the flow rate is too high [78]. The importance of flow rate, as depicted by Taylor, is to force polymer solution through the capillary at the same rate the solution is being carried away. Such balance between flow-in and flow-out is necessary to maintain continuous, unbroken fiber formations [83]. If the flow rate is too high, the polymer solution will be forced out faster than 
the jet can carry it away, increasing the droplet size at the tip of the capillary to unstable proportions and initiating bead formations along the length of the fibers. Eventually as the droplet grows larger, gravity over takes the droplet and brings it to the ground. Consequently the once continuous fiber will be broken and a new fiber will begin.

\subsubsection{Gap Distance}

The distance between the capillary or needle tip and the grounded collector is called the gap distance. Gap distance is known to affect the outcome of fiber size however, these effects are minimal. Fiber diameters decrease with increasing distance from the Taylor cone by 1-2 orders of magnitude $[78,105]$. As mentioned previously, as the fluid jet travels towards the collector, the jet undergoes a whipping instability. This instability is necessary for the thinning of jet fiber diameter and the evaporation of solvent. When the gap distance is too short, there is inadequate distance and time for the solvent to evaporate and the polymer will collect on the target in a wet state [89]. Megelski et al. noticed the aforementioned trend when studying electrospun polystyrene fibers and noticed increased bead formations with decreased gap distance as a result of residual solvent in the collected polymer [104]. Consequently the increase in gap distance allows for adequate time for solvent evaporation and increases the traveling distance for whipping instability to further thin the fiber. There is, however, a critical distance at which the gap is too large and the electric field strength will no longer be able to overcome the surface tension of the liquid and the polymer will not collect on the target.

\subsubsection{Needle Size}

The effect of needle size on fiber morphology during electrospinning has been minimally investigated. The basic function of the needle is to support an electrode to charge the polymer 
solution. The needle also acts as a narrow exit point for the solution to be drawn through. The main requirement of the needle is to not prohibit solution flow. Mo et al. electrospun P(LLACL) using 18G, 21G, 22G, and 27G needles. Clogging and beading was observed with all needle sizes except the $27 \mathrm{G}$ needle $(0.4 \mathrm{~mm}$ diameter). However, the researchers argued that bead free fibers and no clogging could be easily obtained through increasing the solution concentration for larger diameter needles [98]. There is however, no conclusive study that suggests a smaller needle size can improve fiber quality or decrease fiber diameters. There is no apparent gold standard for needle size in electrospun constructs from primary literature. 


\subsubsection{Fiber Diameters and Porosity}

Bio-mimicking the fibrous ECM to produce nanometer fiber diameters enhances vascular endothelial and smooth muscle cell adhesion and proliferation in scaffolds, with fiber diameters in the range of 50-500 $\mathrm{nm}[61,62,106]$. These small fiber diameters allow for cells to contact multiple surfaces at one time. The hypothesis has been proposed that proper cell phenotype will not be adequately achieved if the tissue engineered scaffold has fiber diameters equal to or greater than the cell diameters [107]. The main electrospinning operational parameters affecting fiber diameter are solution viscosity, applied voltage and the solution flow rate. Generally, fiber diameters increase with increasing viscosity, decreasing electric field strength and increasing flow rate.

Control of pore size and porosity is an attractive feature of electrospinning and is critical to proper cellular adhesion, proliferation, differentiation, and nutrient transport [60, 63-65]. The suggested optimal pore size for cellular infiltration is a minimum of $10 \mu \mathrm{m}$ [108]. Much of the excitement over electrospinning has focused on the ability to produce nano-fibers, with little attention to porosity. Although the nano-fibrous structure produced by electrospinning closely mimics the native ECM, the spatial characteristics associated with nano-fibers and the subsequent porosity do not reflect that of the ECM $[95,109]$. Eichhorn et al. presented a theoretical, mathematical model to conclude that fiber diameter is directly related to pore size in electrospun constructs with cell penetration increasing proportionally with increasing fiber diameter [110]. Since pore size decreases with decreasing fiber diameters, what appears to be ideal fibers may lead to inadequate cellular infiltration for a 3D tissue engineered construct, due to pore sizes smaller than that of the cells they interact with [87]. Balguid et al. systematically 
investigated the optimization of cell penetration by tailoring fiber diameters of electrospun poly( $\varepsilon$-caprolactone) (PCL) with fiber diameters ranging from 3.4-12.1 $\mu \mathrm{m}$ by adjusting solution concentration, applied voltage and flow rate. Scaffolds were seeded with human venous

myofibroblasts for 4 weeks. A gradual increase in cell infiltration was observed with increasing fiber diameter and unobstructed penetration was only observed in the largest fiber diameter scaffold $(12.1 \mu \mathrm{m})$. PCL constructs showed homogenous tissue formation thus concluding the importance of fiber diameters in cellular delivery in electrospun scaffolds [87]. The study was in agreement with Eichhorns theoretical model that nano-fiber scaffolds are only beneficial as long as they are cell specific [87]. Others have shown the same results [111]. The concern associated with cellular infiltration does not necessarily apply to the BVM system. The BVM requires only an endothelium to test the response of an intimal lining to stent implantation. Therefore, nanofibers, which can increase cellular adhesion, may still be the optimum choice for the BVM.

\subsubsection{Fiber Alignment}

Fiber alignment can be used to increase radial compliance of a TEVGs [58]. Fiber alignment has been attributed to the rotation speed and translation of the rotating mandrel with random fibrous mats produced near $100 \mathrm{RPM}$ and aligned fibrous mats near or at $1000 \mathrm{RPM}$ $[100,112]$. According to Choi et al, the rotation speed of the mandrel does not influence fiber diameter [113]. However, Choi et al. demonstrated fiber orientation induced a corresponding cellular orientation but had no affect on phenotypic expression of the cells. The effects of fiber alignment on mechanical properties such as elastic modulus and strain to failure were evaluated and significant differences were found between aligned, random and orthogonal fiber orientations with highest strengths achieved in longitudinally aligned fiber constructs [100]. Aligned nano-fibrous electrospun P(LLA-CL) vascular scaffolds showed improved vascular 
SMC adhesion, parallel organization and contractile phenotype compared to unaligned polymer constructs [114]. These aligned scaffolds also achieved tensile strengths similar to the native artery therefore suggesting aligned constructs could be ideal for TEVGs. However, it seems no current preference as to fiber orientation given the successful tissue formations seen in both randomly oriented and aligned fibrous scaffolds [115].

\subsubsection{Environment}

A well controlled environment may be required to maintain a reproducible operation and such factors as humidity may play an important role in construct morphology [104]. At this time, there is little to no published work on the effects of humidity and temperature on the electrospinning of TEVGs.

In summary, electrospinning offers several advantages to scaffold development for tissue engineering vascular constructs. The flexibility in set-up design and the ability to tailor fiber morphologies and porosity through processing parameter optimization allows for tissue and cell specific scaffold development. Electrospinning offers a probable alternative to purchasing

ePTFE scaffolds for the BVM design, potentially allowing for in-house scaffold production that is scalable, controlled, and economical.

\subsection{Summary and Aims of the Thesis}

Coronary artery disease is affecting thousands of Americans each day. Advancements in stent designs and treatment modalities necessitate better preclinical testing with fast reliable results. The feasibility of an in vitro blood vessel mimic (BVM), that employs tissue engineered blood vessels for intravascular device testing, has been demonstrated for bare metal stents. The 
current BVM model has several physiological and mechanical limitations, as well as scale up limitations. Improving upon the current design could have a substantial impact on intravascular device evaluation. Presently, the model utilizes the scaffolding material ePTFE, a biocompatible, non-degradable polymer commonly used in bypass grafting. Disadvantages to using ePTFE are high cost, the dependency on ePTFE suppliers, and lack of appropriate mechanical properties. An ideal tissue engineered scaffold should mimic the ECM in structure (porosity, pore size, etc.) and mechanical properties (elastic modulus, tensile strength, etc.) and be capable of supporting cell adhesion, differentiation, proliferation, communication and the transport of all nutrients and growth factors.

The aim of this thesis is to determine an alternative scaffolding material that can be electrospun to improve upon the BVM model by eliminating some of the current scaffolding limitations. In this investigation, fibrous polymer scaffolds produced in-house by electrospinning for implementation into the BVM model, were studied. The application of electrospinning to the production of tissue engineered grafts is highly favorable due to the ability to create polymer scaffolds with micron to nano-sized fibers and high porosity, both of which have shown to induce endothelial cell proliferation and attachment [78]. This thesis is driven by the hypothesis that electrospun polymer scaffolds for tissue engineering blood vessels are able to provide the framework for endothelialization and subsequent intimal lining formation in vitro and will improve upon the scalability and cost effectiveness of the BVM model. There were 3 phases to investigating this hypothesis. The initial phase was to determine a material appropriate for electrospinning scaffolds for our tissue engineered vessel constructs based on primary literature review and requirements of the BVM model. The second phase was to determine and optimize a protocol for electrospinning the new material by evaluating different solvents, 
solution concentrations, flow rates and applied voltages. The last phase of this investigation was to evaluate endothelialized electrospun constructs cultured in a flow bioreactor to assess intimal cellular lining formation. Cellular responses were evaluated using fluorescent microscopy, histology and SEM. 


\section{Chapter 2 - Polymer Selection}

\subsection{Introduction}

In 1937, DuPont patented poly(tetrafluoroethylene) [PTFE] as Teflon and considered the material to be a suitable electrical insulator. The first medical application of PTFE began almost 30 years later for an artificial heart valve [116]. Then, in 1969, W.L Gore \& Associates used a process of melting and extruding to produce a micro-porous non-woven version of PTFE, known as ePTFE or expanded poly(tetrafluoroethylene) (Figure 8). The process begins with a pure PTFE powder mixed with a volatile lubricating agent, which is then formed into a sheet. That sheet is then heated and expanded until the desired structure is reached at which point the microstructure is stabilized by an amorphous locking step [117]. The microstructure of ePTFE is characterized by solid nodes connected by thin fibers with an average internodal distance of 30 $\mu \mathrm{m}$. Porosity is near $85 \%$, but can vary based on processing parameters $[116,117]$. Early studies of ePTFE for vascular grafts showed the micro-porous nature of the graft supported better tissue adhesion [116]. The micro-porous structure and electronegative surface are critical aspects in ePTFE's ability to promote endothelialization and to minimize reaction to blood components, respectively. Today, ePTFE is the gold standard for large diameter vascular prostheses in clinical practice. However, ePTFE in small diameter sites has resulted in high rates of thrombosis within a few months in vivo $[50,118,119]$. Primarily, the cause of small caliber ePTFE graft failure is compliance mismatch leading to intimal hyperplasia. 


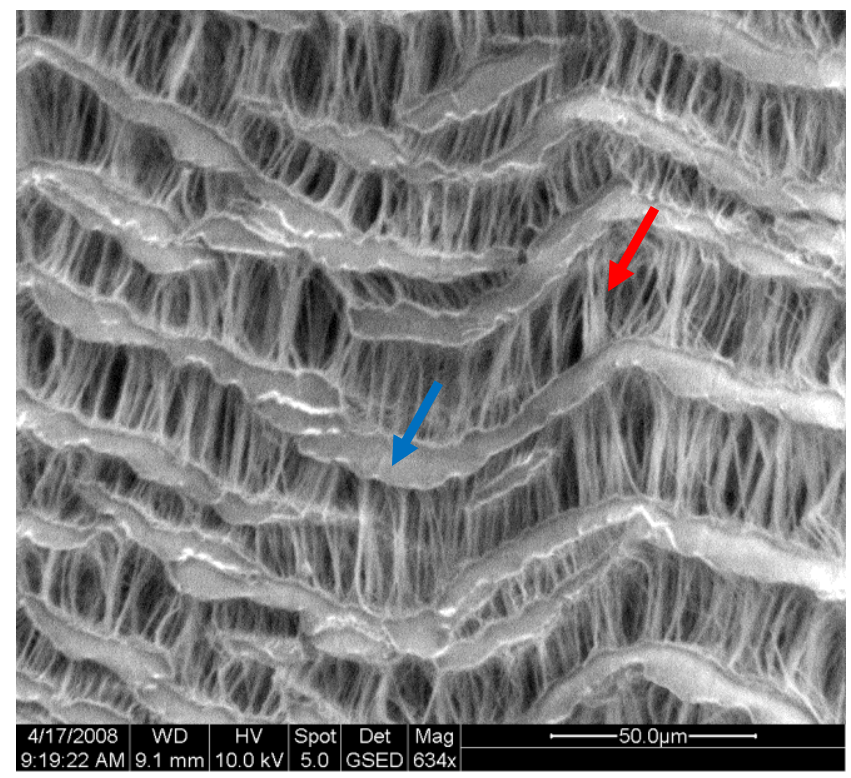

Figure 8 - The scanning electron microscope image of ePTFE (634X) shows the fibril-nodal microstructure characteristic of the polymer. The nodes (blue arrow) run parallel to the length of the tubing and perpendicular to the internodal fibers (red arrow).

As mentioned previously, the goal of the BVM model is to develop a high-throughput, cost effective, reliable model that simulates in vivo conditions in vitro to test intravascular devices prior to animal testing. The model uses tissue engineering techniques to create an intimal lining of endothelial cells on the lumen of an ePTFE scaffold. Some advantages to using ePTFE for the BVM design are the extensive pre-clinical and clinical data on endothelialization of ePTFE, ability to autoclave or gas sterilize the material, good biocompatibility, nonbiodegradability, availability in the proper size and dimensions, and proper EC orientation encouraged through its unique microstructural characteristics. However, the high cost, dependency on suppliers, and non-native mechanical properties of ePTFE, as well as the inability to tailor properties specifically for the BVM, suggests in-house fabrication of an alternative scaffold could improve upon the scalability and cost effectiveness of the BVM model. Also, a material that has mechanical properties more similar to the native vessel would create a more 
physiologically representative environment to improve EC response during culture in vitro, and the resulting responses due to device implantation. Finally, producing scaffold tubing in-house will open up further areas of research into optimizing scaffold design for tissue engineering vascular constructs.

Recently, electrospinning was introduced to the Cal Poly BVM lab and research was conducted on the feasibility of in-house, consistent fabrication of electrospun scaffolds. Studies primarily used P(LLA-CL) to assess the system, and results were promising. Those efforts were focused on manufacturing and implementing an electrospinning apparatus, and testing the ability to produce scaffolds with consistent morphologies and mechanical properties using the new technique. However, the study did not investigate electrospun constructs specifically for the BVM model. As an extension of those efforts, this thesis aims to develop electrospun polymer scaffolds for implementation into the BVM as a replacement material for ePTFE. In order to pursue such a hypothesis, the first objective of this thesis was to determine a suitable polymer for electrospinning tubular scaffolds for the tissue engineered BVM constructs.

\subsection{Methods for Material Selection}

Material selection of a polymer suitable for electrospinning BVM tubular scaffolds was conducted based on review of primary literature. Key words used for literature search were the following: electrospinning, tissue engineering, vascular grafts, and blood vessels. A table was created outlining the following criteria for each investigation: polymer name, polymer description, polymer source or vendor, application, solvents used, solvent vendor, cells and source, cell supplier, cultivation method, sterilization method, advantages, disadvantages, results, and citation. Preliminary material requirements for tissue engineering that guided material 
selection were biocompatibility and biodegradability. Both biodegradable and nonbiodegradable materials were considered as long as degradation rates could be controlled so that integrity of the construct would be maintained throughout the course of device testing. Specifically for the BVM model, the polymer cost must be reasonable ( $\$ 10 / \mathrm{cm}$ of ePTFE) and in-house sterilization must be possible. Only polymers that have been electrospun previously were considered.

\subsection{Results}

After extensive literature review, poly(DL-lactide-co-glycolide) [PLGA; 75:25; lactide:glycolide] was selected for the investigation of electrospun scaffolds for implementation into the BVM model. The following tables outline information gathered during the examination of appropriate polymers for tissue engineering that have been electrospun for biomedical applications (I, Table II). The table categories were selected based on the information that would be beneficial to material selection, as outlined in the methods section. Name, source and vendors of polymers, solvents, and cells were included to aid future purchasing if necessary. A description of electrospun construct application and the disadvantages and advantages of each investigation along with key results specifically related to the material were reported. Key factors of interest were material properties and cellular responses to the electrospun constructs. The BVM model currently utilizes human umbilical vein ECs (HUVECs) for tissue development. Therefore, evidence of appropriate HUVEC responses is desirable for material selection. Mechanical properties similar to the native vessel, if achieved, were noted given that a more compliant material is desired compared to ePTFE. Sterilization and cultivation methods were included to compare with available methods used for the BVM model. Autoclaving and ethylene oxide (EtO) gas sterilization are both available at Cal Poly for use. However, 
autoclaving is more desirable since EtO is more expensive. Lastly, cultivation methods or bioreactor conditions were included to compare responses to flow, pressure and shear stress. The ideal BVM model will simulate physiological pulsatile flow and pressure conditions, with the expectations that such conditions will lead to appropriate cellular responses during cultivation and device implantation. Although such conditions may provide appropriate physiological conditions, pressure and shear stress due to flow could have a damaging effect on the scaffold material. Therefore materials investigated under physiological flow conditions resulting in good cellular responses are highly desirable.

It is important to note that the literature review was not limited to investigations presented in the following tables, but the tables offer a summary of how electrospinning has been implemented with several different polymers both natural and synthetic for different applications. Several abbreviations are used in the following tables. Please see Appendix A for definitions of all abbreviations. 
Table I - Summary Table of Literature Review on Electrospinning Polymer Constructs for Tissue Engineering Part I

\begin{tabular}{|c|c|c|c|c|c|c|c|}
\hline 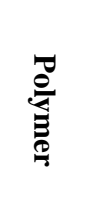 & 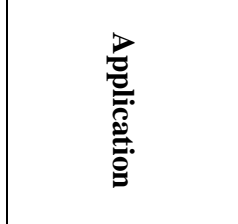 & $\begin{array}{l}\mathscr{0} \\
\frac{0}{4} \\
\varrho \\
\Xi\end{array}$ & $\stackrel{\varrho}{\bar{E}}$ & 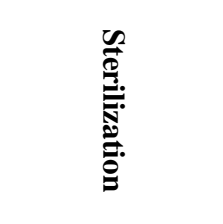 & 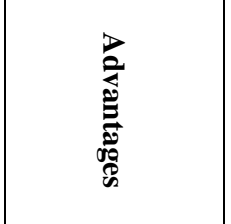 & 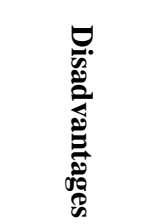 & 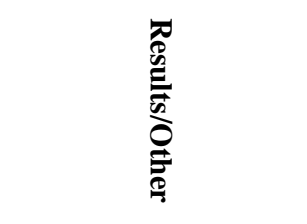 \\
\hline 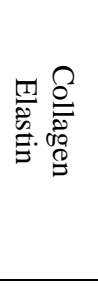 & 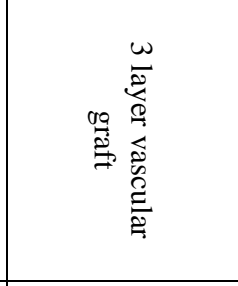 & 壱 & 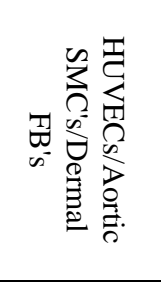 & 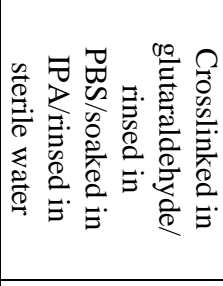 & 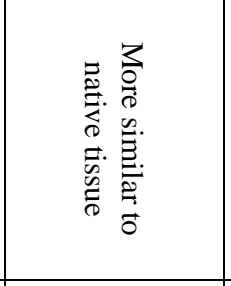 & 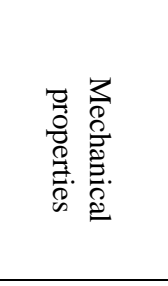 & 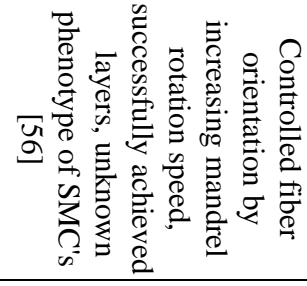 \\
\hline 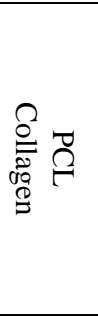 & 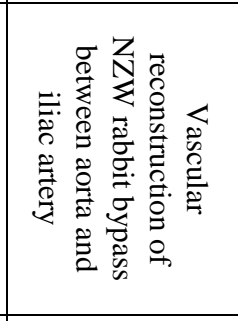 & 茎 & 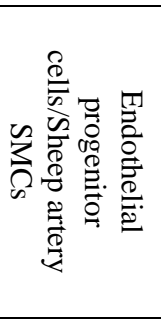 & $\frac{\pi}{0}$ & 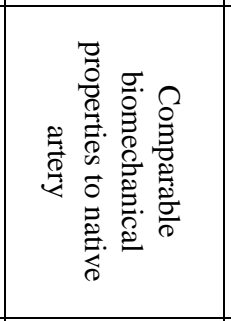 & 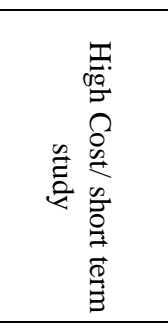 & 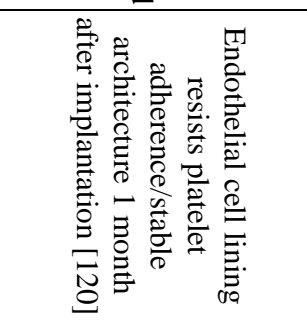 \\
\hline$\vec{\rho}$ & 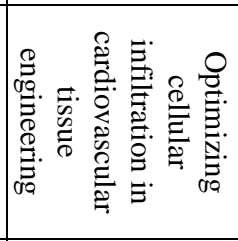 & $\stackrel{\Omega}{\Omega}$ & 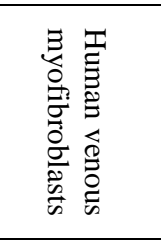 & $\Xi_{n}$ & $\Xi_{n}^{Z_{n}}$ & $\underbrace{\nabla}_{\sim}$ & 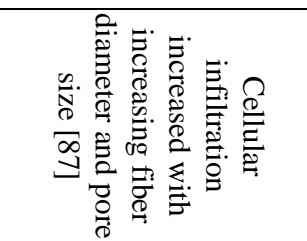 \\
\hline$\vec{\rho}$ & 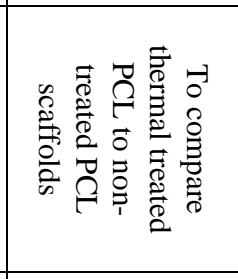 & 奇 & 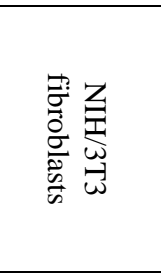 & $\frac{\pi}{0}$ & 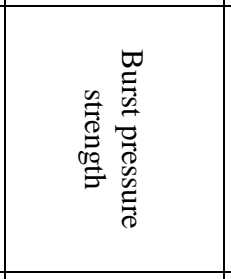 & 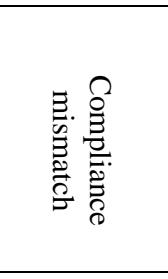 & 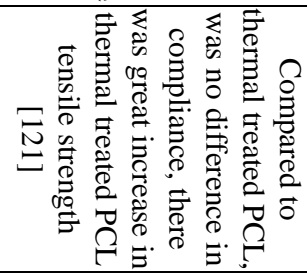 \\
\hline$\stackrel{\vec{\sigma}}{\rho}$ & 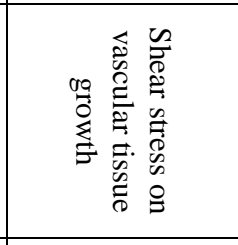 & $\underset{\mathrm{J}}{\mathrm{I}}$ & 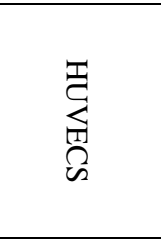 & 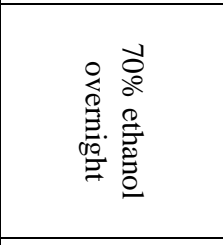 & $\underset{\sim}{\Xi}$ & $\underset{Z}{\Xi}$ & 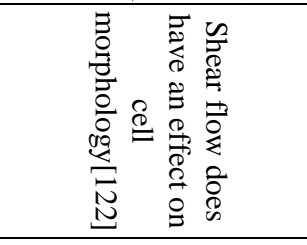 \\
\hline $\begin{array}{l}\vec{Q} \\
\vec{D}\end{array}$ & 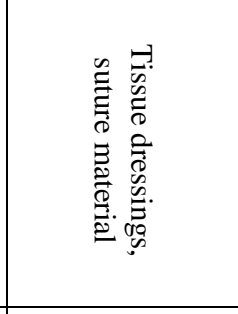 & 超 & 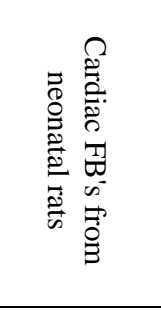 & 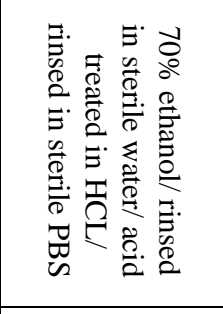 & 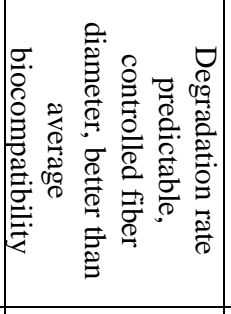 & 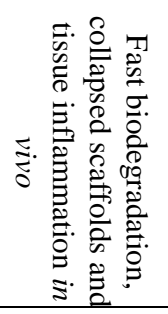 & 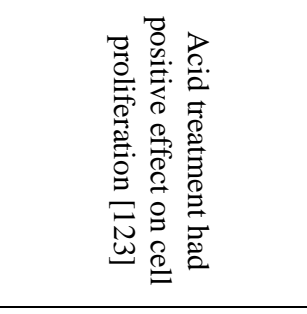 \\
\hline$\frac{\widetilde{\Omega}}{\vec{c}}$ & 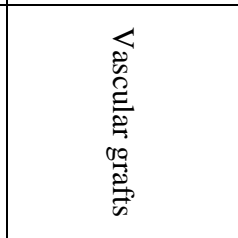 & $\underset{3}{\square}$ & 売 & $\underset{\sim}{\Xi}$ & 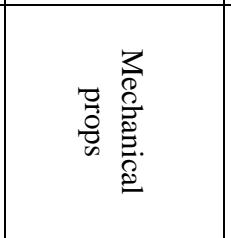 & $\underset{Z}{Z}$ & 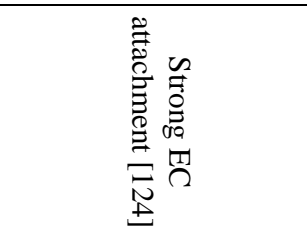 \\
\hline
\end{tabular}


Table I - Summary Table of Literature Review on Electrospinning Polymer Constructs for Tissue Engineering Part I

\begin{tabular}{|c|c|c|c|c|c|c|c|}
\hline$\frac{\overline{0}}{\grave{3}}$ & 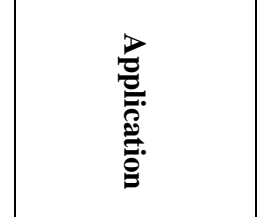 & 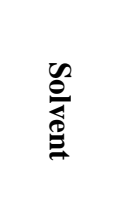 & $\frac{\Omega}{\bar{n}}$ & 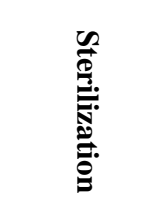 & 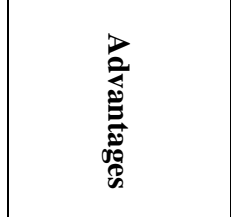 & 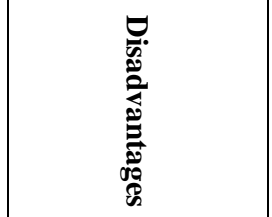 & 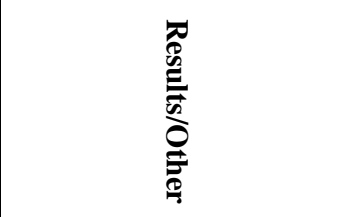 \\
\hline $\overrightarrow{0}$ & 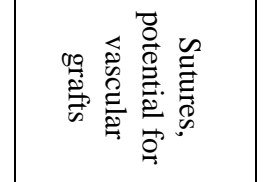 & 夏 & $\underset{\supset}{Z}$ & $\underset{D}{Z}$ & 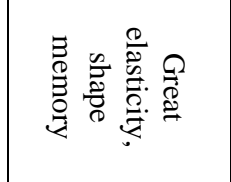 & 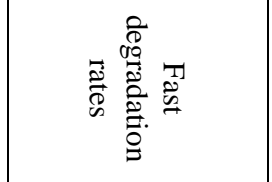 & 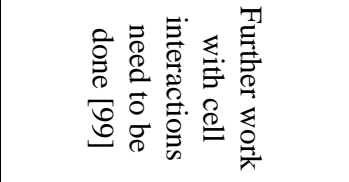 \\
\hline$\vec{C}$ & 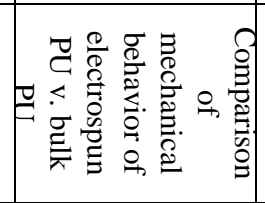 & 龸 & $\underset{>}{Z}$ & $\underset{D}{Z}$ & 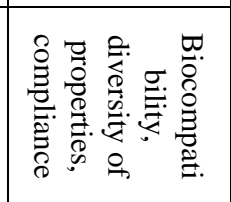 & $\underset{n}{\Xi}$ & 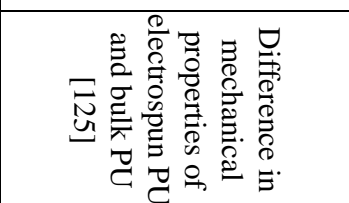 \\
\hline 茑 & 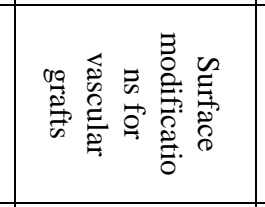 & $\vec{T}$ & 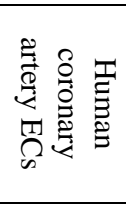 & 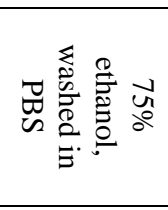 & 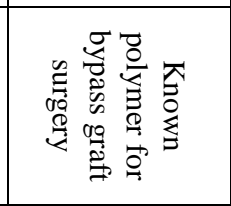 & 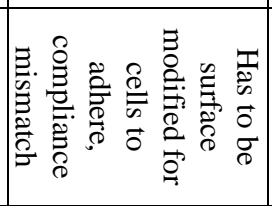 & 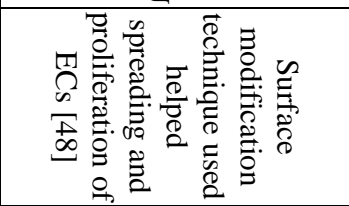 \\
\hline 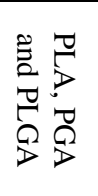 & 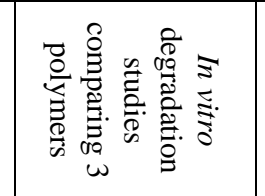 & 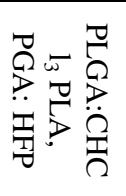 & $\underset{D}{Z}$ & $\underset{D}{Z}$ & 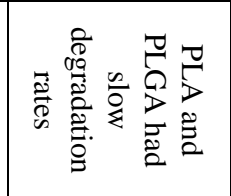 & 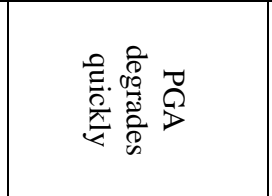 & 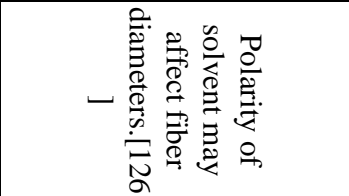 \\
\hline$\stackrel{\square}{D}$ & 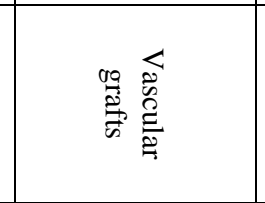 & 客青 & $\begin{array}{l}3 \\
0 \\
0 \\
0 \\
0 \\
70 \\
0 \\
0\end{array}$ & 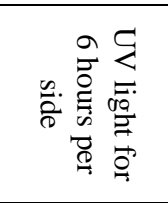 & 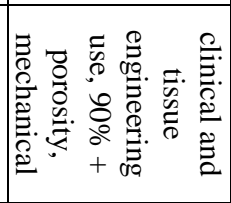 & 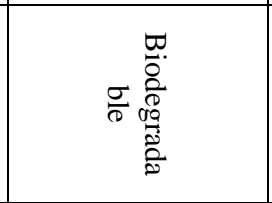 & 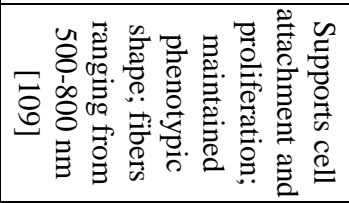 \\
\hline 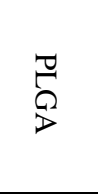 & 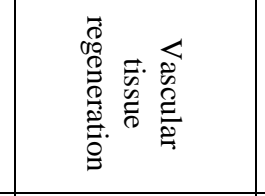 & $\stackrel{\Omega}{\Omega}$ & 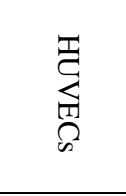 & 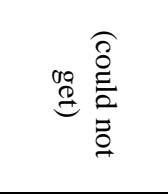 & 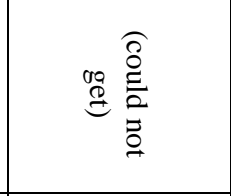 & 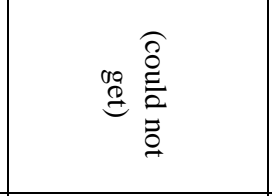 & 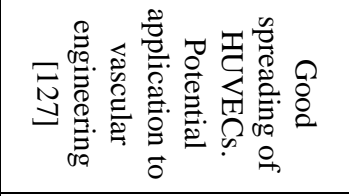 \\
\hline 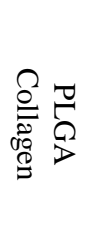 & 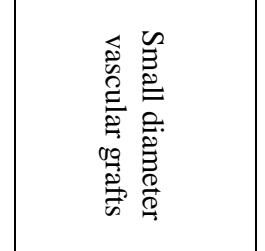 & $\Xi_{Z}$ & 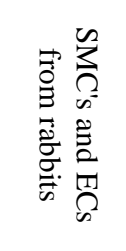 & 罟 & 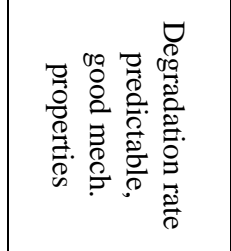 & 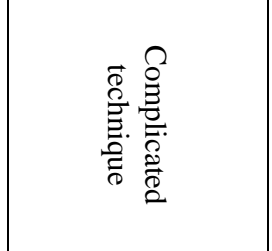 & 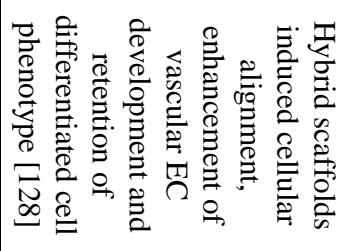 \\
\hline 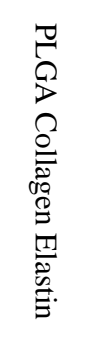 & 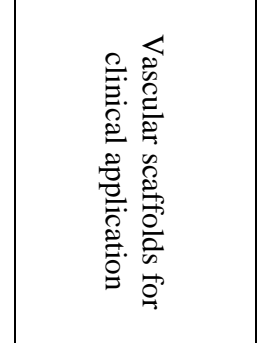 & $\underset{\mathrm{T}}{\mathrm{T}}$ & 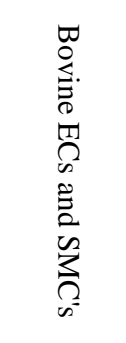 & $\underset{z}{z}$ & 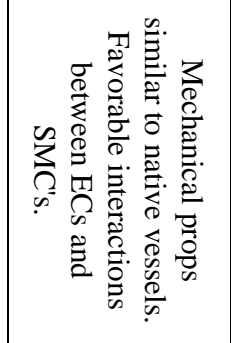 & $\mathbb{Z}_{2}$ & 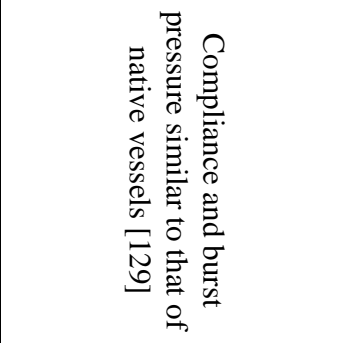 \\
\hline
\end{tabular}


Table II -Summary Table of Literature Review on Electrospinning Polymer Constructs for Tissue Engineering Part II

\begin{tabular}{|c|c|c|c|c|c|c|c|}
\hline $\begin{array}{l}\overline{0} \\
\stackrel{0}{2}\end{array}$ & 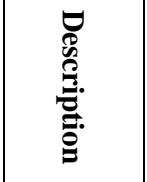 & $\begin{array}{l}\mathscr{2} \\
\stackrel{ే}{3} \\
\text { है }\end{array}$ & $\frac{\sqrt[c]{0}}{\frac{0}{4}}$ & $\begin{array}{l}\overleftrightarrow{0} \\
\stackrel{0}{0}\end{array}$ & $\frac{\Omega}{\infty}$ & $\frac{\varrho}{0}$ & 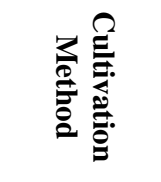 \\
\hline 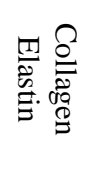 & 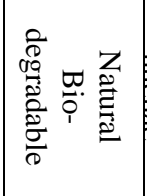 & 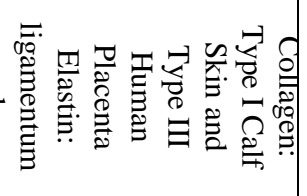 & 表 & $\frac{n}{0.0}$ & 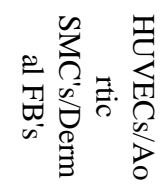 & 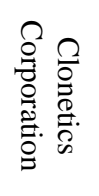 & 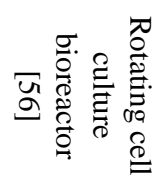 \\
\hline 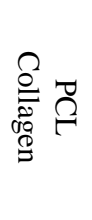 & 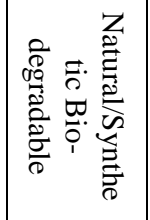 & 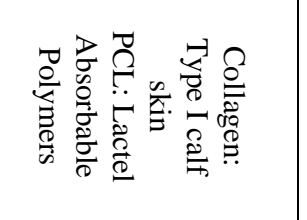 & 㞼 & $\frac{2}{0.0}$ & 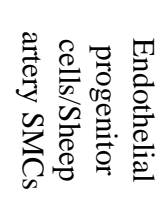 & $\underset{Z}{Z_{n}}$ & 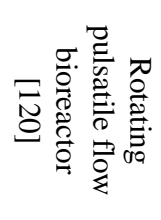 \\
\hline$\vec{\rho}$ & 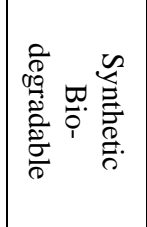 & $\nabla_{n}$ & $\stackrel{\Omega}{\Omega}$ & $\begin{array}{c}\frac{2}{0.0} \\
\frac{0}{20}\end{array}$ & 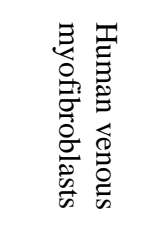 & $\nabla_{n}$ & 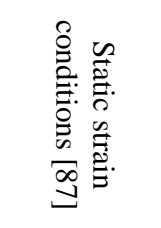 \\
\hline$\vec{\rho}$ & 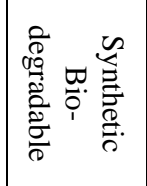 & 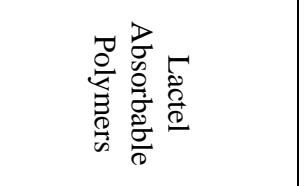 & 茎 & $\begin{array}{c}\frac{2}{00} \\
\stackrel{00}{0}\end{array}$ & 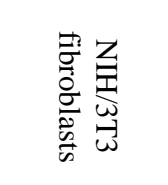 & $Z_{n}$ & 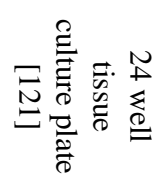 \\
\hline$\stackrel{\theta}{\rho}$ & 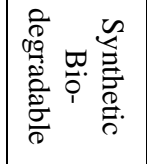 & $\underset{n}{Z}$ & 表 & $\begin{array}{c}\frac{n}{0.0} \\
\stackrel{00}{0}\end{array}$ & 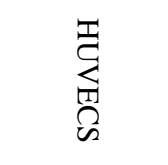 & $\frac{\lambda}{\Omega}$ & 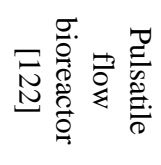 \\
\hline $\begin{array}{l}\bar{\Omega} \\
\bar{S}\end{array}$ & 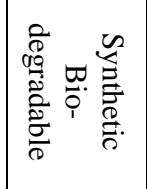 & 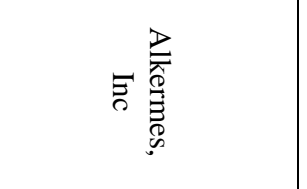 & 茎 & 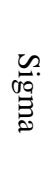 & 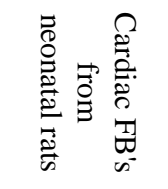 & 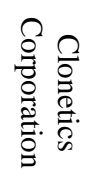 & 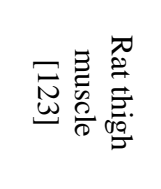 \\
\hline$\frac{\vec{\rho}}{\overparen{c}}$ & 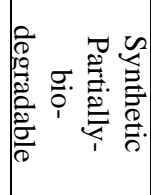 & 实产高兽兽 & $\underset{3}{3}$ & $\Xi_{z}^{Z_{n}}$ & 㞼 & 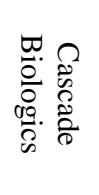 & 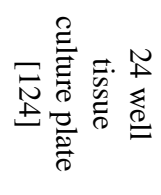 \\
\hline$\ddot{\theta}$ & 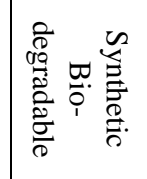 & 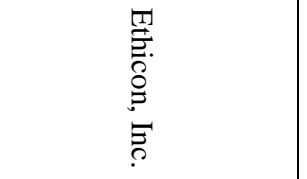 & 茎 & 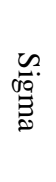 & $Z_{\supset}$ & $\underset{>}{Z}$ & $\begin{array}{l}Z \\
\frac{Z}{D} \\
\\
0\end{array}$ \\
\hline $\bar{c}$ & 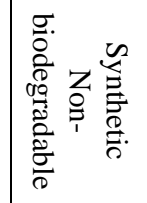 & 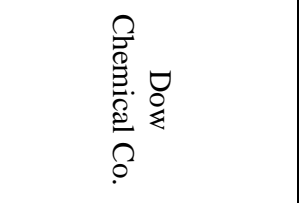 & 孛 & 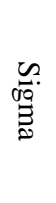 & $\underset{D}{Z}$ & $\underset{D}{Z}$ & $\begin{array}{l}Z \\
\stackrel{Z}{D} \\
\stackrel{\vec{U}}{u}\end{array}$ \\
\hline
\end{tabular}


Table II -Summary Table of Literature Review on Electrospinning Polymer Constructs for Tissue Engineering Part I

\begin{tabular}{|c|c|c|c|c|c|c|c|}
\hline 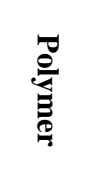 & 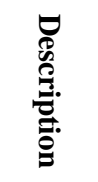 & $\begin{array}{l}\mathscr{\mathscr { O }} \\
\stackrel{8}{\mathscr{B}}\end{array}$ & $\begin{array}{l}\frac{n}{0} \\
\frac{0}{0} \\
\stackrel{0}{0}\end{array}$ & 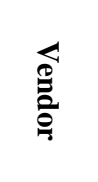 & $\frac{\Omega}{\pi}$ & $\overbrace{0}^{\circledR} \Omega$ & 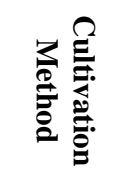 \\
\hline 㞼 & 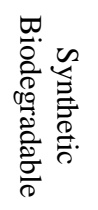 & 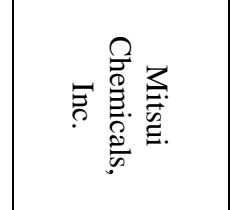 & 雱 & $\begin{array}{l}\frac{3}{9} \\
\frac{9}{2} \\
\frac{1}{n}\end{array}$ & 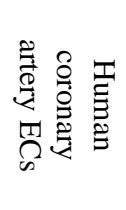 & $\begin{array}{l}\vec{D} \\
\stackrel{7}{\Omega}\end{array}$ & 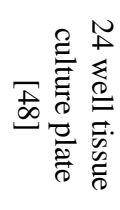 \\
\hline 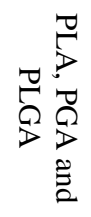 & 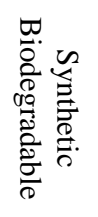 & 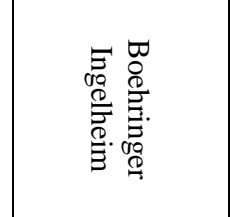 & 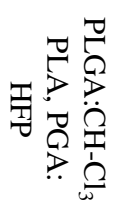 & 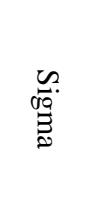 & $\underset{D}{Z}$ & $\underset{D}{Z}$ & 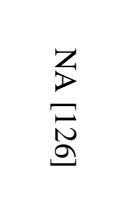 \\
\hline$\stackrel{\vec{T}}{\Omega}$ & 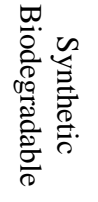 & 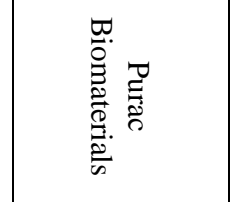 & 党勇 & 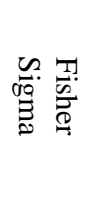 & 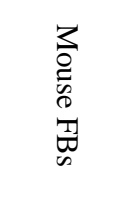 & $\Xi_{\sim}$ & 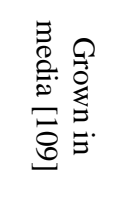 \\
\hline$\stackrel{7}{\Omega}$ & 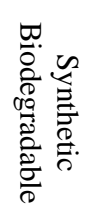 & 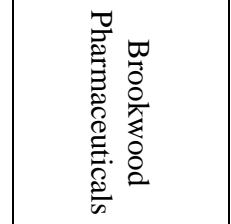 & $\stackrel{\Omega}{2}$ & 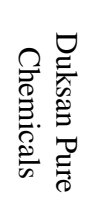 & 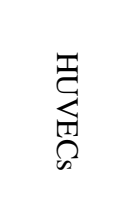 & $Z_{n}$ & 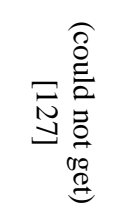 \\
\hline 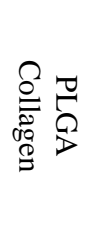 & 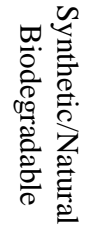 & 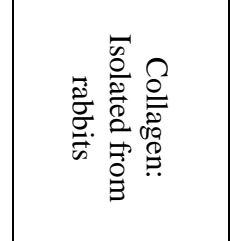 & $\overbrace{2}^{Z}$ & $\underset{Z}{Z}$ & 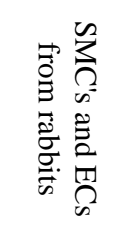 & $\underbrace{}_{\sim_{2}}$ & 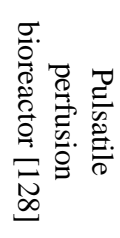 \\
\hline 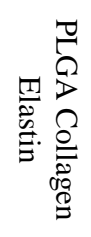 & 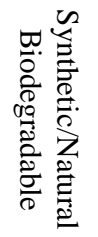 & 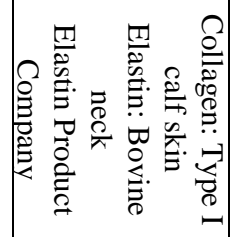 & 青 & $\stackrel{\frac{2}{00}}{\stackrel{0}{0.0}}$ & 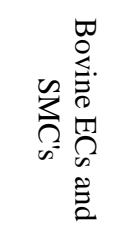 & ${ }_{z}^{Z}$ & 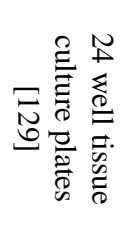 \\
\hline
\end{tabular}


Based on the resulting information gathered from primary literature, the most promising materials were poly(caprolactone) [PCL], poly(lactide-co-glycolide acid) [PLGA], polyurethane (PU), and collagen or elastin blends with PCL or PLGA. The final selection of PLGA was determined based upon EC responses, controlled degradation, acceptable sterilization techniques, mechanical properties similar to the native vessel, low cost, and availability. The following section will discuss the various polymers considered and additional details supporting the final material selection.

\subsection{Discussion}

One main criterion for material selection was to use a polymer that had previously been electrospun. Natural materials considered were collagen and elastin. Both materials can be electrospun together or independently and are advantageous since they are completely biologic. However, these materials lack the necessary mechanical properties needed for the BVM. Natural and synthetic matrices such as PCL/collagen, PLGA/collagen, and PLGA/collagen/elastin attain comparable mechanical properties to the native vessel with favorable EC and SMC responses. PCL / collagen showed promising results in vascular tissue development in a pulsatile perfusion bioreactor followed by implantation into a rabbit model. The scaffold was stable one month after implantation [120]. However, electrospun PCL without collagen from another study showed a great degree of compliance mismatch [121]. PLGA was effectively co-spun with collagen and elastin to achieve better compliance and burst strength [56]. Electrospun PLGA with collagen alone, cultured in a pulsatile, perfusion bioreactor enhanced vascular EC attachment and retention of cell phenotype [128]. Unfortunately, the high cost of collagen and the more complicated networks could be restrictive to the BVM model in terms of cost and scalability. 
PLGA without the addition of natural polymers showed excellent results for vascular tissue development using HUVECs [127]. Another PLGA study achieved $>90 \%$ porosity with nano-sized fiber diameters which consequently encouraged good fibroblast attachment [109]. PCL and PLGA can be gas sterilized. Other polymers studied for the potential in vascular tissue engineering were PDO and PGA. Both polymers have fast degradation rates. Nonbiodegradable polymers are preferred for the BVM model in order to maintain structural integrity during endothelialization and intravascular device evaluation. Such fast degrading polymers would be inadequate for the design. Non-biodegradable polyurethanes can achieve compliance similar to the native vessel [130], but polyurethanes are generally expensive, hard to find, and variable in composition. However, if progress is made towards the addition of SMCs, in addition to ECs, a faster degrading polymer might be acceptable for the BVM model. Polymers with relatively slow degradation rates are possible as long as degradation does not affect the integrity of the construct before the end of device evaluation ( 3-4 weeks).

In conclusion, PLGA was determined to be the most acceptable and appropriate polymer for the investigation into electrospinning scaffolds for the BVM. In summary, PLGA's ability to be electrospun into fibrous, porous constructs, and its ability to elicit appropriate cellular responses under physiological pulsatile flow environments make the polymer suitable for vascular tissue engineering applications. PLGA is a relatively inexpensive polymer that can be EtO gas sterilized in house. PLGA's compatibility for co-spinning with biologics, although not an objective of this thesis, could be beneficial for opening up other areas of research and design. 


\section{Chapter 3 - Electrospinning PLGA}

\subsection{Introduction}

PLGA was determined to be the most appropriate polymer for the investigation of electrospun scaffolds for the BVM. Additionally, PLGA is one of the most well studied synthetic polymers for tissue engineering of various tissue constructs [131-133]. The FDA has approved the use of PLGA in several biomedical applications due to good biocompatibility and controlled biodegradability $[131,132]$. PLGA is a co-polymer of lactic and glycolic acid, and degradability can be tailored for targeted applications via the ratio of acid units. Degradation of PLGA occurs by hydrolysis of the ester linkages in the presence of water producing by-products of lactic and glycolic acid, both of which are by-products of metabolic pathways under normal physiological conditions. Degradation however is a concern for the BVM model. The model necessitates scaffold structural stability to support an endothelium throughout the course of intravascular device evaluation. In order to incorporate a biodegradable polymer into the BVM design, the degradation rate of the polymer must be sufficiently slow (3.4 weeks) to allow for complete device testing.

In vitro and in vivo breakdown rates of electrospun PLGA scaffolds with mole fractions of 85:15, 75:25 and 50:50 were compared by Blackwood et al [134]. PLGA 85:15 had no significant change in vitro after 108 days. PLGA 75:25 did not begin rapid degradation for 80 days, whereas PLGA 50:50 underwent rapid degradation within 8 days. In vivo subcutaneous implantation of electrospun PLGA scaffolds also confirmed the potential use of 85:15 and 75:25 for tissue engineering. The study noted good fibroblast growth, EC growth, and ECM production for 85:15 and 75:25 in vivo, with significant degradation after 3-4 months of 
implantation [134]. From the results of Blackwood et al., it can be assumed that degradation of both PLGA 85:15 and PLGA 75:25 are slow enough to be used in the BVM model. Also, incorporation of SMCs to act as the media of the vessel may necessitate such biodegrading polymers, in which case the scaffold no longer provides the long term structural support as a layer of smooth muscle is created. In conclusion, PLGA 75:25 was selected for the remainder of this investigation. Although both PLGA 85:15 and 75:25 are viable options, the 75:25 ratio has been used more extensively in literature.

PLGA meets other BVM criteria listed in the objective of this investigation as outlined in Chapter 2. The purpose of this chapter is to develop a protocol for electrospinning PLGA. Solvents, solution concentration, and processing parameters (flow rate and applied voltage) will be investigated for optimization of construct morphology and mechanical properties of the PLGA scaffolds. Once a protocol is finalized, PLGA scaffolds will be developed at the final protocol for evaluation in the BVM bioreactor to test the ability to form a human endothelial cell lining.

\subsection{Methods}

\subsubsection{Solvent Selection}

Chloroform $\left(\mathrm{CHCl}_{3} ; 99.9 \%\right.$, extra dry, water < 50 ppm; mol wt. 119.38; Cat. No. AC32682-0010_Acros Organics No.: 326820010), tetrahydrofuran (THF; anhydrous > 99.9\%, inhibitor free; Cat. No. 401757) and dimethylformamide (DMF; anhydrous, 99.8\%; Cat. No. 227056) were purchased from Fisher Scientific and Sigma-Aldrich Chemical Company. Poly(D,L-lactide-co-glycolide) [75:25; PLGA; mol wt. 66,000 - 107,000; Cat. No. P1941] was purchased from Sigma Life Sciences. Chloroform, THF, and DMF were considered based on 
primary literature to determine an appropriate solvent for electrospinning PLGA. Comparisons were made based upon cost, volatility, and primary literature review on electrospinning. In addition, $10 \mathrm{wt} \%$ and $15 \mathrm{wt} \%$ solutions of PLGA in THF and PLGA in $\mathrm{CHCl}_{3}$ were made according to the protocol in Appendix B. All four solutions were electrospun according to the protocol in Appendix C. The four spins were used as trial spins to monitor effectiveness of solvents to bring PLGA resin into solution and to visually evaluate the ability to electrospin each solution. All spins were conducted with an 18 GA needle, and a gap distance of $25.4 \mathrm{~cm}$. Mandrel rotation control was set to 6 and translation was set to 3. Flow rate and applied voltages were varied during the spin process as each spin was solely observational and experimental.

\subsubsection{Solution Concentration}

Poly(D,L-lactide-co-glycolide) [75:25; PLGA; mol wt. 66,000 - 107,000; Cat. No. P1941] was purchased from Sigma Life Sciences. Using the solvent determined appropriate for electrospinning PLGA in the previous section, an optimum weight percent solution concentration was investigated. Solution concentration was varied between $10 \mathrm{wt} \%$ and $18 \mathrm{wt} \%$ PLGA in $\mathrm{CHCl}_{3}$. Each solution was prepared according to the protocol provided in Appendix B. Calculations to determine grams of polymer necessary for a given wt $\%$ solution were conducted based on $4 \mathrm{ml}$ solvent (See Appendix B). Each solution was electrospun according to the protocol provided in Appendix C. For each spin, $3 \mathrm{ml}$ of solution was electrospun out of the 4 $\mathrm{ml}$ solution made. Mandrel rotation control was set to 6 and translation was set to 3. Table III provides the specific solution and processing parameters set for each different wt $\%$ solution tested. Flow rates and voltages were adjusted specifically based upon the response of droplet and jet formation at the start of the spin process. This was necessary since at this time no protocol for electrospinning PLGA had been determined and each spin was experimental. With 
the completion of each spin, the scaffold was left on the mandrel in a desiccator for at least 24 hours in order to eliminate any excess solvent prior to scaffold removal and analysis. All applied voltages listed are approximations since no exact measurement system is in place for the electrospinning setup.

Table III - Solution and Electrospinning Parameters for PLGA in Chloroform

\begin{tabular}{c|ccc|cccc}
\hline & \multicolumn{3}{|c|}{ Solution Parameters } & \multicolumn{4}{|c}{ Electrospinning Parameters } \\
\hline $\begin{array}{c}\text { Spin } \\
\text { Title }\end{array}$ & $w t \%$ & $\begin{array}{c}P L G A \\
(\mathrm{~g})\end{array}$ & $\begin{array}{c}\mathrm{CHCl}_{3} \\
(\mathrm{ml})\end{array}$ & $\begin{array}{c}\text { Gap Distance } \\
(\mathrm{cm})\end{array}$ & $\begin{array}{c}\text { Needle } \\
(\mathrm{GA})\end{array}$ & $\begin{array}{c}\text { Flow Rate } \\
(\mathrm{ml} / \mathrm{hr})\end{array}$ & $\begin{array}{c}\text { Applied } \\
\text { Voltage }(\mathrm{V})\end{array}$ \\
\hline SPIN 1 & 10 & 0.653 & 4 & 25.4 & 18 Blunt & 6.1 & no data record \\
SPIN 2 & 12 & 0.806 & 4 & 25.4 & 18 Blunt & 2.5 & 10,500 \\
SPIN 3 & 13 & 0.888 & 4 & 25.4 & 18 Blunt & 3 & 10,500 \\
SPIN 4 & 15 & 1.031 & 4 & 25.4 & 18 Blunt & 5.5 & no data record \\
SPIN 5 & 18 & 1.201 & 4 & 25.4 & 18 Blunt & $0.1-6.0$ & $10-20,000$
\end{tabular}

\subsubsection{Morphological Analysis}

Electrospun PLGA scaffolds, SPIN 1-5, were evaluated using scanning electron microscopy (SEM) for qualitative analysis of construct morphology. Keeping the scaffold on the mandrel for support, each scaffold was cut into 3 sections (approximately $4 \mathrm{~cm}$ long each) with a scalpel, and sections were labeled A-B, B-C, and C-D (Figure 9). One sample from each section, approximately $3 \mathrm{~mm}$ wide, was cut and taken off the mandrel. First a sample from A-B was cut and removed, and then what was left of A-B was removed and placed on a glass stir rod. This was repeated for all sections. The glass stir rod with leftover sections was placed back in the desiccator and labeled appropriately with spin title, date and wt $\%$ concentration. The $3 \mathrm{~mm}$ samples were then cut in half so both lumen and ablumen could be analyzed with the SEM. Samples were fixed to a SEM stage and sputter coated in gold for approximately 60 seconds 
(Figure 9). Qualitative analysis of electrospun PLGA construct morphologies was conducted using the SEM and several images were taken at various magnifications.

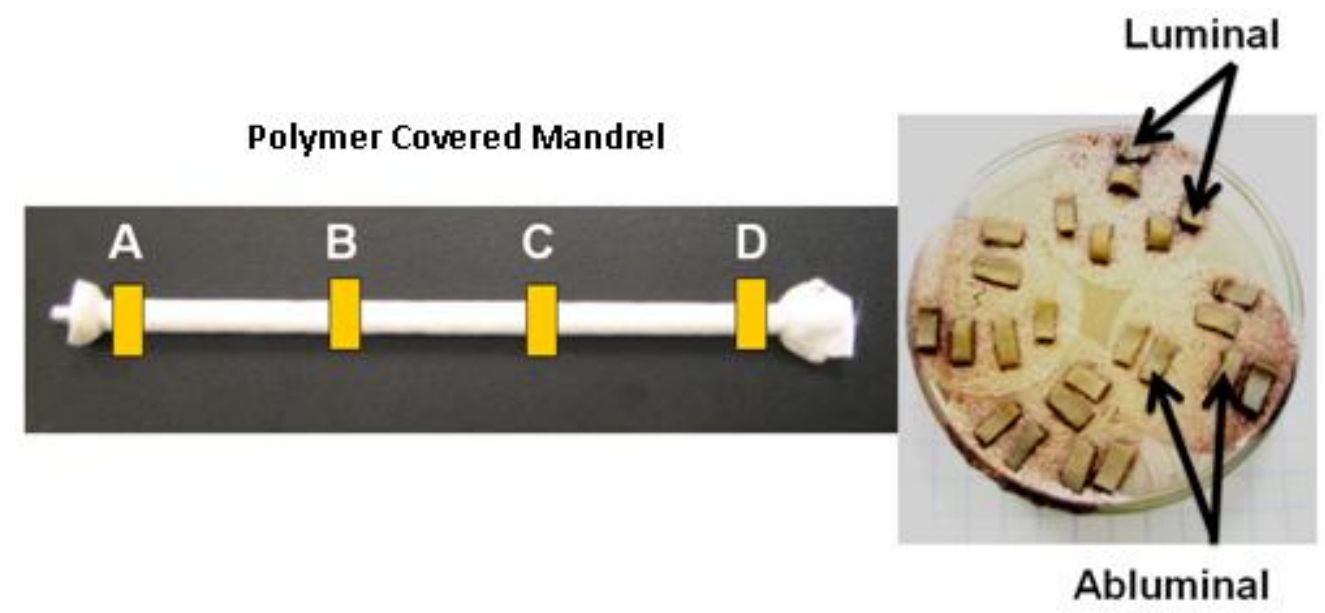

Figure 9 - The above images are example images and do not display SPIN 1 - 5. The left image shows polymer covering the mandrel after electrospinning. The labeling method is shown. The large SEM stage shows both lumen and ablumen samples sputter coated in gold (right). All electrospun samples were prepared in this manner for SEM.

\subsubsection{Processing Parameter Optimization}

After an optimum wt\% concentration was determined, fine tuning of applied voltage and flow rate was conducted. Poly(D,L-lactide-co-glycolide) [75:25; PLGA; mol wt. 66,000 107,000; Cat. No. P1941] from Sigma Life Sciences and chloroform $\left(\mathrm{CHCl}_{3} ; 99.9 \%\right.$, extra dry, water <50 ppm; mol wt. 119.38; Cat. No. AC32682-0010_Acros Organics No.: 326820010) from Fisher Scientific were used for this experiment. Four $15 \mathrm{wt} \%$ solutions of PLGA in $\mathrm{CHCl}_{3}$ were prepared according to Appendix B. Calculations to determine grams of polymer necessary for a given wt $\%$ solution were conducted based on $4 \mathrm{ml} \mathrm{CHCl}_{3}$. Each solution was electrospun according to Appendix C. Only $3 \mathrm{ml}$ of the $4 \mathrm{ml}$ solution was electrospun for each spin. Mandrel rotation was set to 6 and translation set to 3 for all spins. Table IV provides the specific 
processing parameter for each of the 4 spins. Each applied voltage is an estimate since no exact measuring system is in place. A temperature and humidity monitor was kept in the containment chamber and both were recorded at the beginning and end of each spin to monitor affects if any. Only temperature and humidity changes of \pm 5 units were considered serious to note, otherwise values displayed are an average from start to finish of each spin.

Table IV - Electrospinning Process Parameters for $15 \mathrm{wt} \%$ PLGA in $\mathrm{CHCl}_{3}$

\begin{tabular}{c|c|cccccc}
\hline & $\begin{array}{c}\text { Solution } \\
\text { Parameters }\end{array}$ & \multicolumn{7}{|c}{ Electrospinning Parameters } \\
\hline $\begin{array}{c}\text { Spin } \\
\text { Title }\end{array}$ & PLGA $(\mathrm{g})$ & $\begin{array}{c}\text { Gap } \\
\text { Distance } \\
(\mathrm{cm})\end{array}$ & $\begin{array}{c}\text { Needle } \\
(\mathrm{GA})\end{array}$ & $\begin{array}{c}\text { Flow Rate } \\
(\mathrm{ml} / \mathrm{hr})\end{array}$ & $\begin{array}{c}\text { Applied } \\
\text { Voltage } \\
(\mathrm{V})\end{array}$ & $\begin{array}{c}\text { Temperature } \\
\left({ }^{\circ} \mathrm{F}\right)\end{array}$ & $\begin{array}{c}\text { Humidity } \\
(\%)\end{array}$ \\
\hline SPIN 6 & 1.036 & 25.4 & 18 Blunt & 5.5 & 12,000 & 66.1 & 47 \\
SPIN 7 & 1.031 & 25.4 & 18 Blunt & 3 & 12,000 & 69.0 & 40 \\
SPIN 8 & 1.047 & 25.4 & 18 Blunt & 3 & 15,600 & 68.4 & 38 \\
SPIN 9 & 1.038 & 25.4 & 18 Blunt & 5.5 & 15,600 & 68.2 & 37
\end{tabular}

\subsubsection{Fiber Diameter Measurements}

SEM was used to both qualitatively evaluate general construct morphology and quantitatively measure fiber diameter of the luminal surface of the scaffold. SPIN 6-9 were prepared in the same manner as mentioned previously in section 3.2.2.1 for SEM analysis. Following that protocol, a total of 3 luminal and 3 abluminal samples were sectioned per spin. Both lumen and ablumen were observed for a general consistency using the SEM, however, the luminal surface was of greatest concern for fiber diameter measurements. One corner of each lumen sample was selected to start image collection. All images were acquired at a magnification of 2000X. Images were acquired every 20 screens from the selected corner. Five 
images were taken per lumen sample per spin, for a total of 15 images per spin. A program called ImageJ was used to measure fiber diameters from the SEM images (Figure 10). The program converts number of pixels into a length scale. Fiber diameters were measured according to the protocol in Appendix E. Approximately 5 fiber diameter measurements were taken per image for a total of 75 measurements per spin. Average fiber diameter and standard deviation were calculated in Excel.

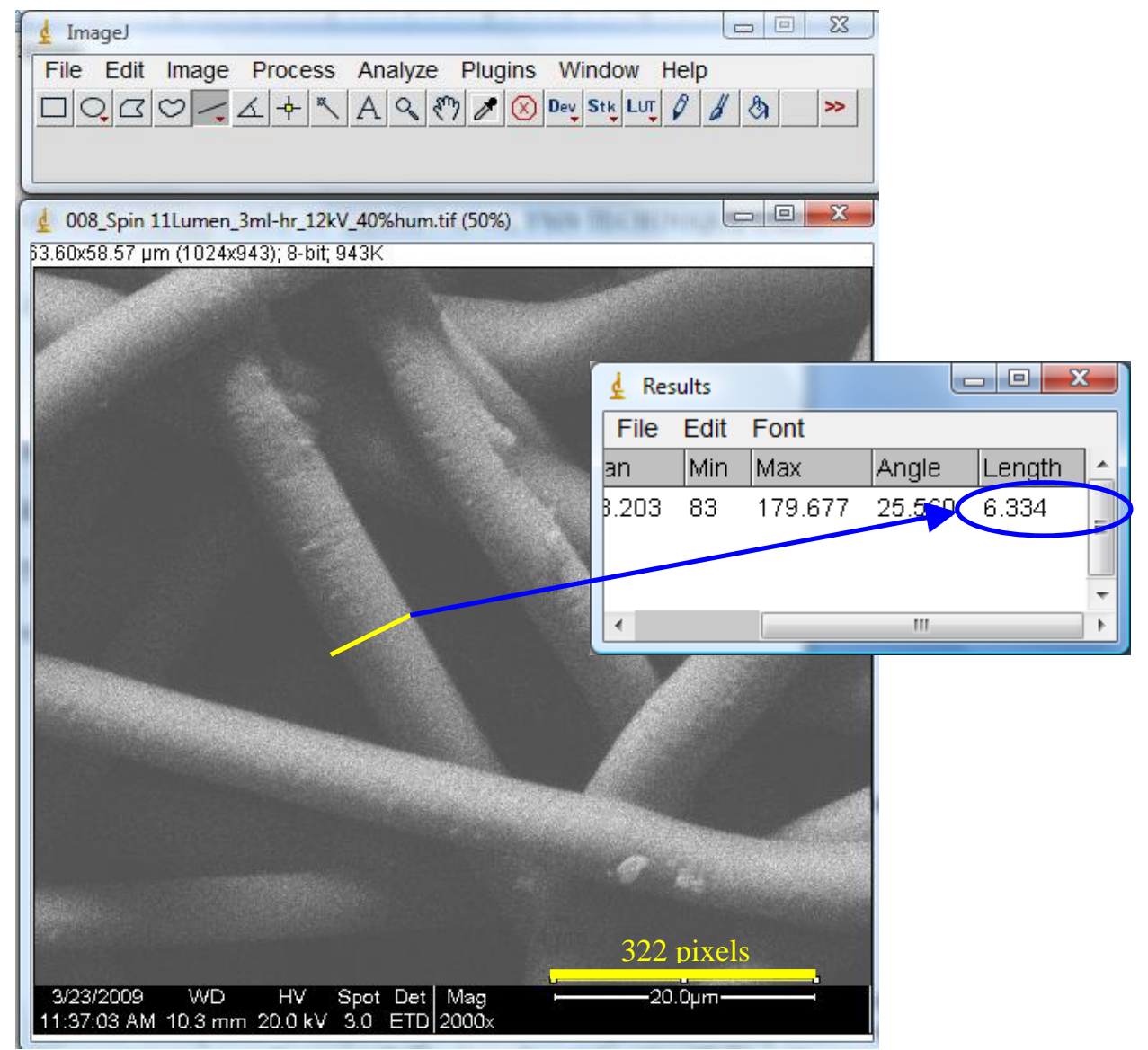

Figure 10 - ImageJ can be used to convert pixels into a length scale. The above SEM image shows a fiber diameter of 6.334 microns reported by ImageJ output. 


\subsubsection{Mechanical Testing}

Mechanical properties of $15 \mathrm{wt} \%$ PLGA electrospun scaffolds were measured using a uniaxial tensile tester (Instron In-Spec 2200 Portable Tester with PDA Data Management Software) equipped with a $250 \mathrm{~N}(50 \mathrm{lb})$ load cell. Section A-B, from SPIN 6-9, as described in section 3.2.2.1, were used for this test. Each A-B section was cut for both longitudinal and circumferential tensile testing using a scalpel or scissors. Two samples were cut to be tested longitudinally and one sample was cut for circumferential testing as shown in the schematic illustration below (Figure 11). Circumferential samples were cut once along the scaffold longitudinal axis and rolled out flat for testing.

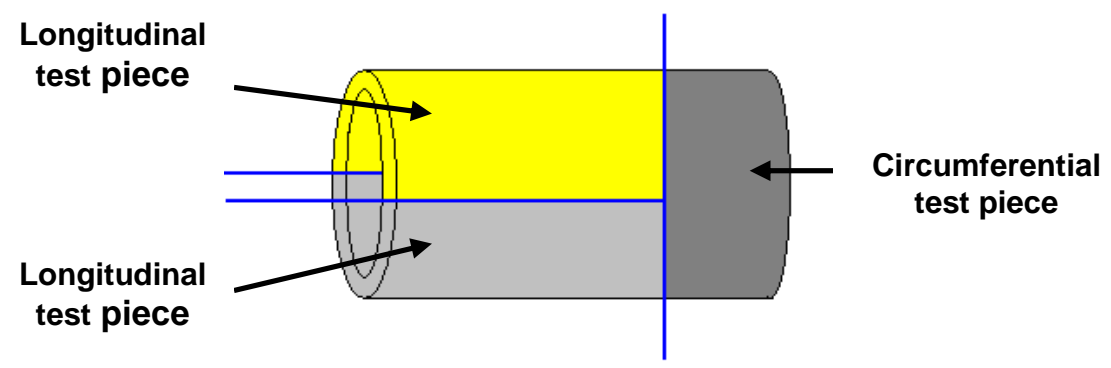

Figure 11 - Illustration of section A-B from each SPIN prepared for tensile testing. Blue lines represent cutting lines. 2 samples were created for longitudinal testing (yellow and light gray) and one sample for circumferential testing (dark grey).

Each sample was loaded into the Instron test grips in the proper test direction. Sample dimensions (gage length, width, and thickness) were measured using digital calipers. The data acquisition system reported time, extension, and load. Extension and load were converted to stress and strain in Excel. The ultimate tensile strength and Young's Modulus were obtained 
from the stress-strain curves. Young's modulus was calculated from the slope of the initial linear region of the stress strain curve.

\subsection{Results}

\subsubsection{Solvent Selection}

Chloroform was determined an acceptable solvent for electrospinning PLGA for the remainder of this investigation. Reasons for selecting chloroform are cost, adequate volatility, and the ability to bring PLGA resin into solution. DMF was considered because it is often used in combination with THF or chloroform $[78,133,135]$. Boiling points for THF and chloroform are $60.5-61.5^{\circ} \mathrm{C}$ and $65-67^{\circ} \mathrm{C}$ respectively, making both equally viable options with THF being slightly more volatile. Chloroform is approximately $\$ 12.00-20.00$ cheaper than both THF and DMF and has been used extensively in literature for electrospinning various polymers $[78,87$, 136, 137]. A price comparison table is shown below.

Table V - Price Comparisons for Different Solvents

\begin{tabular}{|c|c|c|}
\hline & $100 \mathrm{ml}$ & 1Liter \\
\hline Chloroform & $\$ 25.50$ & $\$ 52.10$ \\
\hline THF & $\$ 38.30$ & $\$ 79.80$ \\
\hline DMF & $\$ 37.60$ & $\$ 73.30$ \\
\hline
\end{tabular}

*Prices obtained from Sigma-Aldrich and Fisher Science

Both THF and $\mathrm{CHCl}_{3}$ effectively brought PLGA resin into solution according to the protocol in Appendix B. Electrospinning of $10 \mathrm{wt} \%$ PLGA in THF was unsuccessful and no scaffold construct was formed. Electrospun $15 \mathrm{wt} \%$ PLGA in THF resulted in electrospraying. 
Both 10 and $15 \mathrm{wt} \%$ solutions of PLGA in $\mathrm{CHCl}_{3}$ were successfully electrospun with polymer collection evident on the mandrel.

\subsubsection{Solution Concentration}

This section is provided to give a record of observations seen during and at the end of SPIN 1-5. Given this was one of the first experimental investigations using the electrospinning device, several observations and errors were recorded and explanations of observations are included in the discussion section of this chapter.

SPIN 1: At the start of SPIN 1, $10 \mathrm{wt} \%$ PLGA, the mandrel and rotator cuff was not working when the rotor was turned on. By tapping the rotator cuff, mandrel rotation was initiated and the rotator cuff unstuck. During the spin, a large droplet of polymer solution formed at the tip of the capillary. Droplet size would reach an unstable size and fall to the floor. Also, multiple fiber jets were coming from the droplet. Usually excess fiber jets scattered about the collector and dried separate from the mandrel. The voltage at which the spin was conducted was not recorded, however the process was most likely conducted between 10,000 and 20,000 V. Also, the lab did not have access to a desiccator until SPIN 2 and the scaffold was kept in a plastic zip lock bag until further analysis.

SPIN 2: The flow rate was reduced for SPIN $2,12 \mathrm{wt} \%$ PLGA, to $2.5 \mathrm{ml} / \mathrm{hr}$ in order to possibly reduce the amount of polymer lost to a high flow rate as observed during SPIN 1. With the start of the electrospinning process, the mandrel was quickly coated with the polymer and a stable jet formed from the tip of the needle. However, an additional jet formed that solidified onto the ceiling of the containment chamber. 
SPIN 3: Since SPIN 3, 13 wt\% PLGA, was slightly higher in concentration than SPIN 2, a similar flow rate was maintained $(3 \mathrm{ml} / \mathrm{hr})$. Again, polymer collection on the mandrel was immediately evident as in SPIN 2. Not long into the spin, the polymer droplet began to grow larger and hang from the tip of the capillary. The droplet was elongated in shape, however, a stable jet continued from the tip of the elongated droplet. When the droplet grew too large, it would fall to floor of the containment chamber and a new jet would initiate.

SPIN 4: For SPIN 4, 15wt\% PLGA, a flow rate of $5.5 \mathrm{ml} / \mathrm{hr}$ was attempted in order to spin a full scaffold in less amount of time. No voltage was recorded for this spin. However, it was recorded that increasing the voltage led to multiple jet formations. The voltage was then decreased until a stable jet was observed.

SPIN 5: The SPIN at $18 \mathrm{wt} \%$ PLGA was conducted as a troubleshooting experiment, as well as to see the affects of solution concentration on construct morphology. Flow rate was varied between $0.1 \mathrm{ml} / \mathrm{hr}$ and $6.0 \mathrm{ml} / \mathrm{hr}$ and voltage varied between 10,000-20,000 V. The purpose of the variation was to see if any observable increase in droplet stability could be reached by changing the flow rate and voltage parameters. Flow rate was started at $6.0 \mathrm{ml} / \mathrm{hr}$ and the voltage was adjusted. When the voltage was too high, multiple jets formed from the droplet and or the droplet became concave inward and circular with multiple jets forming around the outer diameter. If the voltage was too low, a jet would cease to form and polymer solution would drip from the tip of the capillary. When voltage seemed to stabilize the jet formation, a large droplet began to form. The flow rate was reduced slowly until $0.1 \mathrm{ml} / \mathrm{hr}$ to see if any significant difference in droplet size was evident. The droplet size did decrease with reduced flow rate, but remained throughout the entire spin. 


\subsubsection{Morphological Analysis}

Morphological examination using SEM images of SPIN 1, 2, 3, 4 and 5 are shown below in Figures 13-20. At 10, 12 and 13 wt\% PLGA, fiber morphologies contained rounded and elongated bead defects interconnected with nano-sized fiber diameters (Figure 12,Figure 13Figure 14,Figure 15). SPIN 4 and 5, at 15 and $18 \mathrm{wt} \%$ PLGA respectively, had continuous un-beaded fiber networks with fiber diameters of just a few microns (Figure 16Figure 17, Figure 18, Figure 19). Figure 20 shows the continuity of the electrospun fibers however, there appears to be no preferential direction of the fibers. Note images were taken at different magnifications. Based on SEM analysis, the optimum solution concentration for the continuation of this study was $15 \mathrm{wt} \%$ PLGA in $\mathrm{CHCl}_{3}$.

It should be noted that SPIN 1 was difficult to detach from the mandrel and parts of the scaffold were damaged in the process. Unlike SPIN 1, SPIN 2, 3, 4 and 5 were maintained in a desiccator until removal from the mandrel. The use of desiccant allowed for easier removal of polymer from the mandrel by drying excess solvent. There was a noticeable increase in stiffness for all scaffolds maintained in the desiccator. 


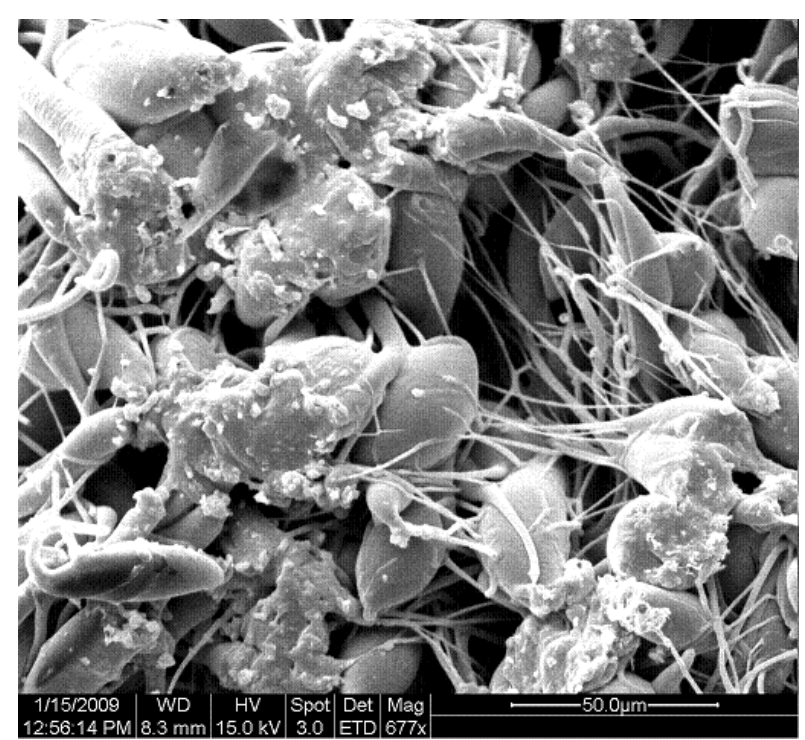

Figure 12 - SEM image of SPIN 1, $10 \mathrm{wt} \%$ PLGA, electrospun construct showing globular bead defects amongst small fibers (677X).

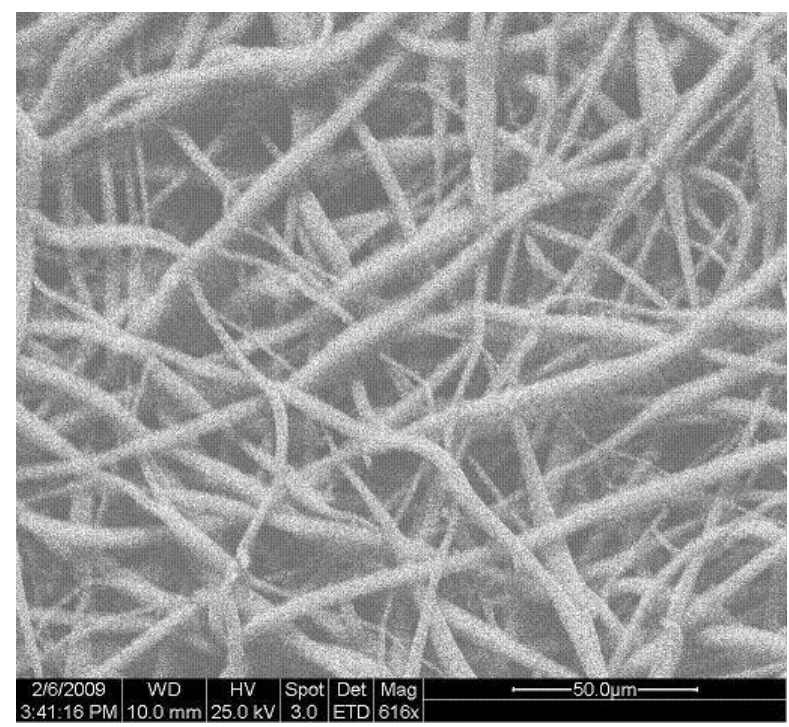

Figure 14 - SEM image of SPIN 2, 12 wt\% PLGA, electrospun construct with elongated beads on fibers $(615 \mathrm{X})$.

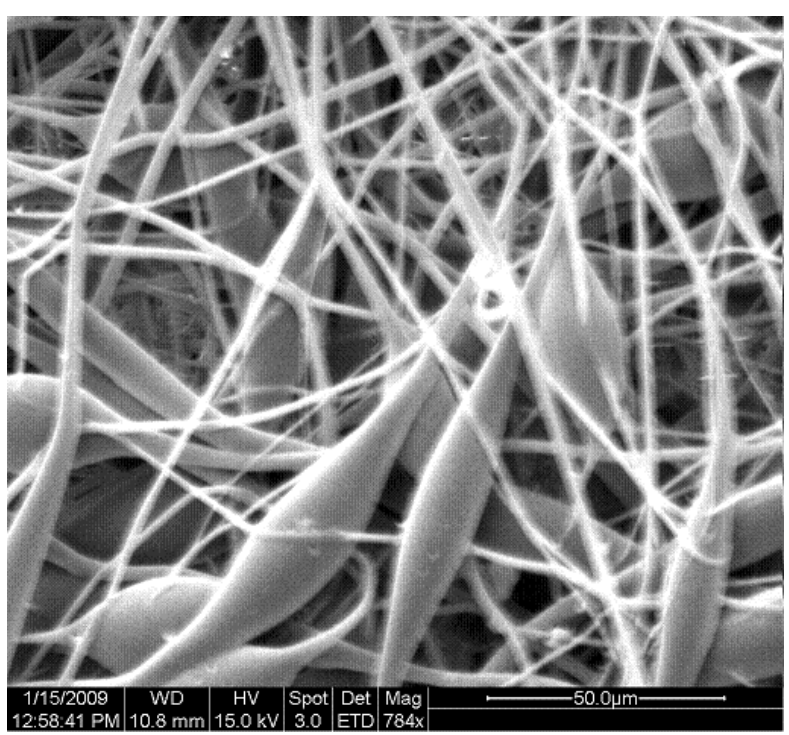

Figure 13- SEM image of SPIN 1, 10 wt \% PGLA, electrospun construct with elongated bead defects among small fibers (784X).

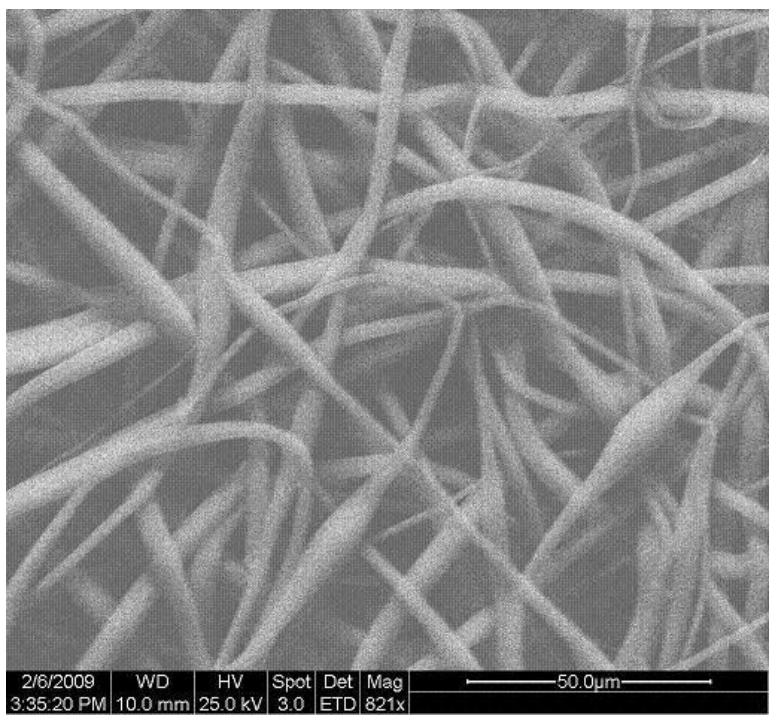

Figure 15 -SEM image of SPIN 3, 13 wt\% PLGA, electrospun construct with few elongated beads and varying fiber sizes (821X). 


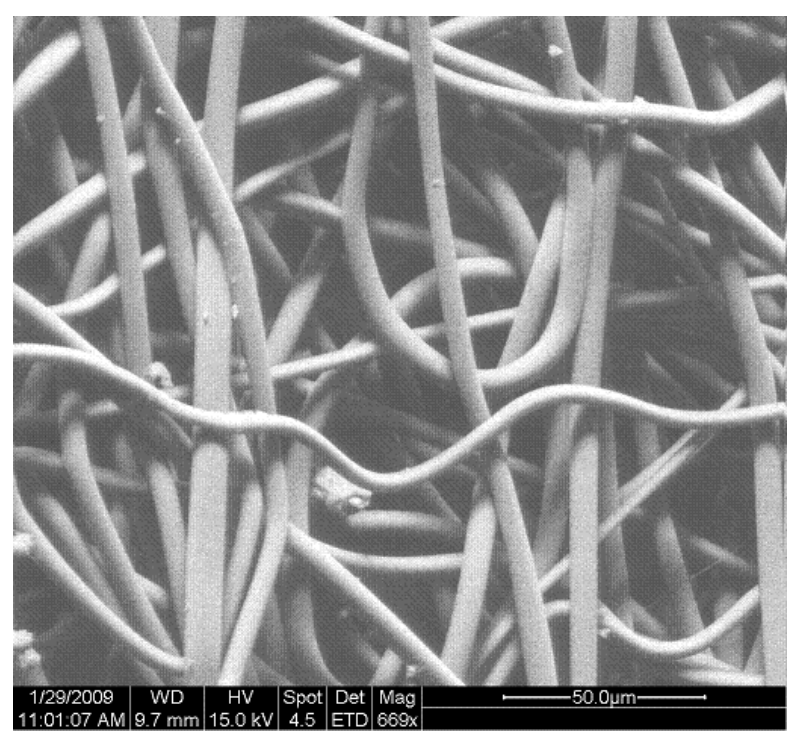

Figure 16 - SEM image of SPIN 4, 15 wt\% PLGA, electrospun construct with continuous fiber network (669X).

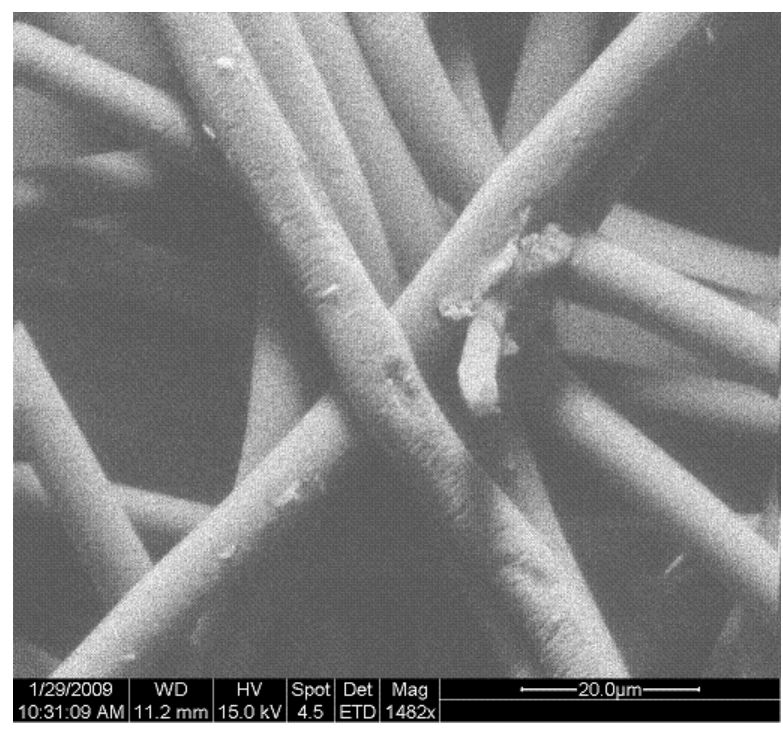

Figure 18 - SEM image of SPIN 5, 18wt $\%$

PLGA, electrospun construct with continuous fibers (1482X).

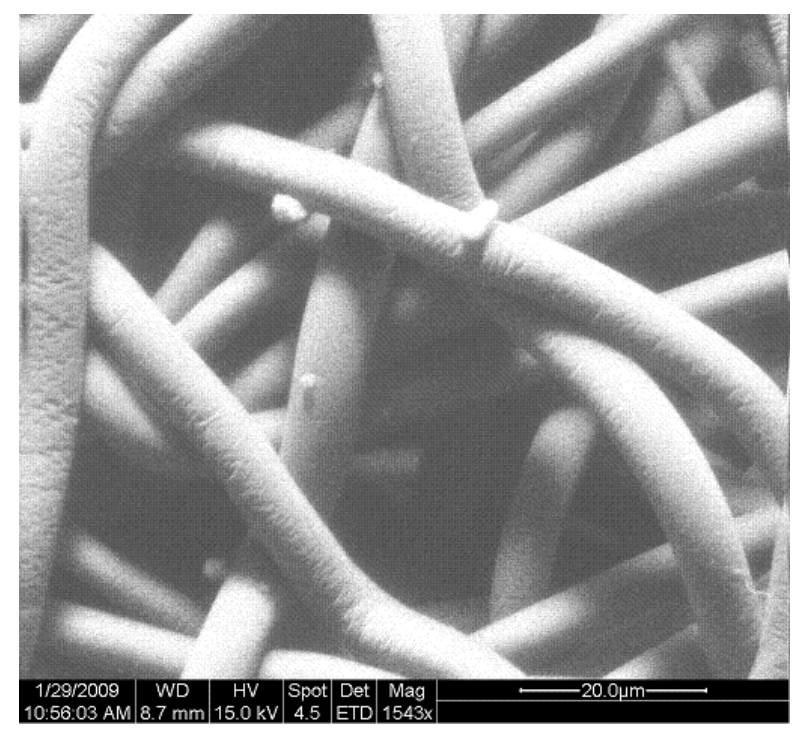

Figure 17 - SEM image of SPIN 4, 15 wt\% PLGA, electrospun construct with continuous fibers (1543X).

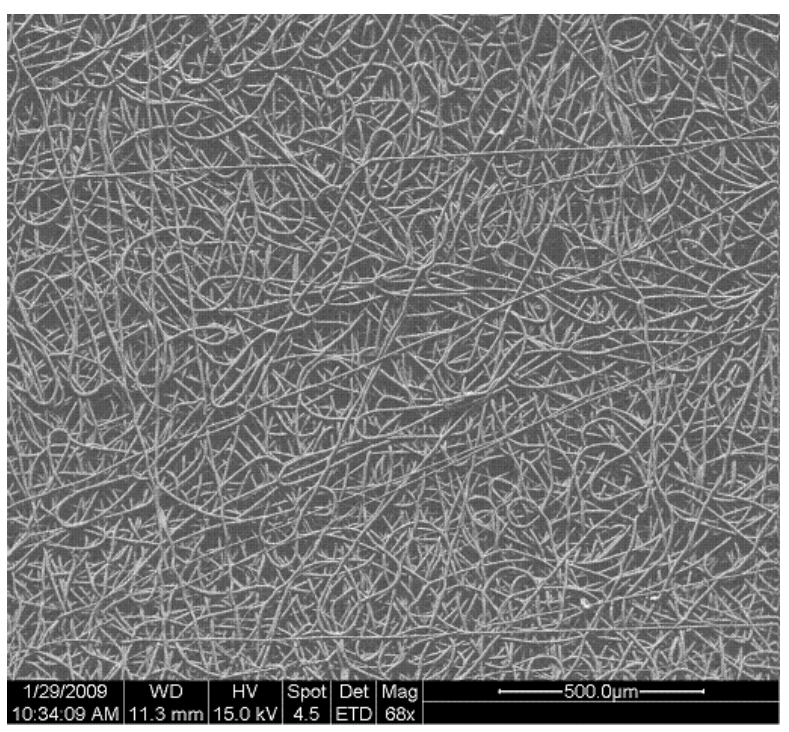

Figure 19 - SEM image of SPIN 5, 18 wt\% PLGA, electrospun constructs with continuous fibers $(\mathbf{6 8 X})$ 


\subsubsection{Processing Parameter Optimization}

The purpose of this section is to report any observations or error that occurred during the electrospinning of SPIN 6, 7, 8 and 9. Again, this is the second run of trial spins investigated and it was therefore important to record any interesting observations, as well as reasons for parameter adjustments. Results of each spin are presented below and will be further discussed in the discussion section of this chapter.

SPIN 6: At the beginning of SPIN 6 the temperature and humidity were recorded to monitor effects, if any. This was not considered for SPIN 1-5 and no environment data exists for those spins. Polymer coating of the mandrel began immediately at the start of the electrospinning process for SPIN 6. An extruded droplet formed at the tip of the capillary; however a stable jet was formed at the tip of the droplet. After $1 \mathrm{ml}$ of solution was spun, the process was stopped and restarted with a flow rate of $3.5 \mathrm{ml} / \mathrm{hr}$ and voltage was maintained at $12,000 \mathrm{~V}$. At the slower flow rate there was no difference in droplet size formation. Due to that fact, the flow rate was returned to $5.5 \mathrm{ml} / \mathrm{hr}$ for the remainder of the spin.

SPIN 7: Parameters for SPIN 7 were set to $3 \mathrm{ml} / \mathrm{hr}$ and 12,000 V. Parameters remained constant throughout the entire spin process. Even with a lower flow rate, similar polymer build up, as in SPIN 6, was observed.

SPIN 8: The purpose of SPIN 8 was to test a lower flow rate $(3 \mathrm{ml} / \mathrm{hr})$ with an increase in voltage. Voltage began at $12,600 \mathrm{~V}$ but was soon amped up to $18,000 \mathrm{~V}$. In both cases the jet was unstable; however a decrease in voltage to $15,600 \mathrm{~V}$ produced a stable jet formation. 
SPIN 9: The purpose of SPIN 9 was to test a higher flow rate $(5.5 \mathrm{ml} / \mathrm{hr})$ with the voltage found to stabilize the jet during SPIN $8(15,600 \mathrm{~V})$. All parameters were kept constant the entire spin.

For each spin, humidity and temperature were recorded. Humidity was highest for SPIN 6 at $47 \%$. When observing the collected polymer on the mandrel at the end of SPIN 6, the collected polymer appeared wet or wavier than what has previously and afterwards been observed. This suggests minor changes in humidity may play a role in complete solvent evaporation during the electrospinning process.

\subsubsection{Fiber Diameter Measurements}

SPIN 6, 7, and 8 were difficult to remove from the mandrel during SEM sample preparation. This could be due to excessive use of the original mandrel. Also, using a scalpel to cut samples off the mandrel leaves grooves in the mandrel surface. These grooves could act as areas for polymer to become wedged making removal more difficult. SPIN 9 was electrospun onto a new mandrel made in-house (See Appendix D). The scaffold came off with no difficulty and was possibly the easiest scaffold to remove thus far. New mandrel designs were wet polished in methanol. Smooth surfaces achieved from this process could have aided in scaffold removal. New mandrels will be used for all future spins.

SEM images of SPIN 6-9 are shown below in Figures 21-28. Images showing both lumen and ablumen morphologies are displayed. Results of fiber diameter measurements are listed in Table VI. SPIN 6 achieved the smallest average fiber diameter of 5.49 microns. SPIN 8 achieved the largest average fiber diameter of 7.35 microns. Raw data collected for all fiber diameter measurements is included in Appendix F. It is important to note, the method of fiber 
diameter measurements is not entirely accurate due to apparent verses true fiber diameter. Small fibers may change more in their spatial orientation. Meaning, a fiber that appears straight in a SEM image may actually be turning into or out of the page. For this reason average measured fiber diameter is potentially greater than the actual average diameter of each electrospun construct being observed. This possibility was not accounted for in the statistical analysis and data collection, and is assumed an insignificant effect.

Table VI - Fiber Diameter Results for 15 wt\% PLGA Electrospun Scaffolds

\begin{tabular}{|c|c|c|c|c|}
\hline \multirow[b]{2}{*}{ Spin Title } & \multirow{2}{*}{$\begin{array}{c}\text { Fiber Diameter } \\
(\text { um })\end{array}$} & \multirow{2}{*}{$\begin{array}{l}\text { Standard } \\
\text { Deviation }\end{array}$} & \multicolumn{2}{|c|}{ Electrospinning Parameters } \\
\hline & & & $\begin{array}{c}\text { Flow Rate } \\
(\mathrm{ml} / \mathrm{hr})\end{array}$ & $\begin{array}{c}\text { Applied Voltage } \\
(V)\end{array}$ \\
\hline SPIN 6 & 5.49 & 0.93 & 5.5 & 12,000 \\
\hline SPIN 7 & 5.85 & 0.64 & 3.0 & 12,000 \\
\hline SPIN 8 & 7.35 & 1.00 & 3.0 & 15,600 \\
\hline SPIN 9 & 6.11 & 0.75 & 5.5 & 15,600 \\
\hline
\end{tabular}




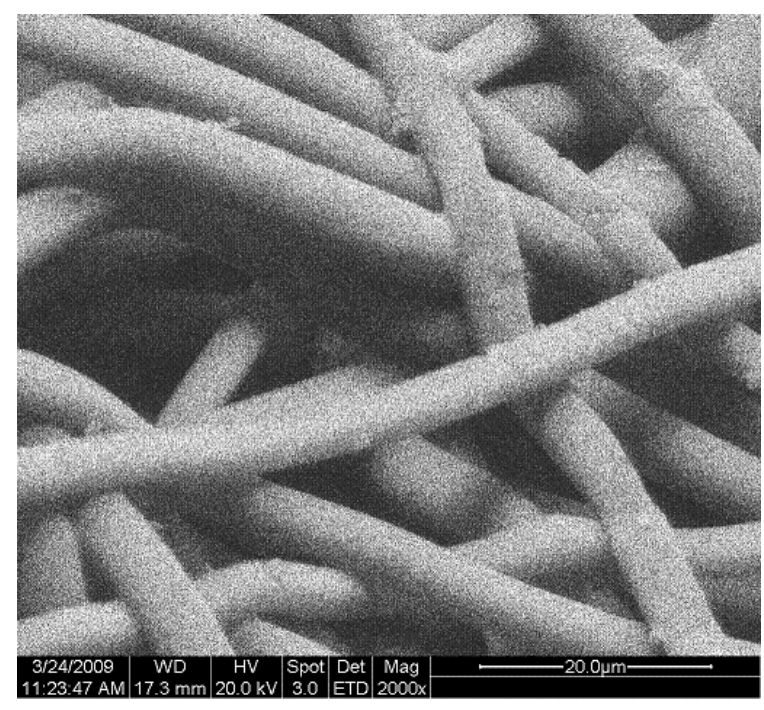

Figure 20 - SEM image of SPIN 6, lumen of PLGA electrospun construct (2000X).

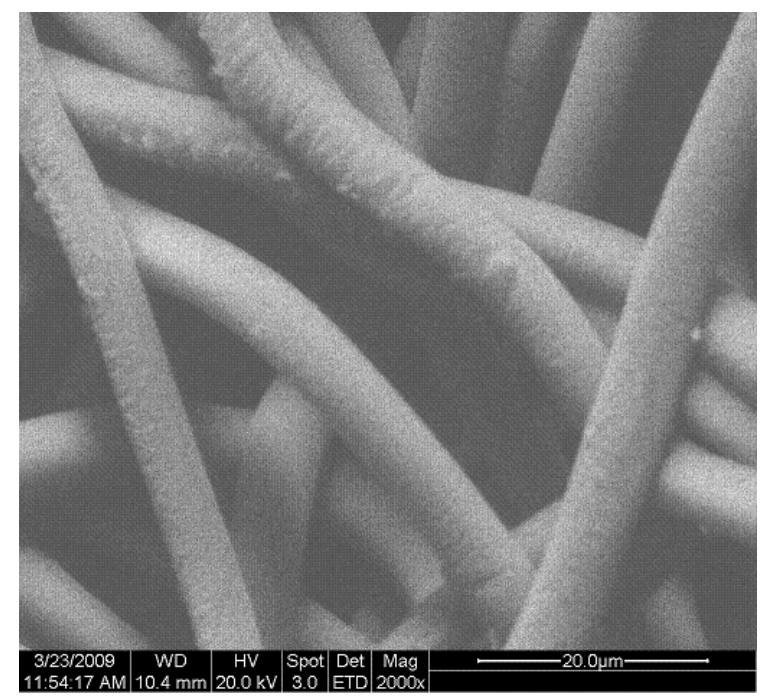

Figure 22 - SEM image of SPIN 7, lumen of PLGA electrospun construct (2000X).

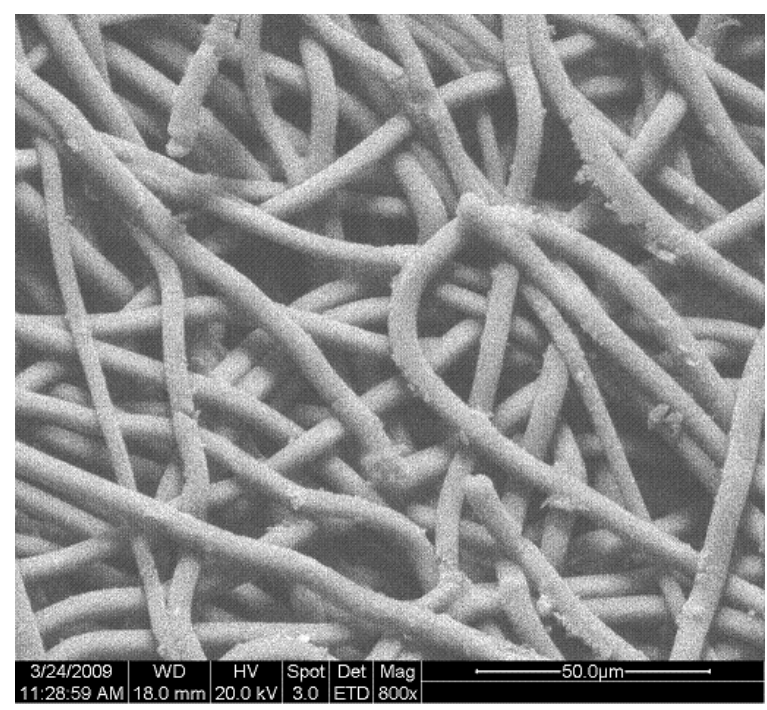

Figure 21 - SEM image of SPIN 6, albumen of PLGA electrospun construct (610X).

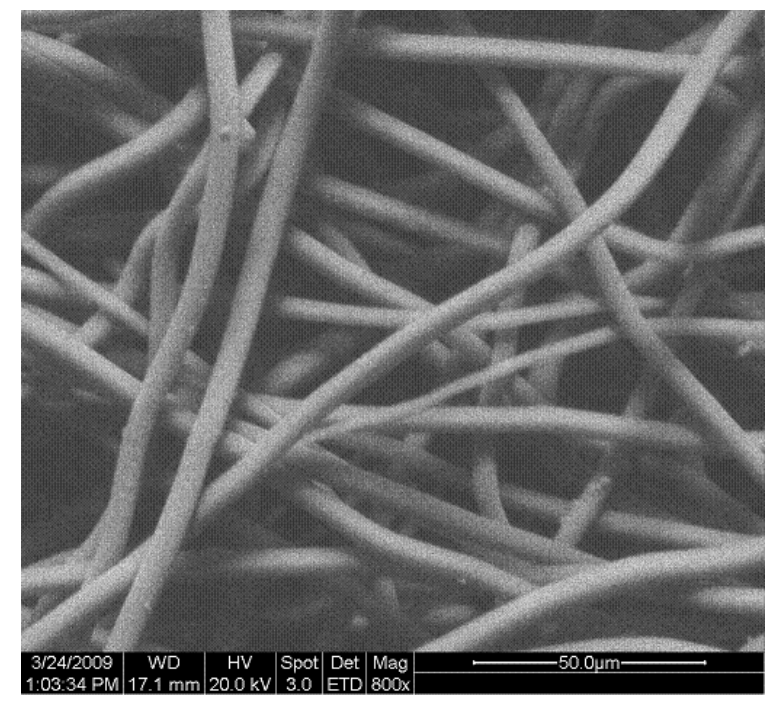

Figure 23 - SEM image of SPIN 7, ablumen of PLGA electrospun construct (800X). 


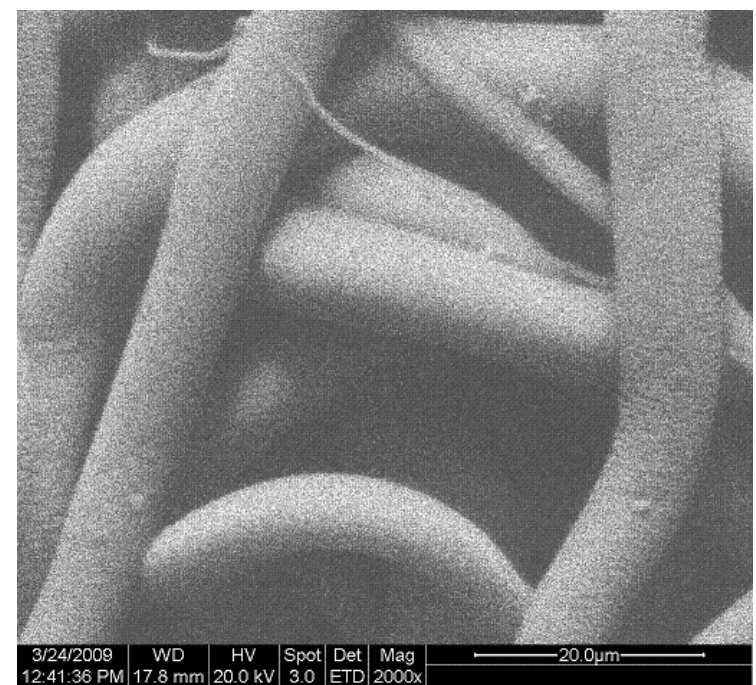

Figure 24 - SEM image of SPIN 8, lumen of PLGA electrospun construct (2000X).

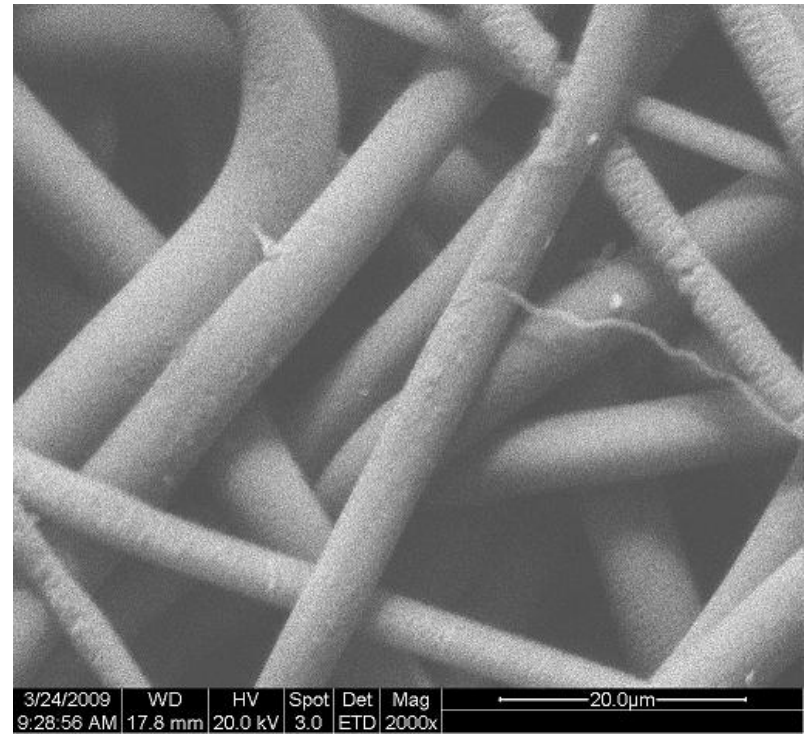

Figure 26 - SEM image of SPIN 9, lumen of PLGA electrospun construct (2000X).

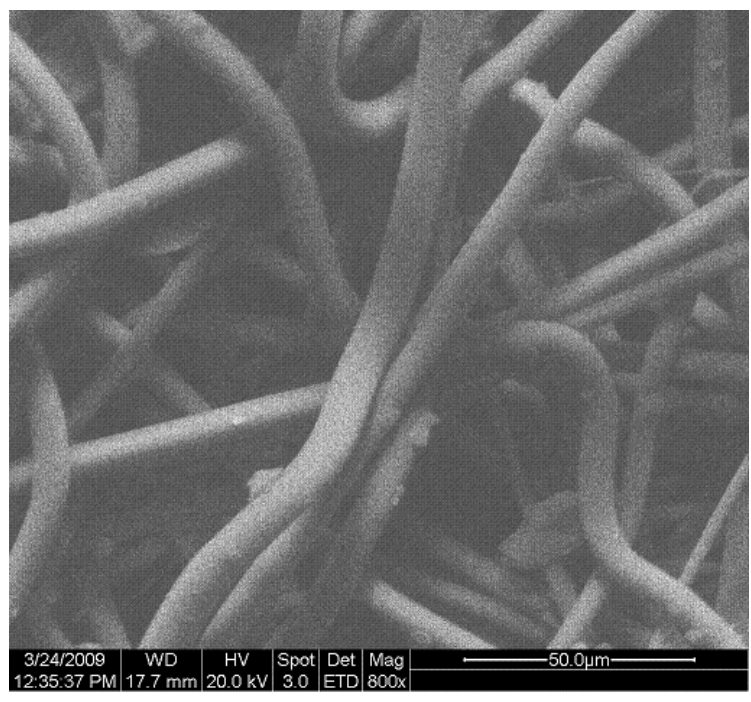

Figure 25 - SEM image of SPIN 8, ablumen of PLGA electrospun construct (800X).

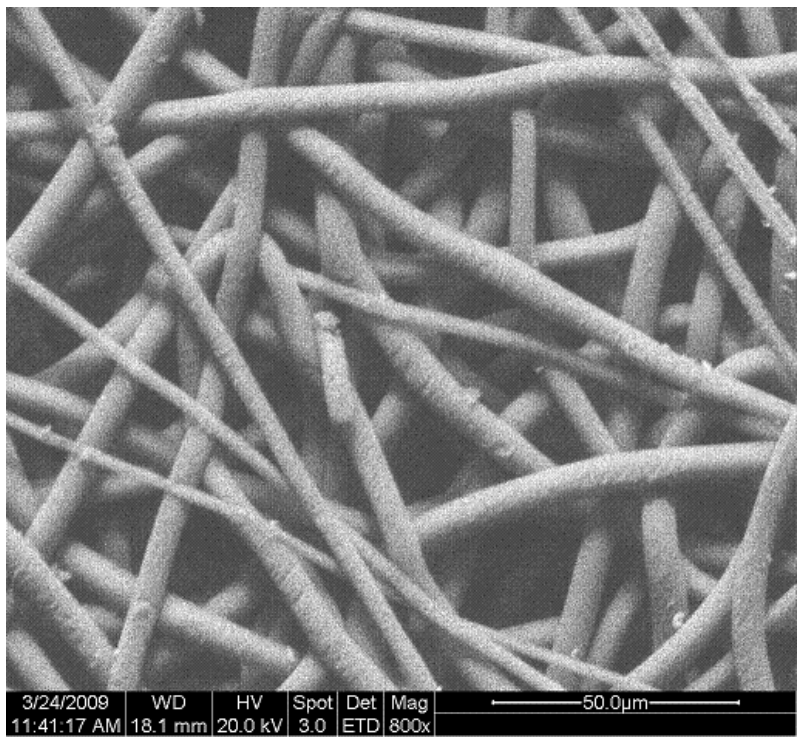

Figure 27 - SEM image of SPIN 9, ablumen of PLGA electrospun construct (800X). 


\subsubsection{Mechanical Testing}

Tensile properties of electrospun $15 \mathrm{wt} \%$ PLGA scaffolds prepared with different processing parameters are provided in Table VII. Stress strain plots and Young's modulus plots for all spins can be found in Appendix G.

\section{Table VII - Mechanical Properties of 15 wt $\%$ PLGA}

\begin{tabular}{c|ccc|ccc|} 
& \multicolumn{3}{|c|}{ Ultimate Tensile Strength } & \multicolumn{3}{c|}{ Young's Modulus } \\
\hline $\begin{array}{c}\text { Spin } \\
\text { Title }\end{array}$ & Circumferential & $\begin{array}{c}\text { Longitudinal } \\
\text { Set } 1\end{array}$ & $\begin{array}{c}\text { Longitudinal } \\
\text { Set 2 }\end{array}$ & Circumferential & $\begin{array}{c}\text { Longitudinal } \\
\text { Set 1 }\end{array}$ & $\begin{array}{c}\text { Longitudinal } \\
\text { Set 2 }\end{array}$ \\
\hline SPIN 6 & $4.29 \mathrm{MPa}$ & $3.37 \mathrm{MPa}$ & $3.23 \mathrm{MPa}$ & $39.4 \mathrm{MPa}$ & $82.9 \mathrm{MPa}$ & $114.6 \mathrm{MPa}$ \\
SPIN 7 & $4.89 \mathrm{MPa}$ & $5.27 \mathrm{MPa}$ & $\mathrm{NA}$ & $11.8 \mathrm{MPa}$ & $\mathrm{NA}$ & $\mathrm{NA}$ \\
SPIN 8 & $4.18 \mathrm{MPa}$ & $5.37 \mathrm{MPa}$ & $5.31 \mathrm{MPa}$ & $37.1 \mathrm{MPa}$ & $100.8 \mathrm{MPa}$ & $126.0 \mathrm{MPa}$ \\
SPIN 9 & $3.60 \mathrm{MPa}$ & $4.13 \mathrm{MPa}$ & $3.13 \mathrm{MPa}$ & $37.6 \mathrm{MPa}$ & $130.1 \mathrm{MPa}$ & $111.6 \mathrm{MPa}$
\end{tabular}

Missing data for SPIN 7 was due to operator error when the data acquisition system was exited before properly saving the data. Tensile strength and ultimate tensile strength for all spins, in both the longitudinal and radial directions, is within range for the native vessel (approximately 1.40 - 11.14 MPa [58]). Young's modulus for the human blood vessel has been reported at 1-3 MPa, however collagen alone has been reported to have a Young's modulus of approximately $45 \mathrm{MPa}$ [138]. Circumferential Young's modulus for all spins ranged from 37-39 MPa except for SPIN 7 in which case some data was lost and the results are skewed. Longitudinal ultimate tensile strength (UTS) and Young's modulus were higher than the circumferential values for all spins except for SPIN 6 whose radial UTS was greater than the 
longitudinal UTS. There were no observable trends between mechanical properties and fiber diameter, although an increase in stiffness with increased fiber diameter would be a logical conclusion. However, given the relatively small range of fiber diameters achieved for SPIN 6-9, it is reasonable that no trend would be apparent.

It is important to note that a common failure location of tensile test samples was at the test grips. This is expected due to the rectangular shape of the sample pieces. Also, deformation of samples is possible due to cutting and flattening of the pieces during sample preparation and loading into the Instron tester. This is especially true for radial samples where more deformation is necessary to load the sample properly.

\subsection{Discussion}

\subsubsection{Solvent Selection}

The importance of the solvent is to adequately bring the polymer resin into solution and to evaporate quickly when exposed to air during the electrospinning process. Evaporation of the solvent is necessary for the thinning of the fiber jet and the production of a mostly solid fiber upon collection. For this reason both THF and $\mathrm{CHCl}_{3}$, two highly volatile solvents, were considered. DMF was considered as it is often used in combination with THF and $\mathrm{CHCl}_{3}$. Although THF is slightly more volatile than $\mathrm{CHCl}_{3}$, the cost for THF compared to $\mathrm{CHCl}_{3}$ was much greater. An important aspect of the BVM model is cost effectiveness and scalability; therefore chloroform would be the optimum choice due to cost savings of $\$ 15.00-20.00$ compared to THF. For this reason DMF was disregarded as well, since chloroform has proven to be capable of producing electrospun constructs without the additional solvent in primary literature. This too will keep the cost low. In early experimental trials comparing electrospun 
PLGA in both THF and $\mathrm{CHCl}_{3}$, solutions of $\mathrm{CHCl}_{3}$ were the first to be successfully electrospun. This was due to greater viscosity of $\mathrm{CHCl}_{3}$ solutions compared to THF solutions of the same wt\% in PLGA. In conclusion, trial experiments showed no significant benefit for one solvent compared to the other; however, the early success with $\mathrm{CHCl}_{3}$ was promising. Selection of $\mathrm{CHCl}_{3}$ came down to the fact that previous work on the feasibility of electrospinning scaffolds for the $\mathrm{BVM}$ lab used $\mathrm{CHCl}_{3}$ for electrospinning $\mathrm{P}(\mathrm{LLA}-\mathrm{CL})$ solutions. This previous experience will be beneficial for comparison of electrospun constructs and troubleshooting as necessary. Future work could pursue chloroform combinations with DMF as often done in literature. Investigations would need to be conducted on the benefits to such combination verses no combination. In conclusion, $\mathrm{CHCl}_{3}$ is an acceptable solvent, based on cost, previous use, and volatility, to use for the investigation of electrospinning PLGA for the BVM.

\subsubsection{Solution Concentration}

The large droplet formation at the tip of the needle observed during SPIN 1 could be due to high flow rate $(6.1 \mathrm{ml} / \mathrm{hr})$ combined with a low solution concentration and/or a low voltage. However, multiple fiber jet formations coming from the large droplet would be indicative of a voltage that was too high. A disadvantage to the process was primarily the loss of polymer associated with polymer solution accumulating on the tip of the capillary. When the large droplet becomes unstable and falls from the capillary, the continuous fiber jet breaks which could potentially cause a layering effect where each new jet forms a sheet over the previous collected jet. However, this layering effect hypothesis was not investigated. SPIN 2 was more successful with a decrease in flow rate to $2.5 \mathrm{ml} / \mathrm{hr}$ and an increased solution concentration compared to SPIN 1. A mostly stable jet was formed for SPIN 2; however one fiber formed towards the ceiling. This could be due to residual charge or polymer in the containment chamber 
that was not removed prior to the beginning of the spin process. During SPIN 3, flow rate was slightly increased ( $3 \mathrm{ml} / \mathrm{hr}$ ) from SPIN 2. A successful jet was formed, however, the droplet elongated until it fell to the floor of the containment chamber. Although the jet formation appeared optimum and no additional jets formed, the wasted polymer during the spin is still a disadvantage. Again, polymer build up suggests a flow rate that is too high. However, adjusting flow rates during both SPIN 4 and SPIN 5 led to accumulation of polymer on the needle tip regardless of the flow rate with a slower accumulation evident with slower flow rates and faster accumulation at higher flow rates. Ruling out flow rate and applied voltage, solution viscosity is the only factor remaining that may be the primary source of large droplet accumulation.

Solution concentration is the most effective method of altering fiber diameter during electrospinning [85]. The high surface area per unit volume ratio achieved in electrospun micron and nano-fiber constructs can allow for enhanced cell proliferation, adhesion, and differentiation in culture. Since solution concentration is the dominate parameter leading to construct morphology, a solution concentration that is capable of producing continuous, non beaded fiber networks was the first step to building an electrospinning protocol for this thesis. From the results of this study, $15 \mathrm{wt} \%$ PLGA in $\mathrm{CHCl}_{3}$ (SPIN 4) solutions were determined optimum. This was the lowest concentration of PLGA in $\mathrm{CHCl}_{3}$ that led to continuous non-beaded fiber networks. Higher viscosity is known to favor the formation of non-beaded networks as also evident from the results of this study, as well as reports in literature [102]. Surface tension favors the formation of beads and reduced surface tension favors no beads. As mentioned previously, solution concentration inversely affects surface tension and therefore an increase in solution concentration should produce less bead defects. This is also evident from the results of SEM analysis. SPIN 5, $18 \mathrm{wt} \%$ solution, also produced non-beaded continuous fiber constructs. 
However, the higher the solution concentration the larger the fiber diameter by a linear relationship and therefore, in order to achieve the smallest fiber diameter with no bead defects, the $15 \mathrm{wt} \%$ solution was selected. Although the nano-fibers associated with beaded networks are ideal, the beads are disadvantageous to TEVG development because they decrease the much desired high surface per volume ratio achievable in fibrous electrospun constructs. Cellular attachment and migration is also hindered by such large defects [139]. Micron-size fibers are acceptable in that they are smaller than the endothelial cells they will interact with and therefore acceptable for the ongoing of this investigation.

\subsubsection{Optimizing Processing Parameters}

Electrospinning process parameters were optimized for a solution of $15 \mathrm{wt} \%$ PLGA in $\mathrm{CHCl}_{3}$ in order to determine a final electrospinning protocol for further investigation of scaffold endothelialization. Applied voltage and flow rate were the two parameters adjusted for processing optimization. Voltage and flow rate in combination with solution viscosity must be in balance for stable jet formation and consistent fiber morphologies. Comparisons between resultant fiber diameters and mechanical properties showed minor differences between constructs due to changes in applied voltage and flow rate. This suggests solution viscosity has the ultimate control over final construct morphology as often shown in literature. However, adjusting flow rate and applied voltage until a well balanced process is formed is optimum for process efficiency and repeatability.

Processing parameters in accordance with SPIN 6 will be used for all further investigations of PLGA. The smallest achievable fiber diameter was seen for SPIN 6 with an average fiber diameter of $5.49 \pm 0.93$ microns. Such fiber diameters are smaller than the 
endothelial cells they are intended to interact with and are acceptable for the continuation of this investigation. The variation in fiber diameter for SPIN 6 could be explained by the high humidity (47\%) during the spin. High humidity could hinder solvent evaporation which may affect fiber consistency. This trend is supported by results for SPIN 7 with an average fiber diameter of $5.85 \pm 0.64$ microns at only $40 \%$ humidity. The only difference between SPIN 6 and SPIN 7 besides humidity was the flow rate, suggesting flow rate may also affect the consistency of fiber formation. However, SPIN 8 with an average fiber diameter of $7.35 \pm 1.00$ microns was spun with $38 \%$ humidity and the same flow rate as SPIN 7 suggesting flow rate has minimal effect on consistency of fiber formation. The major difference between SPIN 7 and SPIN 8 was applied voltage, ultimately suggesting an unstable jet from a high voltage causes the most inconsistency in fiber diameters of electrospun constructs. Future studies should investigate the effects of a controlled environment (i.e. humidity and temperature) and the potential advantages to electrospun construct morphologies to confirm this hypothesis. It should be noted that standard deviation could also be a result of a non-ideal measurement system using Image J and the potential for inaccurate measurements due to operator inconsistency.

Processing parameters for SPIN 6 are advantageous for the final protocol for both time and efficiency purposes. The flow rate used for SPIN 6 was set to $5.5 \mathrm{ml} / \mathrm{hr}$ which allows for approximately 1 spin every half hour, thus corresponding to six, $4 \mathrm{~cm}$, scaffolds per hour. At 5.5 $\mathrm{ml} / \mathrm{hr}$ and $12,000 \mathrm{~V}$, a stable jet was formed from the droplet at the tip of the capillary during the electrospinning process. For all spins regardless of flow rate and applied voltage, extruded droplet formations on the tip of the needle were observed. This is disadvantageous since polymer is wasted during the process. Such occurrences suggest solution viscosity is controlling droplet formation and further investigations will be necessary to make the process more efficient. 
However, SPIN 6 formed a consistent stable jet and no polymer was wasted due to stray jet formations or random collection around the collector and containment chamber. Only minor amounts of stray polymer, as seen for all spins, was collected around the mandrel rotator cuffs. This was also consistent with the previous investigations of electrospun P(LLA-CL).

Finally, results of tensile testing showed electrospun $15 \mathrm{wt} \%$ PLGA scaffolds had similar tensile strength in both circumferential and longitudinal directions compared to the native vessel. Young's modulus in the circumferential direction was also comparable to that of native collagen.

In conclusion, a final protocol was developed for electrospinning PLGA for further investigations into a replacement scaffold for the BVM. The final protocol uses PLGA dissolved in $\mathrm{CHCl}_{3}$ at a $15 \mathrm{wt} \%$ concentration. All future spins will be electrospun at $5.5 \mathrm{ml} / \mathrm{hr}$ and 12,000 V with a gap distance of $25.4 \mathrm{~cm}$ and an $18 \mathrm{GA}$ blunt tip needle. This was the optimum protocol determined for $15 \mathrm{wt} \%$ PLGA solutions. PLGA scaffolds electrospun at the final protocol will be cultured in perfusion bioreactors, according to standard BVM practices, with human endothelial cells, to assess the formation of intimal cellular lining. 


\section{Chapter 4 - Evaluation of Electrospun PLGA for the BVM}

\subsection{Introduction}

The goal of this investigation is to evaluate cellular response of human umbilical vein endothelial cells (HUVECs) to electrospun $15 \mathrm{wt} \%$ PLGA constructs. The implementation of electrospun polymer scaffolds could be advantageous in optimizing the scalability and cost effectiveness of the BVM model. Electrospinning polymer scaffolds also allows for research expansion and scaffold optimization specifically for the BVM design. PLGA is a promising material for tissue engineering vascular scaffolds and has shown good EC and SMC responses under pulsatile perfusion bioreactor systems [127]. The following investigation will evaluate electrospun 15 wt\% PLGA scaffolds sodded with HUVECs and cultured under physiological conditions in a continuous-flow bioreactor system. The potential for HUVECs to be sodded onto a PLGA scaffold and the subsequent formation of a luminal EC lining will be evaluated by fluorescent microscopy and histological staining. Prior to cell culture, reactions of PLGA scaffolds to ethylene oxide gas sterilization, nutrient rich media, and BVM flow conditions will be investigated. The purpose of those investigations is to ensure that the integrity of electrospun PLGA scaffolds will be persevered during the culture process. All scaffolds used for this investigation are fabricated according to the final protocol developed in Chapter 3 of this thesis.

\subsection{Methods}

\subsubsection{PLGA Response to Media}

The purpose of this investigation was to monitor the effects of PLGA in static growth media and consequences of degradation by-products and to observe scaffold integrity under 
flow. Bioreactor media was made by combining $500 \mathrm{ml} \mathrm{M199,} 56 \mathrm{ml}$ fetal bovine serum (FBS), $0.6 \mathrm{ml}$ fungizone, $5 \mathrm{ml}$ penicillin/streptomycin, $5 \mathrm{ml} \mathrm{L}$-glutamine and $2.8 \mathrm{ml}$ HEPES buffer. Media contains phenol red, an indicator of $\mathrm{pH}$. This is the same type of media used for BVM cultivation. For static experiments, two small samples of $15 \mathrm{wt} \%$ PLGA scaffolds, from SPIN 4 and SPIN 7, were placed in a $45 \mathrm{ml} \mathrm{BD}$ conical tube with media. Samples in static media were placed in the large incubator at $37^{\circ} \mathrm{C}$.

For flow experiments, two $4 \mathrm{~cm}$ PLGA scaffolds from SPIN 4 and SPIN 7 were used for flow analysis in the BVM. Female leur to barb connectors were fitted to each end of the scaffolds and sutured on with 2-0 silk suture. Each PLGA scaffold with connectors was fitted into a BVM chamber by non-sterile methods. Enough media was added to each BVM chamber to cover the scaffold and approximately $40-45 \mathrm{ml}$ of media was added to each reservoir. Chambers were closed and placed in the large ShelLab incubator and hooked up to a peristaltic pump (

Figure 28). Flow was maintained through the lumen of the scaffolds at a flow rate of 15 RPM. All samples (static and flow) remained in the incubator until noticeable change occurred or after 3 days. Scaffold samples under flow conditions were dried and preserved in a desiccator for further analysis. The $\mathrm{pH}$ levels of both static and flow media was measured. 


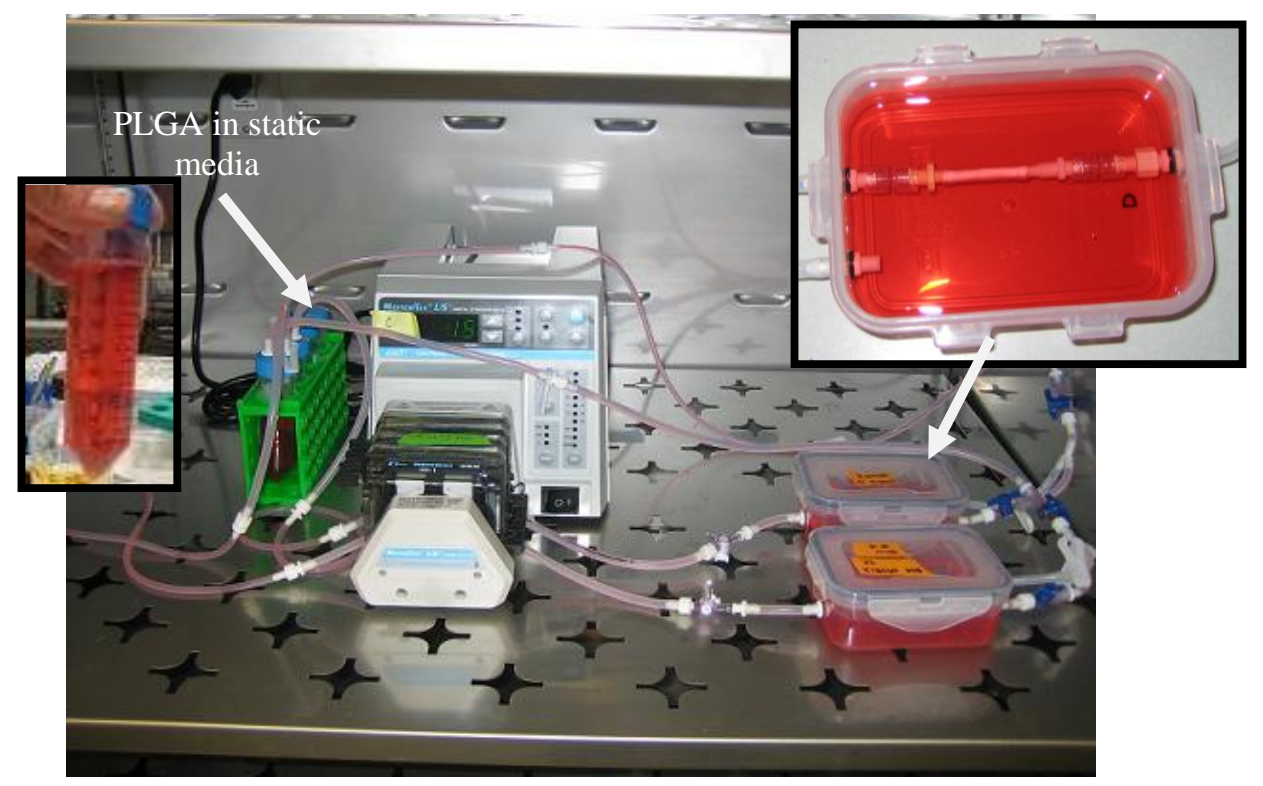

Figure 28 - The above picture shows both BVM chambers containing PLGA scaffolds hooked up to a peristaltic pump. PLGA samples in static media (left) are located in the back of the green tube rack.

\subsubsection{Morphological Analysis of Samples from Flow Experiments}

$15 \mathrm{wt} \%$ PLGA scaffolds from SPIN 4 and SPIN 7 investigated under low flow conditions in the BVM were analyzed using the SEM. Once dry, samples were removed from the desiccators and prepared according to section 3.2.2.1. Images were taken for qualitative purposes only.

\subsubsection{Cell Selection and Culture}

Human umbilical vein endothelial cells (HUVECs) were purchased from ScienCell Research Laboratories, Carlsbad, California and kept in vials in a liquid nitrogen dewar. Vials of cells were thawed and cultured according to standard practices. Endothelial cell media was made using $500 \mathrm{ml}$ endothelial cell basal medium, $5 \mathrm{ml}$ endothelial cell growth supplement, $5 \mathrm{ml}$ of penicillin/streptomycin solution and $25 \mathrm{ml}$ fetal bovine serum (Cat. No. 1001 from ScienCell). 
Cells were maintained in a small incubator at $37{ }^{\circ} \mathrm{C}$ and $5 \% \mathrm{CO}_{2}$. T225 BD cell culture flasks and endothelial cell media were used for the cell culture and expansion process.

\subsubsection{Preparation of Bioreactors and PLGA Scaffolds}

Four $15 \mathrm{wt} \%$ PLGA scaffolds were electrospun based on the final protocol developed in Chapter 3. Poly(D,L-lactide-co-glycolide) [75:25; PLGA; mol wt. 66,000 - 107,000; Cat. No. P1941] from Sigma Life Sciences and chloroform $\left(\mathrm{CHCl}_{3} ; 99.9 \%\right.$, extra dry, water <50 ppm; mol wt. 119.38; Cat. No. AC32682-0010_Acros Organics No.: 326820010) from Fisher Scientific were used for this experiment. Four 15 wt \% solutions of PLGA in $\mathrm{CHCl}_{3}$ were prepared according to Appendix B. Calculations to determine grams polymer necessary for a given wt\% solution were conducted based on $4 \mathrm{ml} \mathrm{CHCl}_{3}$. Each solution was electrospun according to Appendix C. Only $3 \mathrm{ml}$ of the $4 \mathrm{ml}$ solution was electrospun for each spin. Mandrel rotation was set to 6 and translation set to 3 for all spins. Flow rate was set to $5.5 \mathrm{ml} / \mathrm{hr}$, gap distance to $25.4 \mathrm{~cm}$, and applied voltage to $12,000 \mathrm{~V}$. An $18 \mathrm{GA}$ blunt needle was used for all spins. During each spin, environmental conditions remained stable at $35 \%$ humidity and 68.8 $\pm 1^{\circ} \mathrm{F}$ (normal conditions for that day). At the end of each spin, scaffolds were allowed to dry overnight in the desiccator. After that time, each spin was removed from the desiccator and cut into three $4 \mathrm{~cm}$ sections, placed on a glass stir rod and back in the desiccator for future use.

Five $4 \mathrm{~cm}$ sections of $15 \mathrm{wt} \%$ PLGA from the new spins, including one $4 \mathrm{~cm}$ section from SPIN 4 (from Chapter 3) were packaged and sent to the Cal Poly Veterinary Clinic to be Ethylene Oxide (EtO) gas sterilized for 24 hours. Bioreactor chambers, chamber lids, two-port reservoir lids, bioreactor tubing and fittings were also sterilized in the same manner. 


\subsubsection{BVM Bioreactor Set-up with HUVECs}

Bioreactor media and conditioning media were brought to $37^{\circ} \mathrm{C}$ in a water bath. All necessary sterile equipment was gathered. Using sterile techniques, 4 sterilized scaffolds ( 3 new and one from SPIN 4) were sutured onto sterile luer lock fittings with silk suture and attached to the inside of the sterile bioreactor chambers. PLGA scaffolds were too small for the BVM and additional sterile tubing was used to connect scaffolds in place. Typically a denucleation step is necessary to remove air from ePTFE scaffolds prior to cell sodding, however this step requires prolonged static conditioning which should be avoided with spun PLGA due to previous results from section 4.2.1 (PLGA Response to Media). Enough media was added to each BVM chamber to completely submerge the scaffold (Figure 29).

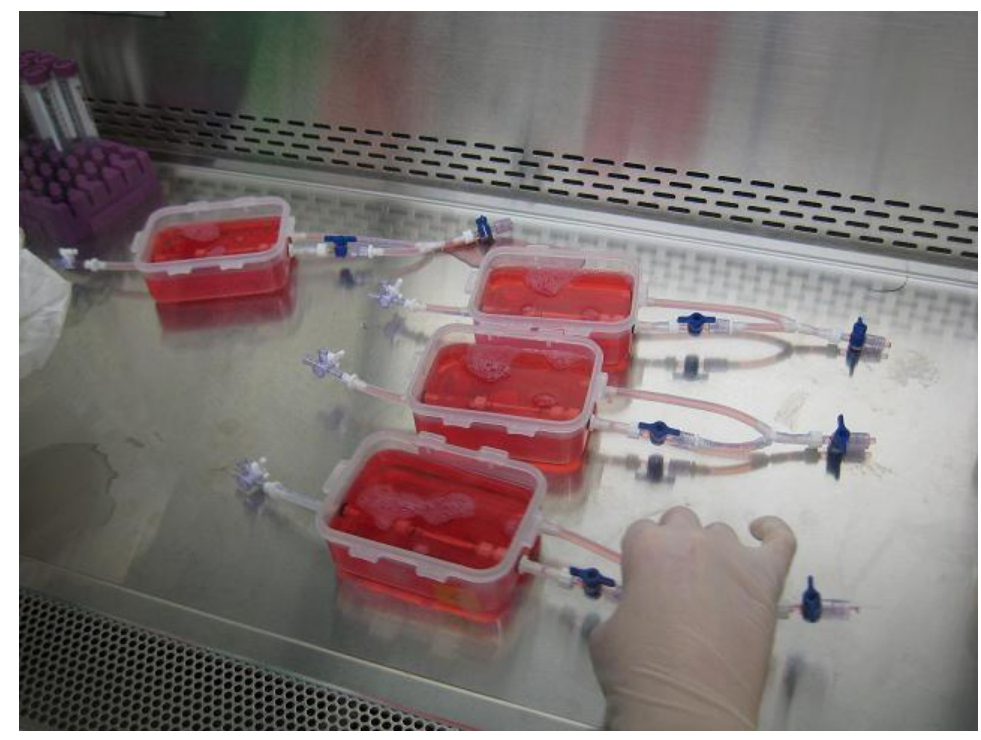

Figure 29 - Preparation of BVM chambers for cell sodding in a sterile environment. 
Each PLGA scaffold was actively syringe-primed with conditioning media by injecting media through the lumen of the scaffold until all the air was removed. The luminal outlet was then closed and media was transmurally forced through the pores of the PLGA scaffold.

Bioreactors with conditioning media were placed in a large ShelLab incubator and hooked up to a peristaltic pump. Bioreactors were further primed with one hour of flow with conditioning media. At the end of the conditioning process, each bioreactor was removed from the incubator and placed back in the sterile hood for cell sodding. Each scaffold was sodded with approximately 1 million HUVECs per $\mathrm{cm}^{2}$ (approximately 7 million cells per scaffold) using sterile techniques. Cells were pressure sodded and chased with $5 \mathrm{ml}$ of media. After sodding, media reservoirs were replaced with endothelial cell media. Bioreactors with the new media were again set up in the large ShelLab incubator and connected to a peristaltic pump (Figure 30). The incubator was maintained at $37{ }^{\circ} \mathrm{C}$ and $5 \% \mathrm{CO}_{2}$.

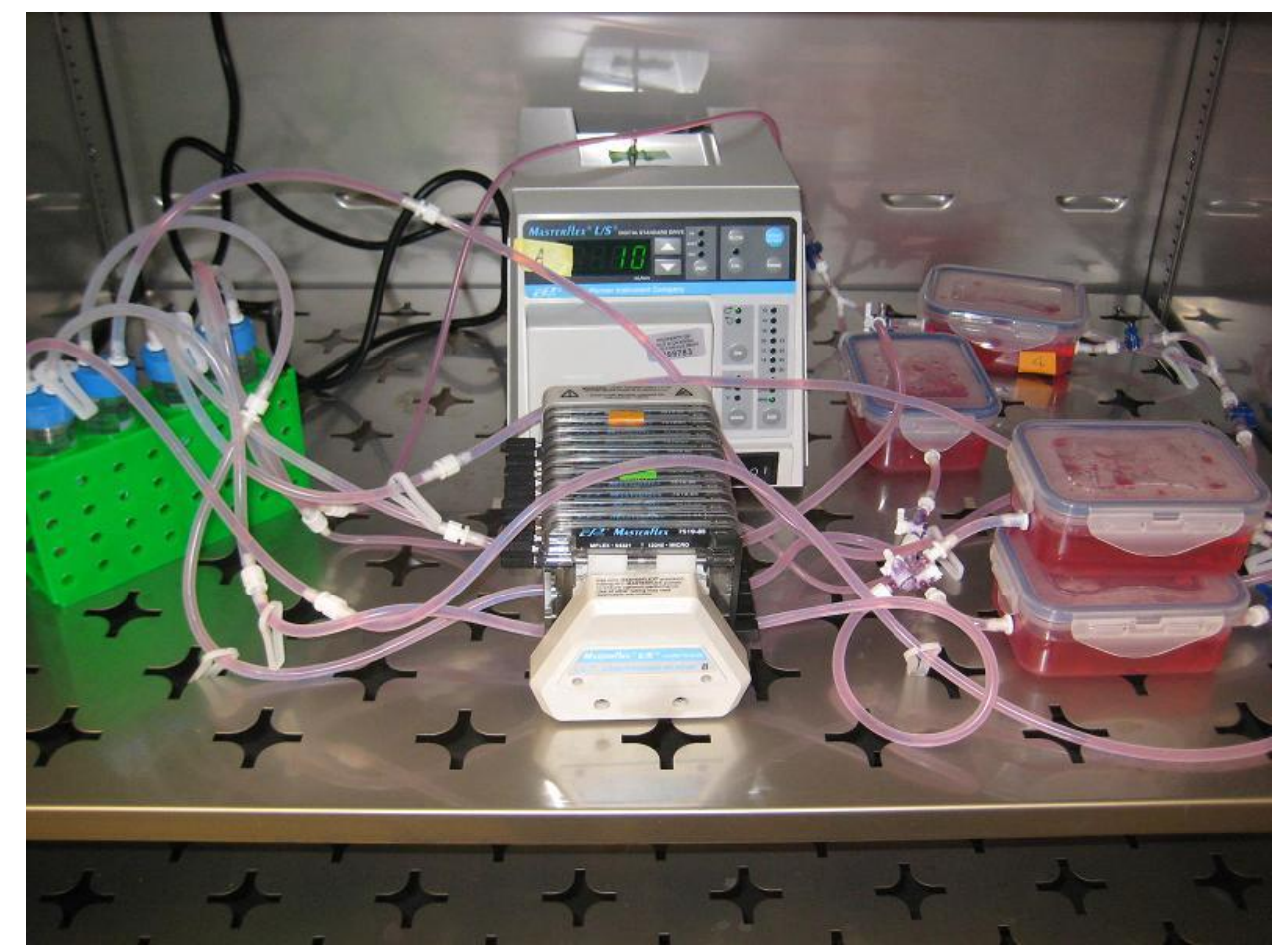

Figure 30 - Final setup of BVMs in incubator and hooked up to peristaltic pump. 
Once bioreactor setup was complete and cells were sodded, the luminal outlet remained closed and transmural flow was initiated for 5 minutes at 10 RPM. Generally this step is conducted for one hour to aid cellular attachment to scaffolds by pushing cells into the pores. However, due to the highly porous nature of the electrospun PLGA scaffolds and the limited resistance experienced during pressure sodding, there was concern that too much prolonged pressure would force the cells to penetrate too deeply into the scaffold. Following transmural flow, the pump was turned off for 20 minutes to allow time for cells to attach to the luminal surface of the scaffolds. The luminal outlet port was opened and flow was set to 10 RPM for a few hours and then ramped up to 25 RPM and later to 55 RPM. After 24 hours, one scaffold was harvested and the flow rate was set to 75 RPM for the remaining BVMs. After 3 days in the incubator, a second scaffold was harvested and flow rate was set to 90 RPM for the remaining two scaffolds. After 6 days in the incubator, the remaining two scaffolds were harvested.

\subsubsection{Harvesting of PLGA Scaffolds with HUVECs}

Scaffold harvesting was conducted with the removal of each BVM from the incubator according to the following protocol. All valves were closed on the bioreactor and the BVM chamber, including media reservoir, was removed from the incubator to a non-sterile work station. A $15 \mathrm{ml} \mathrm{VWR} \mathrm{conical} \mathrm{tube} \mathrm{was} \mathrm{labeled} \mathrm{appropriately} \mathrm{and} \mathrm{filled} \mathrm{with} \mathrm{approximately} \mathrm{10-}$ $12 \mathrm{ml}$ of histochoice fixative. Each HUVEC sodded PLGA scaffold was removed from the chamber and barb fittings using a surgical scalpel \#11. Each scaffold was then transferred to one of the prepared histochoice tubes, proximal side down, and set aside for future use. Residual PLGA was removed from the barb fittings and thrown away. Media was disposed of and the bioreactor, tubing, and fittings were cleaned with hot water. 


\subsubsection{Fluorescent Microscopy}

Bis-benzimide (BBI, Em $\lambda 460 \mathrm{~nm}$ ) was the stain used for analysis of HUVECs on electrospun PLGA scaffolds. In order to maintain the integrity of the BBI stain, the following procedures were conducted with minimum exposure to light. Twenty-five ml of Milli-Q (nonsterile) was placed in a $50 \mathrm{ml}$ BD conical tube. The tube was covered from the base to the lid with aluminum foil. Twenty-five $\mu \mathrm{BBI}$ was added to the tube containing Milli-Q and shaken vigorously. Four $15 \mathrm{ml}$ VWR conical tubes were wrapped in aluminum foil. To each $15 \mathrm{ml}$ tube, approximately $6 \mathrm{ml} \mathrm{BBI} \mathrm{solution} \mathrm{was} \mathrm{added.} \mathrm{PLGA} \mathrm{scaffolds} \mathrm{were} \mathrm{removed} \mathrm{from}$ histochoice and prepared for BBI one at a time. Each scaffold was cut into three samples; one cross section and two longitudinal sections. Approximately 1/3 of each scaffold on the distal end was cut for the cross sectional examination. The remaining $2 / 3$ of the scaffold was cut longitudinally to produce two samples for viewing the lumen of the scaffold. The most proximal end of each section was notched with scissors to maintain orientation when imaging. The three sections of each scaffold were placed in one of the $15 \mathrm{ml}$ tubes containing BBI stain. All samples remained in BBI for a minimum of 20 minutes and a maximum of 48 hours. The longer soaking time was found to more brightly stain the cells for better imaging. Images were acquired with an Olympus fluorescent microscope.

\subsubsection{Histology}

Histology was performed on all cross-sectional samples for all harvested PLGA scaffolds. The objective of histological analysis was to determine depth of cell penetration and presence of an endothelial cell lining. From primary literature there was some concern as to whether PLGA electrospun scaffolds would maintain integrity during processing due to the use 
of organic solvents. However, processing was conducted according to traditional methods. All samples were processed conventionally, embedded in paraffin wax, sectioned at $6 \mu \mathrm{m}$, then mounted on slides and stained with haematoxylin and eosin (H\&E).

\subsubsection{SEM Analysis}

HUVEC sodded PLGA scaffolds were also examined with the SEM. Cultured PLGA longitudinal sections previously used for BBI staining were dried in the desiccator. One end of each longitudinal section was cut for cross sectional examination. All sections were sputter coated in gold and analyzed.

\subsection{Results}

\subsubsection{PLGA Response to Media}

After 24 hours in static media at $37^{\circ} \mathrm{C}, 15 \mathrm{wt} \%$ PLGA scaffolds turned the media bright yellow, indicating a highly acidic change from normal $\mathrm{pH}$ to a measured $\mathrm{pH}$ of 4.82 (Figure 31 ). After one day, 15 wt\% PLGA samples under flow conditions appeared unchanged and the media was a normal color. After 3 days in the incubator under flow conditions, BVM media reservoirs became too low, allowing air into the tubes that stopped the continuous flow. Consequently the test was terminated. The $\mathrm{pH}$ of media in both chambers was measured. A pH of 8.76 for SPIN 4 and a $\mathrm{pH}$ of 7.6 for SPIN 7 were recorded. No observable change in scaffold integrity was observed. Degradation by-products did not cause an increase in acidity of the growth media. It is necessary to maintain normal physiological $\mathrm{pH}$ when tissue engineering to maintain cell viability. Reasons for no change in acidity for media in the BVM chambers could be due to the higher ratio of media to degradation by-products and the addition of gas permeable tubing. 


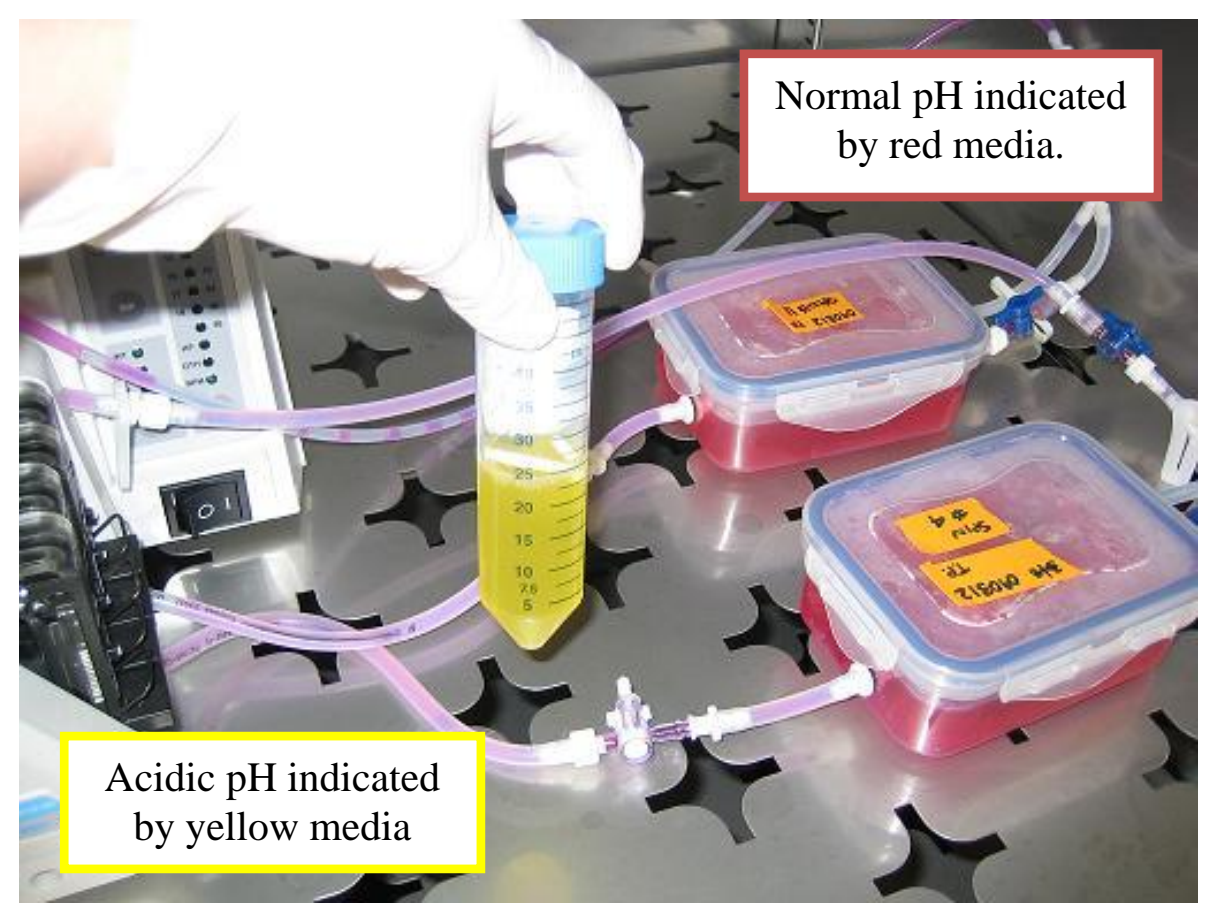

Figure 31 - Image of media color changes due to acidic by-products of PLGA in static media.

\subsubsection{Morphological SEM Analysis of PLGA under Flow}

Results of SEM analysis show several points where fibers are broken or cracked (Figure 32). Possible occurrence of breakage could be due to lack of scaffold integrity under low flow and low shear conditions. However, it is possible that fiber breakage was a result of drying the wet scaffolds in a desiccator immediately after removal. It was observed that samples became cracked and fragile after drying in the desiccator prior to SEM preparation and analysis. 


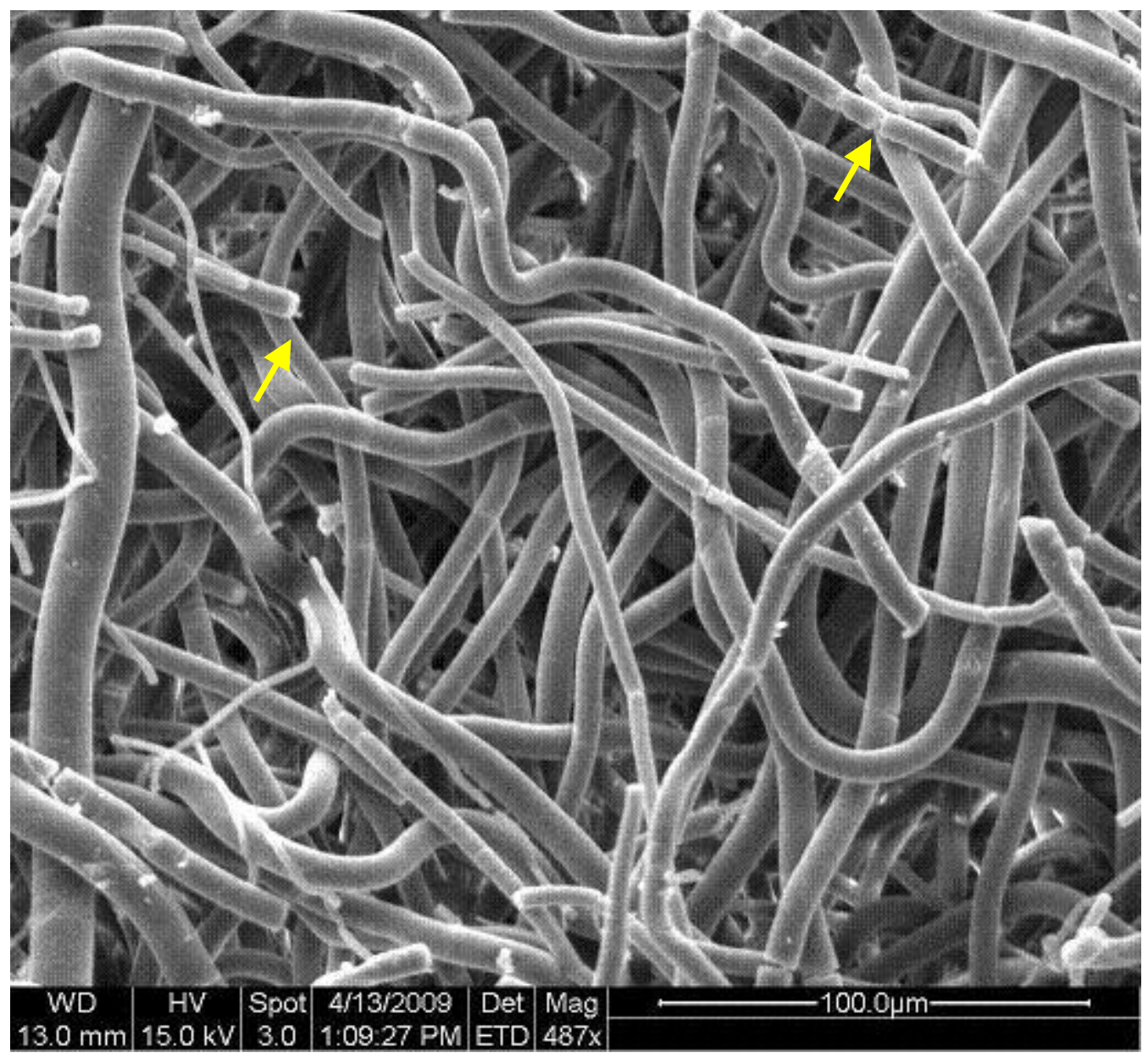

Figure 32 - The above SEM image was taken of the lumen of a PLGA scaffold after 72 hours of luminal flow. The image shows several broken and cracked fibers $(487 \mathrm{X})$.

\subsubsection{Preparation of Bioreactors and PLGA Scaffolds}

Electrospun PLGA scaffolds were successfully sterilized by EtO gas sterilization.

However, scaffolds appeared squished from the process due to unknown causes and some scaffolds were more squished than others (Figure 33). Since gas sterilization is conducted at the Cal Poly Vet Clinic, the process could not be monitored to determine cause of the deformed scaffolds. Although squished, the scaffolds were relatively easy to open to expose the lumen for fitting the barb connectors. For future sterilization, PLGA scaffolds should be pre-fitted to barb 
connectors to keep the lumen open and to minimize the steps necessitating a sterile environment. It was also observed that PLGA scaffolds were stiffer after gas sterilization. The increase in stiffness made scaffold insertion into the BVM chambers more difficult. Each PLGA scaffold had been cut to $4 \mathrm{~cm}$ which should have allowed for easy insertion with the appropriate female and male fittings. However, at that length, a little flexibility in the scaffold material is necessary to connect the scaffold into the BVM chamber. In order to fit the PLGA scaffolds into the chambers, custom sized tubing was used to allow for the flex necessary to the secure the scaffold into place. The adjustable tubing was fitted between the female luer to barb fitting and the inlet or outlet port fitting.
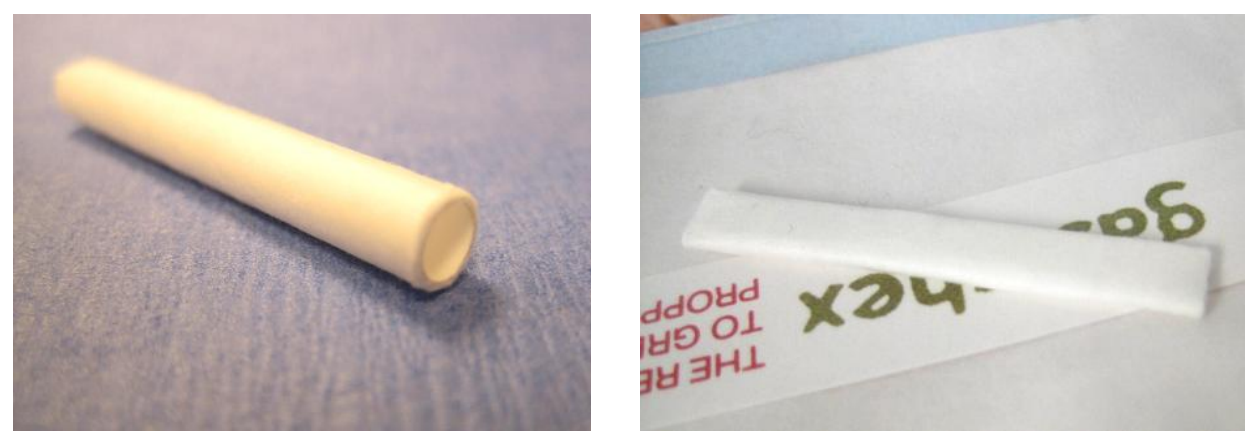

Figure 33 - Original electrospun scaffold is shown on the left. After gas sterilization the scaffold was severely flattened (right).

\subsubsection{Fluorescent Microscopy}

The following table is a summary table of each HUVEC sodded and cultured PLGA scaffold that includes culture duration and flow rate upon removal. A naming scheme was attributed to each scaffold based on time of removal for clarity and is provided in the table (Table VIII). 
Table VIII - Summary Table of HUVEC Sodded PLGA Scaffolds

\begin{tabular}{c|c|c} 
Title & $\begin{array}{c}\text { Culture } \\
\text { Duration }\end{array}$ & Flow Rate \\
\hline BVM01 & 24 hrs & 15 RPM \\
BVM02 & 3 days & 75 RPM \\
BVM03 & 6 days & 90 RPM \\
BVM04 & 6 days & 90 RPM
\end{tabular}

The first construct to be removed from the incubator and fixed in histochoice was a final protocol 15 wt\% PLGA scaffold. From this point forward the scaffold will be referred to as BVM01. Total duration of culture was 24 hours and maximum flow reached was 15 RPM. BBI images of BVM01 showed definite appearance of cells on the lumen of the scaffold (Figure 34). One image is in black and white and the other in color. This is because the color camera was not installed during initial imaging. Cells look balled up as typically expected for such a short culture time. The scaffold was difficult to image given the curved nature of the scaffold. Unclear images can be attributed to inexperience of the operator with BBI and fluorescent microscopy.

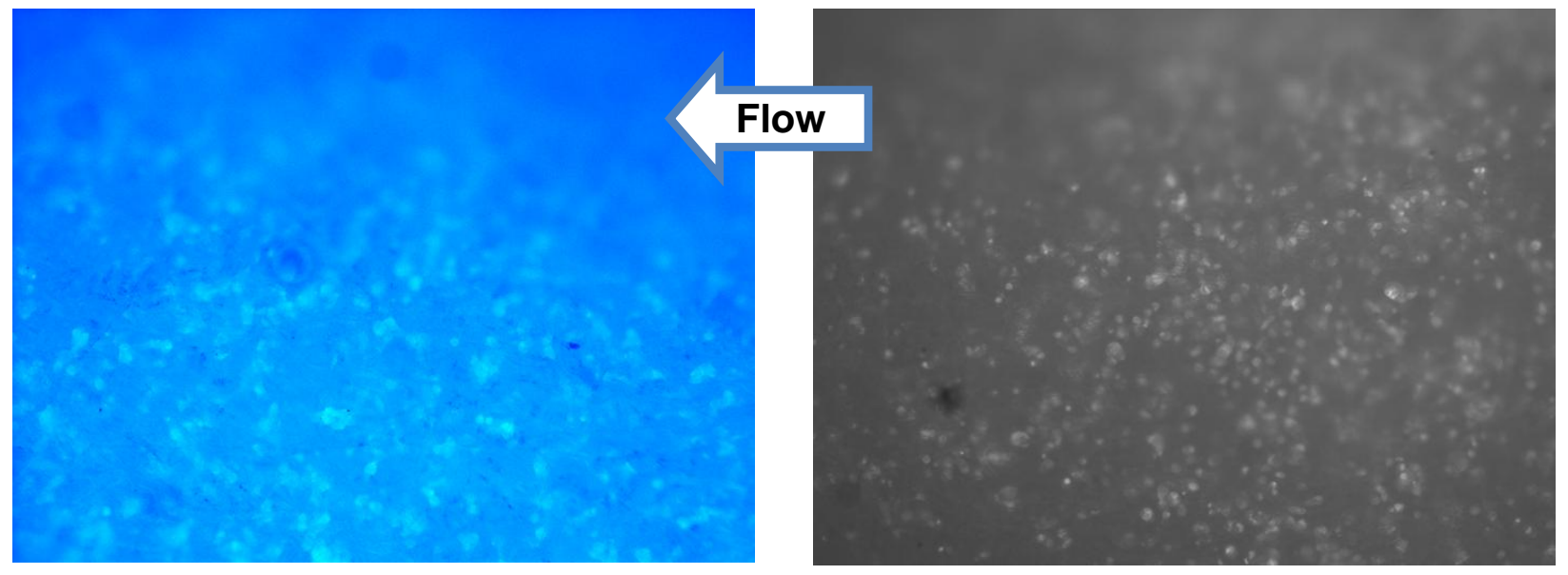

Figure 34 - Images of BVM01 cultured with HUVECS for 24 hours under flow at 15 RPM. Both proximal (right) and distal (left) ends of the graph have visible cells (10X). 
The second PLGA construct to be removed from the incubator and fixed in histochoice was also a PLGA scaffold produced at the final protocol. From this point forward the scaffold will be referred to as BVM02. Total duration of culture was 72 hours and maximum flow reached was 75 RPM. Cells can be observed on BVM02 from the distal to proximal end of the scaffold (Figure 35). It appears that cells have penetrated deeper into the pores compared to BVM01. Images for all scaffolds were taken at 10X and BVM02 cells seem slightly smaller than BVM01, alluding to the deep penetration hypothesis.

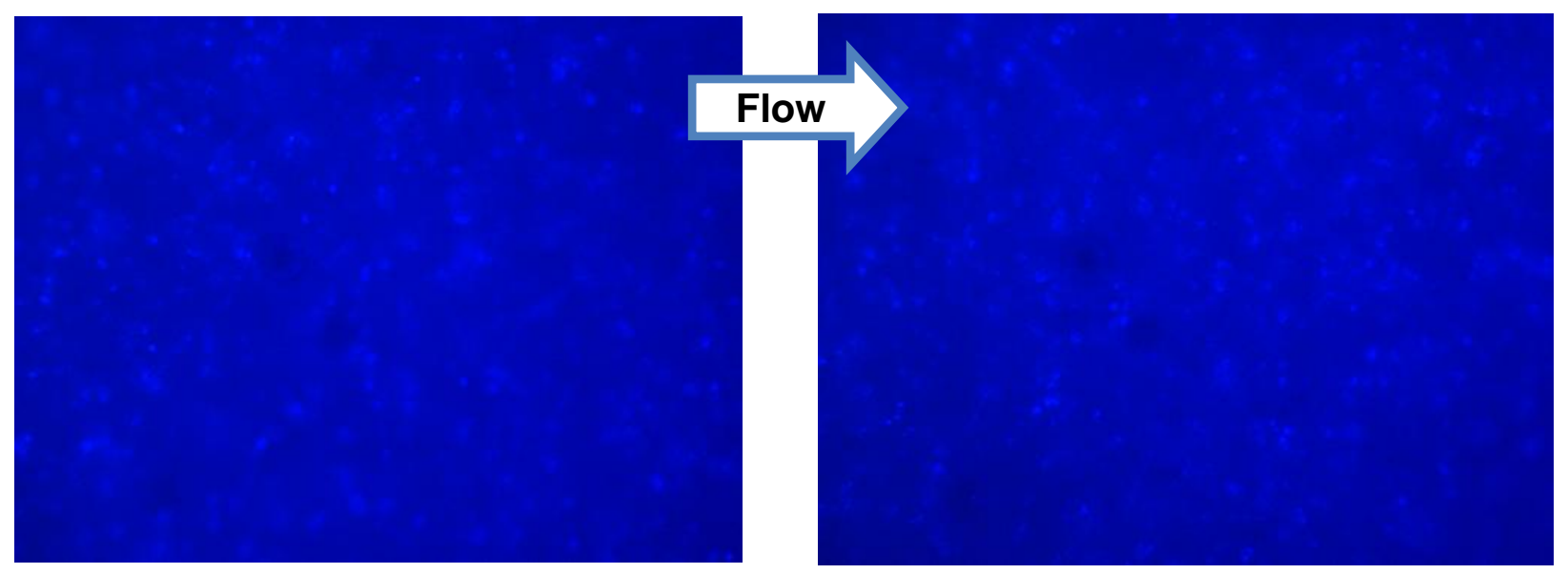

Figure 35 - Images from BVM02 cultured with HUVECs for 3 days. Cells are evident at both distal (right) and proximal (left) areas of the scaffold (10X).

The third and fourth PLGA constructs to be removed from the incubator and fixed in histochoice were a section from SPIN 4 and a final protocol PLGA construct. From this point forward SPIN 4 will be referred to as BVM04 and the new construct will be referred to as BVM03. Both BVM03 and BVM04 were cultured for 6 days and at a maximum flow rate of 90 RPM. There is little difference between the number of cells appearing on BVM03 (Figure 36) and BVM 04 (Figure 37) compared to BVM02 (Figure 35). However cells do look smaller than 
those present on BVM01, again suggesting cells are penetrating deeper into the porous PLGA constructs. The first round of imaging for BVM03 and BVM04 was difficult to obtain clear images therefore new BBI was made and scaffolds were allowed to soak for at least 24 hours before viewing. Images were much brighter after soaking the additional time in new BBI solution.

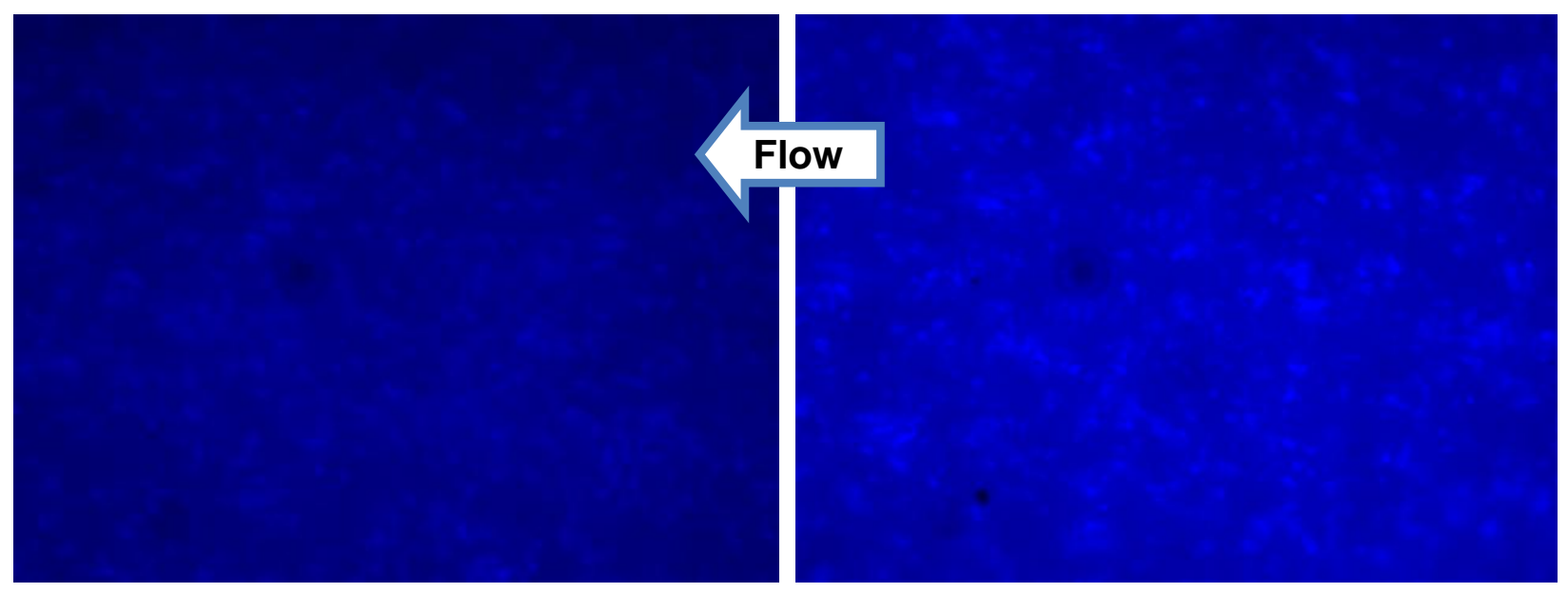

Figure 36 - Images of BVM03 show cells present for both distal (left) and proximal (right) regions of the scaffold.

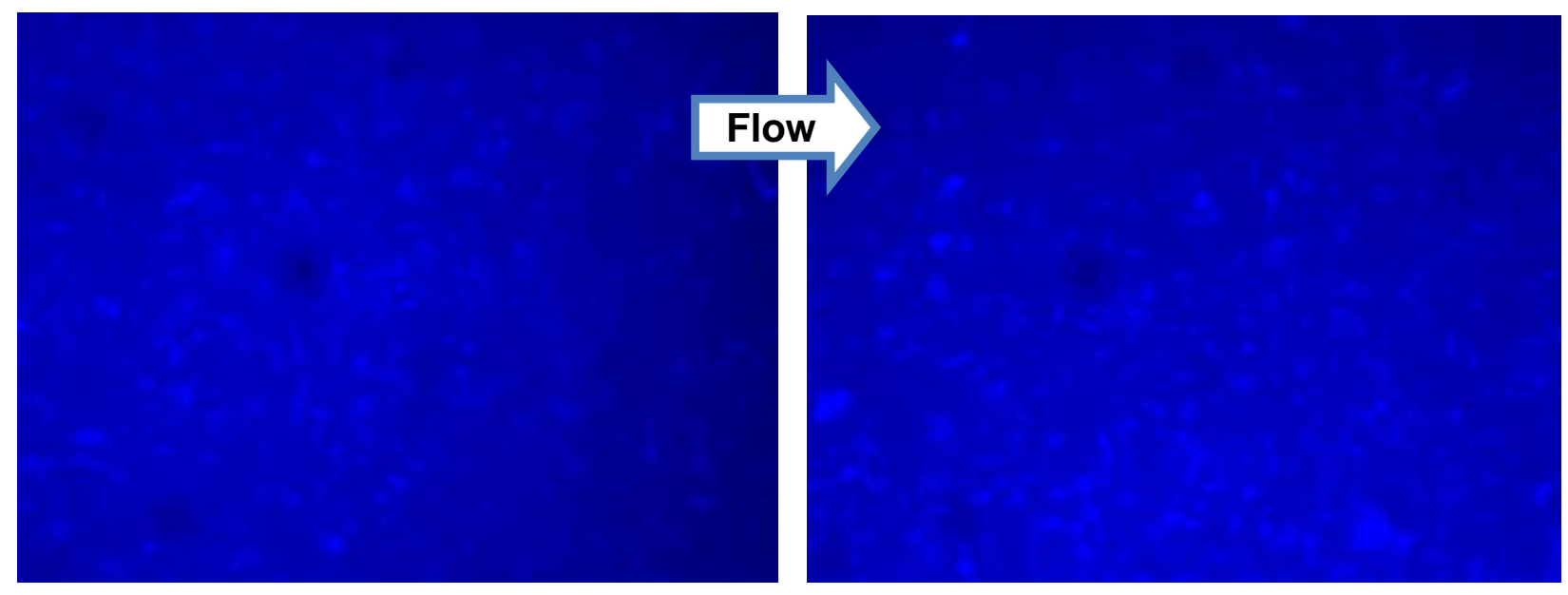

Figure 37 - Images of BVM04, both proximal (left) and distal (right), show several cells present. 
The larger image included below was taken from BVM03 (distal) (Figure 38). The image clearly shows fibers interweaving over and around cells, further suggesting the deeper penetration of cells into the PLGA scaffolds. Histological analysis was conducted in order to determine depth of cell penetration and or presence of an intimal cellular lining.

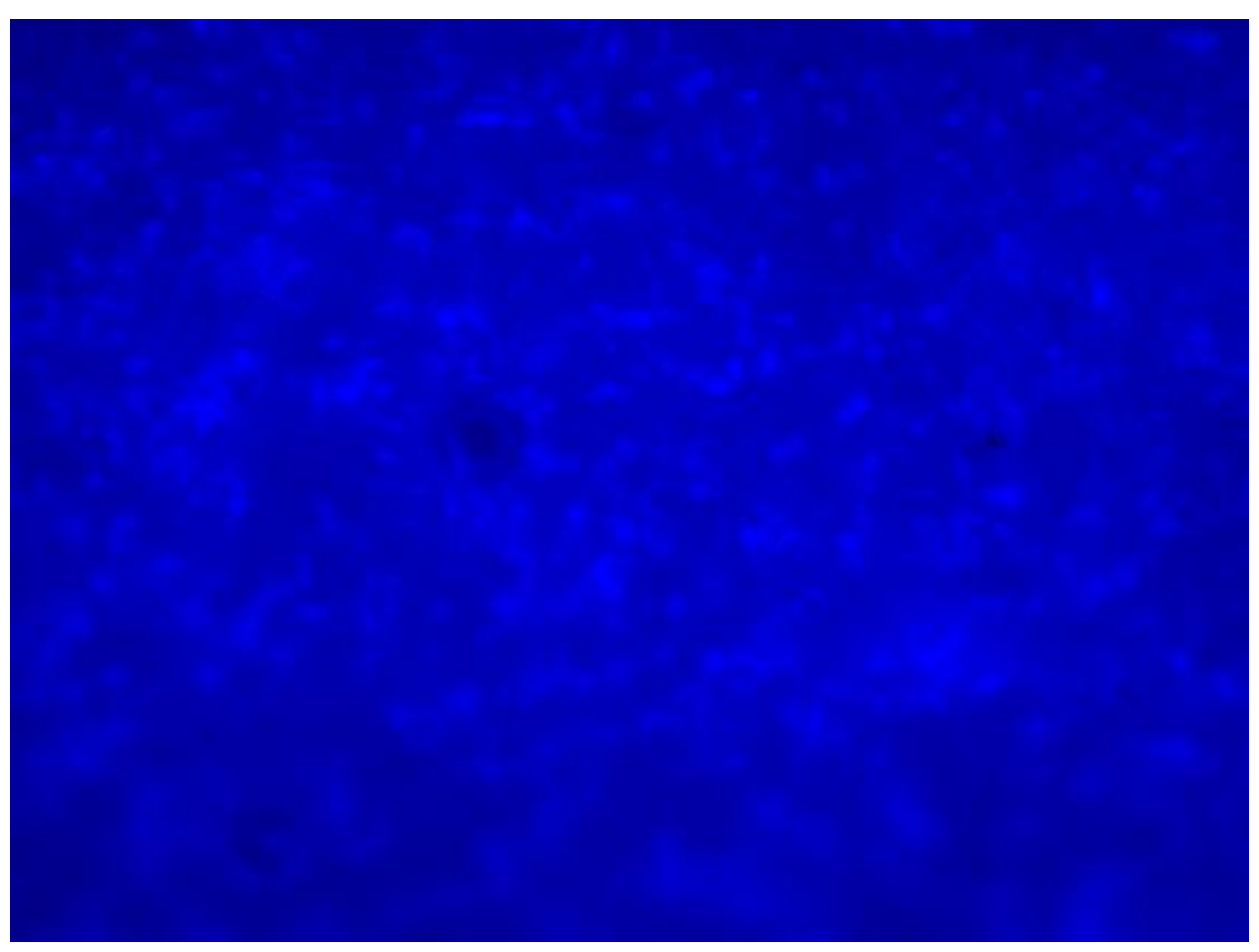

Figure 38 - The above image from BVM03 shows the fibrous nature of the PLGA scaffolds. Cells appear to be under some fibers.

\subsubsection{Histology}

As mentioned previously, there was some concern as to the integrity of HUVEC sodded PLGA scaffolds when processed for histology with organic solvents. After conventional processing, samples were removed and were noticeably smaller than their original size prior to processing. Also, PLGA samples were squished and it was difficult to re-open the lumen 
without damaging the structure of the samples. Regardless, BVM01-04 were successfully embedded in wax. During microtone slicing of samples, BVM02 (75 RPM, 72 hrs) fell out of the wax mold and staining of that sample was unable to be performed. Histological imaging of BVM01, 03, and 04 shows clear evidence of damage to the scaffolds due to processing. Significant damage occurred for BVM03 (90 RPM, 6 days). Images showed scattered cells at various locations in the polymer (Figure 39). The shape observed was no longer an open lumen but a semi deformed circle.
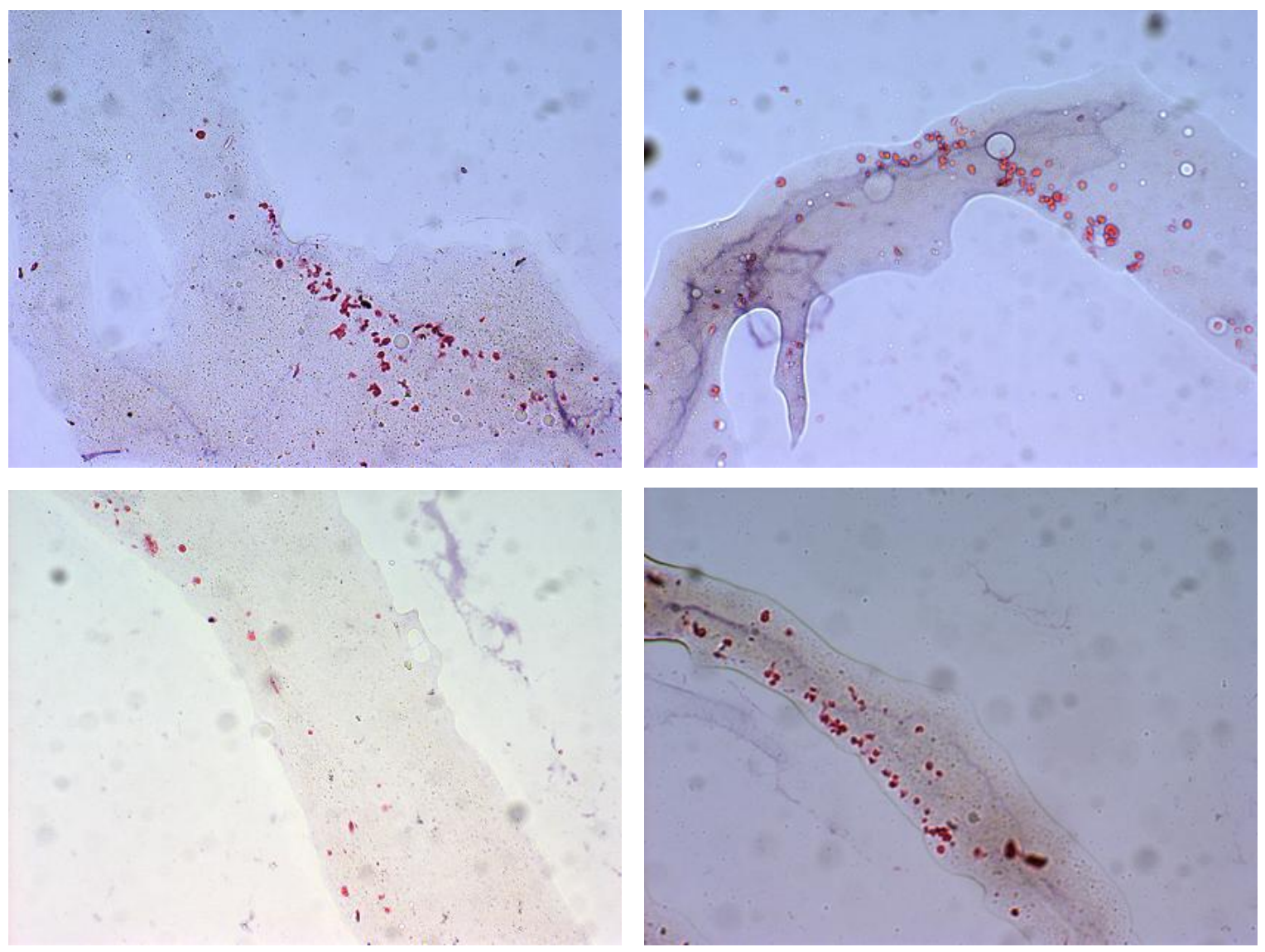

Figure 39 - Histology images of BVM03 shows cluster of cells and a damaged sample.

Deformation was also observed for BVM04 (90 RPM, 6 days). The lumen of the sample was closed during wax embedding. Images show a closed vessel with a cellular line following 
the center of the vessel (Figure 40). Even still the scaffold is clearly deformed and no conclusion can be drawn as to the exact location of the cells.
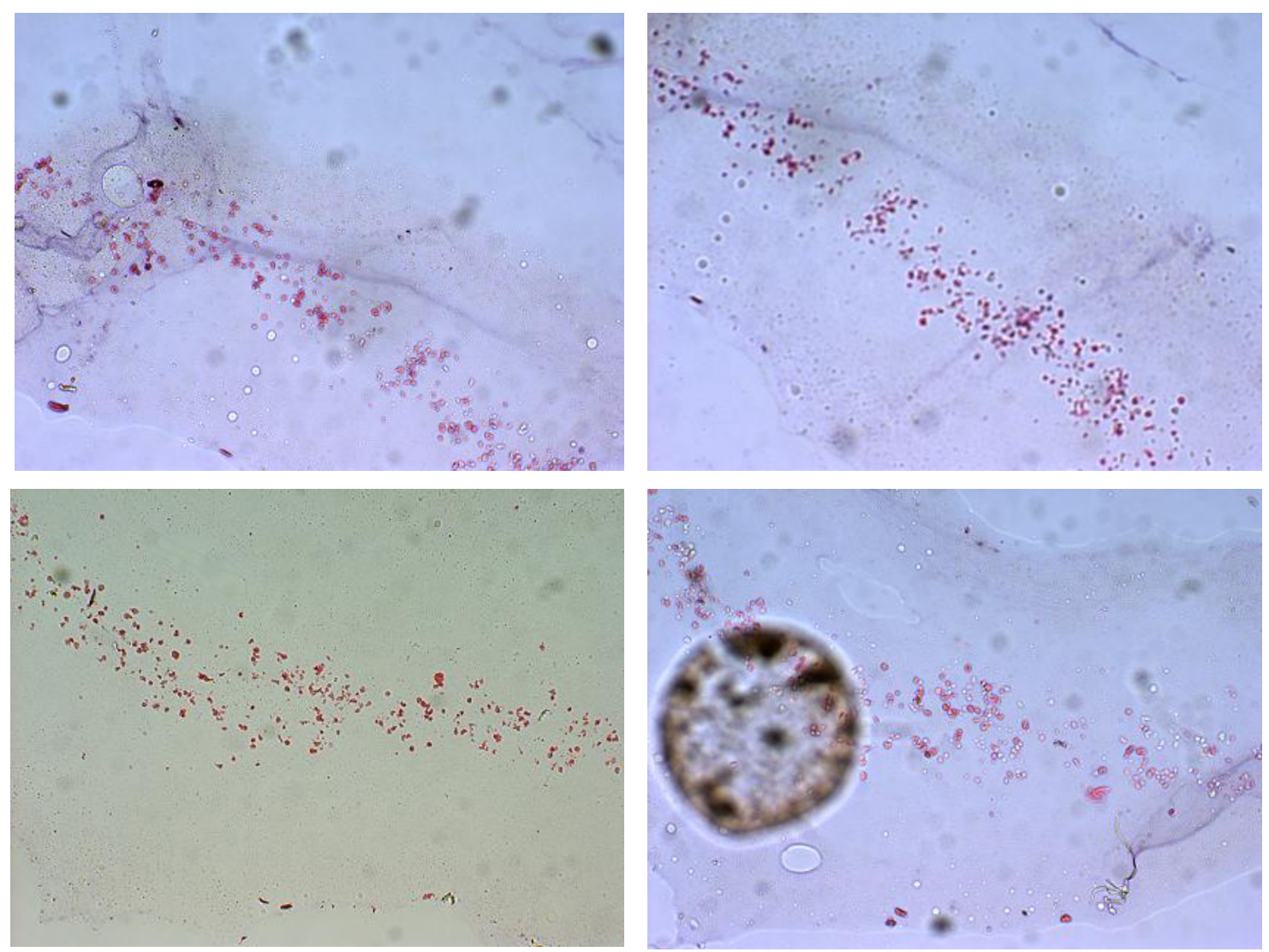

Figure 40 - Histology shows the lumen of BVM04 was closed during processing. A cellular lining is seen down the center of the vessel.

Unlike BVM03 and BVM04, the lumen of BVM01 remained open and images of the cellular lining were obtained. Still there is evidence of severe deformation of the scaffold from processing. No conclusive evidence exists as to the penetration of cells given the severity of scaffold deformation and the potential disruption of the cellular lining. It is possible that the cellular lining acted as a protective barrier for the polymer on the lumen, allowing for the images below. 

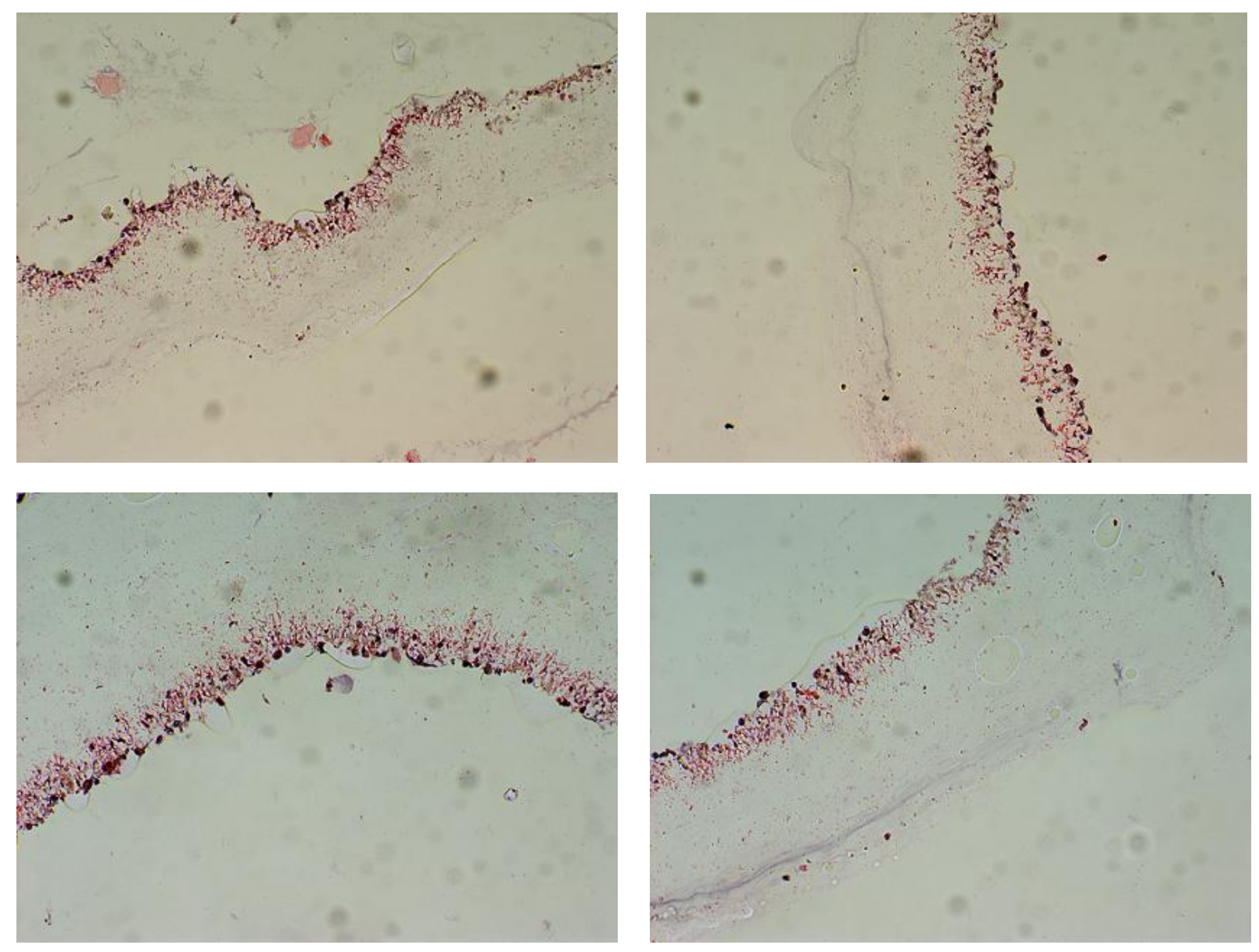

Figure 41 - Histology images of BVM01 show a cellular lining on the lumen of the vessel.

Unfortunately no conclusion can be drawn from histological analysis of HUVEC sodded PLGA scaffolds. Scaffolds were highly deformed after conventional histological processing of the PLGA scaffolds suggesting a new process or a modified process needs to be investigated.

\subsubsection{SEM Analysis}

To further investigate an intimal lining and the depth of cellular penetration for HUVEC sodded PLGA scaffolds SEM analysis was employed. Cells were apparent on the lumen of BVM01 (15 RPM, 24 hrs) [Figure 42]. The balled-up morphology of the cells is expected for such a short culture time point, however evidence of cell spreading can be seen (Figure 42). 


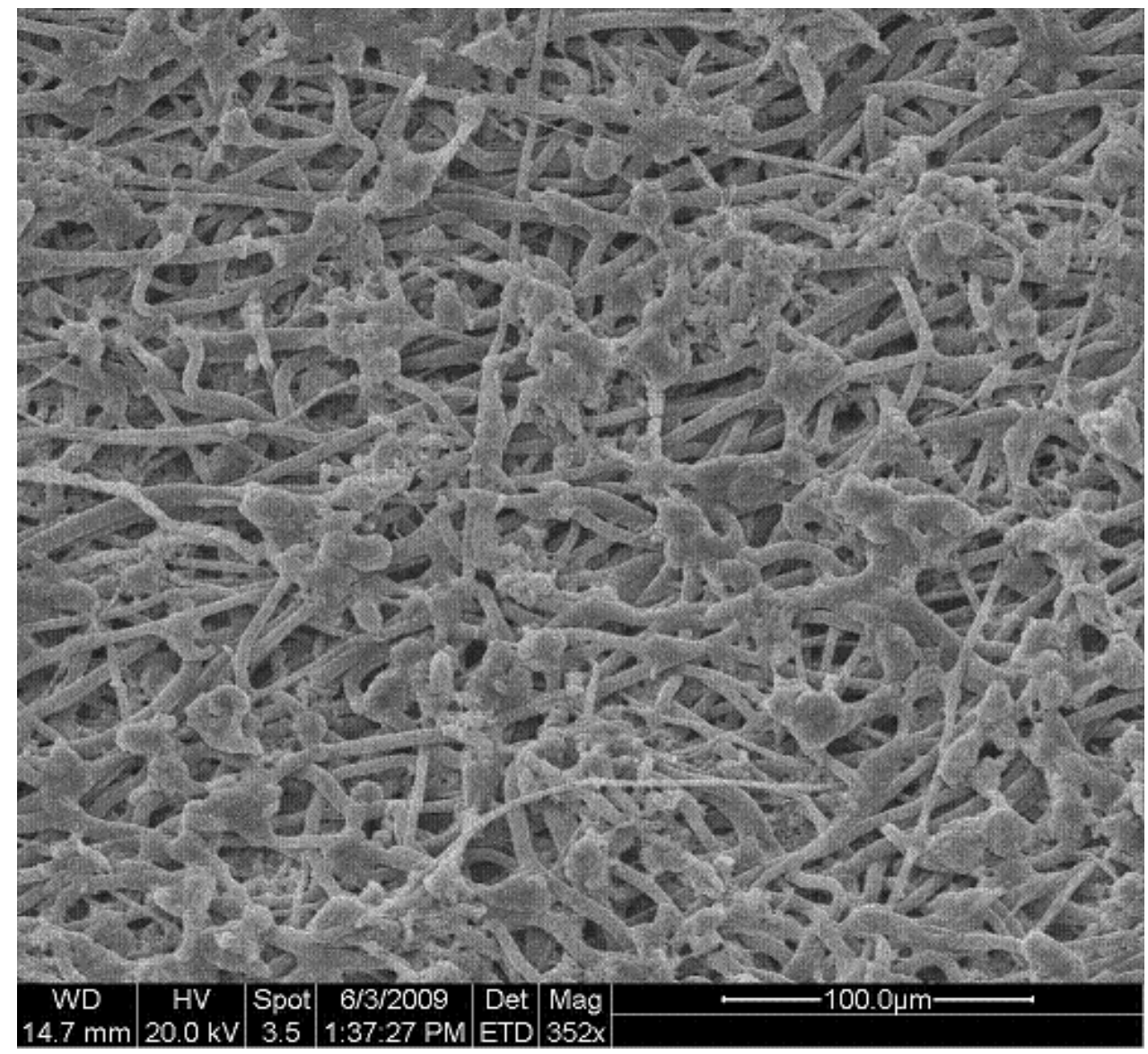

Figure 42 - HUVEC sodded PLGA scaffold after 24 hrs continuous flow culture at 15 RPM. The appearance of some cell spreading is evident.

SEM images of BVM04 (90 RPM, 6 days) showed few areas of good HUVEC coverage on the lumen of the PLGA scaffold (Figure 43). To date, this is the best cell spreading seen for HUVECs on any scaffold material in the Cal Poly Tissue Engineering Lab. 


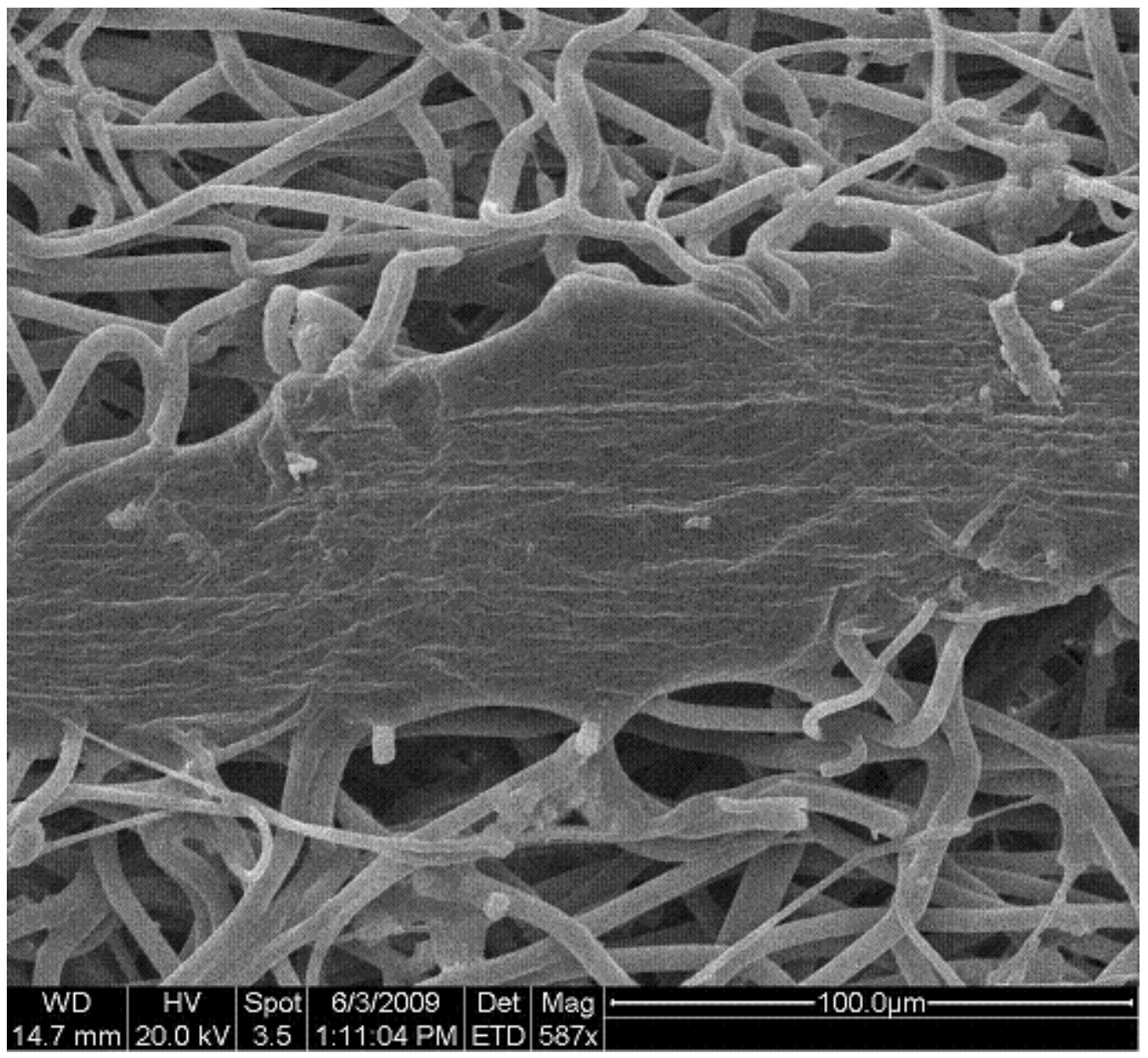

Figure 43 - HUVEC spreading shown on the lumen of the electrospun PLGA scaffold that was cultured for 6 days at 90PRM.

No conclusion could be drawn for SEM analysis of scaffold cross sections for BVM01-

04. However, images are a good example of the highly porous and fibrous architecture developed by electrospinning (Figure 44). In addition, no cells were seen on the lumen of BVM02 and BVM03. 


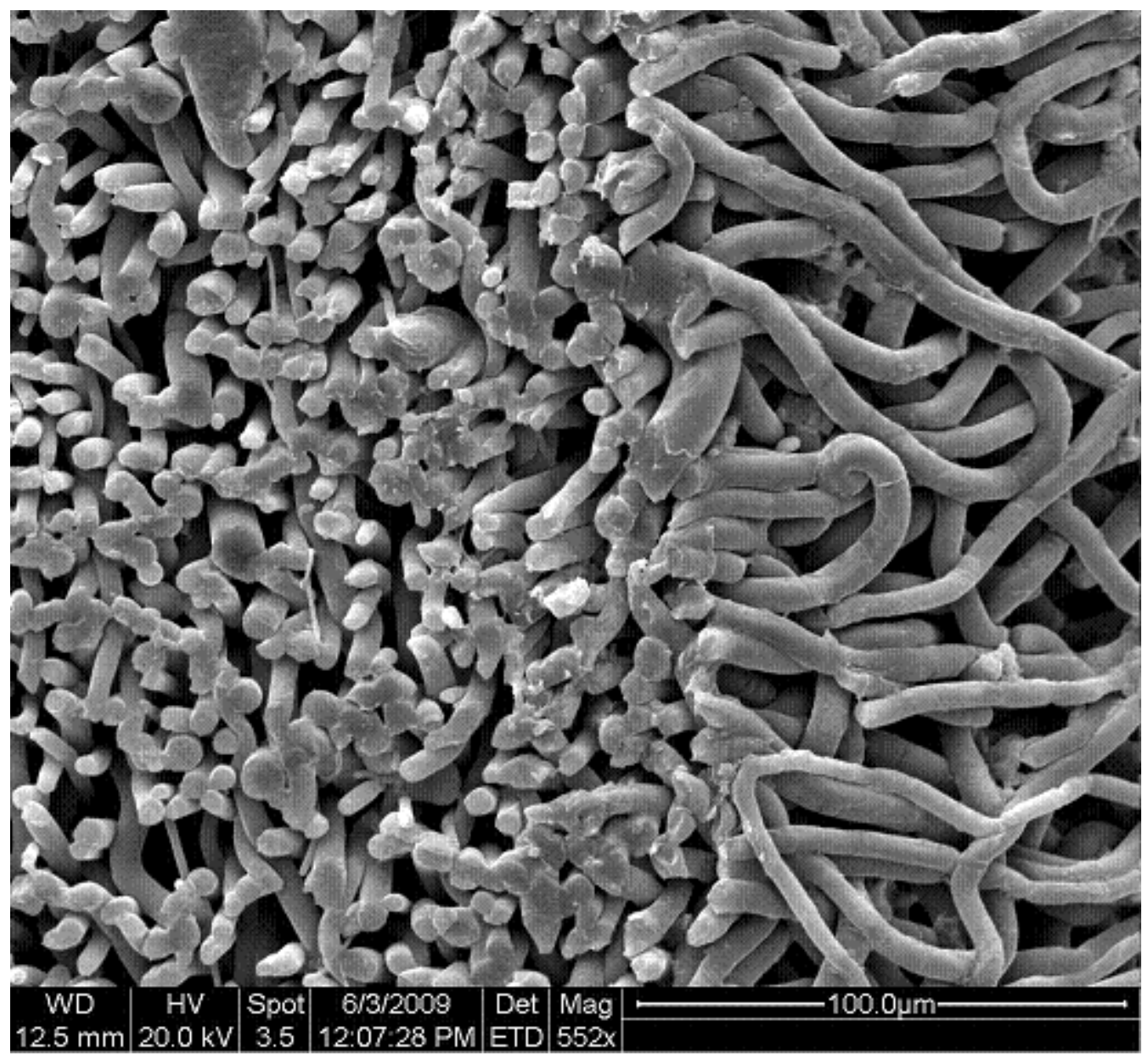

Figure 44 - Cross sectional image of BVM02 showing the fibrous nature of electrospun constructs.

\subsection{Discussion}

\subsubsection{PLGA Response to Media}

PLGA is a biodegradable polymer that degrades by hydrolysis and cleavage of the ester linkages. By-products of PLGA include lactic and glycolic acid. The degradation rate of PLGA is largely dependent upon ratios of PGA in the co-polymer with an increase in degradation corresponding to an increase in PGA. The acidic nature of PLGA degradation by-products was evident in the static media test on $15 \mathrm{wt} \%$ electrospun PLGA samples. In the same study it was 
shown that electrospun PLGA scaffolds subjected to continuously circulating media did not cause an acidic change after 72 hours of flow. Normal body $\mathrm{pH}$ is necessary for cell culture and the stable $\mathrm{pH}$ results of electrospun PLGA under flow supports the use of PLGA for tissue engineering vascular constructs for the BVM.

Electrospun PLGA scaffolds have been used extensively for tissue engineering with a variety of cell cultures in different bioreactor systems. Porous PLGA scaffolds have shown evidence of good cellular attachment and scaffold integrity under continuous flow culture systems [140]. Only one known study suggested electrospun PLGA vascular scaffolds placed under physiologic pressurized flow conditions will collapse as a result of low resistance to shear stress and rapid degradation [141]. Looking into the claim further, it was found that PLGA (50:50; PLA:PGA) was co-spun with collagen when collapse of scaffolds under physiological conditions was observed [142]. Therefore, lack of integrity of the PLGA(50:50)/collagen scaffold could be due to the faster degrading polymer and the addition of biologics in combination with high pressure and flow conditions. Subsequently, integrity of electrospun PLGA (75:25) under low flow, low pressure conditions would be expected for the scaffold developed in this thesis. However, SEM images of $15 \mathrm{wt} \%$ PLGA scaffolds exposed to low flow conditions showed multiple cracked and broken fibers on the vessel lumen. Although flow cannot be ruled out, fiber breakage could be a result of drying of scaffolds in the desiccator when removed from the BVM chamber. Scaffolds were noticeably cracked on the surface during SEM preparation after complete drying. Also, scaffolds could have been mishandled during cutting and sectioning during the SEM preparation. Another cause of cracking could be due to the age of the constructs before the start of the flow condition test. Both SPIN 4 and SPIN 7 were produced several weeks before this test was conducted, with SPIN 4 being the oldest. However, 
there was no difference in appearance between the two constructs in terms of fiber morphology and breakage. Overall fiber surfaces appeared smooth, which could support the hypothesis that drying caused the cracking and breakage of the fibers. Results of tensile testing support stability of scaffolds under such low flow, low shear stress conditions due to good mechanical properties. However, SEM results of HUVEC sodded PLGA scaffolds presented later in this section, show no fiber breakage after 6 days of flow. Further investigations would be necessary to determine the exact cause of fiber breakage. In order to ensure integrity of the PLGA constructs, investigations should determine degradation rates under various durations of flow, and determine an alternative drying method prior to SEM analysis. Also shelf life of the electrospun PLGA should be investigated to ensure constructs are used prior to the onset of degradation. In conclusion, several controlled experiments would be necessary to determine the exact cause of fiber cracking after flow conditioning, as well as to ensure the structural integrity of electrospun PLGA scaffolds is stable during culture in the BVM system.

\subsubsection{Results of Electrospun PLGA Cultured with HUVECs}

BBI staining of HUVEC sodded 15 wt\% electrospun PLGA scaffolds was conducted and results indicate the presence of cells within the pores on the luminal surface of the scaffolds. Results suggest electrospun PLGA scaffolds have potential for vascular tissue engineering as a replacement to ePTFE for the BVM system. From a period of 24 hours to 6 days of culture, the presence of HUVECs on the lumen, in both proximal and distal directions to flow, had similar coverage qualitatively. Results of BBI staining of the HUVEC sodded PLGA scaffolds also suggest that high porosity of electrospun constructs encourages cellular penetration below the luminal surface. More specifically, the pore size allows for such cellular penetration. Deep cellular penetration is ideal for creating a completely or mostly biological tissue construct in 
vitro. In contrast, the goal of the BVM model is to develop an intimal cellular lining of ECs to test the response of device implantation with the majority of the scaffold providing the necessary structural support of the media and adventia of the human blood vessel. Therefore, the BVM design necessitates a scaffold that can adequately support an intimal lining of endothelial cells. To improve upon the current BVM model with HUVEC sodded constructs, electrospun PLGA scaffolds would need to be developed with smaller pore sizes. Pore size is directly related to the fiber diameter for electrospun constructs. In order to decrease the pore size, production of nanofibrous PLGA scaffolds, rather than micron sized, would need to be investigated.

Results of the solution concentration study of this thesis suggest solution viscosity has the ultimate control over fiber size and consistency. At lower solution viscosities, the presence of bead defects were observed which are inappropriate for tissue engineering, as discussed previously in the introduction of this thesis. However, with further investigation of primary literature, lower solution viscosities can achieve continuous non-beaded fiber networks by adjusting the electrospinning gap distance and flow rate and by changing solution conductivity or surface tension [102]. Fiber diameters from $100 \mathrm{~nm}$ to $800 \mathrm{~nm}$ have been achieved for electrospun PLGA constructs when electrospun at a concentration of $1 \mathrm{~g}$ PLGA in $4 \mathrm{ml}$ THF:DMF (50:50); approximately the same ratio used for 15wt\% PLGA in $\mathrm{CHCl}_{3}$ [132]. Further, literature review of electrospun PLGA in solvents combining THF and DMF suggests changing the solvent could lead to nano-fibrous constructs with smaller pore diameters for better endothelialization of PLGA scaffolds for the BVM.

It can be hypothesized that the addition of DMF to THF lowers the volatility of the solvent, which would consequently allow for more fiber thinning during whipping instability prior to collection. For example, a highly volatile solvent such as $\mathrm{CHCl}_{3}$ or $\mathrm{THF}$ alone may 
evaporate almost completely before collection, thus limiting jet thinning during travel through the air. There is also evidence of especially low flow rates leading to smaller fiber diameters in electrospun PLGA constructs, with nano-fibers achieved at flow rates from $0.1 \mathrm{ml} / \mathrm{hr}$ to $1.2 \mathrm{ml} / \mathrm{hr}$ [132]. Such low flow rates were not investigated in this thesis given the time that would be necessary to spin $3 \mathrm{ml}$ of solution in order to produce one tissue engineering scaffold. Although further investigation of lower flow rates adjusted for electrospinning PLGA would be interesting, the time to scaffold formation would be less than ideal and would not address the scalability and efficiency of the BVM model. Gap distance has also been suggested as a possible parameter for controlling fiber diameter, however such investigations are minimal and more evidence is necessary, due to the complexity of varying processing parameters, to confirm the hypothesis. In conclusion, in order to develop smaller pore sizes to limit HUVEC penetration from sub-luminal areas of electrospun PLGA scaffolds, further investigations of electrospinning processing parameters and solvent/polymer combinations should be investigated. However, electrospun PLGA scaffolds with large pore sizes may be advantageous for investigating dual sodding techniques with SMCs and ECs. The large pores may encourage SMC penetration to form a cellular lining followed by an EC lining on the luminal surface of the scaffold. The SMC lining would act as barrier to EC penetration.

Histology is a necessary and effective method of evaluating tissue engineered constructs. Electrospun PLGA scaffolds were processed with traditional histological procedures and H\&E staining techniques in order to evaluate endothelialization of HUVECs on the lumen of the scaffolds and the cellular depth of penetration as a result of porous scaffold morphology. From results of BBI staining, it was hypothesized that porosity of electrospun PLGA constructs was allowing for sub-luminal cellular penetration. Histology would offer a method of confirming 
that hypothesis. However, during traditional histological processing, all PLGA scaffolds were destroyed or severely deformed. This was further evident after H\&E staining and imaging where evidence of deformed or melted polymer was clearly observed. Further investigations of the potential cause lead to an article by Holy and Yakubovich on Processing Cell-Seeded Polyester Scaffolds for Histology [143]. The article specifically investigated alternative methods of cellular imaging for PLGA scaffolds cultured with cells in vitro. According to the article, traditional histological techniques dissolve polyester scaffolds because of the temperatures and organic solvents used during the processing step where scaffolds are initially coated and infiltrated with paraffin wax [143]. Traditional processing, as used in this thesis, uses hot paraffin and a series of chemical purification steps for prolonged duration. A new protocol was determined by Holy and Yakubovich that successfully stains and sections cultured PLGA scaffolds for $H \& E$ staining in a relatively fast and easy manner. The process is carried out by first staining the PLGA samples with H\&E prior to embedding and sectioning. After staining is complete, the samples can be placed in a 10\% poly(vinyl alcohol) [PVA] solution followed by freezing and cryosectioning [143]. After sectioning, PLGA samples can be further stained or mounted in gelatin mounting medium. The use of this protocol was successful for in vitro cell cultured PLGA (75:25) with complete integrity of scaffold morphology achieved [143]. In conclusion, histology was unsuccessful for imaging electrospun HUVEC sodded PLGA scaffolds. The presence of HUVECs was evident from the histology images; however due to severe scaffold deformation it is inclusive as to the exact location of a cellular lining and the depth of cell penetration. All future histology of PLGA should be conducted in a non-traditional manner to avoid the use of harmful solvents and high temperatures. Investigations into altering the histology processing steps or changing the solvents used can be investigated, however a 
cryostat and cryosectioning protocol, similar to that provided by Holy and Yakubovich, would be the optimum choice to pursue for future electrospun PLGA scaffolds.

Further analysis of HUVEC sodded PLGA scaffolds using SEM analysis provided better insight into cell penetration and coverage. Although images were mostly inclusive, the presence of good HUVEC spreading on BVM04 is highly promising for the further optimization of electrospinning PLGA for ePTFE replacement. Unfortunately cross sectional examination of the cultured PLGA scaffolds showed no evidence of where cells may have migrated if indeed subluminal penetration had occurred. Inconsistent cell coverage on the lumen of the PLGA scaffolds again suggests large pore size allows such cellular penetration thus limiting endothelialization on the scaffold lumen. The absence of and inconsistency of cell coverage could also be a result of a poor HUVEC sodding and culturing protocols. At the current time, there is no established protocol for sodding HUVECs on PLGA scaffolds in the Tissue Engineering Lab at Cal Poly. Most of the process followed was experimental or based off of previous work with a different cell type on ePTFE. It may be possible that more cells are needed during sodding to completely cover the PLGA constructs. Also, flow rate may need to be ramped up at slower time points to avoid displacing cells from the lumen of the grafts before successful attachment can take place. In addition, cells may have been lost due to mishandling during BBI staining and SEM preparation.

In conclusion, electrospun PLGA scaffolds showed promising results for a potential replacement to ePTFE for the BVM model. Optimization of the electrospinning protocol and alternative imaging methods should be investigated. 


\section{Chapter 5 - Discussion and Conclusions}

\subsection{Overview}

Electrospinning is a polymer fabrication technique with promising application in the field of tissue engineering. The process offers a relatively simple method of creating polymer scaffolds with micron to nano-sized fiber diameters in a highly porous network similar to the ECM of native tissue. The high surface per unit volume ratio achieved in electrospun constructs is ideal for encouraging cellular attachment, proliferation and migration. The primary aim of this thesis was to develop electrospun scaffolds for tissue engineering an in vitro blood vessel mimic (BVM) for intravascular device evaluation.

Previously, Cardinal et al. demonstrated the feasibility of evaluating the response of an intimal lining of endothelial cells on the lumen of an ePTFE scaffold to stent implantation in vitro [3]. Such a model could allow for fast, reliable and economical testing of new intravascular devices coming to market prior to in vivo evaluation. The original design by Cardinal et al. has several physiological limitations that must be addressed before effective and scalable use of the model can be initiated. The use of ePTFE scaffolding does not meet the ideal criteria of the BVM design due to high cost, dependency on ePTFE suppliers, and the non-native mechanical properties that will have a significant effect on proper device response when compared to device responses in vivo. In order to improve upon the limitations of the current scaffolding material and to increase the scalability and cost effectiveness of the BVM model, the potential for inhouse production of electrospun polymer scaffolds was investigated. 
The first phase of investigating the effective use of electrospinning for tissue engineering vascular scaffolds was to select an appropriate polymer to be studied. Poly(D,L-lactide-coglycolide) [PLGA] is one of the most well studied polymers for tissue engineering and has been effectively electrospun for a variety of tissue applications [131-133]. Due to controlled degradability, good mechanical properties, and evidence of good EC responses when cultured in vitro, PLGA was determined an appropriate scaffolding material for improving upon the current BVM model [127].

In order to investigate the potential for in-house production of electrospun PLGA scaffolds as a replacement to ePTFE, the second phase of this thesis was to develop and optimize a PLGA electrospinning protocol. The first task of protocol optimization was to study different solution concentrations of PLGA in $\mathrm{CHCl}_{3}$ and the resultant effects on electrospun fiber morphologies. Results indicated that increased solution concentration leads to increased fiber diameters and more consistent fiber morphologies. At 15 wt\% PLGA solution concentration, optimum continuous fiber formations were created with fiber diameters of just a few microns. Solution concentrations less than $15 \mathrm{wt} \%$ PLGA led to increasing numbers of bead defects, which are known to hinder cellular attachment and migration [139]. A final protocol was then developed for electrospinning $15 \mathrm{wt} \%$ PLGA by optimizing flow rate and applied voltage. Electrospun constructs developed at different flow rates and electric field strengths were compared and evaluated based upon measured fiber diameter and mechanical properties. The final protocol for electrospinning $15 \mathrm{wt} \%$ PLGA in $\mathrm{CHCl}_{3}$ included a flow rate of $5.5 \mathrm{ml} / \mathrm{hr}$ and an applied voltage of $12,000 \mathrm{~V}$. At this setting, fiber diameters were achieved between 5-6 microns and radial tensile properties were similar to that of the native vessel (4-5 MPa). 
The third phase of this thesis was to develop several PLGA scaffolds, produced at the final electrospinning protocol, for evaluating HUVEC response and the ability to form an intimal cellular lining when cultured in vitro. Electrospun PLGA scaffolds were cultured from 1 to 6 days in a continuous flow bioreactor according to standard BVM practices set by Cardinal et al. The presence of an EC lining was evaluated by BBI staining, histology, and scanning electron microscopy (SEM). Results of BBI imaging showed good HUVEC coverage over the lumen of each scaffold for all culture durations and flow rates. However, scaffolds cultured at 3 and 6 days showed evidence of sub-luminal cellular penetration. In order to confirm the results seen with BBI imaging, histological and SEM analysis was conducted. From histology it was determined that PLGA constructs cannot withstand the aggressive processing used in traditional histological techniques and no further conclusions could be drawn from the processed sections. Alternative methods of processing and H\&E staining were outlined in detail at the end of Chapter 4 of this report. Further investigation of the remaining HUVEC sodded PLGA scaffolds with SEM did not confirm or disprove the deep cellular penetration hypothesis. SEM images did show evidence of good cell attachment after 24 hours of culture at 15 RPM and areas of good HUVEC spreading after 6 days of culture and 90 RPM. Endothelialization is critical to the success of the BVM model, and results of this study suggest electrospun PLGA scaffolds are promising for tissue engineering an in vitro blood vessel mimic with further optimization of the process.

The importance of the scaffold during device evaluation in the BVM is to maintain structural integrity and to support an endothelial cell lining during culture and throughout the course of intravascular device evaluation. An additional criterion for tissue engineering vascular scaffolds is found in the ECM mimicking micro-structural properties including porosity, pore 
size, and general architecture (fibers, voids etc.) [60]. Pore size is critical to cellular migration and in-growth, as well as nutrient and waste transport. Therefore, tailoring pore size could control the depth of cellular penetration in electrospun tissue engineering scaffolds. Control over pore size and porosity is one of the attractive features of electrospinning. Eichhorn et al. developed a model that suggests an increase in fiber diameter is directly proportional to an increase in pore size of electrospun constructs [110]. This model was confirmed by Balguid et al. who systematically evaluated cellular penetration in response to increasing fiber diameters when cultured with cells in vitro [87]. To improve upon the work of this thesis, electrospun PLGA scaffolds with nano sized fiber diameters should be developed to limit cellular penetration in order to support complete cell coverage on the luminal surface of the scaffold.

To further improve upon the work of this thesis, mechanical properties of electrospun PLGA scaffolds should be addressed. Although tensile properties for electrospun PLGA scaffolds were in an ideal range compared to that of the native vessel, Young's modulus was much greater when compared to literature values. This was evident in the great degree of stiffness observed during scaffold setup in the BVM chambers. In addition, longitudinal mechanical properties were on average higher than their corresponding circumferential values. Radial compliance in response to flow and pressure fluctuations is of great importance when biomimicking the native vessel. It can be assumed that cellular response to device implantation will be largely dependent upon the response of the scaffold used in the BVM design. In order to improve upon the current electrospun PLGA scaffolds, further optimization of processing parameters could lead to improved mechanical properties. Studies have shown that fiber alignment can be used to increase radial compliance of electrospun tissue engineered vascular constructs [58]. Fiber alignment is attributed to the translation and rotation of the mandrel 
during the electrospinning process. In addition, fiber orientation could induce proper cellular alignment during culture. One advantage to ePTFE is the nodal-fiber micro-architecture that induces cellular alignment with the direction of physiological flow. The smooth surface associated with EC alignment is ideal for bio-mimicking the native vessel. Therefore, further investigations into the optimization of rotational and translational speeds, and the corresponding effects on mechanical properties and cellular orientation should be conducted.

In conclusion, the work of this thesis provides the basis for future optimization of electrospinning polymer scaffolds for tissue engineering vascular constructs and the optimization of an in vitro blood vessel mimic for intravascular device evaluation.

\subsection{Future Work}

\subsubsection{Refinement of Electrospinning PLGA Protocol}

In order to improve upon the current PLGA electrospinning protocol to produce nano sized fiber diameters and subsequently smaller pore size, different processing parameters and solvent combinations should be investigated. As discussed previously in Chapter 4 of this report, the addition of DMF to $\mathrm{CHCl}_{3}$ or THF, two highly volatile solvents, could increase the potential for producing nano-fibrous construct morphologies. The solvent use may in fact be the greatest contributor to fiber diameter. Other areas not investigated in the work of this thesis are needle size, gap distance, and controlled atmospheric conditions. Of the three parameters not tested, gap distance may be the most beneficial aspect to pursue; although studies suggest only minor control of fiber size is contributed to gap distance [105]. Controlling atmospheric conditions would be costly to implement and nano-fibrous constructs have been produced consistently with 
no such control. Intuitively, needle size would seem to have a limited effect on morphological properties of electrospun scaffolds, none the less there is still potential for optimization.

\subsubsection{Improving the Electrospinning Apparatus}

The electrospinning apparatus used for the investigation laid out in this thesis was only recently developed and has several limitations that should be addressed for future studies using the device. The following section outlines several areas that need improvement and recommendations as to how those areas can be improved.

Containment chamber: The containment chamber is the area in which the electrospinning apparatus, or more specifically the collector, is contained. The containment chamber is a box closed on all sides, made of acrylic. The front wall of the containment chamber is removable for easy access to the collector. However, the current chamber design is unstable and the front wall does not securely stay in place. A new chamber design should be developed. A simple, low cost fix would be to replace the front wall with a light weight acrylic piece, with hinges connecting the front wall to the top of the containment chamber.

Another addition to the containment chamber would be to add a straight edge tool at the base of the chamber. During electrospinning setup, it is difficult to align the mandrel and collector perpendicular to the syringe and syringe pump. An inexpensive, quick fix to this issue would be to laminate a grid and attach it to the bottom acrylic wall of the chamber. The grid will also aid in measuring and adjusting the gap distance prior to each spin.

High Voltage Power Supply: A major disadvantage to the electrospinning apparatus is the high voltage power supply. With the current system, there is no way to measure the exact 
output voltage applied to the spin process and consequently no way to make fine tune adjustments. The high voltage supply should be replaced with a more accurate system and one that will measure exact output voltage.

Rotator Motor Control: Mandrel rotation is necessary to wrap the polymer jet around the mandrel during electrospinning. Orientation of polymer fibers can be controlled through mandrel rotation speed, with more radial oriented fibers achieved at faster rotational RPMs. The rotator motor control box that controls both translation and rotation of the mandrel in the current electrospinning apparatus is a major limiting factor. The current control box does not report rotational or translational speed. Rotational speed would be necessary to investigate variations in fiber alignment, and the effects on scaffold mechanical properties and cellular response when cultured in vitro. A simple method of fixing this issue would be to measure the speed at which the mandrel rotates using a tachometer. In addition a new control box may be necessary if the high speeds necessary for fiber alignment cannot be reached with the current design.

Syringe Pump Stage: The syringe pump holds the syringe in place and regulates flow during electrospinning. The stage on which the syringe pump sits is unstable and difficult to adjust. A more mechanically stable stage would be a user friendly improvement to the electrospinning setup.

\subsubsection{Summary of Continuing Investigations}

The following section is to provide a summary of recommend studies for the future of electrospinning scaffolds for the BVM model. Some of these suggestions were mentioned previously throughout this report. 
Shelf Life: Due to inconclusive results of fiber breakdown of PLGA scaffolds under flow, shelf life investigations should be conducted. Shelf life will be an important aspect for any degradable polymer being investigated for the tissue engineering lab as it will be necessary to ensure scaffold integrity during culture and device evaluation.

Sterilization: Electrospun PLGA scaffolds returned from EtO gas sterilization severely squished and stiffer than their original form. Re-opening the lumen of the scaffolds and inserting them into the BVM chambers was difficult as a result of this change. Investigations into the primary cause of scaffold change will be necessary to ensure scaffold properties were not compromised due to gas sterilization. Additional sterilization techniques possible for electrospun PLGA would be UV light radiation.

PLGA Use for Dual-Sodding: Results of $15 \mathrm{wt} \%$ electrospun PLGA scaffolds from the work of this thesis showed sub-cellular penetration. An interesting study would be to dual sod these scaffolds with SMCs followed by ECs. The larger pore size associated with micron sized fiber diameters could allow for a deeper SMC cellular layer that would act as a barrier for the EC layer to limit penetration. Additionally, the SMC layer would allow for a more physiological representative tissue construct for device evaluation.

Alternative Imaging Methods: Traditional histological processing methods were too aggressive on electrospun PLGA scaffolds and consequently scaffolds were destroyed. The use of xylene and the high temperatures during initial wax infiltration and processing was most likely the primary cause for breakdown of the PLGA. Alteration of processing methods with different solvents and or time points should be investigated. However, the use of a cryostat and 
cyrosectioning may be the simplest and most effective alternative. Refer to Chapter 4 for a more detailed review of cryostat processing.

Introduction of Biologics: PLGA is still a promising alternative to ePTFE with further investigation. Nevertheless, an alternative path to study would be PLGA/collagen composites. PLGA and collagen can be electrospun together and the combination has shown improved mechanical compliance similar to the native vessel [128]. However, PLGA/collagen composites were reported to not withstand the physiological pressures and flow rates in vitro [141]. Lee et al. showed the ability of PCL/collagen to be electrospun together in the same solution and the final constructs were able to promote cell adhesion in a pulsatile perfusion bioreactor for 4 weeks [141]. Still, incorporation of biologics into the electrospun constructs may provide an enhanced physiological representative construct for improved cellular response and intravascular device evaluation.

\subsection{Conclusion}

The development of an in vitro blood vessel mimic for intravascular device implantation and evaluation could have great potential in pre-clinical testing of new vascular devices. Continued optimization of the current BVM needs to be addressed before effective use of the model can be initiated. The introduction of electrospinning for in-house production of tissue engineering scaffolds addressed the scalability, cost effectiveness, and control over BVM design. Electrospun PLGA scaffolds are promising for tissue engineering vascular constructs for the BVM. Future work is necessary to make the electrospinning process more efficient, as well to further optimize processing parameters to achieve nano-fibrous PLGA constructs for development of a confluent endothelial cell lining on the lumen of the scaffold. 


\section{List of References}

1. Waksman, R., Drug-eluting stents from bench to bed. Cardiovascular radiation medicine, 2002. 3(3-4): p. 226-241.

2. Cantor, W., et al., Provisional stenting strategies: systematic overview and implications for clinical decision-making. Journal of the American College of Cardiology, 2000. 36(4): p. 1142-1151.

3. Cardinal, K., et al., Tissue-engineered vascular grafts as in vitro blood vessel mimics for the evaluation of endothelialization of intravascular devices. Tissue Engineering, 2006. 12(12): p. 3431-3438.

4. Lloyd-Jones, D., et al., Heart Disease and Stroke Statistics--2009 Update. A Report From the American Heart Association Statistics Committee and Stroke Statistics Subcommittee. Circulation, 2008.

5. Naghavi, M., et al., From vulnerable plaque to vulnerable patient a call for new definitions and risk assessment strategies: Part I. 2003, Am Heart Assoc. p. 1664-1672.

6. Hoffman, S., et al., A meta-analysis of randomized controlled trials comparing coronary artery bypass graft with percutaneous transluminal coronary angioplasty: one-to eightyear outcomes. 2003, Am Coll Cardio Found. p. 1293-1304.

7. Eagle, K., et al., ACC/AHA guidelines for coronary artery bypass graft surgery: executive summary and recommendations. Indian Journal of Thoracic and Cardiovascular Surgery, 2000. 16(2): p. 110-128.

8. Schumacher, B., et al., Induction of neoangiogenesis in ischemic myocardium by human growth factors first clinical results of a new treatment of coronary heart disease. 1998, Am Heart Assoc. p. 645-650.

9. Moneta, G. and J. Porter, Arterial substitutes in peripheral vascular surgery: a review. J Long Term Eff Med Implants, 1995. 5(1): p. 47-67.

10. Gangemi, J., et al., Does the additive risk of mitral valve repair in patients with ischemic cardiomyopathy prohibit surgical intervention? Annals of Surgery, 2000. 231(5): p. 710. 
11. Konakci, Z., et al., Alpha-Gal on bioprostheses: xenograft immune response in cardiac surgery. European Journal of Clinical Investigation, 2005. 35(1): p. 17.

12. L'Heureux, N., et al., Technology insight: the evolution of tissue-engineered vascular grafts-from research to clinical practice. Nature Clinical Practice Cardiovascular Medicine, 2007. 4(7): p. 389-395.

13. Ballyk, P., et al., Compliance mismatch may promote graft-artery intimal hyperplasia by altering suture-line stresses. Journal of Biomechanics, 1997. 31(3): p. 229-237.

14. Abbott, W., et al., Effect of compliance mismatch on vascular graft patency. J Vasc Surg, 1987. 5(2): p. 376-82.

15. Stewart, S. and D. Lyman, Effects of a vascular graft/natural artery compliance mismatch on pulsatile flow. J Biomech, 1992. 25(3): p. 297-310.

16. Webb, A., et al., In Vitro Characterization of a Compliant Biodegradable Scaffold with a Novel Bioreactor System. Annals of Biomedical Engineering, 2007. 35(8): p. 1357-1367.

17. Pasic, M., Endothelial cell seeding improves patency of synthetic vascular grafts: manual versus automatized method. European Journal of Cardio-Thoracic Surgery, 1996. 10(5): p. 372-379.

18. Randone, B., et al., Dual Role of VEGF in Pretreated Experimental ePTFE Arterial Grafts. Journal of Surgical Research, 2005. 127(2): p. 70-79.

19. Begovac, P., et al., Improvements in GORE-TEX® Vascular Graft Performance by Carmeda ${ }^{\circledR}$ BioActive Surface Heparin Immobilization. European Journal of Vascular \& Endovascular Surgery, 2003. 25(5): p. 432-437.

20. Walpoth, B., et al., Improvement of patency rate in heparin-coated small synthetic vascular grafts. Circulation, 1998. 98(19 Suppl): p. 23.

21. Conklin, B., et al., Basic Fibroblast Growth Factor Coating and Endothelial Cell Seeding of a Decellularized Heparin-coated Vascular Graft. Artificial Organs, 2004. 28(7): p. 668-675.

22. Ratner, B., et al., Biomaterials Science: An Introduction to Materials in Medicine ed 2San Diego. 2004, Academic Press. 
23. Barron, V., et al., Bioreactors for cardiovascular cell and tissue growth: a review. Annals of Biomedical Engineering, 2003. 31(9): p. 1017-1030.

24. Coronary Angioplasty. Heart and Vascular Diseases 2009 [cited 2009 May 27]; Available from: www.nih.gov.

25. Deconinck, E., et al., Pharmaceutical aspects of drug eluting stents. Journal of Pharmaceutical Sciences, 2008. 97(12).

26. Fischman, D., et al., A randomized comparison of coronary-stent placement and balloon angioplasty in the treatment of coronary artery disease. 1994. p. 496-501.

27. Leon, M., et al., A clinical trial comparing three antithrombotic-drug regimens after coronary-artery stenting. 1998. p. 1665-1671.

28. Schwartz, R., N. Chronos, and R. Virmani, Preclinical restenosis models and drugeluting stents still important, still much to learn. Journal of the American College of Cardiology, 2004. 44(7): p. 1373-1385.

29. Schwartz, R., et al., Preclinical evaluation of drug-eluting stents for peripheral applications recommendations from an expert consensus group. 2004, Am Heart Assoc. p. 2498-2505.

30. Grube, E., et al., TAXUS I six-and twelve-month results from a randomized, double-blind trial on a slow-release paclitaxel-eluting stent for de novo coronary lesions. 2003, Am Heart Assoc. p. 38-42.

31. Bello, Y., A. Falabella, and W. Eaglstein, Tissue-Engineered Skin: Current Status in Wound Healing. American journal of clinical dermatology, 2001. 2(5): p. 305.

32. Davis, M. and J. Vacanti, Toward development of an implantable tissue engineered liver. Biomaterials, 1996. 17(3): p. 365-372.

33. Tziampazis, E. and A. Sambanis, Tissue engineering of a bioartificial pancreas:

modeling the cell environment and device function. Biotechnology progress, 1995. 11(2).

34. Choi, R. and J. Vacanti. Preliminary studies of tissue-engineered intestine using isolated epithelial organoid units on tubular synthetic biodegradable scaffolds. 1997: Elsevier. 
35. Zhu, Y., et al., Protein bonding on biodegradable poly (L-lactide-co-caprolactone) membrane for esophageal tissue engineering. Biomaterials, 2006. 27(1): p. 68-78.

36. Sato, M., et al. Artificial esophagus. 1997: TRANS TECH PUBLICATIONS LTD.

37. Fansa, H., et al., Tissue Engineering of Peripheral Nerves: A Comparison of Venous and Acellular Muscle Grafts with Cultured Schwann Cells. Plastic and reconstructive surgery, 2001. 107(2): p. 485.

38. Temenoff, J. and A. Mikos, Review: tissue engineering for regeneration of articular cartilage. Biomaterials, 2000. 21(5): p. 431-440.

39. Ashiku, S., M. Randolph, and C. Vacanti. Tissue engineered cartilage. 1997: TRANS TECH PUBLICATIONS LTD.

40. Hutmacher, D., Scaffolds in tissue engineering bone and cartilage. Biomaterials, 2000. 21(24): p. 2529-2543.

41. Yoshimoto, H., et al., A biodegradable nanofiber scaffold by electrospinning and its potential for bone tissue engineering. Biomaterials, 2003. 24(12): p. 2077-2082.

42. Lin, V., et al., Ligament tissue engineering using synthetic biodegradable fiber scaffolds. Tissue Engineering, 1999. 5(5): p. 443-451.

43. Goh, J., et al., Tissue-engineering approach to the repair and regeneration of tendons and ligaments. Tissue Engineering, 2003. 9(4, Supplement 1): p. 31-44.

44. Yang, S., et al., The design of scaffolds for use in tissue engineering. Part I. Traditional factors. Tissue Engineering, 2001. 7(6): p. 679-689.

45. Mol, A., et al., Tissue engineering of human heart valve leaflets: a novel bioreactor for a strain-based conditioning approach. Annals of Biomedical Engineering, 2005. 33(12): p. 1778-1788.

46. Hoerstrup, S., et al., Tissue engineering of functional trileaflet heart valves from human marrow stromal cells. 2002, Am Heart Assoc. p. 143-150. 
47. Vaz, C., et al., Design of scaffolds for blood vessel tissue engineering using a multilayering electrospinning technique. Acta Biomaterialia, 2005. 1(5): p. 575-582.

48. Ma, Z., et al., Surface engineering of electrospun polyethylene terephthalate (PET) nanofibers towards development of a new material for blood vessel engineering. Biomaterials, 2005. 26(15): p. 2527-2536.

49. Hu, X., et al., Preparation and cell affinity of microtubular orientation-structured PLGA (70/30) blood vessel scaffold. Biomaterials, 2008.

50. Hoerstrup, S., et al., Tissue engineering of small caliber vascular grafts. European Journal of Cardio-Thoracic Surgery, 2001. 20(1): p. 164-169.

51. Ahlswede, K. and S. Williams, Microvascular endothelial cell sodding of 1-mm expanded polytetrafluoroethylene vascular grafts. Arteriosclerosis, Thrombosis, and Vascular Biology, 1994. 14(1): p. 25-31.

52. L'heureux, N., et al., A completely biological tissue-engineered human blood vessel. 1998, FASEB. p. 47-56.

53. Marieb, E. and K. Hoehn, Anatomy \& Physiology. 2008: Pearson/Benjamin Cummings.

54. Isenberg, B., C. Williams, and R. Tranquillo, Small-diameter artificial arteries engineered in vitro. Circulation research, 2006. 98(1): p. 25-35.

55. Mitchell, S. and L. Niklason, Requirements for growing tissue-engineered vascular grafts. Cardiovascular Pathology, 2003. 12(2): p. 59-64.

56. Boland, E., et al., Electrospinning collagen and elastin: preliminary vascular tissue engineering. Front Biosci, 2004. 9: p. 1422.

57. Bonassar, L. and C. Vacanti, Tissue engineering: the first decade and beyond. Journal of Cellular Biochemistry, 1998. 72.

58. Barnes, C., et al., Nanofiber technology: Designing the next generation of tissue engineering scaffolds. Advanced Drug Delivery Reviews, 2007. 59(14): p. 1413-1433. 
59. Blitterswijk, C.V., Tissue Engineering. First ed. Academic Press Series in Biomedical Engineering, ed. P.D. Joseph Bronzino. Vol. 12. 2008, Hartford: Academic Press. 740.

60. Boland, E., P. Espy, and G. Bowlin, Tissue engineering scaffolds. Encyclopedia of Biomaterials and Biomedical Engineering: p. 1-9.

61. Thapa, A., T. Webster, and K. Haberstroh, Polymers with nano-dimensional surface features enhance bladder smooth muscle cell adhesion. Journal of Biomedical Materials Research, 2003. 67(4): p. 1374-1383.

62. Miller, D., K. Haberstroh, and T. Webster, Mechanism (s) of increased vascular cell adhesion on nanostructured poly (lactic-co-glycolic acid) films. Journal of Biomedical Materials Research, 2005. 73(4): p. 476-484.

63. Heydarkhan-Hagvall, S., et al., Three-dimensional electrospun ECM-based hybrid scaffolds for cardiovascular tissue engineering. Biomaterials, 2008.

64. Bhattarai, S., et al., Novel biodegradable electrospun membrane: scaffold for tissue engineering. Biomaterials, 2004. 25(13): p. 2595-2602.

65. Zein, I., et al., Fused deposition modeling of novel scaffold architectures for tissue engineering applications. Biomaterials, 2002. 23(4): p. 1169-1185.

66. Weinberg, C. and E. Bell, A blood vessel model constructed from collagen and cultured vascular cells. Science, 1986. 231(4736): p. 397-400.

67. Niklason, L., et al., Functional arteries grown in vitro. Science, 1999. 284(5413): p. 489.

68. Cheneau, E., et al., Time course of stent endothelialization after intravascular radiation therapy in rabbit iliac arteries. 2003, Am Heart Assoc. p. 2153-2158.

69. Harris, L., B. Kim, and D. Mooney, Open pore biodegradable matrices formed with gas foaming. Journal of Biomedical Materials Research, 1998. 42(3).

70. Nam, Y., J. Yoon, and T. Park, A novel fabrication method of macroporous biodegradable polymer scaffolds using gas foaming salt as a porogen additive. Journal of Biomedical Materials Research, 2000. 53(1). 
71. Liao, C., et al., Fabrication of porous biodegradable polymer scaffolds using a solvent merging/particulate leaching method. Journal of Biomedical Materials Research, 2002. 59(4): p. 676-681.

72. Oh, S., et al., Fabrication and characterization of hydrophilic poly (lactic-co-glycolic acid)/poly (vinyl alcohol) blend cell scaffolds by melt-molding particulate-leaching method. Biomaterials, 2003. 24(22): p. 4011-4021.

73. Mikos, A., et al., Preparation of poly (glycolic acid) bonded fiber structures for cell attachment and transplantation. Journal of Biomedical Materials Research, 1993. 27(2).

74. Wang, F., et al., Precision extruding deposition and characterization of cellular poly-ecaprolactone tissue scaffolds. Rapid Prototyping Journal, 2004. 10(1): p. 42-49.

75. Nam, Y. and T. Park, Porous biodegradable polymeric scaffolds prepared by thermally induced phase separation. Journal of Biomedical Materials Research, 1999. 47(1).

76. Lo, H., M. Ponticiello, and K. Leong, Fabrication of controlled release biodegradable foams by phase separation. Tissue Engineering, 1995. 1(1): p. 15-28.

77. Kang, H., Y. Tabata, and Y. Ikada, Fabrication of porous gelatin scaffolds for tissue engineering. Biomaterials, 1999. 20(14): p. 1339-1344.

78. Sill, T. and H. von Recum, Electrospinning: Applications in drug delivery and tissue engineering. Biomaterials, 2008. 29(13): p. 1989-2006.

79. Doshi, J. and D. Reneker. Electrospinning process and applications of electrospun fibers. 1993.

80. Formhals, A., Method and apparatus for the production of fibers. 1934, Google Patents.

81. Formhals, A., Artificial thread and method of producting same. 1940, Google Patents.

82. Formhals, A., Method and apparatus for the production of artifical fibers. 1939, Google Patents.

83. Taylor, G., Electrically Driven Jets. Proceedings of the Royal Society of London. Series A, Mathematical and Physical Sciences (1934-1990), 1969. 313(1515): p. 453-475. 
84. Baumgarten, P., Electrostatic spinning of acrylic microfibers. Jour. of Colloid and Interface Science. 36(1): p. 9.

85. Deitzel, J., et al., The effect of processing variables on the morphology of electrospun nanofibers and textiles. Polymer, 2001. 42(1): p. 261-272.

86. Huang, C., et al., Electrospun polymer nanofibres with small diameters. Nanotechnology, 2006. 17(6): p. 1558.

87. Balguid, A., et al., Tailoring fiber diameter in electrospun poly (e-caprolactone) scaffolds for optimal cellular infiltration in cardiovascular tissue engineering. Tissue Eng, 2008. 14.

88. Yang, F., et al., Electrospinning of nano/micro scale poly (l-lactic acid) aligned fibers and their potential in neural tissue engineering. Biomaterials, 2005. 26(15): p. 26032610.

89. Matthews, J., et al., Electrospinning of Collagen Nanofibers. BiomacromoleculesWashington-, 2002. 3(2): p. 232-238.

90. McManus, M., et al., Electrospun fibrinogen: feasibility as a tissue engineering scaffold in a rat cell culture model. Journal of Biomedical Materials Research Part A, 2007(2).

91. Reneker, D. and A. Yarin, Electrospinning jets and polymer nanofibers. Polymer, 2008.

92. Buschle-Diller, G., et al., Release of antibiotics from electrospun bicomponent fibers. Cellulose, 2007. 14(6): p. 553-562.

93. Xie, J., X. Li, and Y. Xia, Putting Electrospun Nanofibers to Work for Biomedical Research. Macromolecular Rapid Communications, 2008. 29(22).

94. Yarin, A., S. Koombhongse, and D. Reneker, Bending instability in electrospinning of nanofibers. Journal of Applied Physics, 2001. 89: p. 3018.

95. Pham, Q., U. Sharma, and A. Mikos, Electrospinning of Polymeric Nanofibers for Tissue Engineering Applications: A Review. Tissue Engineering, 2006. 12(5): p. 1197-1211. 
96. Zong, X., et al., Structure and process relationship of electrospun bioabsorbable nanofiber membranes. Polymer, 2002. 43(16): p. 4403-4412.

97. Buchko, C., et al., Processing and microstructural characterization of porous biocompatible protein polymer thin films. Polymer-London-, 1999. 40: p. 7397-7407.

98. Mo, X., et al., Electrospun P (LLA-CL) nanofiber: a biomimetic extracellular matrix for smooth muscle cell and endothelial cell proliferation. Biomaterials, 2004. 25(10): p. 1883-1890.

99. Boland, E., et al., Electrospinning polydioxanone for biomedical applications. Acta Biomaterialia, 2005. 1(1): p. 115-123.

100. Boland, E., et al., Tailoring Tissue Engineering Scaffolds Using Electrostatic Processing Techniques: A Study Of Poly(glycolic acid) Electrospinning. Journal of Macromolecular Science, Part A, 2001. 38(12): p. 1231-1243.

101. Demir, M., et al., Electrospinning of polyurethane fibers. Polymer, 2002. 43(11): p. 3303-3309.

102. Fong, H., I. Chun, and D. Reneker, Beaded nanofibers formed during electrospinning. Polymer-London-, 1999. 40: p. 4585-4592.

103. Lin, T., H. Wang, and X. Wang, The charge effect of cationic surfactants on the elimination of fibre beads in the electrospinning of polystyrene. NANOTECHNOLOGY, 2004. 15(9): p. 1375-1381.

104. Megelski, S., et al., Micro-and nanostructured surface morphology on electrospun polymer fibers. Macromolecules, 2002. 35(22): p. 8456-8466.

105. Jaeger, R., et al. Electrospinning of ultra-thin polymer fibers: Hüthig \& Wepf.

106. Miller, D., et al., Endothelial and vascular smooth muscle cell function on poly (lacticco-glycolic acid) with nano-structured surface features. Biomaterials, 2004. 25(1): p. 5361.

107. Bowlin, G., et al., Electrospinning of polymer scaffolds for tissue engineering. Tissue Engineering and Biodegradable Equivalents: Scientific and Clinical Applications, 2002: p. $165-78$. 
108. Zhang, Y., et al., Electrospinning of gelatin fibers and gelatin/PCL composite fibrous scaffolds. Journal of Biomedical Materials Research, 2005(1).

109. Li, W., et al., Electrospun nanofibrous structure: A novel scaffold for tissue engineering. Journal of Biomedical Materials Research, 2002. 60(4): p. 613-621.

110. Eichhorn, S. and W. Sampson, Statistical geometry of pores and statistics of porous nanofibrous assemblies. Journal of The Royal Society Interface, 2005. 2(4): p. 309-318.

111. Pham, Q., U. Sharma, and A. Mikos, Electrospun Poly ([epsilon]-caprolactone) Microfiber and Multilayer Nanofiber/Microfiber Scaffolds: Characterization of Scaffolds and Measurement of Cellular Infiltration. Biomacromolecules, 2006. 7(10): p. 27962805.

112. Boland, E., et al. Electrospinning of tissue engineering scaffolds. 2001.

113. Choi, J., et al., The influence of electrospun aligned poly (varepsiloncaprolactone)/collagen nanofiber meshes on the formation of self-aligned skeletal muscle myotubes. Biomaterials, 2008.

114. $\mathrm{Xu}, \mathrm{C}$., et al., Aligned biodegradable nanofibrous structure: a potential scaffold for blood vessel engineering. Biomaterials, 2004. 25(5): p. 877-886.

115. Srouji, S., et al., 3-D Nanofibrous electrospun multilayered construct is an alternative ECM mimicking scaffold. Journal of Materials Science: Materials in Medicine, 2008. 19(3): p. 1249-1255.

116. Xue, L. and H. Greisler, Biomaterials in the development and future of vascular grafts. Journal of Vascular Surgery, 2003. 37(2): p. 472-480.

117. Wikol, M., et al., Expanded Polytetrafluoroethylene Membranes and Their Applications. Filtration in the Biopharmaceutical Industry, 1998: p. 71.

118. Pevec, W., et al., Femoropopliteal reconstruction with knitted, nonvelour Dacron versus expanded polytetrafluoroethylene. J Vasc Surg, 1992. 16(1): p. 60-5.

119. Password, F., Long-term results of femorotibial bypass with vein or polytetrafluoroethylene. British Journal of Surgery, 1998. 85(7): p. 934-938. 
120. Tillman, B., et al., The in vivo stability of electrospun polycaprolactone-collagen scaffolds in vascular reconstruction. Biomaterials, 2009. 30(4): p. 583-588.

121. Lee, S., et al., The use of thermal treatments to enhance the mechanical properties of electrospun poly (?-caprolactone) scaffolds. Biomaterials, 2008. 29(10): p. 1422-1430.

122. Inoguchi, H., et al., Mechanical responses of a compliant electrospun poly (L-lactide-coe-caprolactone) small-diameter vascular graft. Biomaterials, 2006. 27(8): p. 1470-1478.

123. Boland, E., et al., Utilizing acid pretreatment and electrospinning to improve biocompatibility of poly (glycolic acid) for tissue engineering. Journal of Biomedical Materials Research, 2004. 71(1): p. 144-152.

124. Williamson, M., R. Black, and C. Kielty, PCL-PU composite vascular scaffold production for vascular tissue engineering: Attachment, proliferation and bioactivity of human vascular endothelial cells. Biomaterials, 2006. 27(19): p. 3608-3616.

125. Pedicini, A. and R. Farris, Mechanical behavior of electrospun polyurethane. Polymer, 2003. 44(22): p. 6857-6862.

126. You, Y., et al., In vitro degradation behavior of electrospun polyglycolide, polylactide, and poly (lactide-co-glycolide). Journal of Applied Polymer Science, 2005. 95(2): p. 193200.

127. Seo, H., et al., Effect of PLGA Nano-Fiber/Film Composite on HUVECs for Vascular Graft Scaffold.

128. In Jeong, S., et al., Tissue-engineered vascular grafts composed of marine collagen and PLGA fibers using pulsatile perfusion bioreactors. Biomaterials, 2007. 28(6): p. 11151122.

129. Stitzel, J., et al., Controlled fabrication of a biological vascular substitute. Biomaterials, 2006. 27(7): p. 1088-1094.

130. Hsu, S., H. Tseng, and M. Wu, Comparative In Vitro Evaluation of Two Different Preparations of Small Diameter Polyurethane Vascular Grafts. Artif Organs, 2000. 24(2): p. 119-128. 
131. Zong, X., et al., Electrospun fine-textured scaffolds for heart tissue constructs. Biomaterials, 2005. 26(26): p. 5330-5338.

132. Zhao, L., et al., Preparation and cytocompatibility of PLGA scaffolds with controllable fiber morphology and diameter using electrospinning method. Journal of biomedical materials research. Part B, Applied biomaterials, 2008.

133. Liao, Y., et al., Preparation, characterization, and encapsulation/release studies of a composite nanofiber mat electrospun from an emulsion containing poly (lactic-coglycolic acid). Polymer, 2008.

134. Blackwood, K., et al., Development of biodegradable electrospun scaffolds for dermal replacement. Biomaterials, 2008. 29(21): p. 3091-3104.

135. Kidoaki, S., I. Kwon, and T. Matsuda, Structural Features and Mechanical Properties of In Situ-Bonded Meshes of Segmented Polyurethane Electrospun from Mixed Solvents. Journal Of Biomedical Materials Research Part B, 2006. 76(1): p. 219.

136. Jeong, S., et al., Mechano-active tissue engineering of vascular smooth muscle using pulsatile perfusion bioreactors and elastic PLCL scaffolds. Biomaterials, 2005. 26(12): p. 1405-1411.

137. Kim, T., D. Lee, and T. Park, Controlled protein release from electrospun biodegradable fiber mesh composed of poly (?-caprolactone) and poly (ethylene oxide). International Journal of Pharmaceutics, 2007. 338(1-2): p. 276-283.

138. Uchida, T., et al., Development of biodegradable scaffolds based on patient-specific arterial configuration. Journal of Biotechnology, 2008. 133(2): p. 213-218.

139. Chen, M., et al., Role of Fiber Diameter in Adhesion and Proliferation of NIH 3 T3 Fibroblast on Electrospun Polycaprolactone Scaffolds. Tissue Engineering, 2007. 13(3): p. 579-587.

140. Kim, S., et al., Survival and function of hepatocytes on a novel three-dimensional synthetic biodegradable polymer scaffold with an intrinsic network of channels. Annals of Surgery, 1998. 228(1): p. 8.

141. Lee, S., et al., Development of a composite vascular scaffolding system that withstands physiological vascular conditions. Biomaterials, 2008. 
142. Lee, S., et al., In vitro evaluation of electrospun nanofiber scaffolds for vascular graft application. Journal of Biomedical Materials Research Part A, 2007(4).

143. Holy, C. and R. Yakubovich, Processing cell-seeded polyester scaffolds for histology. Journal of Biomedical Materials Research, 2000. 50(2). 


\section{Appendix A: List of Abbreviations}

\author{
AHA - American Heart Association \\ BD - Becton Dickenson \\ BVM - Blood vessel mimic \\ CABG - Coronary artery bypass graft \\ CAD - Coronary artery disease \\ $\mathrm{CHD}$ - Coronary heart disease \\ $\mathrm{CHCl}_{3}-$ Chloroform \\ CVD - Cardio vascular disease \\ DMA - N,N-dimethylacetamide \\ DMF - Dimethylformamide \\ DNS - Does not state \\ DTAB - dodecyltrimethylammonium \\ EC - Endothelial cell \\ ECM - Extracellular matrix \\ ePTFE - Expanded poly(tetrafluoroethylene) \\ $\mathrm{EtO}$ - Ethylene oxide (gas) \\ FB - Fibroblasts \\ FBS - Fetal bovine serum \\ HEPES - 4-(2-hydroxyethyl)-1- \\ piperazineethanesulfonic acid \\ HCL - Hydrochloric acid \\ HFP - 111333-hexafluro-2-propanol \\ HUVECS - Human umbilical cord vein \\ endothelial cells \\ IMA - Internal mammary artery \\ IPA - Isopropyl alcohol \\ NA - Not applicable
}

$\mathrm{NIH}$ - National Institute of Health

NZW - New Zealand White

PBS - Phosphate buffered saline

PCI - Percutaneous coronary intervention

PCL - Poly( $\varepsilon$-caprolactone)

PDA - Personal digital assistant

PDO - Poly(dioxanone)

PET - Poly(ethyleneterephthalate)

PGA - Poly(glycolic acid)

PLA - Poly(lactic acid)

PLCL - Poly(L-lactide-co-e-caprolactone)

PLGA - Poly(D,L-lactide-co-glycolide)

P(LLA-CL) - Poly(L-lactide - $c o-\varepsilon-$ caprolactone)

PTFE - Poly(tetrafluoroethylene)

PU - Polyurethane

PVA - Poly(vinyl alcohol)

SMC - Smooth muscle cell

SVG - Saphenous vein graft

TEVG - Tissue engineered vascular graft

TBAC - testrabutylammonium

TFA - Trifluoroacetic acid

THF - Tetrahyrdorfuran

UTS - Ultimate Tensile Strength

UV - Ultraviolet

3D - Three dimensional 


\section{Appendix B: Mixing PLGA Solutions}

\begin{tabular}{|l|c|c|c|}
\hline \multicolumn{1}{|c|}{ Materials/Equipment } & Vendor & Part Number & Quantity \\
\hline $\begin{array}{l}\text { Poly(DL - lactide-co-glycolide) (PLGA) } \\
\text { Lactide:Glycolide (75:25) } \\
\text { Mol wt 66,000 - 107,000 }\end{array}$ & $\begin{array}{c}\text { Sigma Life } \\
\text { Science }\end{array}$ & P1941-5G & 5 grams \\
\hline Chloroform, extra dry, water <50ppm, stabilized & $\begin{array}{c}\text { Fisher } \\
\text { Scientific, Inc }\end{array}$ & 326820010 & 1 Liter \\
\hline 10 ml Syringe, Luer-Lok tip & BD & 309604 & $100 /$ Pack \\
\hline Blunt Fill Needle, 18G 1/1/2 (1.2 mm x 40 mm) & BD & 305180 & $100 /$ Pack \\
\hline Analytical Balance & Acculab & ALC-80.4 & 1 \\
\hline Orbital Shaker & FinePCR & SH130 & 1 \\
\hline Vacuum-Pressure Pipette Aid & $\begin{array}{c}\text { Drummond } \\
\text { Scientific Co. }\end{array}$ & P-80991 & 1 \\
\hline Serological Pipet 5 x 1/10 ml & $\begin{array}{c}\text { VWR } \\
\text { International }\end{array}$ & $53283-706$ & NA \\
\hline Clear Glass Vial 20 ml & $\begin{array}{c}\text { VWR } \\
\text { International }\end{array}$ & $15900-002$ & $72 / \mathrm{CS}$ \\
\hline
\end{tabular}

Procedure:

1. Calculate the amount of PLGA resin necessary for the desired weight percent polymer solution using the following equation. (Density of chloroform is $1.48 \mathrm{~g} / \mathrm{ml}$.)

$$
\begin{gathered}
\text { WPP }=\mathrm{m}_{1} /\left(\mathrm{m}_{1}+\mathrm{m}_{2} \mathrm{~b}\right) \\
W P P=\text { Weight percent polymer solution } \\
\mathrm{m}_{1}=\text { mass of polymer }(\mathrm{g}) \\
\mathrm{m}_{2}=\text { mass of solvent }(\mathrm{ml}) \\
\mathrm{b}=\text { density of solvent }(\mathrm{g} / \mathrm{ml})
\end{gathered}
$$

2. Put on safety gloves. (WARNING: Chloroform can have serious side-effects if it comes in contact with skin, eyes or is inhaled or swallowed. Target organs to be effected are kidneys, heart, central nervous system, liver, eyes, reproductive system and skin. Always open chloroform in a hood and wear protective clothing!!) 
3. Remove PLGA (Figure 45) from the freezer and allow it to reach room temperature (5-10 minutes). Doing so prevents condensation when the polymer is exposed to air.

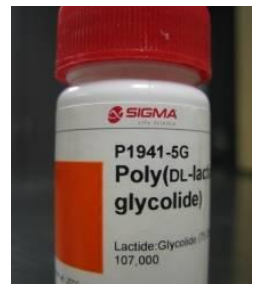

Figure 45 - A $5 g$ bottle of PLGA from Sigma.

4. Weigh out the calculated amount of PLGA using the Acculab Balance (Figure 46) and place the polymer in a $20 \mathrm{ml}$ clear vial. Close the lid immediately.

5. Return unused PLGA to the freezer.

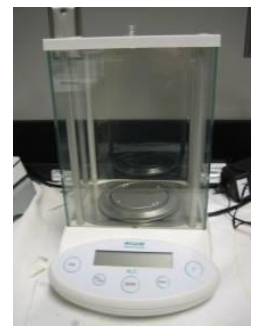

Figure 46 - The Acculab balance.

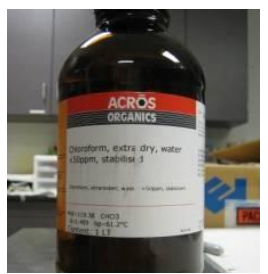

Figure 47 - A 1 liter bottle of chloroform.

7. Gather the Pipette-Aid (Figure 48), a $10 \mathrm{ml}$ disposable pipette and the vial of weighed PLGA and place in the hood with the chloroform.

8. Pipette the desired volume of chloroform into the vial with PLGA.

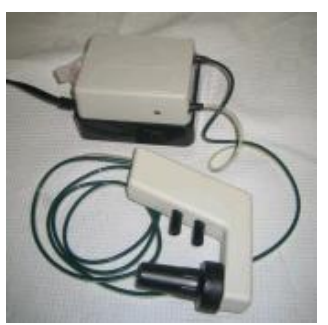

Figure 48 - Pipette aid for obtaining solvent.

Immediately cap the vial as well as the chloroform container to prevent evaporation of chloroform since it is highly volatile.

9. Properly label the solution vial with the WPP, date and your initials (Figure 49).

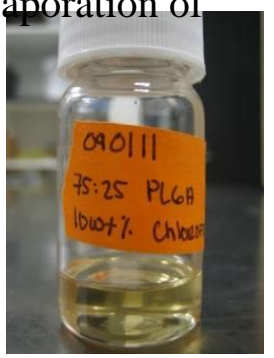

Figure $49-20 \mathrm{ml}$ vial with solution and label. 
10. Wrap vial in aluminum foil to prevent light from entering the solution (chloroform is highly sensitive to light).

11. Place the vial on the orbital shake table (Figure 50). Set the shake table to approximately 3 revolutions per second. Use tape to ensure that the vial will stay upright while on the shake

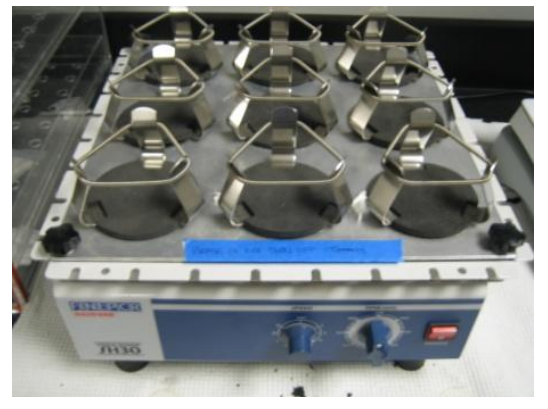

Figure 50 - Orbital shake table for mixing solutions. table. Turn the table on.

12. Allow the solution to mix for 24 hours. After mixing is complete, the solution is usable for up to 48 hours.

13. Remove chloroform container from hood and place back into chemical cabinet.

14. Properly dispose of pipette tip.

15. Clean up work area. 


\section{Appendix C: Electrospinning Protocol}

WARNING: This electrospinning process requires extremely high voltages! Always wear shoes, gloves, and be mindful of what you are touching. Do not attempt to use the electrospinner unless you have been trained by a qualified user.

1. Put on safety gloves and protective clothing. (WARNING: Chloroform can have serious side-effects if it comes in contact with skin, eyes or is inhaled or swallowed. Target organs to be effected are kidneys, heart, central nervous system, liver, eyes, reproductive system and skin. It is possible for residual chloroform to be present on and around the electrospinner during and after a spin!! Make sure to read the MSDS for all chemicals you are working with and know the necessary emergency procedures.)

2. The green ground wire located on the backside of the collector can be removed by pulling it straight out. Unplug the green

ground wire from the collector (Figure 51).

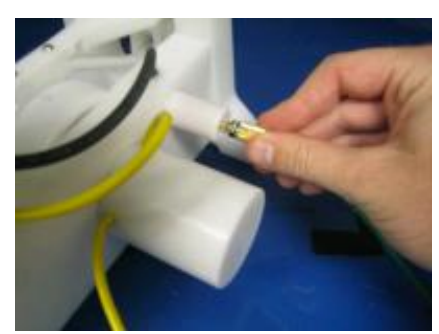

Figure 51 - Green ground wire on back of collector.

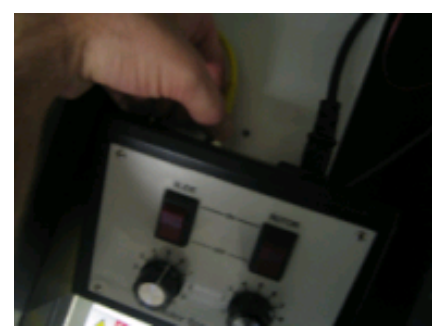

Figure 52 - Yellow power wire connects DC control

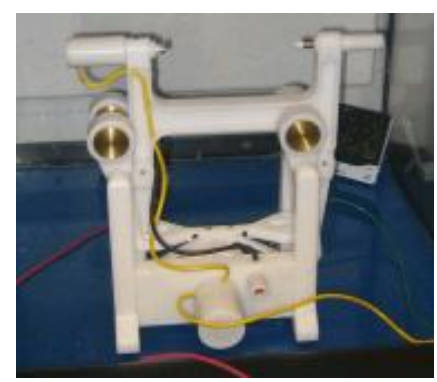

Figure 53 - Entire collector. 
exposed metal, wires and the motor casing. Cleaning before each spin is necessary to remove any residual polymer or dust from the collector that may potentially interfere with the next spin process. Clean the collector using IPA and paper towels. Ensure all residues from both the front and back of the collector including the wires are removed (Figure 54).

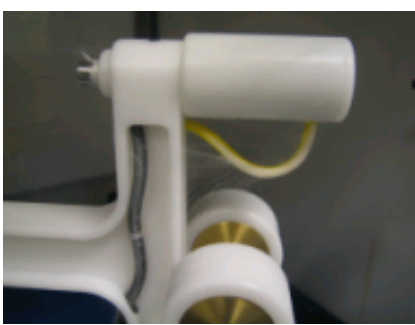

Figure 54 - Polymer residue on wires.

6. During a spin, stray polymer can attach to any wall of the containment chamber and even form webs of polymer between walls. If necessary, clean the inside of the containment chamber with IPA.

7. Replace the collector back inside the containment chamber and reconnect the green ground and yellow power wires.

8. Prepare the mandrel for spinning. Clean the mandrel with IPA to remove any dust, residual polymer from a previous spin, or metal particles. If the mandrel surface is scratched, fine grit sand paper can be used to re-smooth the surface of the mandrel. If sanding is necessary, clean the mandrel with IPA when finished.

9. Attach the mandrel to the collector. When inserting the mandrel, rotate the turn knob until most of the metal chuck is covered, you will feel some resistance. If you go too far the turn knob will spring back.

10. There are three power cords to the right of the fume hood; one for the external power supply, one for the main power and one for the DC motor control box. Plug in all the equipment. 
11. Using a multimeter, check the resistance between the ground connection and the mandrel. Verify that there is some conductivity. Record your measurements (Figure 55).

12. In the fume hood, prepare a $10 \mathrm{ml}$ syringe with an $18 \mathrm{GA}$ fill needle.

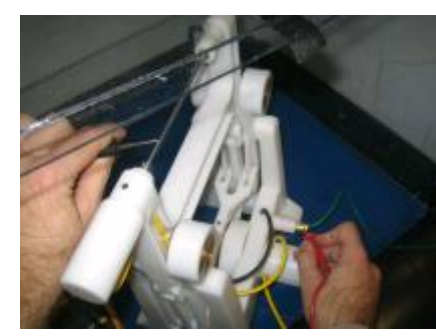

Figure 55 - Checking resistance between mandrel and ground wire.

13. Remove aluminum foil from the PLGA solution vial for better visibility when working with the solution.

14. Solutions may be highly viscous and filling the syringe may take time and require some strength. Make sure to not release pressure on the plunger when drawing solution into the syringe. Acquire just

over $3 \mathrm{ml}$ of the polymer solution into the prepared $10 \mathrm{ml}$ syringe

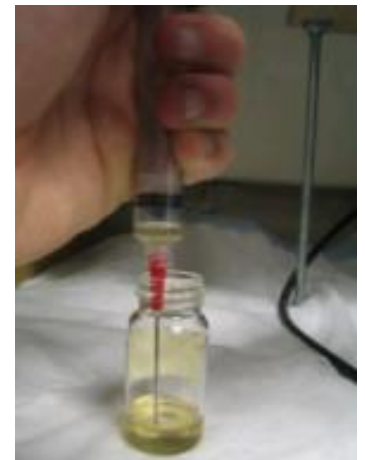

Figure 56 - Acquiring solution into syringe. (Figure 56).

15. Once the solution has been acquired in the syringe, replace the fill needle with an $18 \mathrm{GA}$ Blunt needle.

16. Push the plunger back into the syringe until most of the air is removed and the solution is just in the needle. WARNING: If you push too hard too fast the polymer melt may squirt out. If this happens you will need to attach a new needle.

17. Place the filled $10 \mathrm{ml}$ syringe into the syringe pump. The needle should go through the needle tip hole in the containment chamber wall (Figure 57).

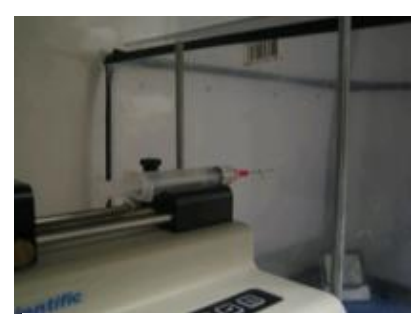

Figure 57 -Positioned syringe in the pump. 
18. Re-position the collector in the containment chamber so that the mandrel and the needle tip are 10 inches apart and perpendicular to each other (Figure 58). NOTE: The side of the collector with green ground wire connection should face away from the needle.

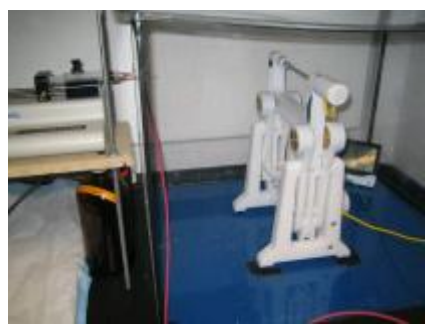

Figure 58 - Proper positioning of the collector.

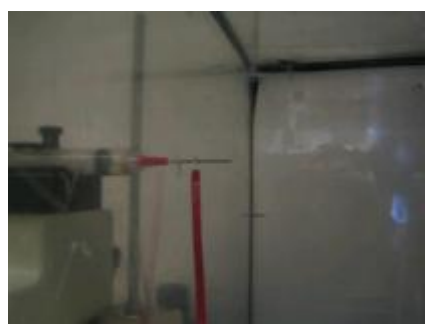

Figure 59 - Red wire hung on needle. needle during the spin, the external $10 \mathrm{~V}$ power source used to regulate high voltage output will burn out. Be sure to hang the wire on the needle securely!

20. The power switch for the syringe pump is located on the back of the pump. Turn the syringe pump on.

21. Enter the desired flow rate and solution volume. The solution volume will determine when the pump will stop. Make sure the screen remains on volume. NOTE: Syringe pump instructions are located in a cabinet close to the fume hood if you need further instructions.

22. Turn on the "Rotate" and "Slide" functions of the collector at the DC motor control box. Ensure the collector is now oscillating back and forth and the mandrel is rotating. If the mandrel is not rotating, you can tap it gently to get it started (Figure 60).

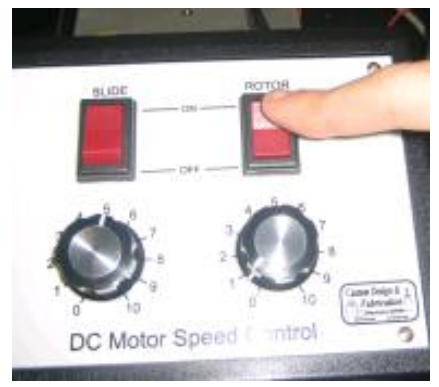

Figure 60 - DC motor switches. 
23. Place the front wall onto the containment chamber. The bottom right corner of the front wall is cut for wires to run through. Secure the wall in place with tape so it does not come off (Figure 61).

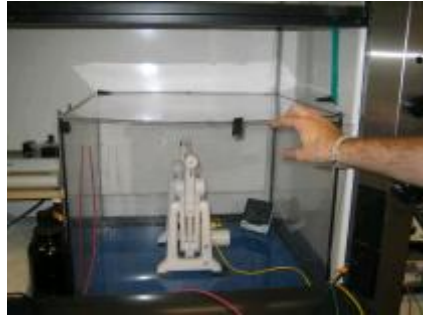

Figure 61 - Front wall placed on chamber.

24. Turn on the external power source and set it to the desired voltage. Turn the external power source off (Figure 62).

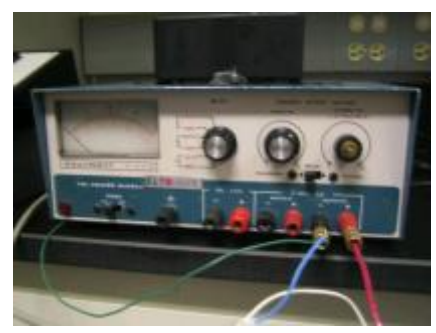

Figure 62 - The external power supply.

Read Steps 25-40 BEFORE beginning the electrospinning process. The following steps for turning $\mathrm{ON}$ and $\mathrm{OFF}$ the electrospinning system must be followed in the exact order listed.

25. Press the "Run" button on the syringe pump. The volume count will begin on the screen and an arrow will flash meaning the solution is now being pushed through the needle.

26. When a droplet forms on the tip of the needle, the process is ready to begin.

27. Turn on the "Main Power" (left switch). Power is on if the light on the AC/DC power converter turns green (Figure 63).

28. Turn on the external source.

29. Prepare to turn on the High Voltage (right switch) [Figure 64]. Look at the droplet of polymer on the end of the syringe and turn High Voltage on. The droplet should disappear.

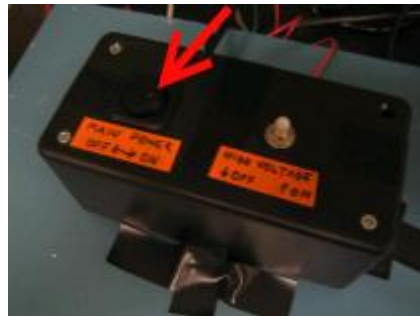

Figure 63 - The Main Power switch.

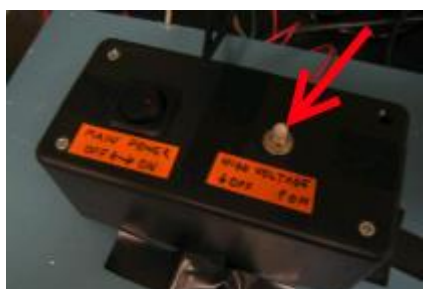

Figure 64 - High Voltage switch. 
30. Monitor the process for the entire spin. The mandrel should slowly start to become coated with the polymer.

31. When all the polymer have been spun, the process should be shut down in the following order.

a. Press the RUN/STOP button on the syringe pump.

b. Turn the HV switch to OFF

c. Turn the Main Power OFF

d. Turn the ROTATE and SLIDE functions on the DC Motor Control box OFF.

32. In order to allow the solvent to fully evaporate, wait a few minutes before removing the mandrel from the containment chamber.

33. Remove the front containment chamber wall.

34. Remove the mandrel with PLGA scaffold.

35. Touch the red high voltage wire to the green ground wire to remove any residual charge.

36. Remove syringe from syringe pump and dispose in sharps container.

37. Unplug all equipment.

38. Properly dispose of all waste and clean up your work station.

39. Transfer the mandrel with the PLGA scaffold to the desiccators for further drying of the scaffold. Allow the scaffold to remain on the desiccators for 24 hours.

40. Remove the scaffold from the mandrel using gauze and carefully twist the scaffold off. 


\section{Appendix D: 303 SS Mandrels}

\begin{tabular}{|c|c|c|c|}
\hline Materials & Vendor & Part Number & Quantity \\
\hline $\begin{array}{c}\text { 303 Stainless Steel, Unpolished } \\
\text { (diameter }=4 \mathrm{~mm}, \text { length }=1 \mathrm{~m}) \\
\text { *Precision Ground not required }\end{array}$ & McMaster Carr & $1274 \mathrm{~T} 13$ & 1 \\
\hline $\begin{array}{c}\text { Small Diameter Drill Bit } \\
\left(1 / 16 \text { " diameter, } 1 / 8^{\prime \prime} \text { shank }\right)\end{array}$ & McMaster Carr & 2841A94 & 1 \\
\hline $\begin{array}{c}\text { Stainless Steel Slotted Spring Pins } \\
(1 / 16 \text { "diameter, } 1 / 2 \text { "length) }\end{array}$ & McMaster Carr & 92383A106 & $100 / \mathrm{Pkg}$ \\
\hline
\end{tabular}

**WARNING: Do not attempt to use the lathe if you have not been properly trained in all operating procedures as well as safety protocols. The lathe can cause severe injury if used improperly!

1. Read and understand all instructions before beginning fabrication.

2. If you have any questions regarding this SOP or machinery operations, contact Dr. Kristen Cardinal for assistance.

3. Cut the 1 meter stainless steel rod into $15 \mathrm{~cm}$ sections using horizontal and/or vertical band saws. There will be six $15 \mathrm{~cm}$ sections and a small scrap portion of steel left at the end. (Note: the final length of each mandrel will be $14 \mathrm{~cm}$ ).

4. Using a lathe, position the bulk of the $15 \mathrm{~cm}$ rod in the chuck, leaving only a small amount of material exposed. Face one end of each $15 \mathrm{~cm}$ rod using a carbide-tipped lathe tool. Set the speed to 1,170 RPM. Chamfer the faced edges using a fine file; there is no need to adjust the speed for this.

5. Prior to drilling the $1 / 16$ " hole, the surface of the rod must be prepared. To prevent bitwandering and provide a better drill bit-surface contact, a file should be used to slightly grind a flat surface on the rod before drilling.

6. Before drilling the hole, provide a few drops of cutting fluid to the bit to help with lubrication. (Note: The key to drilling this size hole is to move slowly and peck the material frequently, however be careful as to not work harden the steel). Using a CNC mill, drill a 1/16" hole through diameter of each $15 \mathrm{~cm}$ rod, approximately $3 \mathrm{~mm}$ from the finished/chamfered end to the center of the hole. Set drill speed to 2,300 RPM and use the 1/16" small diameter drill bit (1/8" shank).

7. Using the lathe, face the unfinished end of each $15 \mathrm{~cm}$ rod. Set speed to 1,170 RPM.

8. Using a \#2 drill bit on the lathe, drill a shallow hole into the faced/un-chamfered end of each $15 \mathrm{~cm}$ rod. Drill just deep enough to attach a live center. Do this for all $15 \mathrm{~cm}$ sections of steel. Set speed to 1,170 RPM. 
9. Secure the $15 \mathrm{~cm}$ rod between the live center and the chuck of the lathe. Expose as much of the rod as is possible. Set speed to 510 RPM. Wet-polish the rod using 600 grit sandpaper and methanol or water. (Note: If dirty or greasy, clean rods before polishing to remove particles and to achieve a better surface finish).

10. Cut the $15 \mathrm{~cm}$ rods down to $14 \mathrm{~cm}$ ensuring to remove the face with the \#2 drill bit hole. Use a band saw to remove the bulk of the material, leaving just enough material to finish the end.

11. Using the lathe, face and chamfer the unfinished end following the same procedure as in step 2.

12. Insert a $1 / 16$ " slotted spring pin into the $1 / 16$ " hole that was drilled in step 3 . This can be best achieved by crimping one end of the pin with pliers to get the pin partly inserted. Then using the pliers, force the pin into the center of the hole.

13. Clean mandrels thoroughly with IPA before use to ensure all oil, dirt and metal particles are removed.

14. Evaluate each mandrel manufactured by ensuring the mandrel properly fits and rotates in the electrospinner. Also, perform a test spin on each mandrel and check for any differences between polymer collection and scaffold removal compared to that of the original mandrels. The new mandrel should be nearly identical to the original. If scaffolds are difficult to remove from the mandrels, further wet polishing on the lathe may be necessary (See Step 6). 


\section{Appendix E: ImageJ Protocol}

1. Open program ImageJ. The following screen will appear.

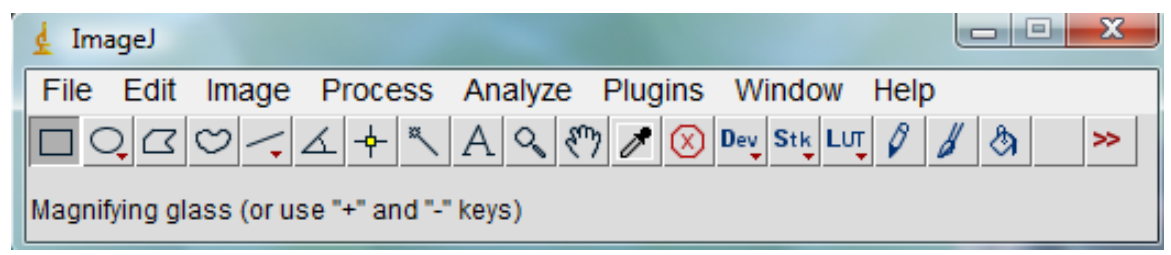

3. Select File > Open. Open a saved SEM image. The image will appear in a separate screen.

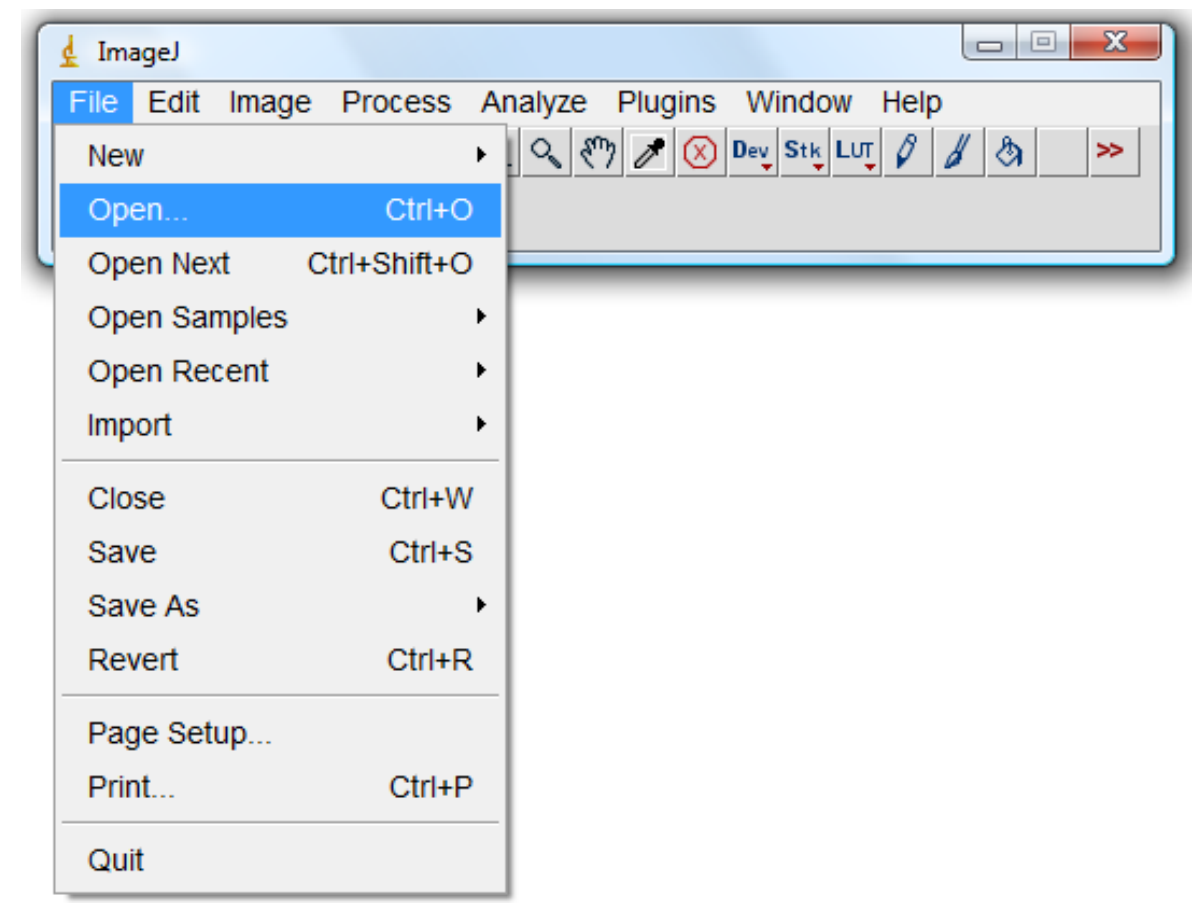

4. Select $\subsetneq$ and draw a line along the SEM image's scale bar. Visually ensure the line is as close to the length of the scale bar as possible as this will affect the outcome of fiber diameter measurements (See image under Step 5 for further clarification). 
5. On the tool bar, select Analyze > Set Scale.

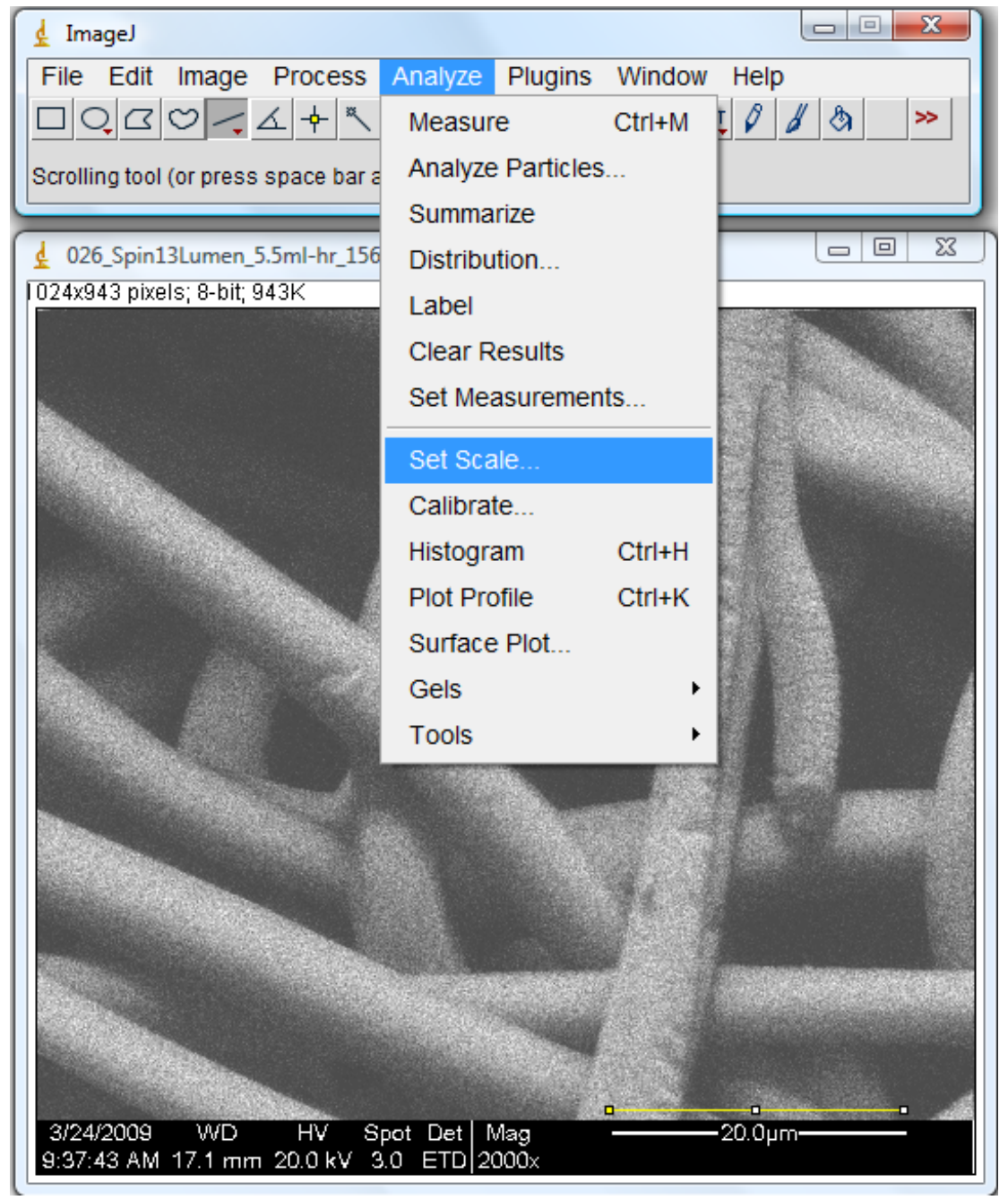

5. Insert known image scale bar distance (ex. $20 \mu \mathrm{m})$. Set pixel aspect ratio to 1.0 and set appropriate unit length (ex. $\mu \mathrm{m})$. Selcect OK. ImageJ is now calibrated to the image.

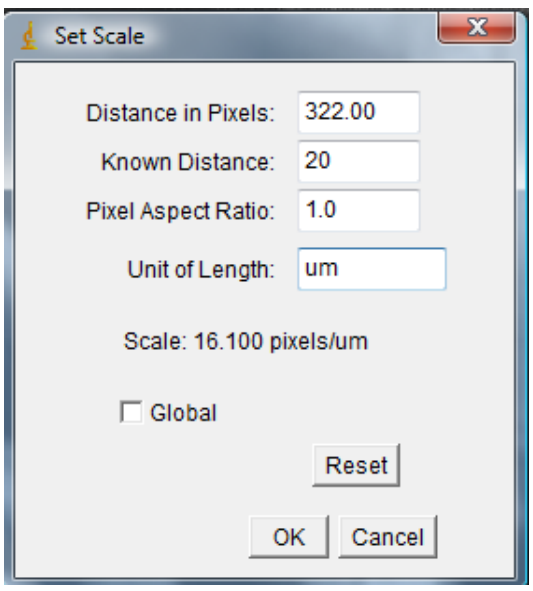


6. From the tool bar, select $\subsetneq$. Draw a line across the diameter of a single fiber to be measured.

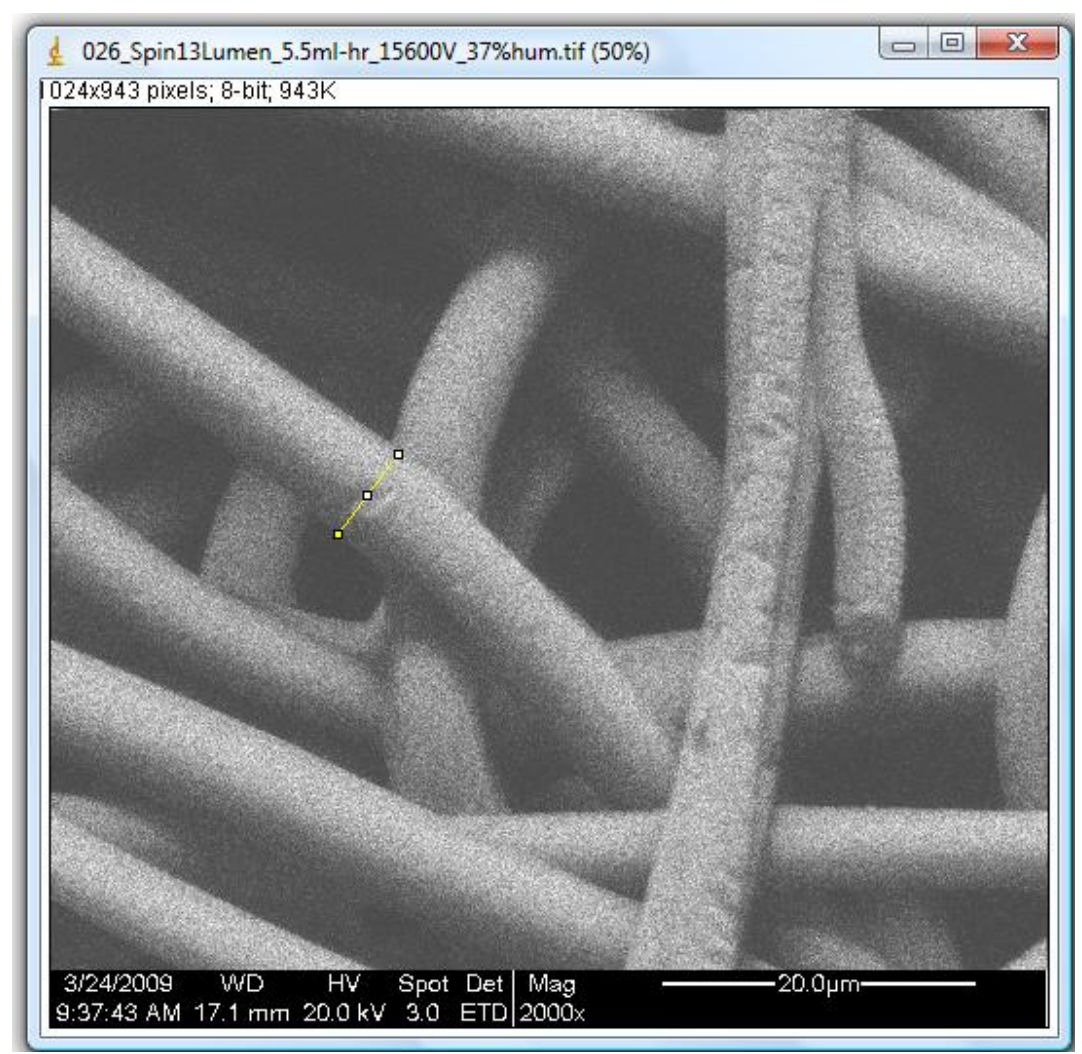

7. From the toolbar select Analyze $>$ Measure. ImageJ will open a new window reporting Results. Fiber diameter is reported as Length in the Results window in proper units (ex. $\mu \mathrm{m})$.

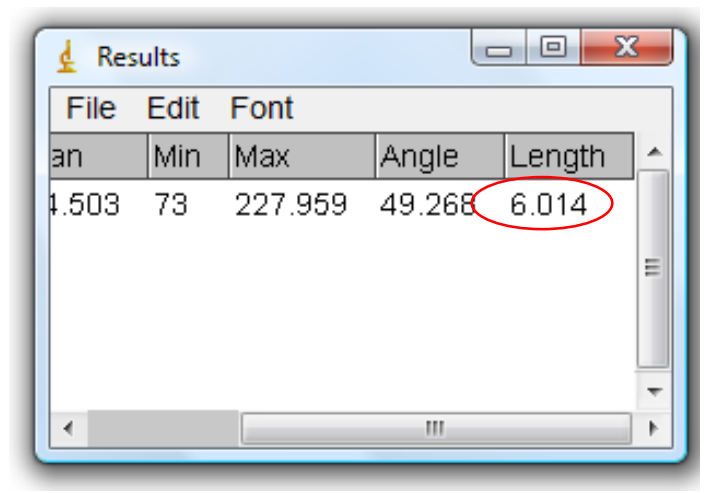

8. Leave the Measure window open and repeat Step 6 for all fiber measurments for that image.

9. Repeat for all images. NOTE: Calibration is only necessary for the first image ONLY if all images being analyzed are at the SAME magnification. Re-calibration will be necessary for images taken at different magnifications. 


\section{Appendix F: Fiber Diameter Measurements}

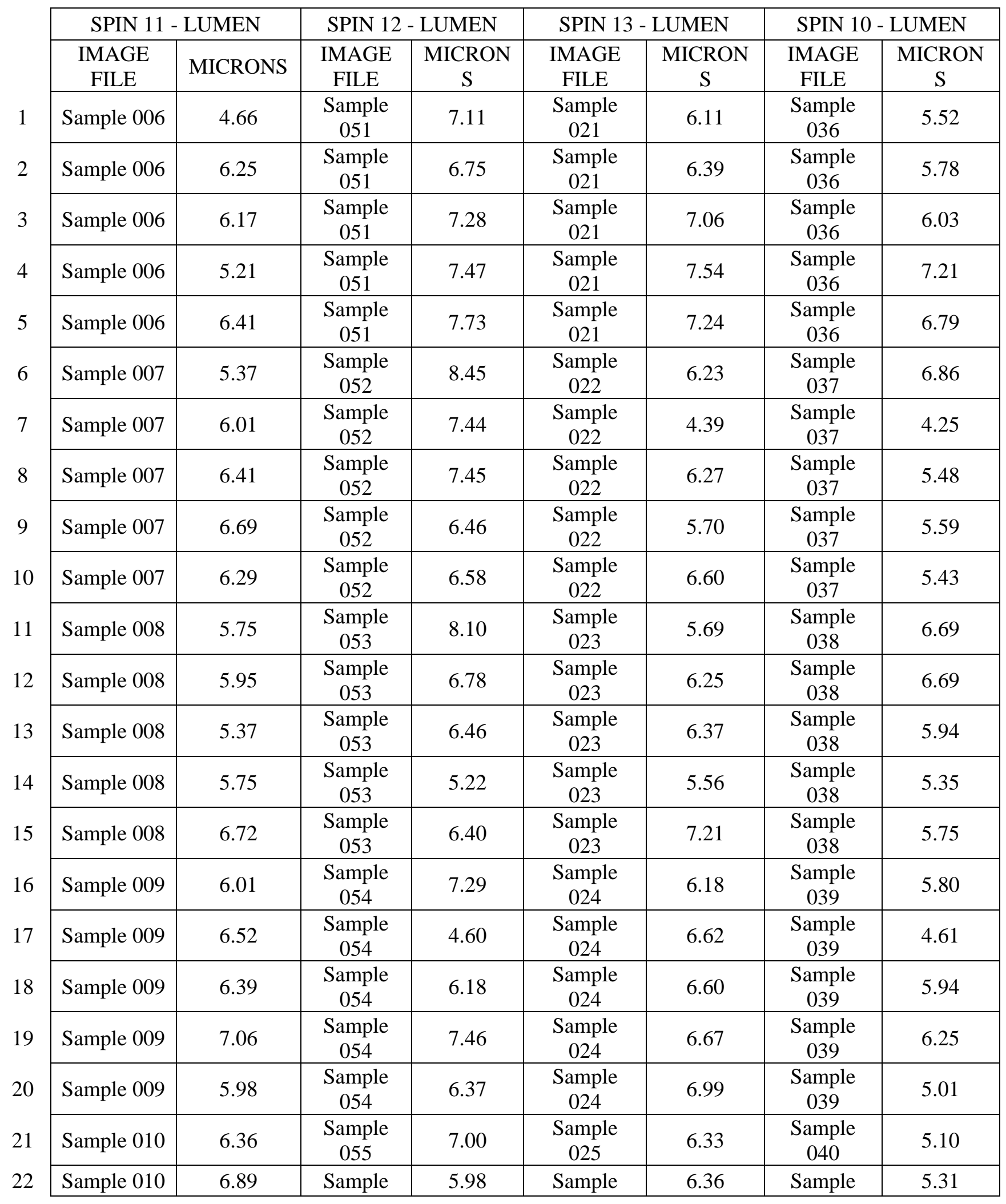




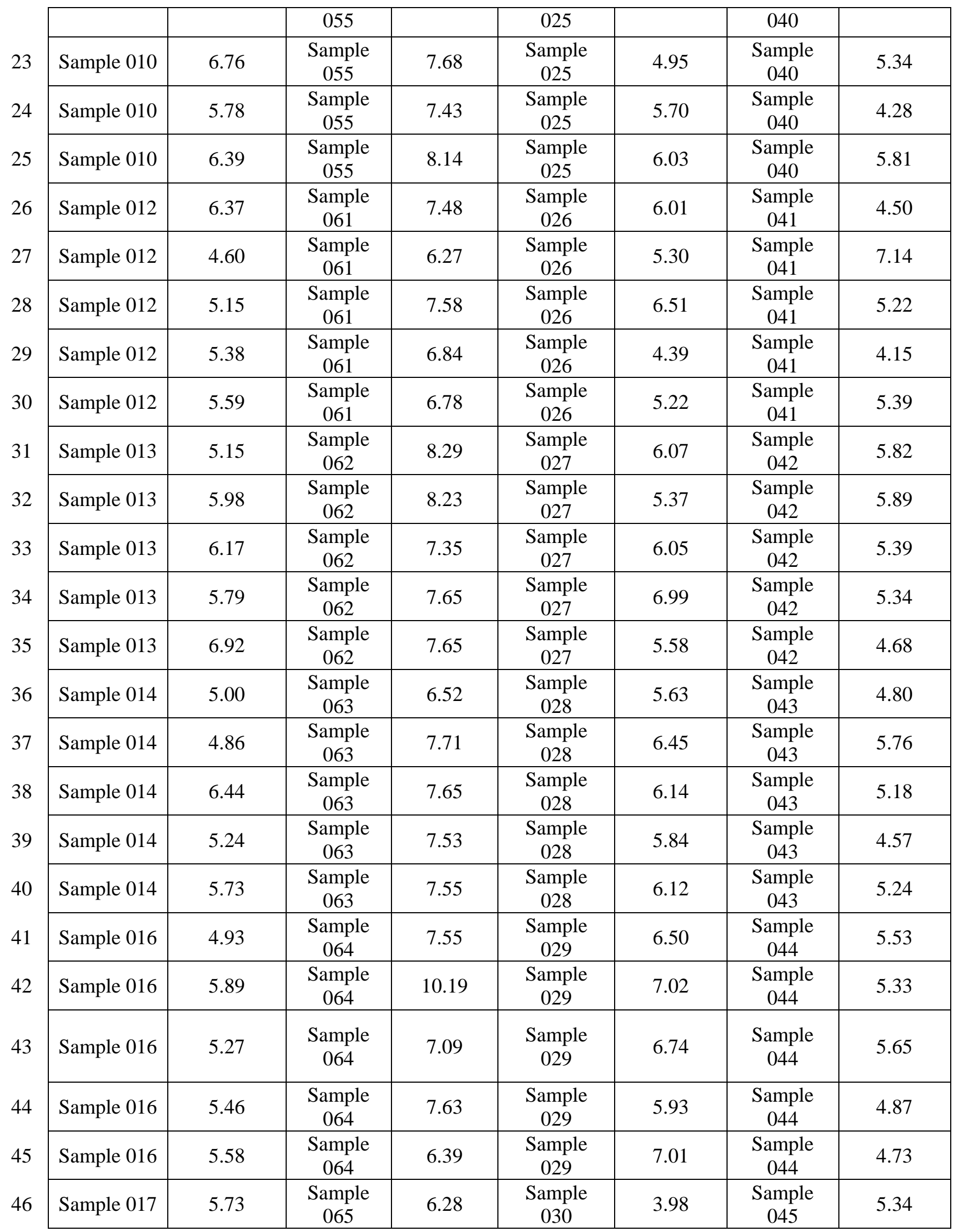




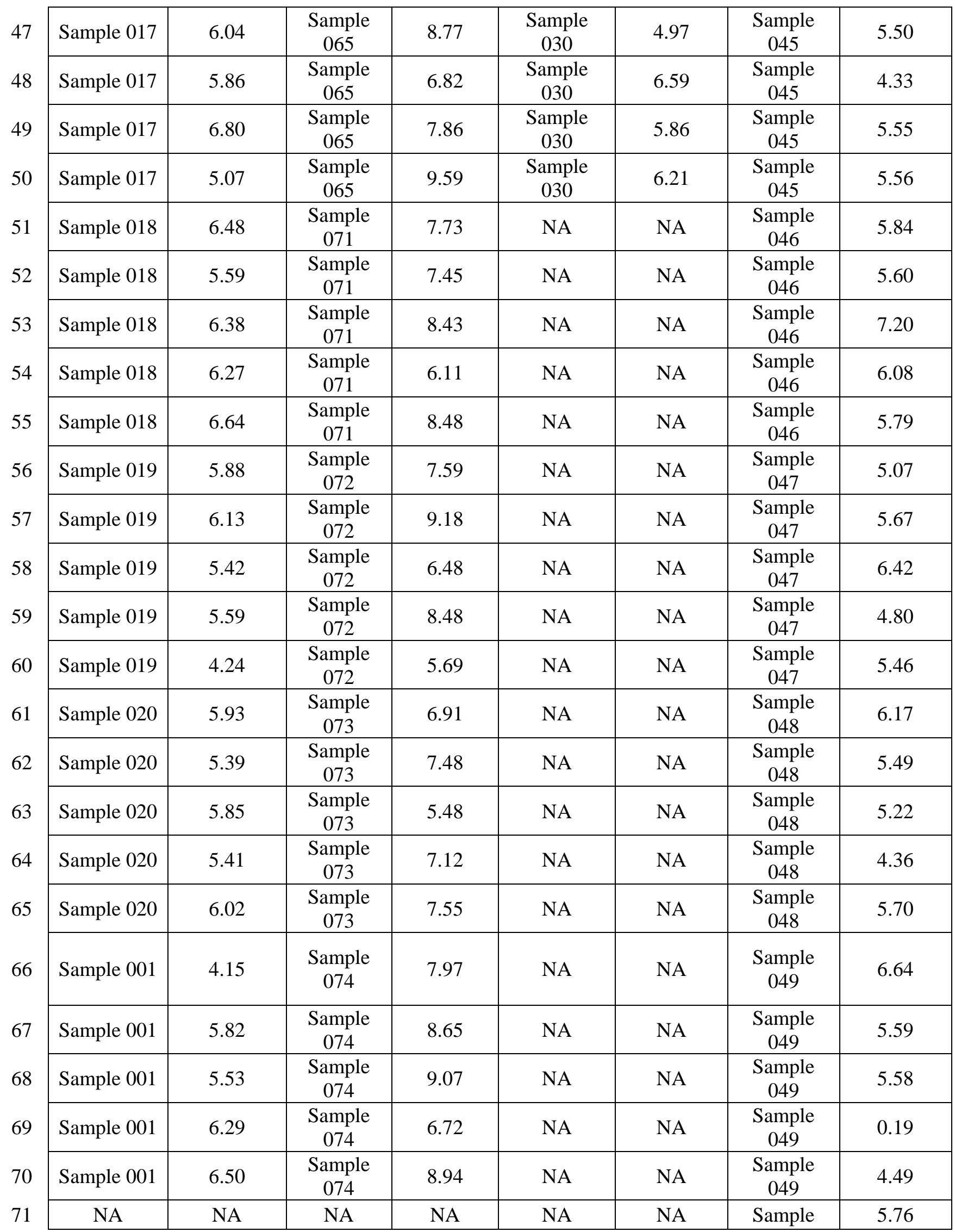




\begin{tabular}{|c|c|c|c|c|c|c|c|}
\hline & & & & & & 050 & \\
\hline NA & NA & NA & NA & NA & NA & $\begin{array}{c}\text { Sample } \\
050\end{array}$ & 5.86 \\
\hline NA & NA & NA & NA & NA & NA & $\begin{array}{c}\text { Sample } \\
050\end{array}$ & 5.99 \\
\hline NA & NA & NA & NA & NA & NA & $\begin{array}{c}\text { Sample } \\
050\end{array}$ & 6.19 \\
\hline NA & NA & NA & NA & NA & NA & $\begin{array}{c}\text { Sample } \\
050\end{array}$ & 5.98 \\
\hline MEAN & 5.85 & MEAN & 7.35 & MEAN & 6.11 & MEAN & 5.49 \\
\hline STDEVE & 0.64 & STDEVE & 1.00 & STDEVE & 0.75 & STDEVE & 0.93 \\
\hline MIN & 4.15 & MIN & 4.60 & MIN & 3.98 & MIN & 0.19 \\
\hline MAX & 7.06 & MAX & 10.19 & MAX & 7.54 & MAX & 7.21 \\
\hline MODE & 6.41 & MODE & 7.45 & MODE & 4.39 & MODE & 5.34 \\
\hline
\end{tabular}




\section{Appendix G: Results of Tensile Testing}

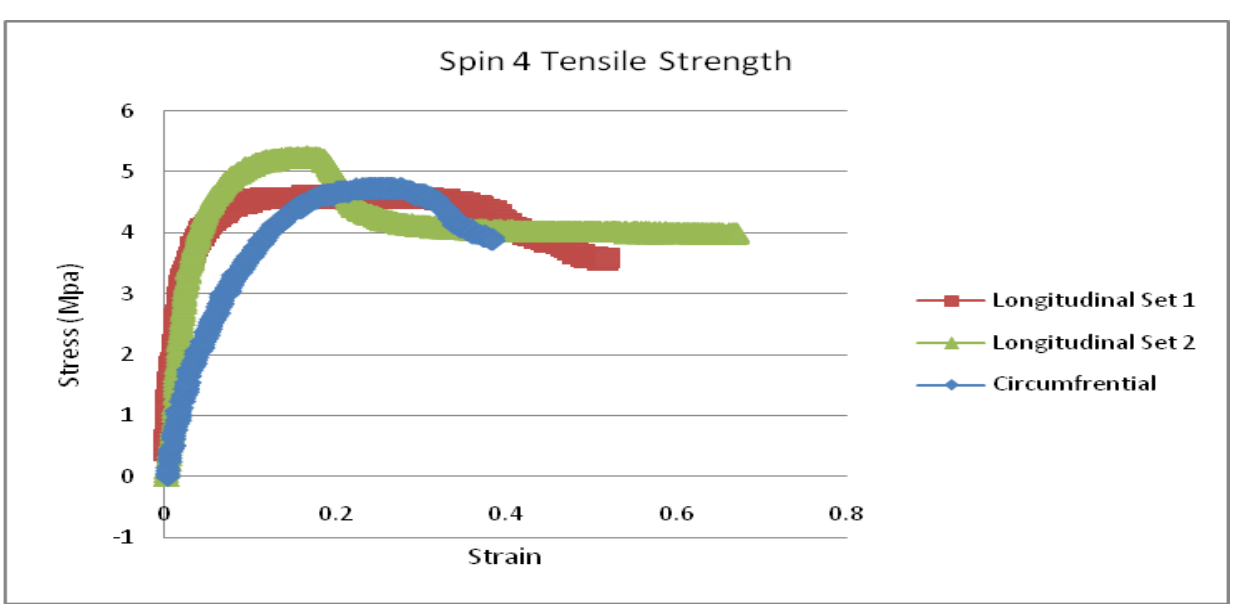

Figure 65 - Tensile strength for SPIN 4, 15 wt\% PLGA in $\mathrm{CHCl}_{3}(5.5 \mathrm{ml} / \mathrm{hr}$; unknown).

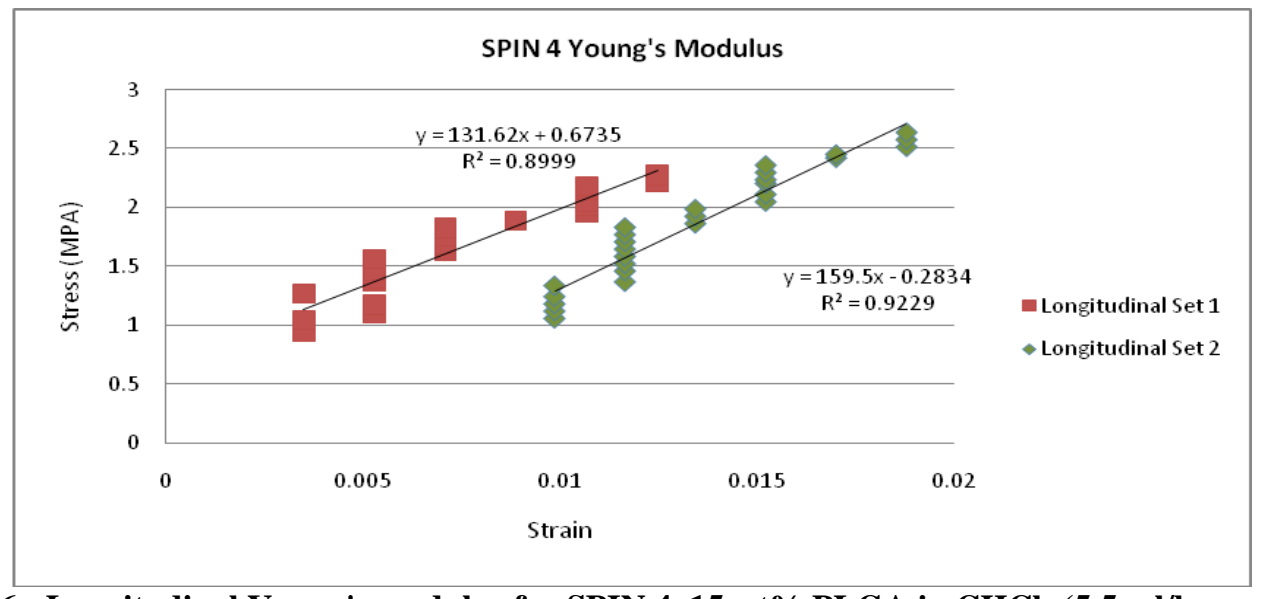

Figure 66 - Longitudinal Young's modulus for SPIN 4, 15 wt\% PLGA in $\mathrm{CHCl}_{3}(5.5 \mathrm{ml} / \mathrm{hr}$; unknown).

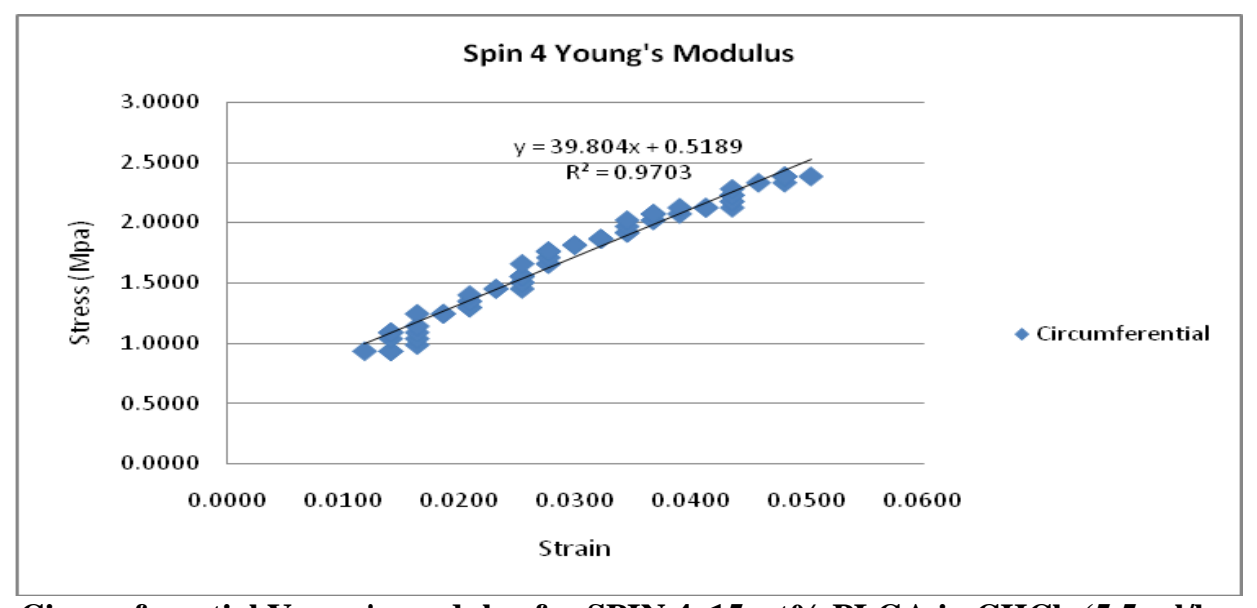

Figure 67 - Circumferential Young's modulus for SPIN 4, 15 wt \% PLGA in $\mathrm{CHCl}_{3}(5.5 \mathrm{ml} / \mathrm{hr}$; unknown). 


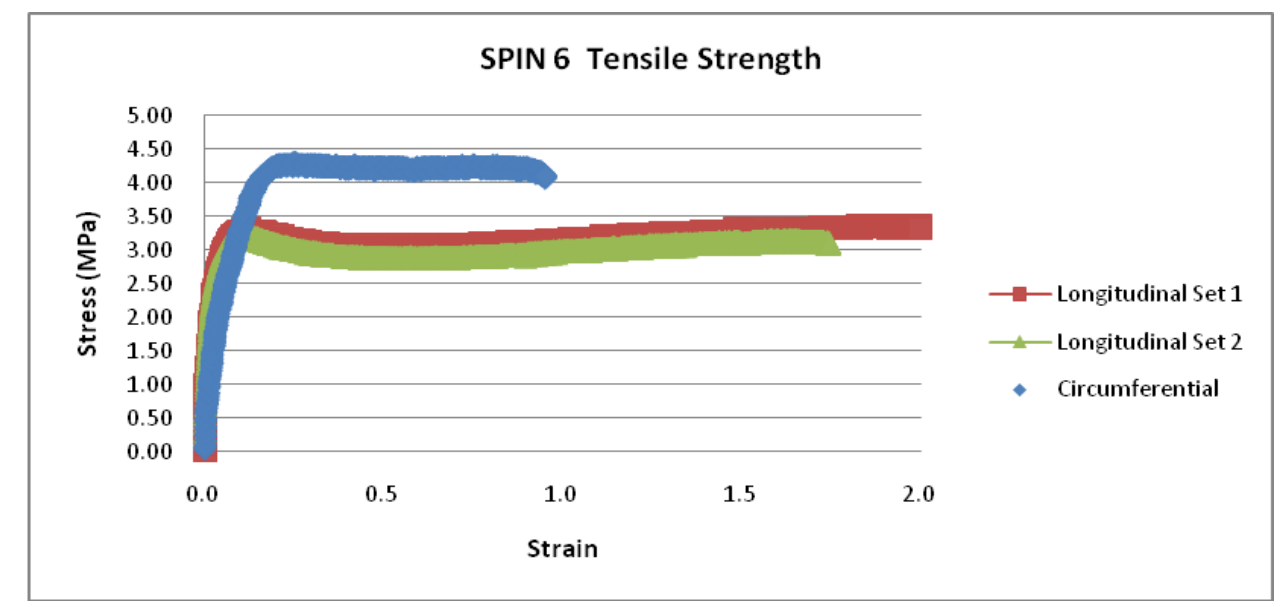

Figure 68 - Tensile strength for SPIN 6, $15 \mathrm{wt} \%$ PLGA in $\mathrm{CHCl}_{3}(5.5 \mathrm{ml} / \mathrm{hr} ; 12,000 \mathrm{~V})$.

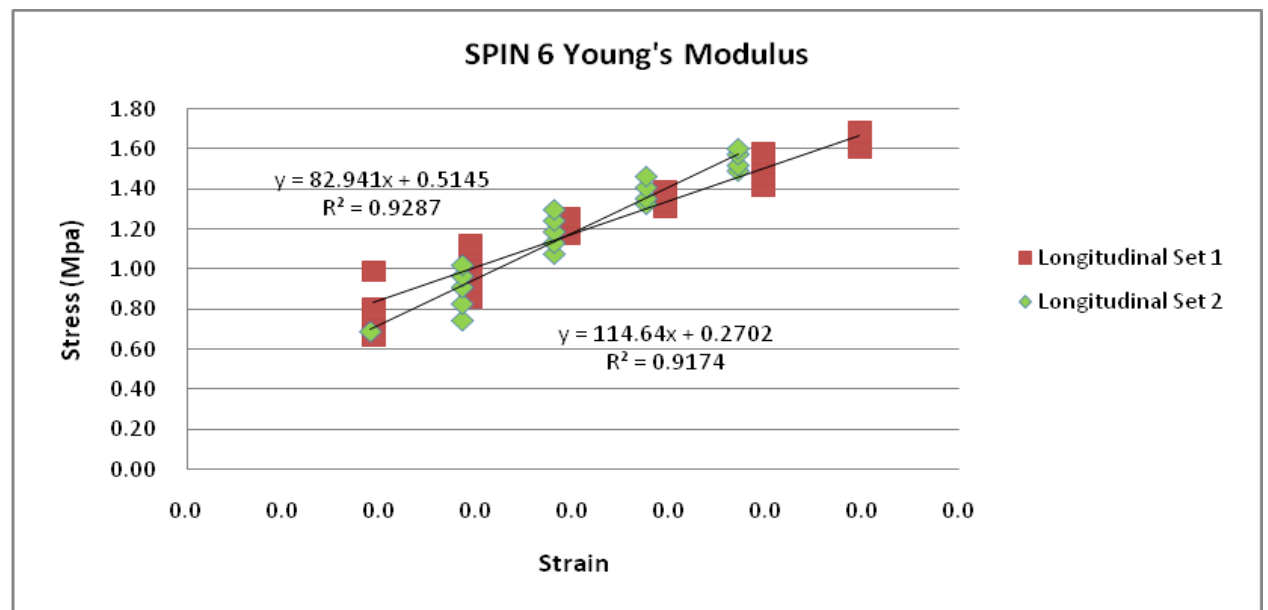

Figure 69 - Longitudinal Young's modulus, SPIN 6, $15 \mathrm{wt} \%$ PLGA in $\mathrm{CHCl}_{3}(5.5 \mathrm{ml} / \mathrm{hr} ; 12,000 \mathrm{~V})$.

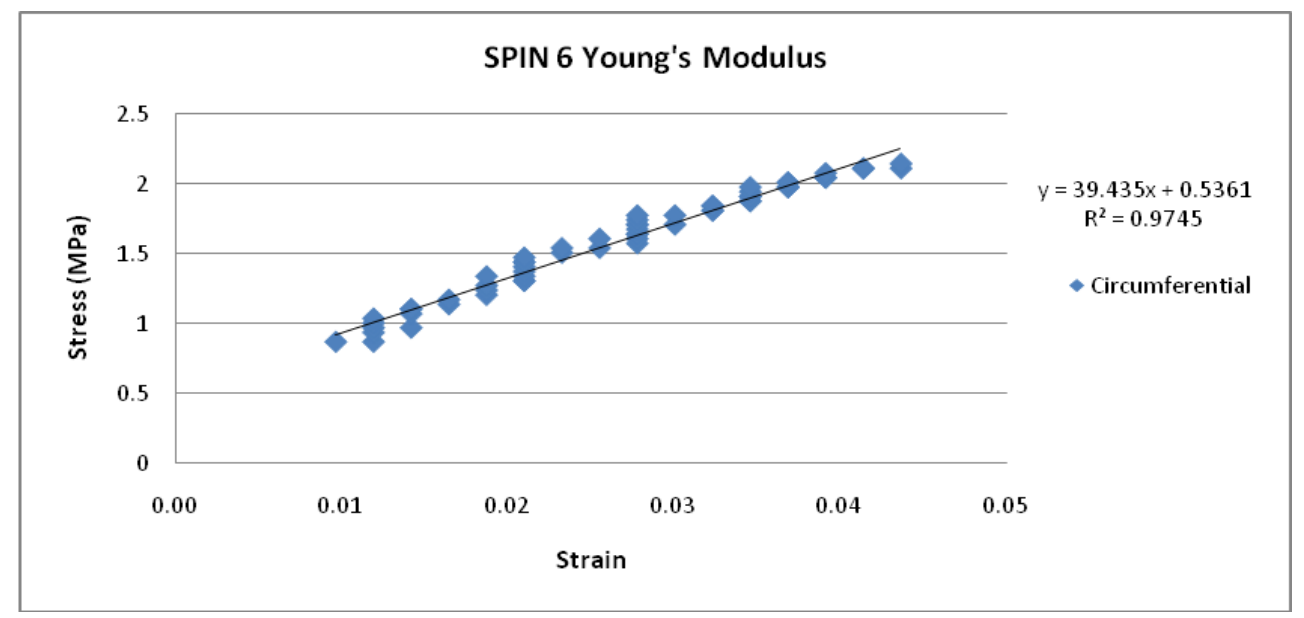

Figure 70 - Circumferential Young's modulus, SPIN 6, 15 wt \% PLGA in $\mathrm{CHCl}_{3}(5.5 \mathrm{ml} / \mathrm{hr} ; 12,000 \mathrm{~V})$. 


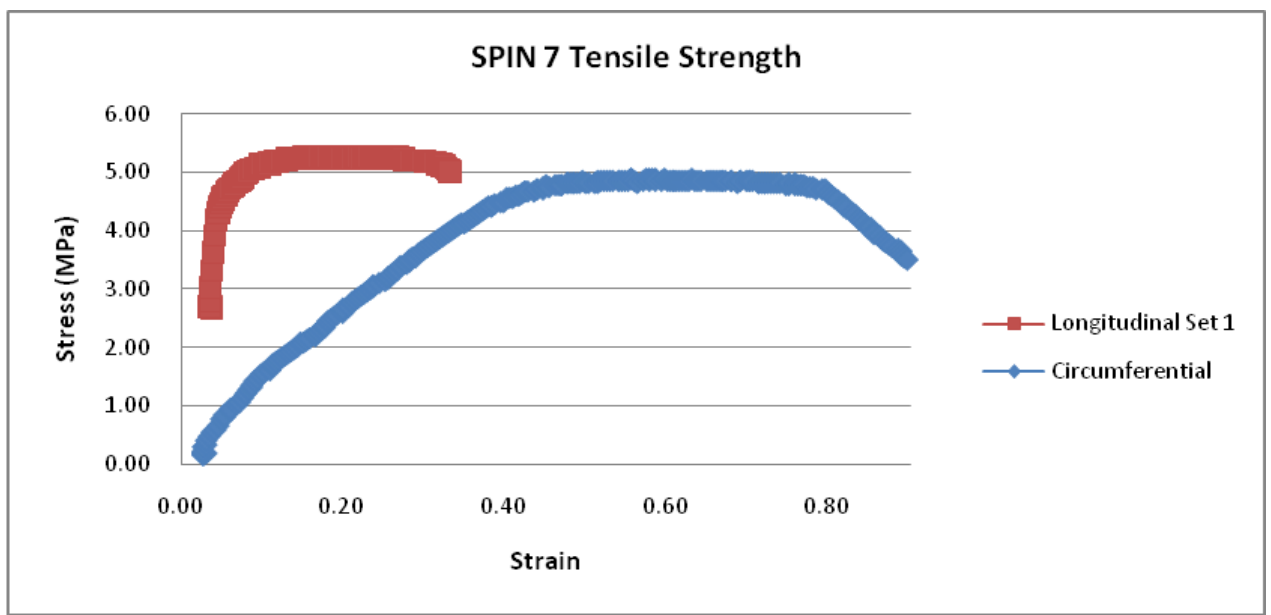

Figure 71 - Tensile strength for SPIN 7, 15 wt\% PLGA in $\mathrm{CHCl}_{3}(3.0 \mathrm{ml} / \mathrm{hr} ; 12,000 \mathrm{~V})$.

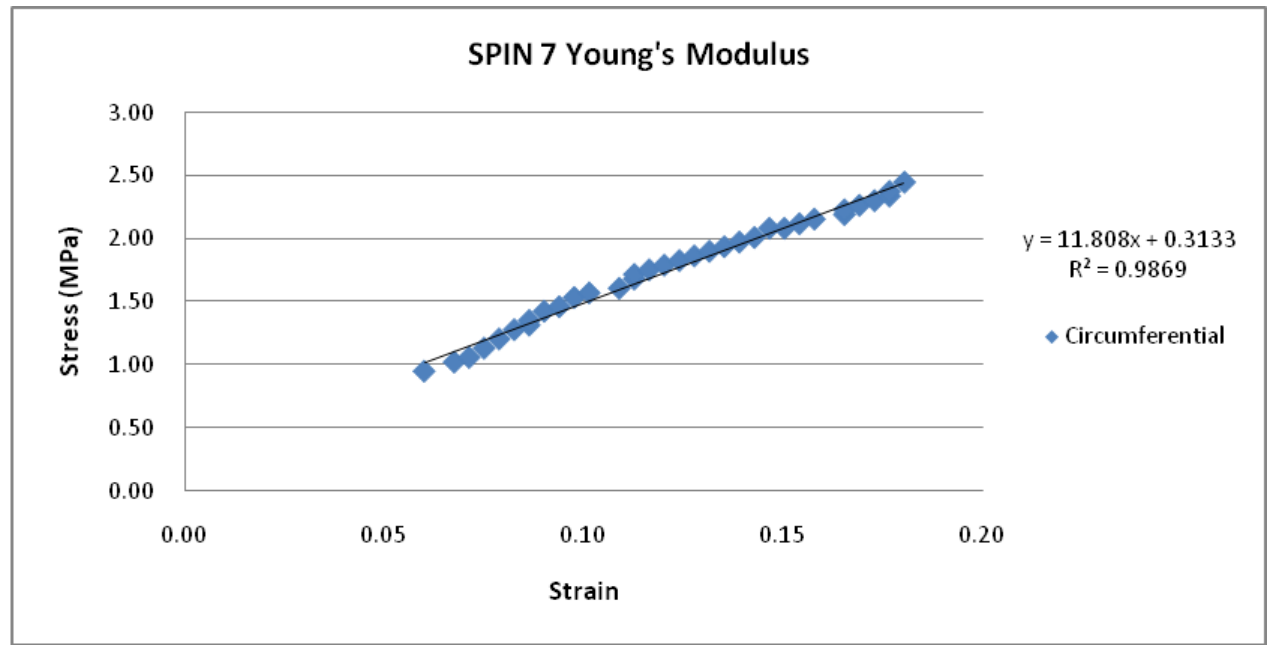

Figure 72 - Circumferential Young's modulus, SPIN 7, $15 \mathrm{wt} \%$ PLGA in $\mathrm{CHCl}_{3}(3.0 \mathrm{ml} / \mathrm{hr} ; 12,000 \mathrm{~V})$. 


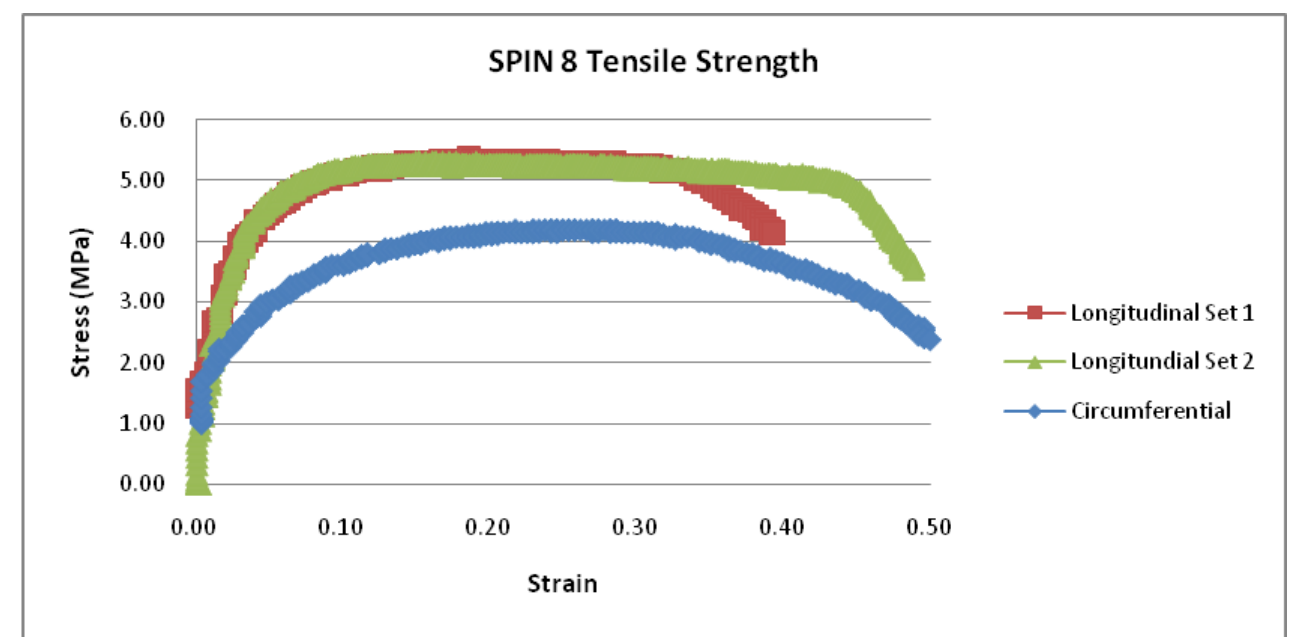

Figure 73 - Tensile strength for SPIN 8, 15 wt\% PLGA in $\mathrm{CHCl}_{3}(3.0 \mathrm{ml} / \mathrm{hr} ; 15,600 \mathrm{~V})$.

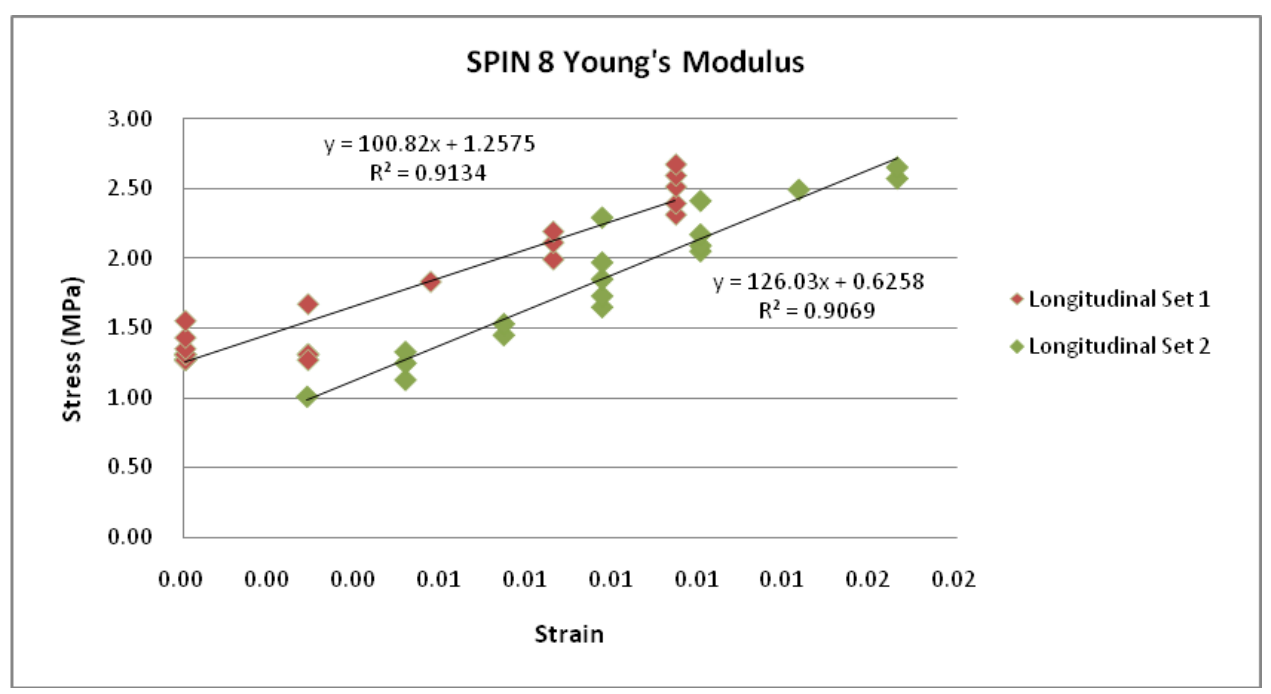

Figure 74 - Longitudinal Young's modulus, SPIN 8, 15 wt\% PLGA in $\mathrm{CHCl}_{3}(3.0 \mathrm{ml} / \mathrm{hr} ; 15,600 \mathrm{~V})$.

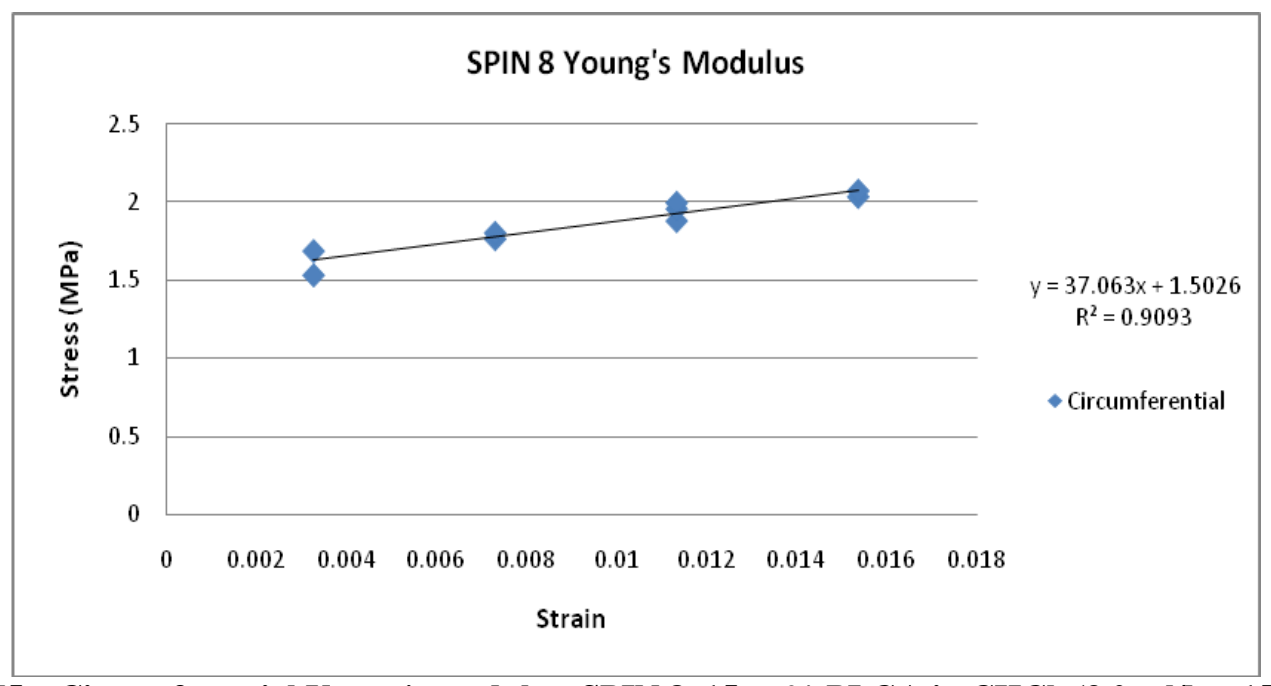

Figure 75 - Circumferential Young's modulus, SPIN 8, $15 \mathrm{wt} \%$ PLGA in $\mathrm{CHCl}_{3}(3.0 \mathrm{ml} / \mathrm{hr} ; 15,600 \mathrm{~V})$. 


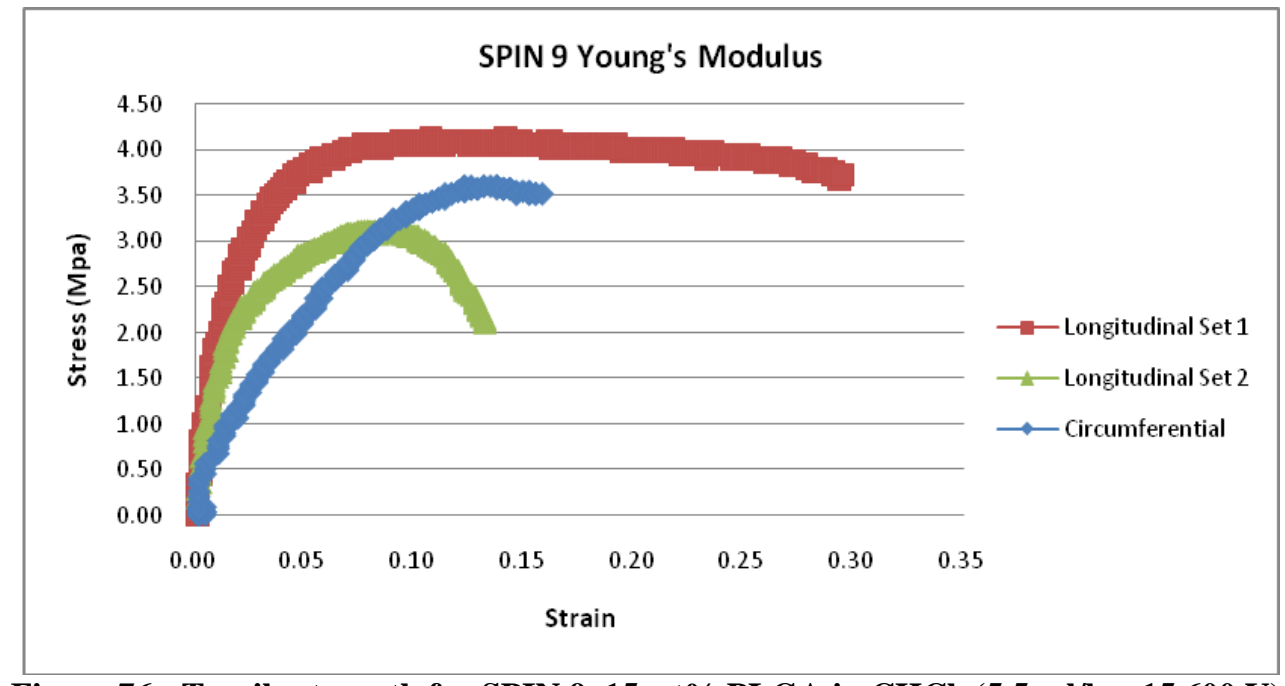

Figure 76 - Tensile strength for SPIN 9, 15 wt\% PLGA in $\mathrm{CHCl}_{3}(5.5 \mathrm{ml} / \mathrm{hr} ; 15,600 \mathrm{~V})$.

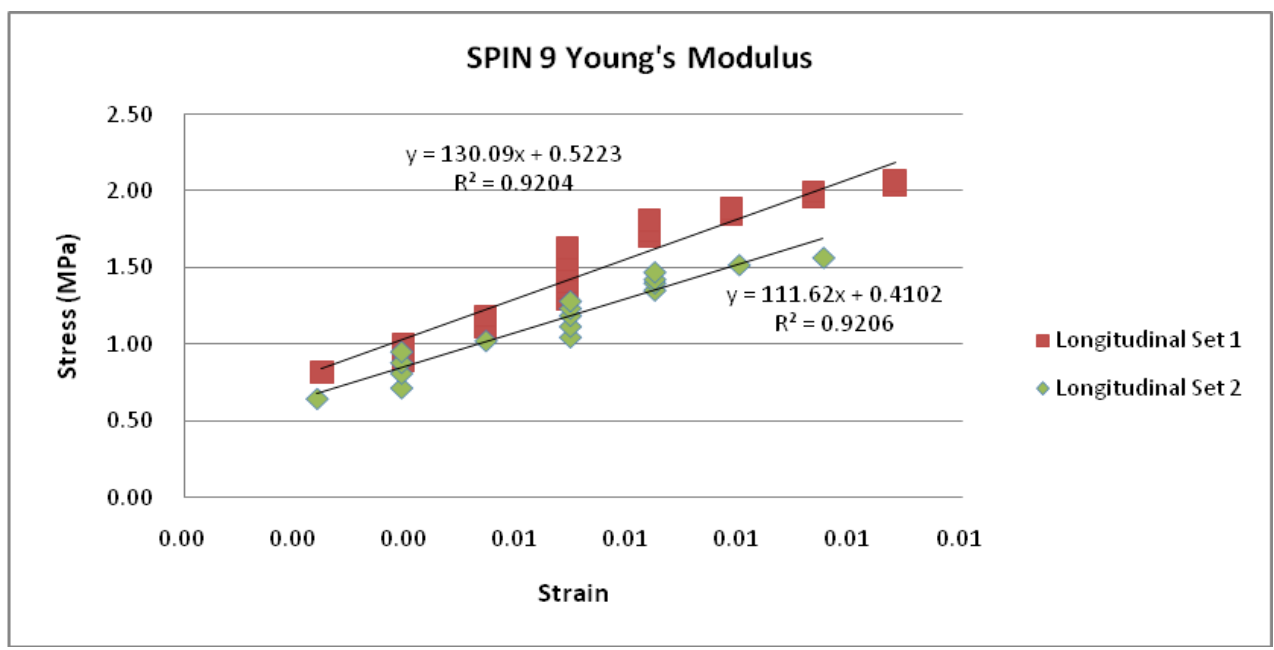

Figure 77 - Longitudinal Young's modulus for SPIN 9, $15 \mathrm{wt} \%$ PLGA in $\mathrm{CHCl}_{3}(5.5 \mathrm{ml} / \mathrm{hr}$; 15,600 V).

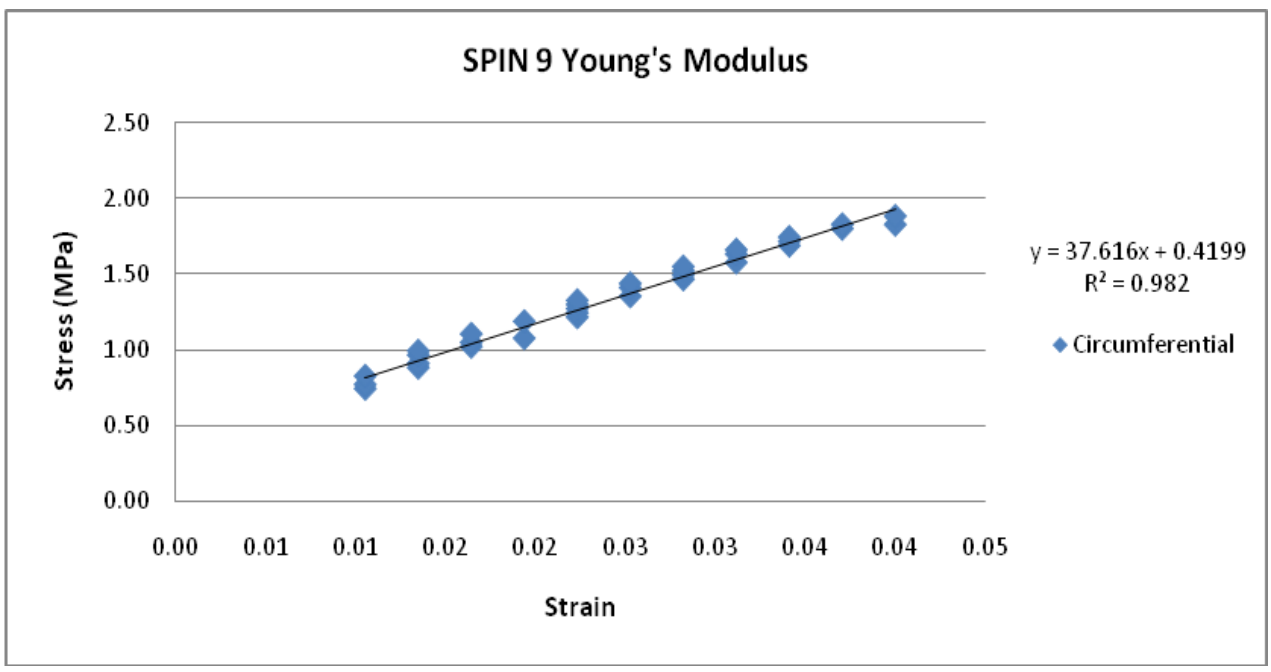

Figure 78 - Circumferential Young's modulus for SPIN 9, $15 \mathrm{wt} \%$ PLGA in $\mathrm{CHCl}_{3}(5.5 \mathrm{ml} / \mathrm{hr}$; 15,600 V). 


\section{Appendix H: Standard BVM Protocol}

\author{
Date \\ Study Information \\ Study Title: \\ Purpose: \\ Material: \\ Diameter \\ Length: \\ Cell Type: \\ Prep 1 week prior \\ 1. Gas sterilize biochambers and 2-port \\ reservoirs \\ 2. Determine target number of cells and \\ passage schedule, then thaw cells \\ 3. Cut grafts, mount on fittings, and suture \\ 4. Autoclave grafts, flasks, and forceps
}

Prep the day before

5. Make media:

a. Bioreactor Media (Human Complete w/o ECGS w/ antibiotics)

b. Conditioning Media (1:6 solution of FBS:M199 + antibiotics)

6. Denucleate grafts (using filtered $\mathrm{EtOH}$ )

a. $15 \min 70 \% \mathrm{EtOH}$

b. $15 \mathrm{~min} 100 \% \mathrm{EtOH}$

c. Leave in degassed Conditioning Media in incubator overnight

Set-up day: BVM conditioning

7. Warm up media (Bioreactor Media and Conditioning Media)

8. Insert sterile grafts into biochambers "**requires non-sterile partner**

9. Using $60 \mathrm{~mL}$ syringe, flush lumen with Conditioning Media to prime graft

a. Clamp lumen and continue to prime graft and chamber until chamber is full

b. Repeat for all vessels

10. Place small WM pump in hood

11. Prime 2-port reservoirs with Conditioning Media

12. Attach primed biochamber to 2-port reservoir and condition graft for $10 \mathrm{~min}$

a. Flow through lumen first to remove air, then clamp lumen and condition transmurally on $90 \mathrm{rpm}$ setting

13. Leave primed biochambers in large incubator until ready for sodding step

14. Prime 2-port reservoirs with Bioreactor Media

a. Prepare one for each vessel

b. Be sure that drip is visible and outlet is submerged

c. Clamp tubing and leave in big incubator

Set-up day: BVM sodding

15. Take corresponding number of primed biochambers and reservoirs to hood
Initials

16. Attach outlet of reservoir to inlet stopcock of biochamber

a. Leave reservoir inlet unattached and biochamber outlet facing trough

17. Record BVM numbers:

18. Harvest cells

a. Apply Trypsin, deactivate with media

b. Take $100 u$ from total $\mathrm{mL}$ cells Counts:

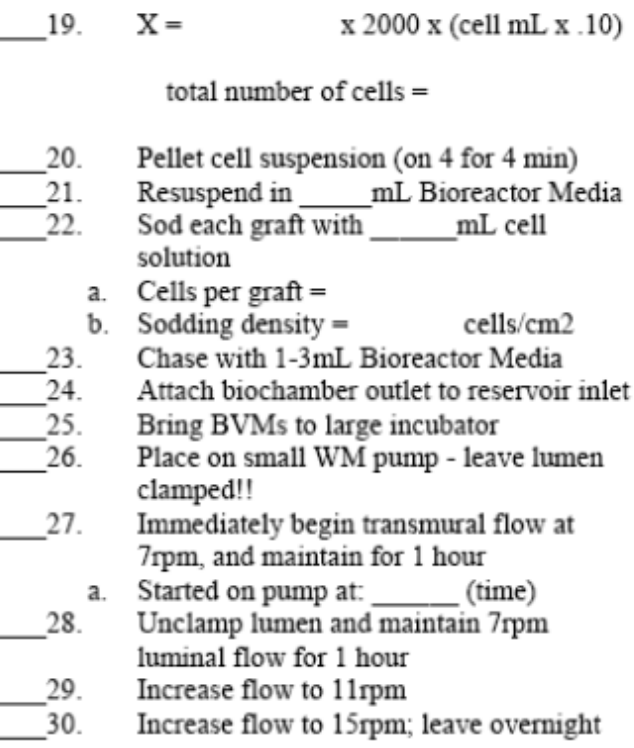

The next day and beyond

31. Increase flow by $10-15 \mathrm{rpm}$ at a time to reach $90 \mathrm{rpm}$ by the end of the day

32. BVM maintenance: replace media reservoirs every $3^{\text {rd }}$ day

a. Check $\mathrm{CO}$ !

BVM Usage

Stented:

OCT Imaged:

BVM Analysis

Other:

BBI:

SEM:

Histology/Stains: 


\title{
Appendix I: Copyright Permission Requests
}

\author{
Dear Tiffany:
}

On behalf of the National Heart, Lung, and Blood Institute (NHLBI) Health Information Center, we are responding to your request for information on the NHLBI copyright permission policy.

The information contained in materials published by the NHLBI is in the public domain. No further permission is required to reproduce or reprint the information in whole or in part.

However, organizations that reproduce NHLBI publications should cite the National Heart, Lung, and Blood Institute as a part of the National Institutes of Health and the U.S. Department of Health and Human Services as the source. This applies to printed publications as well as documents from the NHLBI Web site. Organizations may add their own logo or name. We further ask that no changes be made in the content of the material, and that the material as well as any NHLBI Internet links should not be used in any direct or indirect product endorsement or advertising.

Sincerely,

NHLBI Health Information Center

PO Box 30105

Bethesda, MD 20824

Phone: 301-592-8573

Fax: 301-592-8563

E-mail:nhlbiinfo@nhlbi.nih.gov

Web site: http://www.nhlbi.nih.gov 
Sorry for the delay--yes you have permission.

Regards,

Rick

Richard Gersony MFA CMI

President, Medical Illustrator

www.medmovie.com

\section{medmovie}

167 West Main Street

Suite 1210

Lexington, KY 40507

Fax: $\quad 859.225 .6401$

Phone: 859.225.6400 